\title{
HAND AND FOOT MUSCULATURE OF ANURA: STRUCTURE, HOMOLOGY, TERMINOLOGY, AND SYNAPOMORPHIES FOR MAJOR CLADES
}

BORIS L. BLOTTO, MARTÍN O. PEREYRA, TARAN GRANT, AND JULIÁN FAIVOVICH

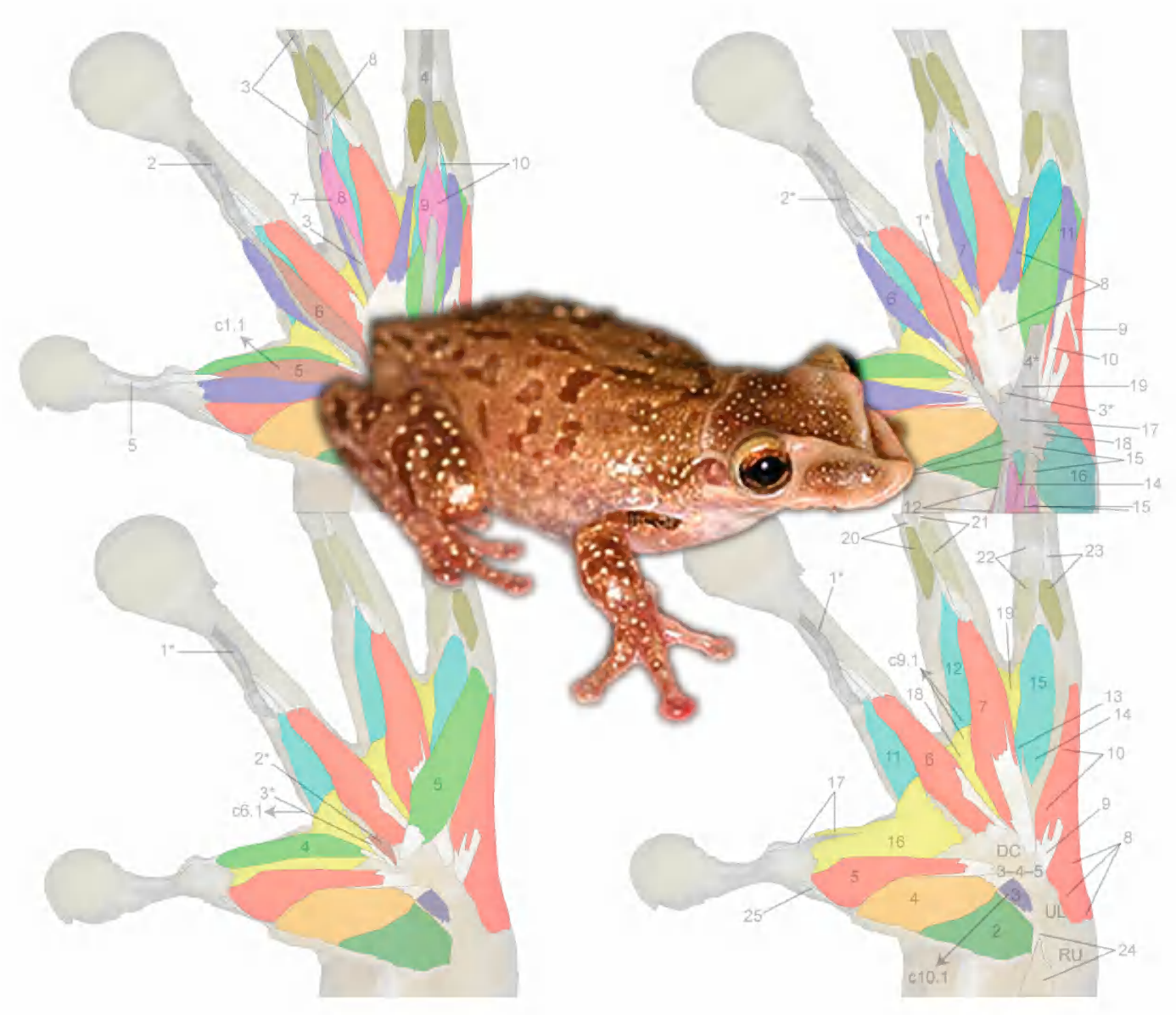

BULLETIN OF THE AMERICAN MUSEUM OF NATURAL HISTORY 


\title{
HAND AND FOOT MUSCULATURE OF ANURA: STRUCTURE, HOMOLOGY, TERMINOLOGY, AND SYNAPOMORPHIES FOR MAJOR CLADES
}

\author{
BORIS L. BLOTTO
}

Departamento de Zoologia, Instituto de Biociências, Universidade de São Paulo, São Paulo, Brazil; División Herpetología, Museo Argentino de Ciencias Naturales "Bernardino Rivadavia"-CONICET, Buenos Aires, Argentina

\section{MARTÍN O. PEREYRA}

División Herpetología, Museo Argentino de Ciencias Naturales "Bernardino Rivadavia"-CONICET, Buenos Aires, Argentina; Laboratorio de Genética Evolutiva "Claudio J. Bidau," Instituto de Biología Subtropical-CONICET, Facultad de Ciencias Exactas Químicas y Naturales, Universidad Nacional de Misiones, Posadas, Misiones, Argentina

\section{TARAN GRANT}

Departamento de Zoologia, Instituto de Biociências, Universidade de São Paulo, São Paulo, Brazil; Coleção de Anfíbios, Museu de Zoologia, Universidade de São Paulo, São Paulo, Brazil; Research Associate, Herpetology, Division of Vertebrate Zoology, American Museum of Natural History

\section{JULIÁN FAIVOVICH}

División Herpetología, Museo Argentino de Ciencias Naturales "Bernardino Rivadavia"-CONICET, Buenos Aires, Argentina; Departamento de Biodiversidad y Biología Experimental, Facultad de Ciencias Exactas y Naturales, Universidad de Buenos Aires, Buenos Aires, Argentina; Research Associate, Herpetology, Division of Vertebrate Zoology, American Museum of Natural History

BULLETIN OF THE AMERICAN MUSEUM OF NATURAL HISTORY

Number 443, 155 pp., 14 plates, 1 table

Issued November 6, 2020 


\section{CONTENTS}

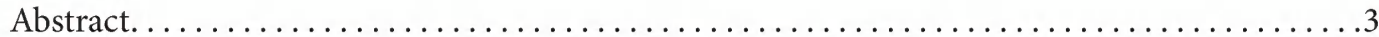

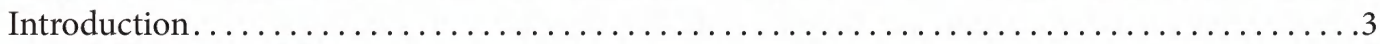

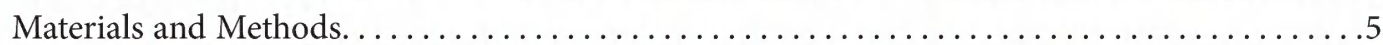

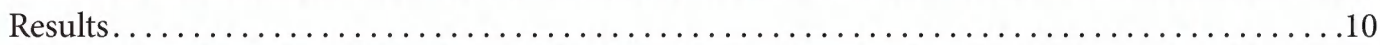

Description of the Hand and Foot Musculature of Triprion petasatus $\ldots \ldots \ldots \ldots \ldots \ldots$

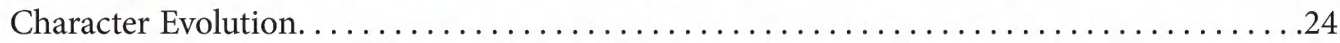

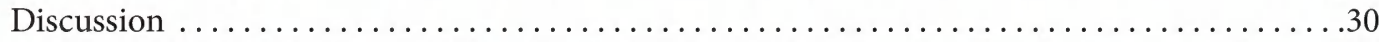

Systematic Implications of the Inferred Synapomorphies. . . . . . . . . . . . . . 30

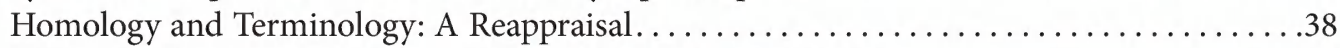

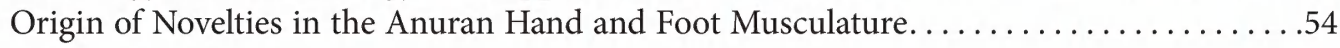

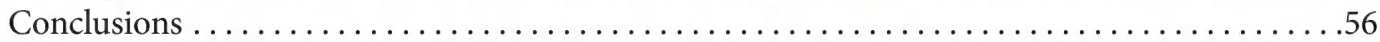

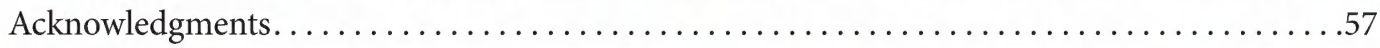

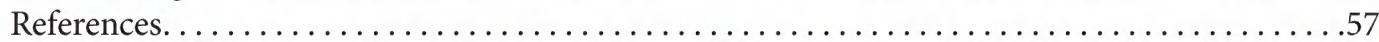

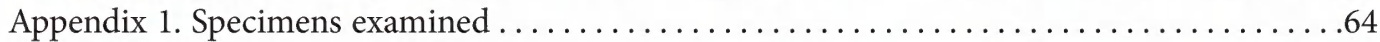

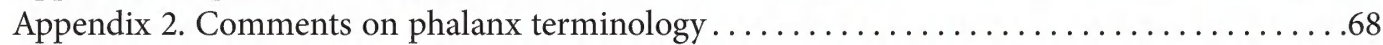

Appendix 3. Anuran hand and foot musculature: synonym list................... 70

Appendix 4. Data matrix . . . . . . . . . . . . . . . . . . . . . . . . . 112

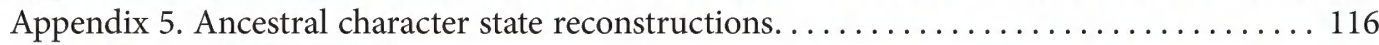

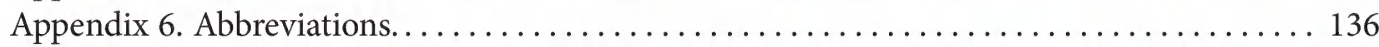

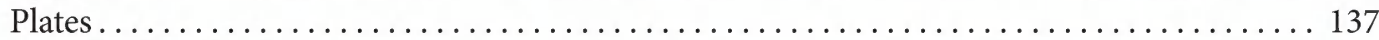




\begin{abstract}
Although studies of anuran hand and foot musculature began in the first half of the 19th century, all studies to date have been taxonomically or anatomically restricted in scope, and none has considered the diversity of autopodial myology in Anura as a whole. As a model for future comparisons, we thoroughly describe the hand and foot musculature of an arboreal species (the hylid Triprion petasatus), define the layers in which these muscles are arranged, and attribute presumed functions. On the basis of our myological analysis of 155 species representing 46 of the 54 currently recognized families and main clades of anurans, we describe 20 characters related to hand and foot muscles. Optimization of these characters on the most recent and inclusive phylogenetic hypothesis of Anura results in synapomorphies for several major clades (Bombinatoridae, Alytidae, Xenoanura + Acosmanura, Xenoanura, Pipidae, Acosmanura, Anomocoela, Scaphiopodidae, Pelodytidae + Pelobatidae + Megophryidae, Megophryidae, Neobatrachia, Heleophrynidae, Sooglossidae, Laurentobatrachia, Calyptocephalellidae, Myobatrachoidea, and Nobleobatrachia), including new, nonhomoplastic synapomorphies for clades previously supported only by molecular evidence and a few conflicting phenotypic characters (e.g., Acosmanura, Anomocoela, Neobatrachia). Additionally, we (1) address controversies regarding the homology of anuran and caudate muscles in the context of putative synapomorphies for Ascaphidae + Leiopelmatidae and its sister clade Lalagobatrachia; (2) evaluate a recently proposed terminology for anuran hand and foot musculature; (3) discuss the identities of several hand and foot muscles with problematic homologies; (4) establish a unified terminology for anuran hand and foot muscles, including a list of synonyms for all names employed in the literature; and (5) propose hypotheses for the origin of several myological novelties (neomorphs).
\end{abstract}

\section{INTRODUCTION}

Over the last 15 years, several studies have contributed significantly to the knowledge of phylogenetic relationships of major groups of Anura. Most of these studies were based exclusively on DNA sequences (e.g., Darst and Cannatella, 2004; Roelants et al., 2007; Heinicke et al., 2009; Wiens et al., 2010; San Mauro, 2010; Pyron and Wiens, 2011; Fouquet et al., 2013; Barej et al., 2014; Padial et al., 2014; Pyron, 2014; Brown et al., 2015; Frazão et al., 2015; Chan and Brown, 2017; Feng et al., 2017; Mahony et al., 2017; Chan et al., 2018; Jetz and Pyron, 2018; Streicher et al., 2018; Tu et al., 2018), while a few of them also included phenotypic datasets (Faivovich et al., 2005; Scott, 2005; Wiens et al., 2005; Frost et al., 2006; Grant et al., 2006, 2017) and even fewer focused exclusively on phenotypic characters-Haas (2003), Púgener et al. (2003), and Burton (2004) the major examples. Whereas Haas (2003) and Púgener et al. (2003) employed primarily larval characters scored for exemplars of major anuran lineages, Burton (2004) focused on the foot musculature of "Hylidae" (including Hemiphractidae). Both the larval and foot musculature studies proposed several synapomorphies for a number of groups, and their data sets were included in total evidence analyses by Frost et al. (2006) and Faivovich et al. (2005), respectively. However, the dearth of comparable data sets prevents these character systems from being used to test hypotheses across Anura.

Phenotypic synapomorphies are unknown for several major clades of anurans due to the lack of comprehensive studies for most character systems. This scenario underscores the need to explore multiple phenotypic sources of evidence to bear on phylogenetic hypotheses. The detailed study of phenotypic character systems provides homology hypotheses that are instrumental for the study of the morphological evolution of a clade (e.g., O'Leary et al., 2013). Furthermore, it allows the establishment of a bridge between pattern and process, enabling the exploration of evolutionary mechanisms and formulation of 
testable functional hypotheses (e.g., Wake et al., 2011). Ultimately, the inclusion of phenotypic data in total evidence phylogenetic analyses has been shown to have a disproportionately large effect, influencing tree topology and clade support estimations, and, therefore, taxonomic decisions (e.g., Nylander et al., 2004; de Sá et al., 2014; Mirande, 2017; Sánchez-Pacheco et al., 2018; Cabra-García and Hormiga, 2019).

Hand and foot muscles are an underexplored source of phenotypic data. The seminal works of Dugés (1834), Ecker (1864), Perrin (1892), Gaupp (1896), and Ribbing (1907, 1909, 1911) described in detail the musculature of a few anuran species, but few subsequent additional studies dealt with this character system until the end of the 1990s, and those that did mostly focused on single species (e.g., Davies and Burton, 1982; Burton, 1983) or a limited number of characters (e.g., Liem, 1970; Burton, 1986). The sole exception in that period is Dunlap (1960), who studied a diverse sample of species and characters of hind-limb musculature.

Beginning in the latter half of the 1990s, there has been a haphazard and idiosyncratic increase in knowledge of anuran hand and foot musculature without attempting to standardize and synthesize information across the major anuran clades. Burton (1996, 1998a) studied the hand musculature of Pelodryadinae and several families formerly included in "Leptodactylidae," respectively. Additionally, Burton (1998b) explored a few hand musculature characters using a dense sample of anurans, and Burton (1998c) studied the muscles associated with climbing. Burton (2001) also studied the foot musculature of the Australian "Myobatrachidae" (including Limnodynastidae). Several studies focused on Hylidae, and characters from musculature were included in quantitative phylogenetic analyses: Faivovich (2002) and Araujo-Vieira et al. (2019) included some characters from the hand and foot musculature of Scinax and Sphaenorhynchus and related groups, respectively; Burton (2004) performed an extensive survey of the foot musculature of "Hylidae" (including Hemiphractidae), which Faivovich et al. (2005) combined with DNA sequences in a total evidence analysis. Manzano et al. (2008) studied characters related to specializations for grasping in the forelimb of two hylids. Salgar et al. (2009) reported on the foot musculature of a species of Pristimantis (Craugastoridae), whereas Hoyos et al. (2014) defined characters of foot musculature in a quantitative phylogenetic analysis of the Pristimantis unistrigatus species group, and Hoyos and Salgar (2016) focused on variation within Dendropsophus luddeckei (as D. labialis; Hylidae). Diogo and Ziermann (2014) studied the development of the fore- and hind-limb muscles using Eleutherodactylus coqui (Eleutherodactylidae) as a model. Blotto et al. (2017) described the foot musculature of Odontophrynidae, proposed synapomorphies, and discussed putative adaptations for burrowing. Finally, some studies focused on a few muscles of the hand and/or foot within a morpho-functional framework (e.g., Hanna and Barnes, 1991; Dos Santos et al., 2017; Abdala et al., 2018; Fratani et al., 2018, 2020; Oliveira-Lagôa et al., 2019; Soliz et al., 2020).

Given the lack of comparable information on the hand and foot musculature across the major clades of Anura, we examined variation in this musculature across a broad sample of Anura in an effort to (1) identify the musculature layers in which the hand and foot muscles are arranged and attribute presumed functions; (2) delimit putatively homologous character states; (3) optimize those characters on a recent hypothesis of anuran relationships to study character evolution; (4) when relevant, critically assess previously proposed phenotypic synapomorphies for major clades in the context of our findings and recent phylogenetic hypotheses; (5) identify outstanding problems in the homology and terminology of anuran muscles and provide a unified terminology; and (6) propose hypotheses for the origin of novel muscles and slips. 


\section{MATERIALS AND METHODS}

\section{Taxon Sampling}

We sampled all major anuran clades supported in recent phylogenetic hypotheses (e.g., Frost et al., 2006; Pyron and Wiens, 2011; Pyron, 2014; Jetz and Pyron, 2018) and most families of Anura. For Costata, Pipidae, Anomocoela, Heleophrynidae, and Sooglossidae we sampled more than one species per genus when available (i.e., Bombina, Discoglossus, Heleophryne, Hymenochirus, Megophrys, Pelobates, Pipa, Scaphiopus, Sooglossus, Spea, and Xenopus). This is justified on the basis that several synapomorphies proposed are for deep nodes, and redundant taxon sampling (e.g., more than one species of Bombina) serves as an additional test of the proposed synapomorphies. Heleophrynidae and Sooglossoidea (Nasikabatrachidae + Sooglossidae) were recovered in alternative phylogenetic positions in recent phylogenetic analyses (Biju and Bossuyt, 2003; Frost et al., 2006; Roelants et al., 2007; Pyron and Wiens, 2011; Kurabayashi and Sumida, 2013; Zhang et al., 2013; Pyron, 2014; Jetz and Pyron, 2018) but always as the sister taxon of Ranoides, Notogaeanura, or Neobatrachia. On this basis, we sampled three heleophrynids (the monotypic Hadromophryne and two species of Heleophryne) and the two species of Sooglossus. We were unable to sample 8 of the 54 anuran families: Conrauidae, Mantellidae, Micrixalidae, Nasikabatrachidae, Nyctibatrachidae, Odontobatrachidae, Petropedetidae, and Ranixalidae. With the exception of Nasikabatrachidae, the remaining families are members of the well-supported clade Natatanura, which is nested in Ranoides. We were able to sample some families of Natatanura (Ceratobatrachidae, Dicroglossidae, Phrynobatrachidae, Ptychadenidae, Pyxicephalidae, Ranidae, and Rhacophoridae), and the lack of the remaining families of the clade is expected to have no or little impact in the ancestral character-state reconstructions (which are mainly focused on major clades).
We employed the phylogenetic hypothesis of Jetz and Pyron (2018) for ancestral state reconstruction (see below), with the addition of Atelopus pastuso (Bufonidae), Leptobrachium lumadorum, and Megophrys edwardinae (both Megophryidae). We placed $A$. pastuso in the topology of Jetz and Pyron (2018) following the evidence presented by Coloma et al. (2010), and the megophryids on the basis of evidence from Brown et al. (2010) and Chen et al. (2017).

Finally, in order to test, interpret, compare, and discuss some relevant reports from the literature we performed partial dissections of additional species not scored in our analysis. These species are: Dendropsophus luddeckei (Hylidae; for comparison with Hoyos and Salgar, 2016), Osteopilus septentrionalis (Hylidae; for comparison with Hanna and Barnes, 1991), Pipa arrabali (Pipidae; for comparison with Burton, 1998b), and Pipa pipa (Pipidae; for comparison with Ribbing, 1911). Specimens examined and locality data are listed in appendix 1. Collection codes used throughout the paper are those of Sabaj (2016).

\section{Targeted Muscles}

The targeted muscles of the hand are restricted to those that originate or insert on the prepollex, metacarpals, and/or phalanges, with the exception of the m. contrahentis caput longus distalis, which was studied here despite its origin on the ulnare and insertion on the distal carpals. The targeted muscles of the leg and foot are those with attachments to the fibulare, tibiale, distal tarsals, element Y, prehallux, metatarsals, and/or phalanges.

\section{Terminology}

The anuran taxonomy follows Frost (2019), except for Hylidae, which follows Faivovich et al. (2018). Terminology for the carpus and tarsus follows Fabrezi $(1992,1993)$ and Fabrezi and Alberch (1996). The phalanges are referred to as the "basal phalanx" and "distal phalanx" for the proximal and distal phalanges in digits with two phalanges, respectively. For phalanges between 
the "basal" and "distal" phalanges in digits with three phalanges, we employ the term "second phalanx"; for digits with four phalanges, we employ "second phalanx" for the more proximal phalanx and "third phalanx" for more distal phalanx. Although there are anuran species with digital reduction, all descriptions done across this study are for species with the complete phalangeal formula, which is 2-2-3-3 (i.e., two phalanges in digits II-III and three in digits IV-V) for the hand and 2-2-3-4-3 for the foot. This scheme is compared with the other commonly employed terminology in anurans in appendix 2 .

With respect to the hand and foot musculature, most anuran studies (e.g., Dunlap, 1960; Burton, 1996, 1998a, 1998b, 1998c, 2001, 2004) are largely based on the terminology of Gaupp (1896), with a few modifications introduced by Dunlap (1960), Burton (1996, 2004), and Faivovich (2002); we refer to this as the "classical terminology." Over the past decade, several studies (Abdala and Diogo, 2010; Diogo and Abdala, 2010; Diogo and Molnar, 2014; Diogo and Ziermann, 2014; Diogo et al., 2018) have proposed modifications to the classical terminology in an effort to unify terminology across tetrapods. We attempted to use and adapt the terminology of Diogo and associates for Anura, addressing controversies and introducing modifications when necessary. However, although we agree that homology is a phylogenetic concept that is tested through both separate, independent tests and the test of character congruence (i.e., primary and secondary homology, respectively; Pinna, 1991) and that independently derived muscles are not the same historical entity, we disagree with Diogo and Abdala (2010: 19) and Diogo et al. (2018: 11) that these nonhomologous muscles must be given different names and coded as different characters, for the following reasons.

First, this approach would prove to be problematic in practice because these systems have multiple instances of homoplasy, resulting in the proliferation of names that are both unnecessary to comprehend character evolution and burdensome and potentially confusing for workers gath- ering anatomical evidence. Two muscles whose occurrence varies across Anura exemplify the problem (see Results for detailed accounts): the hand $\mathrm{m}$. lumbricalis longus digiti III (char. 2) and $\mathrm{m}$. flexor minimus indicis (char. 8). These muscles would require three and four different names, respectively, because they arise independently four and five times, respectively (see ancestral character state reconstructions in appendix 5).

Second, although this approach is consistent with the ontological individuality of each independently derived character state (see Grant and Kluge, 2004, 2009), renaming characters solely on the basis of their phylogenetic distribution without external evidence conceals contradictory evidence (i.e., character incongruence) and violates the logical distinction between premises and conclusions, leading to circular reasoning in future research cycles. That is, whereas reciprocal clarification or illumination (Hennig, 1966; Kluge, 1997) uses character incongruence heuristically to critically reexamine evidence and conduct additional independent tests of hypotheses of primary homology, the simple renaming of the characters eliminates such incongruence, preventing those characters from testing the hypothesis in the future. A similar argument was raised by Grant et al. (2006: 108) in relation to the proposal that the palatine of neobatrachians be renamed "neopalatine" (Trueb, 1993) on the basis of the phylogenetic position of Neobatrachia.

As such, the purpose of the terminology adopted in this paper is to indicate inferred primary homology, with the secondary nonhomology of character states whose homology is refuted by the congruence test indicated by separate statements, without renaming the muscles. In order to unify terminology across this study and avoid repeating references to alternative hand and foot names of other authors, we present a synonym list for all the terms employed in the literature (appendix 3). This synonym list indicates whether the name employed for each specific muscle is equivalent to the concept and name employed here. 
The hierarchic arrangement of muscles in this study is largely consistent with classic contributions (e.g., Dunlap, 1960; Burton, 1998a, 2004). Although this hierarchy is related to muscle homology, its main utility is in predefining the units referred to as "muscle group," "muscle," and "slip." For example, the group of the foot $\mathrm{mm}$. lumbricales digitorum breves is usually composed of seven muscles, one on digits I-III and two (one medial and one lateral) on digits IV and V. Following this example, the medial and lateral muscles of the $\mathrm{mm}$. lumbricales breves of digit $\mathrm{V}$ are termed "medial $\mathrm{m}$. lumbricalis brevis digiti V" and "lateral $\mathrm{m}$. lumbricalis brevis digiti V." The medial muscle is further divided into medial and lateral slips in some species, resulting in the terms "medial slip of the medial m. lumbricalis brevis digiti V" and "lateral slip of the medial $\mathrm{m}$. lumbricalis brevis digiti V." Thus, the hierarchy between muscle and slip avoids confusion.

The locational terms employed here are: (1) ventral, which is the side facing the ground and refers to the palmar (hand) or plantar (foot) side; (2) dorsal, which refers to the top (upper) side of the hand and foot facing the vertebral column; (3) proximal, in reference to attachments or portions topologically toward the base of the structure, near the body; (4) distal, in reference to attachments or portions topologically toward the tip of the structure, away from the body; (5) medial, preaxial, or radial/tibial side (these two last terms for the hand and foot, respectively); and (6) lateral, postaxial, or ulnar/fibular side (for the hand and foot, respectively). Points (5) and (6) are in reference to the sides with respect to the first and last digit, respectively.

We employ the term "intermediate" for designating a muscle or slip that is topologically positioned between medial and lateral muscles or slips (e.g., intermediate $\mathrm{m}$. dorsometacarpalis proximalis digiti III; intermediate slip of the $\mathrm{m}$. interphalangeus digiti IV). Our use of "intermediate" is equivalent to "central" sensu Burton (1998a: 63) for the hand $\mathrm{mm}$. interphalangei digitorum IV-V.

Finally, we are aware of several academic theses on hand and/or foot muscles (Andersen,
1978; Tyson, 1987; Wilkinson, 1993; Egan, 1995; Manzano, 1996; Silva, 1998). Although we consider them to be important contributions, they remain unpublished, and for this reason we do not discuss them in this study.

Dissection Procedures and Musculature Layers

Specimens were studied with the aid of a stereomicroscope. Dissections were performed sequentially to remove superficial layers and observe successively deeper muscles. Topical application of the iodine/potassium iodide solution of Bock and Shear (1972) was used to enhance contrast when necessary. The data matrix was built using the software Mesquite v3.03 (Maddison and Maddison, 2015).

The musculature of the hand and foot is arranged into layers (e.g., Burton, 1996, 1998a, 2004), which we used as a general guide for dissection, scoring, and imaging, with digital images of each layer captured before removal to both document our observations and enable revision and identification of novel characters and character states as species were added to the matrix. The arrangement of the musculature by layers mostly follows Burton, but two facts preclude an unequivocal arrangement. First, not all muscles can be objectively arranged into layers, such as some large abductors, flexors, and extensors of the forearm and leg. Second, in order to avoid removal of muscles that is not necessary for character coding or clearer interpretation of figures, we arranged some deep muscles into a fourth layer, even though some of them are more superficial than others. The layer arrangement of the hand and foot muscles followed in this study is shown in table 1 , along with the muscles unassigned to layer.

Following this framework, we removed the successive layers, defining four layers in each of the four systems studied here: palmar and dorsal surfaces of the hand and plantar and dorsal surfaces of the foot. Character scoring and image documentation were performed in parallel as each musculature layer was dissected and 
TABLE 1

Layer arrangement of the hand and foot musculature

\begin{tabular}{|c|c|c|c|c|}
\hline & Hand: ventral & Hand: dorsal & Foot: ventral & Foot: dorsal \\
\hline First layer & $\begin{array}{l}\text { Tendines superficiales, } \\
\text { flexor indicis superficialis } \\
\text { proprius, caput profundum } \\
\text { digiti III, lumbricales longi }\end{array}$ & Extensor digitorum & $\begin{array}{l}\text { Tendines superficiales, lum- } \\
\text { bricales longi, lumbricalis } \\
\text { longissimus digiti IV }\end{array}$ & $\begin{array}{l}\text { Extensor digitorum } \\
\text { longus }\end{array}$ \\
\hline Second layer & Lumbricales breves & $\begin{array}{l}\text { Abductor pollicis lon- } \\
\text { gus, extensores breves } \\
\text { superficiales }\end{array}$ & Lumbricales breves & $\begin{array}{l}\text { Extensores breves } \\
\text { superficiales }\end{array}$ \\
\hline Third layer & Contrahentes digitorum & $\begin{array}{l}\text { Extensores breves } \\
\text { medii }\end{array}$ & Contrahentes pedis & $\begin{array}{l}\text { Extensores breves } \\
\text { medii }\end{array}$ \\
\hline Fourth layer & $\begin{array}{l}\text { Pronator quadratus, contra- } \\
\text { hentis caput longus distalis, } \\
\text { adductor pollicis, flexores } \\
\text { breves profundi, abductor } \\
\text { digiti minimi, abductor } \\
\text { secundus digiti V, flexores } \\
\text { digitorum minimi, inter- } \\
\text { metacarpales, interpha- } \\
\text { langei }\end{array}$ & $\begin{array}{l}\text { Abductor indicis bre- } \\
\text { vis dorsalis, abductor } \\
\text { brevis digiti } \mathrm{V} \text {, dorso- } \\
\text { metacarpales }\end{array}$ & $\begin{array}{l}\text { Flexor hallucis accessorius, } \\
\text { flexores breves profundi, } \\
\text { abductor brevis plantaris } \\
\text { digiti V, flexores digitorum } \\
\text { minimi, adductor praehal- } \\
\text { lucis, intermetatarsales, } \\
\text { interphalangei }\end{array}$ & $\begin{array}{l}\text { Abductores breves } \\
\text { dorsales, abductor pro- } \\
\text { prius digiti IV, dorso- } \\
\text { metatarsales }\end{array}$ \\
\hline Unassigned & $\begin{array}{l}\text { Flexor digitorum commu- } \\
\text { nis, flexor accessorius, } \\
\text { flexor plate }\end{array}$ & $\begin{array}{l}\text { Extensor carpi radialis, } \\
\text { extensor carpi ulnaris }\end{array}$ & $\begin{array}{l}\text { Abductor praehallucis, } \\
\text { abductor brevis plantaris } \\
\text { hallucis, aponeurosis plan- } \\
\text { taris, flexor accessorius, } \\
\text { tibialis posterior, flexores } \\
\text { breves superficiales, contra- } \\
\text { hentium caput longum, } \\
\text { interosseus cruris }\end{array}$ & $\begin{array}{l}\text { Abductor digiti min- } \\
\text { imi, tarsalis anticus }\end{array}$ \\
\hline
\end{tabular}

removed. This predefined arrangement into layers facilitates interpretation and comparison of the different images/figures across species.

To facilitate among-group comparisons, we begin with a thorough description of the arboreal treefrog Triprion petasatus (Neobatrachia: Hylidae). The advantage of selecting an arboreal species as our model to illustrate the four layers of the four systems of the hand and foot musculature is that these species usually have additional groups of muscles (the $\mathrm{mm}$. dorsometacarpales distales and $\mathrm{mm}$. dorsometatarsales distales) and greater complexity resulting from the division of some muscles into multiple slips (e.g., the palmar m. flexor digitorum communis). The general description and figures of each layer of musculature of Triprion petasatus are used as a standard reference across the study (in analogy to a map), allowing a link between the particular layer exposed in each figure and the equivalent layer in T. petasatus. Each muscle group from each layer is edited in the different figures employing the same predefined color as in the figures of T. petasatus.

\section{Descriptive Approximations}

Muscles can be attached to phalanges, joints (between metacarpals/metatarsals and phalanges, or between phalanges), or both. We attempted to distinguish between these different points of attachment, since they are relevant not only for identifying character states but also for inferring function. However, in contrast to the 
more proximal phalanges and joints, the occurrence of an insertion on the distal interphalangeal joint (or interphalangeal joint in digits with two phalanges) and/or the distal phalanx is not usually discrete enough (especially in the $\mathrm{mm}$. dorsometacarpales/dorsometatarsales proximales/distales) to allow clear distinction between them. Distal phalanges are usually shorter than proximal ones, and the insertions on them are frequently a continuation of the insertion on the distal interphalangeal joint (e.g., see Langowski et al., 2018), making their distinction difficult or arbitrary. As such, we refer to these insertions as "(distal) interphalangeal joint/distal phalanx" throughout the text. Nevertheless, it must be clarified that there are cases in which the insertion is more dominant in one of these two regions; further studies quantifying these differences are required.

Similarly, the interpretation of the insertions can be difficult for muscles with long tendons of insertion that attach distally to one or more digital joint. For example, the mm. dorsometacarpales/ dorsometatarsales proximales that attach to the distal interphalangeal joint/distal phalanx also adhere partially to the connective tissue of additional proximal joints as their tendons pass through them. In these cases, we described the condition as an insertion on the distal interphalangeal joint/distal phalanx unless a discrete tendon of insertion leads to the more proximal joints.

\section{Image Editing}

Given the number of complex relationship among hand and foot muscles, we edited images in order to facilitate visualization, adjusting image opacity applying colors to shade the different elements (muscle groups, tendons, ligaments). After extensive experimentation we choose an intermediate opacity (40\%) for shading the muscles in order to make the edited images comparable when acquired from different specimens/species (differing in musculature color), acquisition hardware/ software, and pre- and postprocessing parameters. This percentage allows colors to be more or less equivalent across different images/species, thereby simplifying comparison and interpretation. Lower opacity precludes comparison due to the interaction of the selected color with the image background color, whereas higher opacity conceals some relevant aspects of the muscle (e.g., fiber orientation).

Ideally each muscle group should be labeled with a different, standardized color. However, in order to reduce the number of colors employed and enhance comparability across images, the following muscles were labeled with the same color.

M. flexor indicis superficialis proprius and $\mathrm{m}$. caput profundum digiti III (first layer of the palmar surface; pl. 2A): we found substantial evidence supporting the common origin of the $\mathrm{m}$. flexor indicis superficialis proprius and $\mathrm{m}$. caput profundum digiti III from a differentiation of the $\mathrm{m}$. flexor accessorius (see Discussion), leading us to apply the same color to both.

M. abductor secundus digiti $\mathrm{V}, \mathrm{m}$. abductor digiti minimi, and $\mathrm{mm}$. flexores breves profundi (fourth layer of the palmar surface; pl. 2D): although the former was considered part of the $\mathrm{mm}$. flexores breves profundi by some authors (see Discussion and appendix 3), there is no evidence relating the $\mathrm{m}$. abductor digiti minimi to the mm. flexores breves profundi. Nevertheless, on the basis of their similarity, they are shaded with the same color for the purpose of this study.

M. abductor indicis brevis dorsalis and $\mathrm{m}$. abductor brevis digiti V (fourth layer of the dorsal surface of the hand; pl. 3D): although both muscles are abductors located in the deepest layer, there is no evidence that they form a muscle group. Nevertheless, we use the same color on the basis of their equivalent function and position in the same layer.

M. flexor hallucis accessorius, $\mathrm{m}$. abductor brevis plantaris digiti $\mathrm{V}$, and $\mathrm{mm}$. flexores breves profundi (fourth layer of the plantar surface; pl. 4D): the first muscle was considered part of the $\mathrm{mm}$. flexores breves profundi group by some authors (see Discussion and appendix 3 ) and is morphologically similar to them, and the $\mathrm{m}$. abductor brevis plantaris digiti $\mathrm{V}$ is also 
morphologically and topologically very similar to the $\mathrm{mm}$. flexores breves profundi of digits II-IV. Additionally, the $\mathrm{m}$. abductor brevis plantaris digiti $\mathrm{V}$ was associated to the $\mathrm{mm}$. flexores breves profundi (Ribbing 1909, 1911; Diogo and Molnar, 2014; 1063; Diogo et al, 2018: 571). As such, we apply the same color to all of these muscles.

Tendinous/aponeurotic systems formed by the hand and foot tendines superficiales and tendons of insertion of the different muscles from which they arise: shading all of these elements in gray highlights their nonindependent connections and also avoids the need to arbitrarily delimit the different elements of this system, whose limits are not discrete. For example, the tendines superficiales of the hand are in fact the tendons of insertion of the $\mathrm{m}$. flexor digitorum communis and $\mathrm{m}$. flexor accessorius (in some species indirectly through the flexor plate), while those of the foot are the tendons of insertion of one or more of the following muscles: $\mathrm{m}$. flexor digitorum communis (which tendon of insertion forms the aponeurosis plantaris), $m$. flexor accessorius, $m$. tibialis posterior, and $\mathrm{mm}$. flexores breves superficiales (e.g., Dunlap, 1960; Burton, 2004; this study). Some muscles (usually the $\mathrm{mm}$. lumbricales) arise via tendons from some of these grayshaded elements, including the $\mathrm{m}$. flexor digitorum communis, $m$. flexor accessorius, flexor plate, aponeurosis plantaris, $\mathrm{mm}$. flexores breves superficiales, and tendines superficiales; these tendons are also gray shaded and justified on the same grounds.

When the muscles of the same group are adjacent or partially overlapping, their adjacent edges might not be clearly delimited in the edited image. To solve this problem, we used a thin line to delimit the shading of each individual element (fleshy portion of muscles, tendons, and ligaments). The fleshy portion of unidentified muscles are shaded black with low opacity, and addressed in the corresponding figure legend.

Finally, the model figures of Triprion petasatus are edited to show all muscle layers simultane- ously (i.e., in the figure of the first layers, the muscles that are partially visible from the successive deeper layers are also shaded with their respective predefined color). For the remaining figures of the study, only the exposed layer is edited (unless deeper layers are required for specific discussions), and the colors employed for each muscle group are the same as in T. petasatus.

\section{Character Evolution}

On the basis of our morphological analyses, we delimited transformation series following the character concept of Grant and Kluge (2004). All but one of our characters are binary, presence/ absence characters. The sole multistate character (char. 9) is treated as nonadditive (unordered) due to the absence of developmental or other evidence to establish nested homology. We then performed Fitch (1971) optimization of the characters on the phylogenetic hypothesis of Jetz and Pyron (2018) using the software TNT version 1.5 (Goloboff and Catalano, 2016). When relevant, we also discuss the implications of alternative character optimizations derived from competing phylogenetic hypotheses.

\section{RESULTS}

\section{DESCRIPTION OF THE HAND AND Foot Musculature of Triprion petasatus}

Palmar Surface of the Hand: First Layer (pl. 2A)

TENDINES SUPERFICIALES: The tendo superficialis digiti III originates from the reduced flexor plate. The tendo superficialis digiti IV originates from the medial slip of the $\mathrm{m}$. flexor digitorum communis and from a portion of the $\mathrm{m}$. flexor accessorius. The tendo superficialis digiti $V$ has three origins: from the flexor plate, and from the medial and lateral slips of the $\mathrm{m}$. flexor digitorum communis. The tendines superficiales digitorum III-V are distally bifid (not shown, hidden by integument) as they reach the insertion on the palmar surface of the distal phalanx of their respective digit. 
Presumed function: To flex digits III-V through the action of the $\mathrm{m}$. flexor digitorum communis and $\mathrm{m}$. flexor accessorius.

Comments: A tendo superficialis of digit II (tendo superficialis indicis) also occurs in some species (see char. 1).

M. FLEXOR INDICIS SUPERFICIALIS PROPRIUS: This muscle originates medially from distal carpal 3-4-5 and inserts via a long tendon. This tendon attaches to the palmar surface of the distal phalanx of digit II and is distally bifid (not shown, hidden by integument).

Presumed function: Flexes the distal phalanx of digit II; a component of adduction might occur in some species (Manzano et al., 2008: 302, 304).

M. CAPUT PROFUNDUM DIGITI III: This muscle is lateral to the tendo superficialis digiti III and originates via a short tendon from distal carpal 3-4-5; it inserts broadly along the dorsal surface of the tendo superficialis digiti III. Distally to this broad insertion, the $\mathrm{m}$. caput profundum digiti III and the tendo superficialis digiti III fuse into a single tendon that attaches to the palmar surface of the distal phalanx of digit III.

Presumed function: To flex the distal phalanx of digit III.

MM. LUMBRICALES LONGI: The mm. lumbricales longi digitorum IV and $\mathrm{V}$ are composed of a medial and lateral slip with origins from the tendines superficiales digitorum IV and V, respectively. The fleshy portion of the medial slip of the $\mathrm{m}$. lumbricalis longus digiti IV is approximately $1 / 4$ shorter than the lateral slip (in plate $2 \mathrm{~A}$ it appears to be more than $1 / 4$ shorter because the tendo superficialis hides a portion of it). The lengths of the fleshy portion of the medial and lateral slips of the m. lumbricalis longus digiti $\mathrm{V}$ are approximately equal, resulting in almost symmetric slips. Each of them inserts via a long, thin tendon on the proximal interphalangeal joint of digits IV and $\mathrm{V}$, respectively (only the proximal portion of the tendon of insertion of the lateral slip of the $\mathrm{mm}$. lumbricales longi digitorum IV and V are visible in the plate).

Presumed function: To flex the basal and second phalanges of digits IV and V.
Comments: Some species also possess an $\mathrm{m}$. lumbricalis longus in digit III (see char. 2).

Palmar Surface of the Hand: Second Layer (pl. 2B)

MM. LUMBRICALES BREVES: The m. lumbricalis brevis indicis and $\mathrm{m}$. lumbricalis brevis digiti III each originate via a tendon from the medial side of distal carpal 3-4-5 and insert via a short tendon on the medial and palmar surface of the metacarpophalangeal joint of their respective digits. The medial $\mathrm{m}$. lumbricalis brevis digiti IV originates from the tendo superficialis digiti IV proximal to the medial slip of the $\mathrm{m}$. lumbricalis longus digiti IV; it has a fleshy insertion on the mediodistal end of metacarpal IV (not shown) and tendinous insertion on the medial and palmar surface of the metacarpophalangeal joint of digit IV. The medial $\mathrm{m}$. lumbricalis brevis digiti $\mathrm{V}$ originates via a flat, wide, and discontinuous tendon from three points on distal carpal 3-4-5: medially at the point of origin of the m. contrahentis digiti $\mathrm{V}$, intermediary at the level of the lateral margin of the tendo superficialis digiti IV, and laterally at the level of the lateral margin of the tendo superficialis digiti $\mathrm{V}$; it inserts distally on the medial and palmar surface of metacarpal $\mathrm{V}$ via a short tendon. The lateral $\mathrm{m}$. lumbricalis brevis digiti $\mathrm{V}$ is composed of one slip that originates from distal carpal 3-4-5 and a second slip from the integument; they converge proximally into a single body that inserts on the lateropalmar surface of the metacarpophalangeal joint.

Presumed function: To flex the digits through the flexion of the basal phalanges and associated metacarpals.

Comments: The lateral $\mathrm{m}$. lumbricalis brevis digiti IV is absent in this species.

Palmar Surface of the Hand: Third Layer (pl. 2C)

Mm. contrahentes digitorum: The $\mathrm{m}$. contrahentis indicis has a tendinous origin from the mediodistal portion of the distal carpal 3-4-5 and inserts on the lateropalmar surface of the basal phalanx of digit II. The m. contrahentis digiti $\mathrm{V}$ originates via tendon, laterally to the $\mathrm{m}$. contrahentis indicis on distal carpal 3-4-5, and 
has a fleshy insertion on the lateropalmar surface of the distal half of metacarpal V.

Presumed function: The muscle of digit II flexes the basal phalanx, and the muscle of digit $\mathrm{V}$ adducts metacarpal V; a component of opposition of digit $\mathrm{V}$ cannot be discarded.

Comments: The mm. contrahentes of digits III and IV are present in some species (see Discussion).

Palmar Surface of the Hand: Fourth Layer (pl. 2D)

M. PRONATOR QUADRATUS: This muscle originates from the ulnare and radioulna via a very short tendon and inserts on the prepollex, proximally and medially to the insertion of the $\mathrm{m}$. adductor pollicis.

Presumed function: To abduct the prepollex (but see Discussion).

M. CONTRAHENTIS CAPUT LONGUS DISTALIS: This is a short muscle that originates from the ulnare and inserts on distal carpal 3-4-5.

Presumed function: This muscle is better developed, more superficial, and has a different fiber orientation in the early diverging anuran clades; its function is unclear and possibly changes over the evolutionary history of the group. Gaupp (1896: 168) suggested, on the basis of Pelophylax, rotation (in the pronation sense) and adduction of the radial side of the hand. The muscle of Triprion petasatus (as in most neobatrachians) is similar to that of Pelophylax, and Gaupp's comments are applicable to this species as well.

M. ADDUCTOR POLLICIS: The origin is tendinous from distal carpal 3-4-5, just medial to the origin of the medial $\mathrm{m}$. flexor indicis brevis profundus, and the insertion is on the palmar and distal surface of the prepollex.

Presumed function: Adducts the prepollex (but see Discussion).

MM. FLEXORES BREVES PROFUNDI: The medial $m$. flexor indicis brevis profundus has a tendinous origin from the medial region of distal carpal 3-4-5 and a fleshy insertion on the mediopalmar surface of metacarpal II and the connective tissue sheath or ligament that connects the distal portion of the prepollex to the mediodistal end of metacarpal II. The mm. flexores breves profundi digitorum III and IV each originate via a single tendon from the distal end of distal carpal 3-4-5. The former has a fleshy insertion on the lateropalmar surface of metacarpal III, and laterally a tendinous insertion on the metacarpophalangeal joint and proximally on the basal phalanx. The $\mathrm{m}$. flexor brevis profundus digiti IV has a fleshy insertion on the lateropalmar surface of metacarpal IV and a tendinous insertion on the lateropalmar surface of the metacarpophalangeal joint. The insertion of the mm. flexores breves profundi digitorum III and IV are ventral to the $\mathrm{mm}$. intermetacarpales II and III, respectively.

Presumed function: Digit II: to flex the digit through the metacarpal, also generating opposition; digits III-IV: to flex the digit through the flexion of the metacarpal and basal phalanx.

Comments: The lateral muscle of the $\mathrm{m}$. flexor indicis brevis profundus and the $\mathrm{m}$. flexor brevis profundus digiti $\mathrm{V}$ are absent in this species.

M. AbDuctor Digiti Minimi AND M. ABdUCTOR SECUNDUS DIGITI V: The $\mathrm{m}$. abductor digiti minimi is fused with the $\mathrm{m}$. abductor secundus digiti $V$. This fused muscle originates from the distal end of the radioulna and ulnare (topologically corresponding to the $\mathrm{m}$. abductor secundus digiti $\mathrm{V}$ of other species in which these muscles are distinct) and lateral surface of distal carpal 3-4-5 (topologically corresponding to the origin of the $\mathrm{m}$. abductor digiti minimi). The insertion is fleshy and covers the complete lateropalmar surface of metacarpal $\mathrm{V}$ (the portion of the insertion on the proximal end of metacarpal $\mathrm{V}$ corresponds topologically to the m. abductor secundus digiti V).

Presumed function: To abduct digit V through its metacarpal.

Comments: When distinct, the $\mathrm{m}$. abductor digiti minimi and $\mathrm{m}$. abductor secundus digiti $\mathrm{V}$ usually originate on the flexor plate and/or distal carpals and the ulnare and radioulna, respectively, and the insertion of the m. abductor secundus digiti $\mathrm{V}$ is usually restricted to the 
proximal-most portion of metacarpal V (e.g., Burton, 1998a: fig. 1C, 1D).

MM. FLEXORES DIGITORUM MINIMI: The mm. flexores minimi digitorum III and IV have a fleshy origin on the mediopalmar surface of metacarpals III and IV, dorsal to the origins of the $\mathrm{mm}$. intermetacarpales I and II, respectively. The $\mathrm{m}$. flexor minimus digiti $\mathrm{V}$ has two points of origin (both fleshy and ventral to the m. intermetacarpalis III), medially from the proximal and lateropalmar portion of metacarpal IV, and laterally from the proximal and mediopalmar portion of metacarpal V. Each of the $\mathrm{mm}$. flexores minimi digitorum III-V inserts via a tendon on the palmar surface of the basal phalanx of its respective digit. The fibers of the $\mathrm{mm}$. flexores minimi digitorum IV and $V$ reach distally the proximal end of the basal phalanx of digits IV and $\mathrm{V}$, respectively.

Presumed function: To flex the basal phalanx.

Comments: An m. flexor minimus is present in digit II of some species (see char. 8).

MM. InTERMETACARPALES: These are transversal muscles that connect the metacarpals. The $\mathrm{mm}$. intermetacarpales I, II, and III originate from metacarpals III, IV, and V and insert on metacarpals II, III, and IV, respectively. The $\mathrm{m}$. intermetacarpalis I has an additional insertion on the lateropalmar surface of the metacarpophalangeal joint of digit II.

Presumed function: To adduct the fingers; depending on their specific morphology and topology in each taxon, some component of abduction/ adduction might occur. In the case of Triprion petasatus, the m. intermetacarpalis I probably adducts and rotates metacarpal II. See also Burton (1998b: 2) for different functions of these muscles (closing, opening, and rotating the palm) in relation to their changing insertion points.

MM. INTERPHALANGEI: The mm. interphalangei digitorum IV and V consist of medial and lateral slips, equally developed, with fleshy origins on the medial and lateral palmar surface of the basal phalanx of digits IV and V, respectively. Each slip of the mm. interphalangei digitorum
IV and $\mathrm{V}$ inserts via an independent tendon on the second phalanx of digits IV and V, respectively.

Presumed function: To flex the second phalanx.

Comments: The $\mathrm{m}$. interphalangeus indicis and $\mathrm{m}$. interphalangeus digiti III are absent in this species.

Palmar Surface of the Hand: Muscles Unassigned to Layer (pl. 2B)

M. FLEXOR DIGITORUM COMMUNIS: This muscle originates from the humerus and is divided into medial and lateral slips. The medial slip has three distinct portions distally, one that inserts on the flexor plate and two others that connect to the tendines superficiales digitorum IV-V. The lateral slip connects to the tendo superficialis digiti $\mathrm{V}$.

Presumed function: To flex the digits III-V through the flexor plate and tendines superficiales; and to flex the wrist (at least in arboreal frogs with a similar morphological configuration to Triprion petasatus), as reported by Manzano et al. (2008: 303).

M. FLEXOR ACCESSORIUS: This muscle originates from the laterodistal end of the radioulna via a short tendon; it inserts on the flexor plate and also connects to the tendo superficialis digiti IV via a discrete tendon.

Presumed function: Possibly to flex digits III-IV through the flexor plate and tendines superficiales. However, as reported by Manzano et al. (2008: 304) for arboreal hylid species, adduction of digit $\mathrm{V}$ and rotation of the hand might be the main function in species with similar morphological configuration, as occurs in Triprion petasatus. See also Gaupp (1896: 159), who studied a nonarboreal species.

FleXOR Plate: This is a thin, tendinous sheet formed by the insertion of portions of the medial slip of the $\mathrm{m}$. flexor digitorum communis and the $\mathrm{m}$. flexor accessorius. The flexor plate warps the tendons of insertion of the $\mathrm{m}$. flexor digitorum communis and the $\mathrm{m}$. flexor accessorius that connect to the tendines superficiales of dig- 
its IV and V, leaving them free to slide within it. The flexor plate connects to the tendo superficialis digiti $\mathrm{V}$ and is the sole point of origin of the tendo superficialis digiti III.

Presumed function: See m. flexor digitorum communis and $\mathrm{m}$. flexor accessorius. It serves as a fixed point of origin of the superficial flexor muscles (mm. lumbricales breves and mm. lumbricales longi), which mostly occur in nonarboreal species and especially in early diverging anuran clades (more flexor muscles originating on the flexor plate occur in these clades). This fixed point of origin does not occur in Triprion petasatus and most arboreal clades due to their reduced flexor plate.

Comments: The flexor plate is usually absent or reduced (as in T. petasatus) in arboreal clades and well developed-sometimes with an embedded sesamoid-in other clades. Burton's ligament (which joins the distal carpals with the flexor plate or tendo superficialis digiti V) is absent in this species.

Dorsal Surface of the Hand: First Layer (pl. 3A)

M. EXTENSOR DIGITORUM: The $\mathrm{m}$. extensor digitorum originates from the humerus, extends dorsad along the radioulna, and inserts via three slips on metacarpals III, IV, and V. The insertion on metacarpal III is tendinous and also receives some medial fibers of the slip from the radioulna of the $\mathrm{m}$. extensor brevis superficialis digiti III. The insertion on metacarpal IV is almost fleshy, via a wide, very short tendon, and it also receives some fibers of the slip from the radioulna of the $m$. extensor brevis superficialis digiti IV. The insertion on metacarpal $\mathrm{V}$ is tendinous, just distal to the $\mathrm{m}$. abductor brevis digiti $\mathrm{V}$.

Presumed function: To extend digits III-V through the metacarpals and possibly to extend the wrist (at least in arboreal frogs with a similar morphological configuration to Triprion petasatus).

Dorsal Surface of the Hand: Second Layer (pl. 3B)

M. ABDuctor pollicis longus: This is a well-developed muscle with a broad fleshy origin on the dorsal surface of the radioulna; it inserts via a flat tendon (only the distal portion is visible in plate $3 \mathrm{~B}$; the tendon of insertion arises more proximally and is hidden dorsally by the fleshy portion). The tendon inserts on the fascia of the $\mathrm{m}$. extensor indicis brevis medius and an "extensor brevis-like" muscle (see below) and continues on to attach to metacarpal II, just distal to the insertion of these extensor muscles.

Presumed function: To abducts digit II through its metacarpal.

MM. EXTENSORES DIGITORUM BREVES SUPERFICIALES: The $\mathrm{m}$. extensor indicis brevis superficialis has a continuous origin covering the radiale and a short ligament that joins the radiale and ulnare dorsally; the origin on the radiale is lateral to the tendon of insertion of the $\mathrm{m}$. extensor carpi radialis that attaches to element $\mathrm{Y}$. The medial fibers insert via a common tendon with an "extensor brevis-like" muscle (see below) on the dorsal surface of metacarpal II, and the lateral fibers insert via tendon on the metacarpophalangeal joint of digit II.

The $\mathrm{m}$. extensor brevis superficialis digiti III is composed of two slips, one that originates on the radioulna via a common tendon with the slip from radioulna of the $\mathrm{m}$. extensor brevis superficialis digiti IV, and a second one that originates on the ulnare. Both slips have fleshy insertions on the dorsal surface of metacarpal III, while some medial fibers of the slip from the radioulna attach along the tendon of insertion of the $\mathrm{m}$. extensor digitorum (which also attaches to metacarpal III).

The $\mathrm{m}$. extensor brevis superficialis digiti IV is composed of three slips defined by their points of origin: radioulna, ulnare, and distal carpal 3-4-5. The radioulna slip has a tendinous origin and fleshy insertion on the dorsal surface of metacarpal IV (some fibers also attach to the short tendon of insertion of the $\mathrm{m}$. extensor digitorum) and the metacarpophalangeal joint. The slip from the ulnare has a fleshy insertion on the dorsal surface of metacarpal IV, contiguous with the slip from the radioulna. The slip from the distal carpal 3-4-5 has its main origin on distal 
carpal 3-4-5, while a few fibers also arise from the fascia of the distal end of the $\mathrm{m}$. extensor carpi ulnaris at its insertion point on distal carpal 3-4-5; it inserts via the tendon of insertion of the lateral $\mathrm{m}$. dorsometacarpalis proximalis digiti IV, which attaches to the proximal interphalangeal joint.

The $\mathrm{m}$. extensor brevis superficialis digiti $\mathrm{V}$ is reduced in comparison to that of the other digits. It originates from the distal carpal 3-4-5 and inserts via a long tendon on the metacarpophalangeal joint of digit $\mathrm{V}$.

An "extensor brevis-like" muscle occurs on digit II (hidden by the m. abductor pollicis longus and not visible in the plate). Although it is unclear if it belongs to the superficiales or medii muscle groups, we arbitrarily assign it to the former group for expediency. It has a fleshy origin on the tendon of insertion of the $\mathrm{m}$. extensor carpi radialis at the level of the distal end of the radioulna. It inserts on metacarpal II via a common tendon with a portion of the $\mathrm{m}$. extensor indicis brevis superficialis.

Presumed function: To extend the digits through their respective metacarpal and basal phalanx (digits II and V), metacarpal (digit III), metacarpal, and basal and second phalanx (digit IV).

Dorsal Surface of the Hand: Third Layer (pl. 3C)

MM. EXTENSORES DIGITORUM BREVES MEDII: The $\mathrm{m}$. extensor indicis brevis medius originates from the radiale, medial to the tendon of insertion of the $\mathrm{m}$. extensor carpi radialis that attaches to element $\mathrm{Y}$. It has a fleshy insertion on the dorsal surface of metacarpal II.

The $\mathrm{m}$. extensor brevis medius digiti III is composed of two slips, one originating on element $\mathrm{Y}$ and the other on the radiale. The former inserts via a long tendon on the metacarpophalangeal joint that is shared with the medial portion of the slip from the radiale; the slip from the radiale also has a fleshy insertion on the dorsal surface of metacarpal III.

The $\mathrm{m}$. extensor brevis medius digiti IV originates from element $\mathrm{Y}$ and the radiale without forming distinct heads or slips; only a few lateral fibers arise from the radiale. It inserts via a long tendon attached to the dorsomedial surface of the metacarpophalangeal joint of digit IV.

Presumed function: To extend the digits through the metacarpal (digit II) and the metacarpal and basal phalanx (digits III and IV).

Dorsal Surface of the Hand: Fourth Layer (pl. 3D)

M. ABDUCTOR INDICIS BREVIS DORSALIS: The $\mathrm{m}$. abductor indicis brevis dorsalis has a fleshy origin on element $\mathrm{Y}$ and a separate fleshy insertion for each of its two slips. The main slip inserts on the dorsal surface of the prepollex, while a very tiny slip inserts on the proximal and medial surface of metacarpal II.

Presumed function: To abduct metacarpal II by its direct insertion (extremely reduced in Triprion petasatus) and possibly an indirect action through the well-developed insertion on the dorsal surface of the prepollex, which is joined by connective tissue to metacarpal II and might act as a single structure in this species.

M. ABDUCtor BREVIS Digiti V: The $\mathrm{m}$. abductor brevis digiti $\mathrm{V}$ has a fleshy origin from the dorsolateral region of distal carpal 3-4-5 and a fleshy insertion on the lateral surface of metacarpal V.

Presumed function: To abduct metacarpal V.

MM. DORSOMETACARPALES PROXIMALES: There are at least eight muscles in this group, four on each side of each digit, some of which are subdivided into discrete slips. In addition to these eight muscles, there are intermediate portions on digits III, IV, and V, for which it is unclear if they are distinct muscles or slips of the lateral or medial muscles; for expediency we treat them as distinct muscles.

The medial $\mathrm{m}$. dorsometacarpalis indicis proximalis is composed of a medial and a lateral slip, both with fleshy origins from the medial and dorsal surface of metacarpal II, respectively. The slips converge to form a common tendon of insertion that attaches to the interphalangeal joint/distal phalanx. The lateral $\mathrm{m}$. dorsometacarpalis indicis proximalis has a fleshy origin from distal carpal 2 and the dorsal surface of 
metacarpal 1I; it inserts on the interphalangeal joint/distal phalanx of digit II.

The medial $\mathrm{m}$. dorsometacarpalis proximalis digiti III is composed of two slips, one from distal carpal 2 and the other from the dorsomedial surface of metacarpal III (these slips are not differentiable in the plate); they converge to form a common tendon of insertion that attaches broadly to the dorsal and medial surfaces of the interphalangeal joint/distal phalanx of digit III. The intermediate $\mathrm{m}$. dorsometacarpalis proximalis digiti III has a wide fleshy origin on the dorsal surface of metacarpal III and inserts via a short, wide tendon on the metacarpophalangeal joint of digit III. The lateral $\mathrm{m}$. dorsometacarpalis proximalis digiti III has a fleshy origin on the lateral surface of metacarpal III and inserts on the dorsolateral surface of the interphalangeal joint/distal phalanx of digit III.

The medial $\mathrm{m}$. dorsometacarpalis proximalis digiti IV is composed of a single slip with a fleshy origin from the proximal and lateral surface of metacarpal III; it inserts medially on the distal interphalangeal joint/distal phalanx of digit IV. The intermediate $\mathrm{m}$. dorsometacarpalis proximalis digiti IV has a fleshy origin on the dorsal surface of metacarpal IV and inserts on the metacarpophalangeal joint of digit IV via a short, wide tendon. The lateral $\mathrm{m}$. dorsometacarpalis proximalis digiti IV is composed of two slips, one with a fleshy origin from metacarpal IV and the other with a tendinous origin from the dorsomedial and proximal surface of metacarpal $\mathrm{V}$. The slip from metacarpal IV inserts via a common tendon with the medial portion of the slip from metacarpal $\mathrm{V}$; this tendon of insertion splits into medial and lateral tendons and attaches to the proximal interphalangeal joint of digit IV. The slip from metacarpal $\mathrm{V}$ also inserts on the distal interphalangeal joint/distal phalanx of digit IV via an independent tendon.

The medial $\mathrm{m}$. dorsometacarpalis proximalis digiti $\mathrm{V}$ is composed of two slips, one with a fleshy origin from the proximal and lateral surface of metacarpal IV and a second one with a fleshy and tendinous origin from the dorsome- dial surface of metacarpal $V$. The fibers of the slip from metacarpal IV extend along more than half of the basal phalanx of digit $\mathrm{V}$, and inserts with the medial $\mathrm{m}$. dorsometacarpalis distalis digiti $\mathrm{V}$ on the distal interphalangeal joint/distal phalanx of digit $\mathrm{V}$. The slip of the medial $\mathrm{m}$. dorsometacarpalis proximalis digiti $\mathrm{V}$ with origin from metacarpal $\mathrm{V}$ inserts on the medial margin of the proximal interphalangeal joint of digit $\mathrm{V}$. The intermediate $\mathrm{m}$. dorsometacarpalis proximalis digiti V has a wide origin, mostly fleshy, on the dorsal surface of metacarpal V, with some proximal fibers also arising via a tendon; it inserts on the metacarpophalangeal joint of digit $\mathrm{V}$ via a short and wide tendon. The lateral $\mathrm{m}$. dorsometacarpalis proximalis digiti $\mathrm{V}$ has a fleshy origin from the dorsolateral surface of metacarpal $\mathrm{V}$ and inserts on the lateral margin of the proximal interphalangeal joint of digit $\mathrm{V}$.

Presumed functions: To extend the phalanges and assist in the detachment of the adhesive discs; the presence of intermediate muscles in digits III-V might result in the extension of the digits through the metacarpals and basal phalanges due to their insertion on the metacarpophalangeal joints:

MM. DORSOMETACARPALES DISTALES: Four muscles of this group are present: lateral muscles on digits III-V and a medial muscle on digit V. The lateral $\mathrm{m}$. dorsometacarpalis distalis digiti III originates from the laterodistal end of metacarpal III; its fibers extend distad approximately $3 / 4$ of the length of the basal phalanx and insert on the interphalangeal joint/distal phalanx of digit III. The lateral $\mathrm{m}$. dorsometacarpalis distalis digiti IV originates from the laterodistal end of metacarpal IV and the proximal and lateral surface of the basal phalanx; its fibers reach the proximal interphalangeal joint of digit IV. The medial $\mathrm{m}$. dorsometacarpalis distalis digiti $\mathrm{V}$ originates from the mediodistal end of metacarpal $\mathrm{V}$ and the proximal and medial surface of the basal phalanx; its fibers extend distad between $3 / 4$ and $4 / 5$ of the second phalanx of digit $V$. The lateral $\mathrm{m}$. dorsometacarpalis distalis digiti $\mathrm{V}$ originates from the laterodistal end of metacar- 
pal $\mathrm{V}$ and the proximal and lateral surface of the basal phalanx; its fibers extend distad along approximately $3 / 4$ of the second phalanx of digit $\mathrm{V}$. The $\mathrm{mm}$. dorsometacarpales distales digitorum IV and V insert on the distal interphalangeal joint/distal phalanx of digits IV and V, respectively.

Presumed function: To extend the distal phalanges and detach the adhesive discs.

Comments: There are at most seven muscles of this group in some species: medial and lateral (on digits II, IV, and V), and lateral (digit III). Burton (1998c) reported the presence of a medial muscle on digit III in a few species, but we did not observe it in any of those species we also studied.

Dorsal Surface of the Hand: Muscles Unassigned to Layer (pl. 3A)

M. EXTENSOR CARPI ULNARIS: This muscle originates from the humerus, runs laterad to the $\mathrm{m}$. extensor digitorum along the dorsal surface of the radioulna, and inserts via a very short tendon on the ulnare and distal carpal 3-4-5.

Presumed function: To abduct and extends the hand (see Gaupp, 1896: 154).

Comments: This muscle is not studied here (see Materials and Methods for the targeted muscles), but it is briefly described because its removal is required to expose the origin of the $\mathrm{mm}$. extensores breves superficiales on the laterodistal end of radioulna, ulnare, and most postaxial distal carpals.

Plantar Surface of the Foot: First Layer (pl. 4A)

Tendines superficiales: The tendo superficialis praehallucis arise as a broad, flat tendon that attaches to the prehallux; its morphology more closely resembles an extension of the aponeurosis plantaris that attaches to the prehallux than the tendines superficiales of the digits. The tendo superficialis hallucis and tendo superficialis digiti II originate from the distal end of the aponeurosis plantaris; they attach to the plantar surface of the distal phalanx of digits I and II, respectively. The tendines superficiales digitorum
III-V originate from the single tendon of insertion of the $\mathrm{mm}$. flexores breves superficiales and insert on the plantar surface of the distal phalanx of digits III-V, respectively.

Presumed function: The tendines superficiales are distal portions of the tendons of insertion of other muscles, and there are a multiplicity of flexion forces produced by the series of muscles that are related to them: (1) $\mathrm{m}$. flexor digitorum communis: tendon of insertion forms aponeurosis plantaris, from which tendo praehallucis and tendines superficiales of digits I and II arise; also wraps partially and adheres to tendon of insertion of mm. flexores breves superficiales; (2) $\mathrm{mm}$. flexores breves superficiales: tendon of insertion splits into tendines superficiales digitorum III-V; (3) m. flexor accessorius: inserts distally and medially on aponeurosis plantaris; and (4) m. tibialis posterior: inserts on aponeurosis plantaris. Their differential contractions may act through the aponeurosis plantaris in different ways and intensities. Its concerted action through their integration by the aponeurosis plantaris generates a simultaneous flexion of the whole plantar surface (e.g., during swimming and jumping).

Comments: Although the tendo superficialis praehallucis belongs to this group, it is usually removed and studied together with the removal of the aponeurosis plantaris on the third layer.

MM. LUMBRICALES LONGI: The $\mathrm{m}$. lumbricalis longus digiti III originates from the aponeurosis plantaris and a thin tendon arising on the proximal end of the tendo superficialis digiti III; it inserts via a medial tendon (with respect to the tendo superficialis digiti III) on the medioplantar region of the proximal interphalangeal joint of digit III. The m. lumbricalis longus digiti IV has a very short tendon of origin (almost fleshy) from the base of the tendo superficialis digiti IV, adjacent (distal) to the splitting point of the single tendon of insertion of the $\mathrm{mm}$. flexores breves superficiales into the tendines superficiales digitorum III-V. It inserts via a medial tendon (relative to the tendo superficialis digiti IV) on the medioplantar region of the proximal 
interphalangeal joint of digit IV. The m. lumbricalis longus digiti $V$ originates from the proximal end of the tendo superficialis digiti $\mathrm{V}$ and inserts via a lateral tendon (relative to the tendo superficialis digiti V) on the lateroplantar region of the proximal interphalangeal joint of digit $\mathrm{V}$.

Presumed function: To flex the basal and second phalanges of digits III-V.

Comments: Some species also possess an $\mathrm{m}$. lumbricalis longus in digit II.

M. LUMBRICALIS LONGISSIMUS DIGITI IV: The $\mathrm{m}$. lumbricalis longissimus digiti IV originates via a long tendon from the same point as the $\mathrm{m}$. lumbricalis longus digiti IV. It inserts via a medial tendon (relative to the tendo superficialis digiti IV) on the medioplantar region of the second interphalangeal joint of digit IV (the tendon of insertion is partially fused to the connective tissue at the level of the metatarsophalangeal joint and the proximal interphalangeal joint; it does not slide freely through these joints).

Presumed function: To flex the second and third phalanx of digit IV; in this species it also flexes the metatarsal and basal phalanx since its tendon of insertion is partially fused to the connective tissue of the metatarsophalangeal and proximal interphalangeal joints (from which it is free in most species).

Plantar Surface of the Foot: Second Layer (pl. 4B)

Mm. Lumbricales Breves: This muscle group is represented by a single medial muscle in digits I-III and a medial and lateral muscle in digits IV-V. The $\mathrm{m}$. lumbricalis brevis hallucis and $\mathrm{mm}$. lumbricales breves digitorum II-III originate from the distal end of the aponeurosis plantaris; the origin of the former is fleshy, whereas those of digits II and III are tendinous. They insert via a short tendon on the medioplantar surface of the metatarsophalangeal joint of their respective digits.

The medial $\mathrm{m}$. lumbricalis brevis digiti IV has a tendinous origin from the distal end of the aponeurosis plantaris and inserts on the medioplantar surface of the metatarsophalangeal joint of digit IV. The lateral m. lumbricalis brevis digiti IV originates from the plantar cartilage (sensu Dunlap, 1960) via a common tendon with the medial slip of the medial $\mathrm{m}$. lumbricalis brevis digiti V (additionally, some distal and superficial fibers originates from the integument); it inserts via tendon widely on the central and lateral plantar surface of the metatarsophalangeal joint of digit IV.

The medial $\mathrm{m}$. lumbricalis brevis digiti $\mathrm{V}$ is composed of a medial and a lateral slip. The medial slip originates from the plantar cartilage via a long common tendon with the lateral $\mathrm{m}$. lumbricalis brevis digiti IV and a thin tendon from the tendon of insertion of $\mathrm{mm}$. flexores breves superficiales (at level of its splitting point into the tendines superficiales digitorum III-V); it has a fleshy insertion on metatarsal V (laterally to the $\mathrm{m}$. flexor minimus digiti V; not shown in plate) and a short tendinous insertion on the medioplantar region of the metatarsophalangeal joint of digit $\mathrm{V}$. The lateral slip of the medial $\mathrm{m}$. lumbricalis brevis digiti $\mathrm{V}$ has a short tendon of origin from the plantar cartilage (partially shared with the medial slip) and inserts via a long, flat tendon on metatarsal V (laterally to the $\mathrm{m}$. flexor minimus digiti V; not shown in plate) and widely on the plantar surface of the metatarsophalangeal joint of digit V. The lateral $\mathrm{m}$. lumbricalis brevis digiti $\mathrm{V}$ originates via a tendon from the plantar cartilage and inserts via a short tendon on the lateroplantar surface of the metatarsophalangeal joint of digit V.

Presumed function: To flex the digits through the metatarsals and basal phalanges; the lateral muscle of digit $\mathrm{V}$ probably produces some component of abduction as well.

Comments: A lateral muscle in digit III occurs in some species of early diverging clades.

Plantar Surface of the Foot: Third Layer (pl. 4C)

MM. CONTRAHENTES Digitorum PEDIS: Only the $\mathrm{m}$. contrahentis pedis hallucis is present. It originates via a tendon from distal tarsal 2-3, and inserts via a tendon on the lateroplantar surface of the basal phalanx of digit I. 
Presumed function: To flex the basal phalanx.

Comments: Up to four (digits I-III, V) or five muscles (one per digit) are present in early diverging anuran clades.

Plantar Surface of the Foot: Fourth Layer (pl. 4D)

M. FLEXOR HALLUCIS ACCESSORIUS: The $m$. flexor hallucis accessorius has a tendinous origin from distal tarsal 2-3, medially to the m. contrahentis pedis hallucis, and has a fleshy insertion on the medioplantar surface of metatarsal I.

Presumed function: To flex digit I through the metatarsal.

MM. FLEXORES BREVES PROFUNDI: The $\mathrm{mm}$. flexores breves profundi digitorum II and III arise via a common tendon from the lateroplantar and distal end of the fibulare, as do the $\mathrm{m}$. flexor brevis profundus digiti IV via an independent tendon. The $\mathrm{mm}$. flexores breves profundi digitorum IIIV have fleshy insertions on the lateroplantar surface of metatarsals II, III, and IV, respectively.

Presumed function: To flex digits II-IV through their metatarsals; a component of abduction may also be present.

Comments: The $\mathrm{m}$. flexor hallucis brevis profundus is absent in this species.

M. ABDUCTOR BREVIS PLANTARIS DIGITI V: The $\mathrm{m}$. abductor brevis plantaris digiti $\mathrm{V}$ has a tendinous origin from the distal end of the fibulare, just laterally to the origin of the $\mathrm{mm}$. flexores breves profundi, and has a fleshy insertion on the entire lateroplantar surface of metatarsal V.

Presumed function: To abduct metatarsal V.

MM. FLEXORES DIGITORUM MINIMI: The mm. flexores minimi digitorum II-V have a fleshy origin on metatarsals II-V, respectively, and insert via tendon on the plantar surface of the basal phalanx of their respective digit. The $\mathrm{mm}$. flexores minimi digitorum II-IV have their origins dorsally to the mm. intermetatarsales I-III, respectively.

Presumed function: To flex the basal phalanx of digits II-V.

Comments: Some species also posses an $\mathrm{m}$. flexor minimus in digit I.
MM. intermetatarsales: The $\mathrm{mm}$. intermetatarsales I-III are transversal flat muscles, with fleshy origin from the medial margin of metatarsals II, III, and IV, and fleshy insertion on the lateral margin of metatarsals I, II, and III, respectively.

Presumed function: To join adjacent metatarsals; this function may be relevant during swimming (see Gaupp, 1896: 214).

Comments: The m. intermetatarsalis IV (that connects metatarsals IV and V) is absent in this species.

MM. INTERPHALANGEI: This group is represented by the $\mathrm{mm}$. interphalangei digitorum III, proximalis IV, distalis IV, and proximalis $\mathrm{V}$. All the $\mathrm{mm}$. interphalangei are composed of an equally developed medial and a lateral slip, except the $\mathrm{m}$. interphalangeus distalis digiti IV (which has the medial slip reduced in comparison with the lateral one). The medial and lateral slips of the $\mathrm{mm}$. interphalangei digitorum III, proximalis IV, and proximalis $\mathrm{V}$ have a fleshy origin on the medial and lateral plantar surface of the basal phalanx of digits III-V, respectively. They insert via tendon on the second phalanx of their respective digit. The medial and lateral slips of the m. interphalangeus distalis digiti IV have a fleshy origin on the medial and lateral plantar surface of the second phalanx, respectively, and insert via a tendon on the third phalanx of digit IV.

Presumed function: To flex the second (muscles of digits III, digit V, and proximal muscle of digit IV), and third phalanx (distal muscle of digit IV).

Comments: The intermediate slips of these four muscles, the $\mathrm{m}$. interphalangeus digiti II, and the $\mathrm{m}$. interphalangeus distalis digiti $\mathrm{V}$ are absent in this species.

Plantar Surface of the Foot: Muscles Unassigned to Layer (pl. 4C, D)

M. ABDUCtor praehallucis: This muscle has a similar position and morphology to the $\mathrm{m}$. abductor brevis plantaris hallucis, and most of its fibers are located medially to that muscle. The origin is fleshy from the medial border of the 
aponeurosis plantaris, and inserts on the prehallux.

Presumed function: To abduct the prehallux.

M. ABDUCTOR BREVIS PLANTARIS HALLUCIS: This muscle has a fleshy origin from the medial border of the aponeurosis plantaris, adjacent to the m. abductor praehallucis. It inserts via a long tendon on the medioplantar surface of metatarsal I.

Presumed function: To abduct metatarsal I.

APONEURosis PLANTARIS: The aponeurosis plantaris is a sheet of connective tissue covering most of the plantar surface. It is thicker centrally and originates as the distal expansion of the tendon of insertion of the $\mathrm{m}$. flexor digitorum communis.

Presumed function: See comments above for the tendines superficiales.

MM. FLEXORES BREVES SUPERFICIALES: Despite its plural designation, the $\mathrm{mm}$. flexores breves superficiales are composed of a single muscular body. It is located on the lateroplantar surface of the tarsus and is ventrally concealed by the aponeurosis plantaris. It originates from the ligamentum calcanei and inserts via a single tendon that splits into the tendines superficiales digitorum III-V.

Presumed function: To flex digits III-IV through the tendines superficiales; see also Comments for the tendines superficiales.

Comments: The fleshy portion is not visible in the plates; only its tendon of insertion is partially visible due to the transparency of the aponeurosis plantaris (depicted as a dashed line in pl. 4C). This muscle is divided into two or three slips, each with its own tendon of insertion, in some arboreal species.

M. FLEXOR ACCESSORIUS (PROXIMALIS AND DISTALIS): This muscle is composed of proximal (m. flexor accessorius proximalis) and distal ( $\mathrm{m}$. flexor accessorius distalis) portions. The $\mathrm{m}$. flexor accessorius proximalis arises via a fleshy origin from the distal end of the fibulare, passes dorsad to the distal end of the $\mathrm{mm}$. flexores breves superficiales, and inserts on the dorsal surface of the aponeurosis plantaris at the level of the prehallux.
The $\mathrm{m}$. flexor accessorius distalis has a fleshy origin from the plantar cartilage, passes dorsad to the tendon of insertion of the $\mathrm{mm}$. flexores breves superficiales, and inserts distally on the dorsal surface of the aponeurosis plantaris. Its insertion on the aponeurosis plantaris is at the level of the origin of the tendo superficialis digiti II, $\mathrm{m}$. lumbricalis brevis digiti III, $\mathrm{m}$. lumbricalis longus digiti III, and medial $\mathrm{m}$. lumbricalis brevis digiti IV (a distal portion inserting at the base of the tendo superficialis digiti II and $\mathrm{m}$. lumbricalis brevis digiti III is visible in pl. 4).

Presumed function: See the section on the tendines superficiales, above, and Gaupp (1896: 203).

M. Tibialis posterior: This single, large muscle originates from the ligamentum calcanei and has a fleshy insertion on the dorsal surface of the aponeurosis plantaris. It is located on the medioplantar surface of the tarsus, ventral to the $\mathrm{m}$. contrahentium caput longum. Its tendon of origin is independent of the tendon of origin of the $\mathrm{mm}$. flexores breves superficiales and the $\mathrm{m}$. contrahentium caput longum.

Presumed function: To flex the plantar surface and, possibly, digits I and II through its insertion on the dorsal and medial portion of the aponeurosis plantaris (which serves as origin for the tendines superficiales of digits I and II); see also the section on tendines superficiales above.

Comments: Not visible in plates.

M. CONTRAHENTIUM CAPUT LONGUM: This single muscle originates from the ligamentum calcanei on its medial side. It is located on the medioplantar surface of the tarsus and has a fleshy insertion along the distal $3 / 4$ of the ventral surface of the tibiale. Its origin is independent of the $\mathrm{m}$. tibialis posterior.

Presumed function: Plantar flexion and pronation (Gaupp, 1896: 200).

Comments: Not visible in plates.

M. INTEROSSEUS CRURIS: This is a bipinnate muscle with origins from the tibiale and the fibulare; the fibers converge into a tendon of insertion that attaches to element $\mathrm{Y}$.

Presumed function: Plantar adduction and pronation (Gaupp, 1896: 203). 
Comments: Only a distal portion of its tendon of insertion is visible in plate $4 \mathrm{D}$.

Dorsal Surface of the Foot: First Layer (pl. 5A)

M. EXTENSOR DIGITORUM LONGUS: This muscle arises from the distal end of the tibiofibula via a long tendon. Distally, it is differentiated into a medial and lateral slip, with a tendinous insertion on the dorsal surface of metatarsals II and III, respectively.

Presumed function: To extend digits II and III through its metatarsals.

Comments: Slips also attaching to digits I and/or IV-V occur in some species.

Dorsal Surface of the Foot: Second Layer (pl. 5B)

MM. EXTENSORES DIGITORUM BREVES SUPERFICIALES: There are five muscles of this group, one in digits I-III and two in digit IV. The $\mathrm{m}$. extensor brevis superficiales hallucis has a fleshy origin from the fibulare, which is proximally undifferentiated from the $\mathrm{m}$. extensor brevis superficialis digiti II. It has three insertions, a medial insertion via an aponeurosis on the prehallux, an intermediate insertion via a tendon on the medial and dorsal surface of metatarsal I, and a lateral insertion on the metatarsophalangeal joint of digit I, in common with the tendon of insertion of the m. extensor brevis medius hallucis. A few lateral and distal fibers of the $\mathrm{m}$. extensor brevis superficialis hallucis and $\mathrm{m}$. extensor brevis medius hallucis insert via a sheath of fascia on the medial surface of the medial $\mathrm{m}$. dorsometatarsalis proximalis digiti II (not shown). This sheath of fascia is proximally fused to the fascia of the medial $\mathrm{m}$. dorsometatarsalis proximalis digiti II.

The $\mathrm{m}$. extensor brevis superficialis digiti II has a fleshy origin from the fibulare. Medially it has a fleshy insertion on the medial and dorsal surface of metatarsal II, and laterally it inserts via two parallel tendons on the metatarsophalangeal joint of digit II, shared with the tendon of insertion of the $\mathrm{m}$. extensor brevis medius digiti II.

The $\mathrm{m}$. extensor brevis superficialis digiti III arises via a flat tendon from the distal end of the fibulare; the lateral portion of this tendon arises in common with the tendon of origin of the medial $\mathrm{m}$. extensor brevis superficialis digiti IV. It has three insertions: a fleshy insertion on metatarsal III, a wide medial tendon on the metatarsophalangeal joint, and a long lateral tendon on the proximal interphalangeal joint of digit III.

The $\mathrm{m}$. extensor brevis superficialis digiti IV is composed of equally developed medial and lateral muscles. Both have tendinous origins from the distal end of the fibulare. The tendon of the medial muscle is partially shared with the tendon of origin of the $\mathrm{m}$. extensor brevis superficialis digiti III, and the tendon of the lateral muscle is independent of other muscles. The medial and lateral muscles insert via a tendon on the metatarsophalangeal joint and proximal interphalangeal joint of digit IV, respectively.

Presumed function: To extend the digits through the metatarsals and basal phalanges of digits I-II and to extend the metatarsal, basal phalanx and second phalanx of digits III-IV.

Comments: Some species also posses an $\mathrm{m}$. extensor brevis superficialis in digiti V.

Dorsal Surface of the Foot: Third Layer (pl. 5C)

MM. EXTENSORES DIGITORUM BREVES MEDII: There are two muscles of this group, one on digit I and the other on digit II. The $\mathrm{mm}$. extensores breves medii hallucis and digiti II have a fleshy origin from the fused distal portions of the tibiale and fibulare. They insert via a common tendon with the $\mathrm{mm}$. extensores breves superficiales hallucis and digiti II, on the metatarsophalangeal joint of digits I and II, respectively. The m. extensor brevis medius digiti II has an additional medial fleshy insertion on the dorsal surface of metatarsal II.

Presumed function: To extend the metatarsal and basal phalanx of digits I and II.

Comments: The mm. extensores breves medii digitorum III and IV are absent in this species.

Dorsal Surface of the Foot: Fourth Layer (pl. 5D)

MM. DORSOMETATARSAles Proximales: There are 10 muscles of this group, with one 
medial and one lateral muscle for each digit, some of which are subdivided into discrete independent slips. In addition to these 10 muscles, there is an intermediate portion on digit V. As discussed for the analogous muscles of the hand, it is unclear whether it is a distinct muscle or a slip of the lateral muscle instead ${ }^{1}$; for the purpose of this account it will be treated as a distinct muscle of the $\mathrm{mm}$. dorsometatarsales proximales of digit $\mathrm{V}$.

The $\mathrm{mm}$. dorsometatarsales proximales of digit I comprise a medial and a lateral muscle. The medial $\mathrm{m}$. dorsometatarsalis hallucis proximalis has four slips with distinct origins (all fleshy) from: (1) the prehallux, (2) the distal half of the medial and dorsal surface of metatarsal I, (3) element Y, and (4) the dorsal and proximal surface of metatarsal I. These slips converge distally into a common tendon of insertion that attaches medially to the metatarsophalangeal joint of digit $\mathrm{I}$. The lateral $\mathrm{m}$. dorsometatarsalis hallucis proximalis is a single muscle that originates from the dorsolateral surface of metatarsal I; it inserts via a single tendon, which is distally bifurcated to attach to the dorsomedial and dorsolateral surfaces of the interphalangeal joint/ distal phalanx of digit I.

The medial $\mathrm{m}$. dorsometatarsalis proximalis digiti II has a tendinous origin from the proximolateral portion of metatarsal I and inserts medially on the metatarsophalangeal joint of digit II. The lateral $\mathrm{m}$. dorsometatarsalis proximalis digiti II has a tendinous origin from the tendon of origin of the medial $\mathrm{m}$. dorsometatarsalis proximalis digiti III proximally and a fleshy

${ }^{1}$ Burton (2004: 215), while describing the musculature of Hyla versicolor, reported that the lateral $\mathrm{m}$. dorsometatarsalis proximalis (as $\mathrm{m}$. extensor brevis profundus) "arises from the laterodorsal surface of the proximal end of Metatarsus V; some fibers insert on a short tendon to the base of Basal Phalanx V, whereas others insert by a long tendon that inserts on the dorsum of Penultimate Phalanx V." The insertion on the basal phalanx of digit $\mathrm{V}$ reported by Burton might represent a weakly developed and differentiated intermediate muscle or, alternatively, an additional insertion on the basal phalanx of the lateral muscle, as described and interpreted by Burton (2004). The muscle described here as an intermediate m. dorsometatarsalis proximalis is mostly distinct from the lateral muscle, and its origin is dorsally located on metatarsal $\mathrm{V}$. origin from the dorsolateral surface of metatarsal II distally; it inserts via a single tendon, which is distally bifurcated to attach to the dorsomedial and dorsolateral surfaces of the interphalangeal joint/distal phalanx of digit II.

The medial $\mathrm{m}$. dorsometatarsalis proximalis digiti III originates via a tendon from the proximolateral portion of metatarsal II. It inserts via a ventromedial tendon on the medial region of the metatarsophalangeal joint and a dorsolateral tendon on the medial region of the proximal interphalangeal joint of digit III. The lateral $\mathrm{m}$. dorsometatarsalis proximalis digiti III has a fleshy origin from the dorsolateral surface of metatarsal III; it inserts via a single tendon, distally bifid, on the dorsal surface of the distal interphalangeal joint/distal phalanx of digit III, in common with the insertion of the lateral $\mathrm{m}$. dorsometatarsalis distalis digiti III.

The medial $\mathrm{m}$. dorsometatarsalis proximalis digiti IV has a tendinous origin from the fused distal portions of the tibiale and fibulare. It is distally differentiated into a ventromedial portion that inserts via a short tendon on the medial region of the metatarsophalangeal joint and a dorsolateral portion that inserts via a long tendon on the medial surface of the distal interphalangeal joint/distal phalanx of digit IV. The lateral $\mathrm{m}$. dorsometatarsalis proximalis digiti IV originates proximally on the dorsal surface and distally on the dorsolateral surface of metatarsal IV. The fibers reach distally the proximal $1 / 4$ of the basal phalanx of digit IV, and inserts widely on the dorsal surface of the distal interphalangeal joint/distal phalanx, in common with the lateral $\mathrm{m}$. dorsometatarsalis distalis digiti IV.

The medial $\mathrm{m}$. dorsometatarsalis proximalis digiti $\mathrm{V}$ has a fleshy origin on the medial and dorsal surface of the distal half of metatarsal $\mathrm{V}$ and inserts medially on the distal interphalangeal joint/distal phalanx of digit $\mathrm{V}$, partially shared with the medial $\mathrm{m}$. dorsometatarsalis distalis digiti $\mathrm{V}$. The intermediate $\mathrm{m}$. dorsometatarsalis proximalis digiti $\mathrm{V}$ arises distally on the dorsal surface of metatarsal $\mathrm{V}$ between the medial and lateral muscles. It inserts via a short, 
wide tendon on the metatarsophalangeal joint of digit $\mathrm{V}$. The lateral $\mathrm{m}$. dorsometatarsalis proximalis digiti $\mathrm{V}$ is well developed, originating proximally on the dorsal and distally on the dorsolateral surface of metatarsal $\mathrm{V}$ and inserting on the lateral portion of the proximal interphalangeal joint of digit $\mathrm{V}$.

Presumed function: To extend the phalanges and assist in detaching the adhesive discs.

MM. DORSOMETATARSALES DisTALES: The lateral muscles of this group are present in the five digits; a medial muscle is confined to digit V. The lateral $\mathrm{m}$. dorsometatarsalis hallucis distalis has a fleshy origin on the laterodistal end of metatarsal I; its fibers reach around the proximal $3 / 4$ of the basal phalanx and insert via a tendon on the interphalangeal joint/distal phalanx of digit I. The lateral $\mathrm{m}$. dorsometatarsalis distalis digiti II has a fleshy origin from the laterodistal end of metatarsal II; its fibers reach around the proximal 3/4 of the basal phalanx and insert via a tendon on the interphalangeal joint/distal phalanx of digit II. The lateral $\mathrm{m}$. dorsometatarsalis distalis digiti III has a fleshy origin from the laterodistal end of metatarsal III; its fibers extend along the proximal $1 / 5$ of the second phalanx and insert via a tendon on the distal interphalangeal joint/distal phalanx of digit III. The lateral $\mathrm{m}$. dorsometatarsalis distalis digiti IV has a fleshy origin from the laterodistal end of metatarsal IV; its fibers reach the distal end of the basal phalanx and insert via a tendon on the distal interphalangeal joint/distal phalanx of digit IV.

The medial $\mathrm{m}$. dorsometatarsalis distalis digiti $\mathrm{V}$ has a fleshy origin on the mediodistal end of metatarsal V; its fibers reach around the proximal 3/4 of the second phalanx and insert via a tendon on the distal interphalangeal joint/distal phalanx of digit V. The lateral $\mathrm{m}$. dorsometatarsalis distalis digiti $V$ has a fleshy origin from the laterodistal end of metatarsal V (the fibers arise more proximately on the metatarsal than other $\mathrm{mm}$. dorsometatarsales distales). The fibers almost reach the distal end of the basal phalanx and insert via a tendon on the distal interphalangeal joint/distal phalanx of digit $\mathrm{V}$.
Presumed function: To extend the digits and detach the adhesive disc.

Comments: There are up to six muscles of this group in some of our studied species, as reported for Triprion petasatus. A seventh muscle (the medial muscle of digit IV) was reported by Burton (2004) for three hylids (Hyla japonica, Litoria dentata, and L. modica) we did not study.

MM. ABDUCTORES BREVES DORSALES: Only the $\mathrm{m}$. abductor brevis dorsalis hallucis is present. It is a single muscle that arises from a fleshy origin on the prehallux and element $\mathrm{Y}$ and has a fleshy insertion on the medial and proximal surfaces of metatarsal I.

Presumed function: To abduct digit I through its metatarsal.

Comments: A muscle of this group is present in digits II and III of some species (see chars. 19-20).

M. ABDUCTOR PROPRIUS DIGITI IV: This muscle has a fleshy origin from the proximal and medial surfaces of metatarsal $\mathrm{V}$; it has a fleshy insertion covering slightly more than the proximal half of the lateral surface of metatarsal IV.

Presumed function: To abduct metatarsal IV.

Comments: As stated by Burton (2004: 213), although the $\mathrm{m}$. abductor proprius digiti IV is considered a plantar muscle (e.g., Gaupp, 1896), it is more easily approached from the dorsal side, leading us to describe and figure it with the dorsal muscles.

Dorsal Surface of the Foot: Muscles Unassigned to Layer (pl. 5A, D)

M. TARSALIS ANTICUS: This muscle arises from the distal end of the tibiofibula and has a fleshy insertion on the dorsal surface of the tibiale.

Presumed function: Dorsal flexor and supinator of the tibiale and fibulare (Gaupp, 1896: 216).

M. ABDUCTOR DIGITI MINIMI: This large muscle lies lateral to the $\mathrm{m}$. extensor digitorum longus. It has a fleshy origin on almost the entire dorsolateral surface of the fibulare, lateral to the origin of the $\mathrm{mm}$. extensores breves superficiales. It inserts via a short tendon on the dorsal and proximal surfaces of metatarsal V, proximal to the ori- 
gin of the lateral $\mathrm{m}$. dorsometatarsalis proximalis digiti $\mathrm{V}$ and $\mathrm{m}$. abductor proprius digiti IV.

Presumed function: To abduct metatarsal V, which might be relevant during the propelling kick in swimming (see Gaupp, 1896: 222).

\section{Character Evolution}

Below we describe 20 characters from the hand and foot musculature of anurans. Plate 1 shows the major clades for which these characters optimize as synapomorphies in a reduced family tree following Jetz and Pyron (2018). The data matrix and ancestral character state reconstructions (ACSRs) are shown in appendices 4 and 5 , respectively.

\section{Palmar Surface of the Hand}

Character 1: M. flexor indicis superficialis proprius: (0) absent (pl. 6A); (1) present (pls. 2A, 6B-D).

Definition: The $\mathrm{m}$. flexor indicis superficialis proprius originates on the medial margin of distal carpal 3-4-5 (or, if these distal carpals are not fused, from one or more of the medialmost carpals) and inserts via a long, sturdy tendon on the palmar surface of the distal phalanx of digit II (Burton, 1998a; this study). This muscle must not be confounded with the tendo superficialis indicis, first defined as such here, which extends from the flexor plate to the same point of the distal phalanx as the $\mathrm{m}$. flexor indicis superficialis proprius. When the tendo superficialis indicis co-occurs with the $\mathrm{m}$. flexor indicis superficialis proprius, both elements converge to form a common tendon of insertion on the distal phalanx (Burton, 1983; pl. 6B-C), as also occurs with the m. caput profundum digiti III and tendo superficialis digiti III (pls. 2A, 6B-D).

ACSR and taxonomic distribution: The presence of this muscle optimizes as a synapomorphy of Acosmanura; no instances of homoplasy are known.

Character 2: M. lumbricalis longus digiti III: (0) absent (pls. 2A, 6A-B, D); (1) present (pl. $6 \mathrm{C})$.
Definition: This muscle originates on the dorsal surface of the tendo superficialis digiti III as a pair of slender slips and inserts on the basal phalanx on both sides of the tendo superficialis. It was first described by Burton (1998a: 66, 1998b: 5), but we observed the following deviations from his description: (1) the origin can extend proximad to include the distal end of the flexor plate; (2) the medial and lateral slips can be completely or partially undifferentiated, with both being only distally differentiated, or represented only by the medial slip (lateral slip absent or undifferentiated); and (3) the m. lumbricalis longus digiti III can also insert on the metacarpophalangeal joint (as observed in the lateral tendon of insertion in pl. 6C).

ACSR and taxonomic distribution: The presence of the $\mathrm{m}$. lumbricalis longus digiti III optimizes as a synapomorphy of Heleophrynidae. It is also a synapomorphy of Calyptocephalellidae, since, besides being present in Calyptocephalella, it was also reported by Burton (1998b) for two species of Telmatobufo (exemplars of this taxon not included in the present study). Burton (1998b) also reported the $\mathrm{m}$. lumbricalis longus digiti III in Odontobatrachus (Odontobatrachidae; as Petropedetes), Petropedetes (Petropedetidae), Telmatobius brachydactylus, and T. macrostomus (Telmatobiidae; as Lynchophrys and Batrachophrynus, respectively). Although the taxonomic distribution of character 2 of Burton (1998b) did not list Alytes (Alytidae; A. muletensis and A. obstetricans were his exemplars) as having $\mathrm{m}$. lumbricalis longus digiti III, in the text they are described as possessing it. We studied two specimens of $A$. obstetricans and recorded the absence of the $\mathrm{m}$. lumbricalis longus digiti III. Also, we observed the m. lumbricalis longus digiti III in Ascaphus truei and Bombina variegata (contra Burton, 1998b). See Barrionuevo (2017) for discussion and clarification of its occurrence within Telmatobiidae.

Character 3: M. lumbricalis brevis indicis, origin from flexor plate: (0) absent; (1) present.

Definition: The $\mathrm{m}$. lumbricalis brevis indicis originates on the medial side of distal carpal 3-4-5 (or, if the distal carpals are not fused, from one or 
more of the medialmost distal carpals) and/or the flexor plate and inserts on the metacarpophalangeal joint of digit II via either a short tendon or fleshy insertion (Burton, 1998a; this study).

ACSR and taxonomic distribution: The absence of an origin of the $\mathrm{m}$, lumbricalis brevis indicis from the flexor plate optimizes as a synapomorphy of Xenoanura + Acosmanura. Within this clade, the origin from the flexor plate subsequently reevolved in Calyptocephalella gayi (Calyptocephalellidae), Hadromophryne natalensis (see char. 4), and Heleophryne purcelli (Heleophrynidae). Within Xenoanura, the origin from the flexor plate is present in Hymenochirini (i.e., Hymenochirus + Pseudhymenochirus) and Xenopus (excluding $X$. epitropicalis). The m. lumbricalis brevis indicis is absent in Geocrinia victoriana (Myobatrachidae).

CHARACTER 4: M. lumbricalis brevis indicis, origin from distal carpals: (0) absent; (1) present (pl. 6D).

Definition: See character 3.

ACSR and taxonomic distribution: The presence of an origin of the $\mathrm{m}$. lumbricalis brevis indicis from the distal carpals optimizes as a synapomorphy of Xenoanura + Acosmanura, while its absence is a synapomorphy of (1) Hymenochirini and (2) an internal clade of Xenopus. Barbourula busuangensis and Bombina bombina (Bombinatoridae) lack the origin from the distal carpals, as does Hadromophryne natalensis (Heleophrynidae) as well. This is partially concordant with the description of Burton (1998a: 66) for $H$. natalensis, although he described an origin from an element of the prepollex, whereas the origin is exclusively from the flexor plate in the specimen we examined. Presumably (based on the fact that he did not describe variation in this muscle), the m. lumbricalis brevis indicis originates on the distal carpals in all the species of "Leptodactylidae" (sensu lato) examined by Burton (1998a).

ChARACTER 5: M. lumbricalis brevis digiti III, slip originating from flexor plate and/or tendo superficialis digiti III: (0) absent; (1) present (pl. $6 \mathrm{~A}, \mathrm{C})$.
Definition: This slip originates from the flexor plate and/or adjacent tendo superficialis digiti III and inserts on the metacarpophalangeal joint or proximally on the basal phalanx of digit III (Burton, 1996, 1998b; this study). We consider this slip to be distinct from the slip that originates from the distal carpals (treated in char. 6), as further discussed and justified under the section "Calyptocephalellidae" in the Discussion. Note that Burton (1998b: char. 3) scored both slips (from distal carpals and from the flexor plate/tendo superficialis) within the same transformation series in a multistate character. Additionally, we consider the muscle referred to as the "medial slip to the $\mathrm{m}$. lumbricalis brevis digiti III" in character 9 of Burton (1998a) to be homologous with the slip of the $\mathrm{m}$. lumbricalis brevis digiti III from the flexor plate and/or adjacent tendo superficialis digiti III.

ACSR and taxonomic distribution: The optimization of this character is ambiguous for the major clades. The basal ambiguity is a consequence of its presence in (1) Ascaphidae, Leiopelmatidae, Costata, and Xenoanura, (2) Heleophrynidae, and (3) Australobatrachia. See appendices 4 and 5 for complete taxonomic distribution. Its presence is polymorphic in Kassina senegalensis (Hyperoliidae; Burton, 1998b; 6) but is present in the single specimen examined by us.

Character 6: M. lumbricalis brevis digiti III, slip originating from the distal carpals: (0) absent; (1) present (pl. 2B, C).

Definition: This slip of the $\mathrm{m}$. lumbricalis brevis digiti III has a tendinous origin on the distal carpals and inserts on the metacarpophalangeal joint or proximally on the basal phalanx of digit III (Burton, 1996, 1998b; this study).

ACSR and taxonomic distribution: The absence of this slip optimizes as a synapomorphy of Myobatrachoidea (Myobatrachidae + Limnodynastidae); it is also absent in Hadromophryne natalensis and Heleophryne purcelli (the optimization is ambiguous in this part of the tree). Within Limnodynastidae, this slip of the m. lumbricalis brevis digiti III is present in Lechriodus melanopyga and Platyplectrum ornatum. 
Character 7: Medial m. lumbricalis brevis digiti IV, supplementary medial slip originating from the distal carpals: (0) absent; (1) present (pl. 7).

Definition: This slip, first reported here, is tentatively considered a supplementary slip of the medial $\mathrm{m}$. lumbricalis brevis digiti IV in light of its position and common insertion. This supplementary slip shares a common origin with the $\mathrm{m}$. lumbricalis brevis digiti III on the distal carpals and converges distally with the insertion of the medial $\mathrm{m}$. lumbricalis brevis digiti IV on the metacarpophalangeal joint of digit IV.

The medial $\mathrm{m}$. lumbricalis brevis digiti IV originates on the flexor plate (or the tendo superficialis digiti IV in species with a reduced flexor plate) in most species but originates on the distal carpals in a few species, such as in some microhylids (Burton, 1983, 1986; also observed by us is Chaperina fusca, Elachistocleis bicolor, Kalophrynus sinensis, and Microhyla heymonsi) and cacosternines (Cacosternum capense and Microbatrachella capensis). The question that arises here is whether this supplementary slip is homologous with the medial $\mathrm{m}$. lumbricalis brevis digiti IV originating on the carpals, based on the fact that both originate on the carpals.

Nevertheless, we consider the medial m. lumbricalis brevis digiti IV originating on the carpals to be homologous with the medial $\mathrm{m}$. lumbricalis brevis digiti IV originating on the flexor plate (and not to the supplementary medial slip as defined here); this assertion is based on two points. First, the supplementary slip is located more medially, whereas the medial m. lumbricalis brevis digiti IV from the distal carpals is located in the same topographical region as the medial $\mathrm{m}$. lumbricalis brevis from the flexor plate. Second, the medial $\mathrm{m}$. lumbricalis brevis digiti IV originating on the flexor plate does not co-occur with the medial $\mathrm{m}$. lumbricalis brevis digiti IV originating on the distal carpals (Burton, 1986; this study), suggesting their homology by way of the conjunction test (Patterson, 1982).

ACSR and taxonomic distribution: The presence of this supplementary slip optimizes as a synapomorphy of Megophryidae, with no known instances of homoplasy.

CHARACTER 8: M. flexor minimus indicis: (0) absent; (1) present (pls. 8C, 9A).

Definition: This muscle has a fleshy origin from the ventral surface of metacarpal II and inserts via a tendon on the basal phalanx, adjacent to the insertion of the $\mathrm{m}$. contrahentis indicis. This muscle was first described by Ribbing (1911) in Pipa cf. pipa and corroborated in our specimen of Pipa pipa. See Homology and terminology: a reappraisal, below, for a discussion of its identity.

ACSR and taxonomic distribution: Present in Ascaphus (Ascaphidae) and Leiopelma (Leiopelmatidae); its polarity in Ascaphidae + Leiopelmatidae is contingent on the condition in Caudata (see Discussion). Its presence is a synapomorphy of Heleophryne (Heleophrynidae) and, possibly, Sooglossus (Sooglossidae) or a more inclusive clade (state unknown in the sooglossid Sechellophryne and Nasikabatrachidae). This muscle is also present in Amazophrynella minuta (Bufonidae), Chaperina fusca (Microhylidae), and Pipa pipa (Pipidae).

Character 9: M. flexor minimus digiti IV, position of the origin relative to the $\mathrm{m}$. intermetacarpalis II: (0) $\mathrm{m}$. flexor minimus digiti IV ventral to m. intermetacarpalis II (pls. 8C, 9A, 9C-D, 10A); (1) m. flexor minimus digiti IV dorsal to $\mathrm{m}$. intermetacarpalis II (pls. 2D, 10B); (2) $\mathrm{m}$. flexor minimus digiti IV ventral and dorsal to m. intermetacarpalis II (pl. 8B).

Definition: The $\mathrm{m}$. flexor minimus digiti IV has a fleshy origin from the mediopalmar surface of metacarpal IV, dorsally and/or ventrally to the $\mathrm{m}$. intermetacarpalis II, and it inserts via tendon or fleshy on the basal phalanx of digit IV. This character was defined by Burton (1998a: char. 11, 1998b). In the absence of developmental or other evidence to establish nested homology, we treat this character as nonadditive (unordered).

ACSR and taxonomic distribution: A dorsal origin (state 1) optimizes as synapomorphies of Hymenochirini and Nobleobatrachia, while a ventral and dorsal origin (state 2 ) is a 
synapomorphy of Bombinatoridae. The taxonomic distribution of this character is complex and entails several instances of homoplasy (see appendices 4 and 5). Burton (1998b) examined this character for a broad sample of anurans. The major conflict between Burton's report and our observations is that he scored the $\mathrm{m}$. flexor minimus digiti IV as having a ventral origin in Bombinatoridae and a dorsal origin in Pipidae (Burton, 1998b: table 1), whereas we found the origin to be both ventral and dorsal in Bombinatoridae and ventral in some species of Pipidae. See Discussion under the sections of Bombinatoridae and Pipidae for possible explanations of the disagreement.

Diogo and Ziermann (2014: 93) did not observe the $\mathrm{mm}$. flexores digitorum minimi in their study of the development of the forelimb and hind limb musculature of Eleutherodactylus coqui, stating that these muscles might be absent or very deep and/or deeply blended with other hand muscles. In contrast, we observed the typical set of $\mathrm{mm}$. flexores digitorum $\mathrm{min}$ imi (on digits III-V) in E. coqui (pl. 10B), as did Burton (1998a, 1998b). See Discussion for the interpretation of Diogo and Ziermann (2014) of these muscles as part of their $\mathrm{mm}$. flexores breves profundi.

CharaCter 10: M. contrahentis caput longus distalis: (0) absent (pls. 9D, 10A); (1) present (pls. 2D, 9A-C, 10B).

Definition: As described by Burton (1998a), the $\mathrm{m}$. contrahentis caput longus distalis has a fleshy origin on the medial surface of the ulnare and a fleshy insertion on the proximal surface of distal carpal 3-4-5 (in species with different carpal morphology the insertion is on distal carpal 4-5, distal carpal 4-5 and distal carpal 3, distal carpal 4, or distal carpal 4 and distal carpal 5; this study).

ACSR and taxonomic distribution: The absence of the $\mathrm{m}$. contrahentis caput longus distalis optimizes as a synapomorphy of Anomocoela (Scaphiopodidae + Pelodytidae + Pelobatidae + Megophryidae) with no instances of homoplasy.
Dorsal Surface of the Hand

Character 11: M. extensor brevis medius digiti III, slip from the mediodistal end of radioulna: (0) absent; (1) present (pl. 13).

Definition: This slip from the radioulna is not homologous with the slips from the radiale and element $Y$, since all three slips are present in some taxa (i.e., their homology is refuted by the conjunction test; Patterson, 1982). It is characterized by a fleshy or tendinous origin from the dorsum of the tendon of insertion of the $m$. extensor carpi radialis at the level of the mediodistal end of the radioulna. It inserts via a flat tendon, partially on the medial region of the metacarpophalangeal joint and partially on the medial region of the interphalangeal joint of digit III.

ACSR and taxonomic distribution: The presence of this slip from the radioulna optimizes as a synapomorphy of Pelodytidae + Pelobatidae + Megophryidae, with no known instances of homoplasy. We also observed a slip of the $\mathrm{m}$. extensor brevis medius digiti III from the radioulna in Craugastor fitzingeri (Craugastoridae); however, its origin is located more medially on the radioulna, does not originate from the tendon of insertion of the $\mathrm{m}$. extensor carpi radialis, and converges proximally with the slip from the radiale to form a single muscular body. Note that even if the condition in Craugastor is considered a primary homolog of the slip in Pelodytidae + Pelobatidae + Megophryidae, the cladistic distance between the taxa is great enough that its optimization as a synapomorphy of this major clade is not affected.

Character 12: $\mathrm{M}$. extensor brevis medius digiti IV, origin from the radiale: (0) absent; (1) present (pl. 13).

Definition: The $\mathrm{m}$. extensor brevis medius digiti IV has a broad and continuous origin from the radiale and/or element Y (element Y-distal carpal 2 if these elements are fused) or independent origins from the radiale and element $Y$, with a distal convergence into a single muscle. The insertion is variable, on the distal end of metacarpal IV, the metacarpophalangeal joint, proximal 
interphalangeal joint, and/or distal interphalangeal joint/distal phalanx; this last condition occurs when the $\mathrm{m}$. extensor brevis medius digiti IV converges into a common insertion with the medial $\mathrm{m}$. dorsometacarpalis proximalis digiti IV (Burton, 1998a; this study). We scored each origin or head as a separate character and present the data for the origin from the radiale, which is synapomorphic for a major clade.

ACSR and taxonomic distribution: The presence of an origin on the radiale optimizes as a synapomorphy of Acosmanura. Some additional independent reversions occur within Acosmanura (see appendices 4 and 5). Among non-acosmanuran species, the origin from the radiale is only present in Xenopus laevis. The m. extensor brevis medius digiti IV is absent in Acris crepitans, Ranoidea eucnemis (Hylidae; also reported as absent for R. eucnemis by Burton, 1996), Alytes obstetricans (Alytidae), Ascaphus truei (Ascaphidae), and Pseudhymenochirus merlini (Pipidae). Pipa parva has a particular carpal morphology (Fabrezi, 1992), in which the radiale, ulnare, and distal carpal 5 are fused. In this species, the origin of the $\mathrm{m}$. extensor brevis medius digiti IV on the radiale was scored as absent since it was restricted only to the fused element $\mathrm{Y}$ with the distal carpal 2-3. Hoyos and Salgar (2016: 146147) described an origin from the distal carpal 3-4-5 in Dendropsophus luddeckei (as D. labialis; Hylidae), previously unreported in anurans; nevertheless, the single specimen of $D$. luddeckei we examined has the typical origin from element $Y$ and the radiale.

ChARACTER 13: Medial $\mathrm{m}$. dorsometacarpalis proximalis digiti IV, slip from metacarpal III: (0) absent; (1) present (pl. 3D).

Definition: The $\mathrm{mm}$. dorsometacarpales proximales of digit IV consist of a medial and a lateral muscle, each of which is usually composed of two slips (see Burton, 1998a: fig. 2D). The medial muscle has one slip that originates from the proximolateral surface of metacarpal III and the other from the dorsomedial surface of metacarpal IV; they usually converge into a common tendon that inserts on the distal interpha- langeal joint/distal phalanx and, in some species, also on the proximal interphalangeal joint of digit IV. The lateral muscle has one slip that originates from the base of metacarpal $\mathrm{V}$ and another from the dorsolateral side of metacarpal IV; they usually converge into a single tendon to insert on the proximal and/or distal interphalangeal joint/ distal phalanx of digit IV (Burton, 1998a; this study). The slip from metacarpal III of the medial $\mathrm{m}$. dorsometacarpalis proximalis digiti IV is absent in some species.

ACSR and taxonomic distribution: The absence of the slip from metacarpal III of the medial $\mathrm{m}$. dorsometacarpalis proximalis digiti IV is homoplastic, optimizing as synapomorphies of Bombinatoridae and Pipidae. This slip is also absent in Leptobrachium lumadorum (Megophryidae), Phrynomantis bifasciatus (Microhylidae), Crinia signifera, and Pseudophryne coriacea (both Myobatrachidae).

Plantar Surface of the Foot

Character 14: M. lumbricalis brevis hallucis: (0) absent; (1) present (pl. 4B).

Definition: The $\mathrm{m}$. lumbricalis brevis hallucis has either a broad, fleshy origin or originates via a discrete tendon on the dorsomedial and distal margins of the aponeurosis plantaris, just lateral to the prehallux. Its lateral margin is usually adjacent to the tendo superficialis hallucis and it inserts proximally on the basal phalanx or on the metatarsophalangeal joint of digit I (Dunlap, 1960; Burton, 2004; this study). This muscle is absent in the studied species of Xenoanura.

ACSR and taxonomic distribution: The absence of the $\mathrm{m}$. lumbricalis brevis hallucis optimizes as a nonhomoplastic synapomorphy of Xenoanura. This muscle is also absent in Pipa pipa (Pipidae) according to Dunlap (1960: 35).

Character 15: Lateral $\mathrm{m}$. lumbricalis brevis digiti IV, presence of a supplementary origin from the proximal end of metatarsal IV: (0) absent; (1) present.

Definition: The origin of the lateral m. lumbricalis brevis digiti IV can be fleshy or tendinous and from one or more of the following points: (1) 
the plantar cartilage at the base of metatarsal V, (2) the distal end of the aponeurosis plantaris, (3) the tendon of insertion of the $\mathrm{mm}$. flexores breves superficiales, and (4) the dorsal surface of the tendo superficialis digiti IV. It inserts proximally on the ventrolateral margin of metatarsophalangeal joint or also on the proximal interphalangeal joint of digit IV (Dunlap, 1960: 36; Burton, 2004; this study). We report for the first time a supplementary tendinous origin from the proximal and ventral end of metatarsal IV, present in all examined scaphiopodids. In this family, the lateral $\mathrm{m}$. lumbricalis brevis digiti IV also shares a common origin with the $\mathrm{m}$. lumbricalis longus digiti $\mathrm{V}$ from the single tendon of insertion of the $\mathrm{mm}$. flexores breves superficiales.

Although we examined all species of Scaphiopodidae and observed the occurrence of both origins (from metatarsal IV and mm. flexores breves superficiales) in all of them, Dunlap (1960: 36) did not describe an origin from metatarsal IV for Spea hammondii (the only scaphiopodid he examined). See Discussion for possible explanations of this disagreement.

ACSR and taxonomic distribution: The presence of this supplementary origin is a nonhomoplastic synapomorphy of Scaphiopodidae and was observed in all species of the family we studied.

Character 16: M. contrahentis pedis digiti V: (0) absent; (1) present (pl. 11A).

Definition: The $\mathrm{m}$. contrahentis pedis digiti $\mathrm{V}$ originates from the distal tarsals and inserts on metatarsal $\mathrm{V}$ and/or basal phalanx of digit $\mathrm{V}$ (Dunlap, 1960: 39-40; this study).

ACSR and taxonomic distribution: The absence of the $\mathrm{m}$. contrahentis pedis digiti $\mathrm{V}$ optimizes as a synapomorphy of Neobatrachia with no known instances of homoplasy.

\section{Dorsal Surface of the Foot}

Character 17: M. extensor brevis superficialis digiti III: (0) absent; (1) present (pls. 5B, 12A).

Definition: The $\mathrm{m}$. extensor brevis superficialis digiti III has a fleshy or tendinous origin from the fibulare. It inserts at one or more of the following points: fleshy or tendinous insertion on the dorsal surface of metatarsal III, tendinous insertion on the metatarsophalangeal joint, and/ or tendinous insertion on the proximal interphalangeal joint of digit III (Dunlap, 1960; Burton, 2004; this study).

ACSR and taxonomic distribution: The absence of the $\mathrm{m}$. extensor brevis superficialis digiti III is a synapomorphy of Sooglossidae (or Sooglossoidea, if also absent in Nasikabatrachus and Sechellophryne). It is absent in Leptodactylus fuscus and L. savagei (reported as L. pentadactylus; Burton, 2004) and polymorphic in $L$. albilabris (Leptodactylidae; Dunlap, 1960: 49). Burton (2004: 225, his char. 51) also mentioned the absence of the $m$. extensor brevis superficialis digiti III in Ceratophrys cornuta (Ceratophryidae); however, in his list of specimens examined the specimen is reported as C. ornata with voucher number USNM 115606, whose locality data correspond to the distribution of C. aurita. As such, the absence of the m. extensor brevis superficialis digiti III should be attributed to C. aurita instead of C. cornuta or C. ornata.

Character 18: M. extensor brevis medius digiti IV: (0) absent; (1) present (pl. 12B).

Definition: This muscle originates with the other $\mathrm{mm}$. extensores breves medii from the distal end of the fused tibiale and fibulare and inserts either with the medial $\mathrm{m}$. extensor brevis superficialis digiti IV or independently on the metatarsophalangeal joint of digit IV (Dunlap, 1960; this study). See appendix 3 for discussion of confusion across the literature between the $\mathrm{m}$. extensor brevis medius digiti IV and the $\mathrm{m}$. extensor brevis superficialis digiti IV.

ACSR and taxonomic distribution: The presence of this muscle optimizes as synapomorphy of Alytidae (character state unknown in Latonia). Ascaphus truei (Ascaphidae) is polymorphic (Dunlap, 1960; this study). Dunlap (1960) did not report this muscle in his single specimen of Discoglossus pictus (Alytidae), but it is present in the specimen we examined (see Discussion for possible explanations of the disagreement). This muscle was also reported in Heleioporus austra- 
liacus (Limnodynastidae) and "Eleutherodactylus tubolus" (nomen nudum; no voucher specimen or locality reported) by Dunlap (1960: 55).

Character 19: M. abductor brevis dorsalis digiti II: (0) absent; (1) present (pl. 14).

Definition: This muscle, first reported here, originates in common with or contiguous to the medial $\mathrm{m}$. dorsometatarsalis proximalis digiti II on the proximolateral margin of metatarsal I and has a fleshy insertion on the medial side of metatarsal II (see Discussion).

ACSR and taxonomic distribution: The presence of the $\mathrm{m}$. abductor brevis dorsalis digiti II optimizes as synapomorphic for Laurentobatrachia and Leptobrachiinae (Megophryidae; with a reversion in Scutiger mammatus). Also, it is a synapomorphy of Sooglossidae (or Sooglossoidea, if present in Nasikabatrachus and Sechellophryne). This muscle is also present in Neobatrachus aquilonius (Limnodynastidae).

Character 20: M. abductor brevis dorsalis digiti III: (0) absent; (1) present (pl. 14).

Definition: This muscle, first reported here, originates in common with or contiguous to the medial $\mathrm{m}$. dorsometatarsalis proximalis digiti III on the proximolateral margin of metatarsal II and has a fleshy insertion on the medial side of metatarsal III.

ACSR and taxonomic distribution: The presence of the $\mathrm{m}$. abductor brevis dorsalis digiti III optimizes as a synapomorphy of Laurentobatrachia, and is also a synapomorphy of Sooglossidae (or Sooglossoidea, if present in Nasikabatrachus and Sechellophryne). This muscle is also present in Leptobrachium lumadorum and Oreolalax omeimontis (Megophryidae), and Neobatrachus aquilonius (Limnodynastidae).

\section{DISCUSSION}

\section{Systematic Implications OF THE INFERRED SYNAPOMORPHIES}

Ascaphidae + Leiopelmatidae

Frost et al. (2006) recovered some phenotypic synapomorphies for Ascaphidae + Leiopelmatidae (as Leiopelmatidae) and discussed others from the literature. Within the context of the sister-group relationship of Ascaphus and Leiopelma, Frost et al. (2006) reinterpreted the characters suggested by Ford and Cannatella (1993) as evidence uniting Leiopelma with all remaining frogs (to the exclusion of Ascaphus). Pereyra et al. (2016) further discussed the loss of the columella as a synapomorphy of this clade in the context of broad taxon sampling and considering the condition in relevant fossil taxa.

Ascaphus and Leiopelma have a muscle identified as the m. flexor minimus indicis (char. 8; see the section "Homology and terminology: a reappraisal" for discussion of its identity). The current consensus is that the radialmost (preaxial) finger of Anura is homologous with digit II of five-fingered tetrapods (implying that anurans lost digit I; see Fabrezi and Alberch, 1996; Young et al., 2011), while the radialmost finger of caudates has been interpreted as digit I of five-fingered tetrapods (e.g., Alberch and Gale, 1985; Diogo and Abdala 2010). While these homology assessments require further corroboration, below we compare the radialmost digit of Anura with digit II of Caudata.

Studies of the hand musculature of Caudata are scarce, with information restricted to four species of Ambystoma (Ambystomatidae; Perrin, 1899; Ribbing, 1907; Diogo and Tanaka, 2012), Andrias japonicus (Cryptobranchidae; Humphry, 1871), Bolitoglossa adspersa (Plethodontidae; Restrepo and Hoyos, 1998), Cryptobranchus alleganiensis (Cryptobranchidae; Ribbing, 1907), Necturus maculosus (Proteidae; Ribbing, 1907), Pleurodeles waltl (Salamandridae; Perrin, 1899), Salamandra salamandra (Salamandridae; Perrin, 1899; Ribbing, 1907; Francis, 1934), Taricha torosa (Salamandridae; Walthall and Ashley-Ross, 2006), and Triturus cristatus (Salamandridae; Ribbing, 1907). There is no report of $\mathrm{mm}$. flexores digitorum minimi in $T a$. torosa, while the presence of these muscles are restricted to digits I and IV in some Ambystoma, and digits II-IV in Salamandra. For the remaining species, (except Bolitoglossa, which is unknown) the $\mathrm{mm}$. flexores digitorum minimi are present in the four digits. Considering the available evidence, 
and the above-mentioned caveats, the presence of the m. flexor minimus indicis in Ascaphidae + Leiopelmatidae is possibly plesiomorphic for this clade. Nevertheless, analysis of a broader sample of caudates is required, including hynobiids (relevant for establishing if the $\mathrm{m}$. flexor minimus of digit II is plesiomorphically present in Caudata). Similarly, if new evidence indicates that the musculature of the radialmost digits of Anura and Caudata are homologous, the plesiomorphic presence of the $\mathrm{m}$. flexor minimus of the radialmost finger of caudates would also be particularly relevant.

Bombinatoridae

Ford and Cannatella (1993) listed two synapomorphies for this group: an expanded flange of the quadratojugal and endochondral ossifications in the hyoid plate. Haas (2003) and Frost et al. (2006) recovered the presence of the $\mathrm{m}$. mandibulolabialis superior in larvae as an additional synapomorphy; however, only species of Bombina were scored for this character (larvae of Barbourula are unknown).

Here we add two more synapomorphies for this group: (1) ventral and dorsal origin of the $\mathrm{m}$. flexor minimus digiti IV with respect to the m. intermetacarpalis II (char. 9.2) and (2) absence of the slip from metacarpal III of the medial $\mathrm{m}$. dorsometacarpalis proximalis digiti IV (char. 13.0). Burton (1998b) examined the first character in a taxonomically broad sample. Importantly, both our study and Burton's scored this character in Bombina orientalis, B. variegata, and Barbourula busuangensis, and we also studied it in Bombina bombina (note that the data on B. bombina provided by Burton, $1998 \mathrm{~b}$, are restricted to the superficial muscles of the third digit and do not include this character; see his appendix 1). Curiously, Burton (1998b) described only a ventral origin (our state 0 ) of the $\mathrm{m}$. flexor minimus digiti IV and not dual ventral and dorsal origins (our state 2).

We propose two complementary explanations for the discrepancies between Burton's (1998b) scoring of this character and our own. First, the analyzed species of Bombina have an m. contrahentis digiti IV (see pl. 8A), which is absent in
Barbourula busuangensis (pl. 8B). The m. contrahentis digiti IV is ventral (superficial) to the $\mathrm{m}$. flexor minimus digiti IV, and both converge distally on the insertion point on the basal phalanx. Second, the ventral portion of the $\mathrm{m}$. flexor minimus digiti IV can be well developed, concealing the dorsal portion due to its overlying position (see pl. 8B for partial concealment and pl. 9B for complete concealment). In this case, the combination of the $\mathrm{m}$. contrahentis digiti IV and a well-developed ventral portion of the $\mathrm{m}$. flexor minimus digiti IV might hide the dorsal portion, which could go unnoticed in case of, for example, partial or incomplete dissection of the more ventral/superficial muscles.

At this point, it is relevant to clarify that a similar disagreement arises regarding the relationships between the $\mathrm{m}$. intermetacarpalis I and the m. flexor minimus digiti III. Burton (1998b) described a ventral $\mathrm{m}$. flexor minimus digiti III with respect to the $\mathrm{m}$. intermetacarpalis $\mathrm{I}$ in Bombinatoridae. As depicted in plates $8 \mathrm{~A}-\mathrm{B}$ and $9 \mathrm{~B}$, we also observed a dorsal origin of the $\mathrm{m}$. flexor minimus digiti III with respect to the $\mathrm{m}$. intermetacarpalis I in all examined bombinatorids except $B$. bombina (not studied by Burton for this character), which has only a dorsal origin. Although additional studies are required to evaluate intraspecific variation, the same explanations offered above for the incongruence between Burton's (1998b) description and our observations apply here.

Exemplars of the other main clade of Bombina (such as B. maxima and B. microdeladigitora; see Pabijan et al., 2013; Pyron, 2014; Jetz and Pyron, 2018) were not included by either Burton (1998b) or us. Exemplars of this clade are required to test the hypothesized synapomorphies of Bombinatoridae.

Alytidae

The reanalysis of Haas's characters (2003) combined with the molecular data in Frost et al. (2006) recovered two larval character states as synapomorphies of this family: (1) admandibular cartilage present (see Haas, 2003: char. 95.1) and 
(2) processus postcondylaris of the ceratohyal present (see Haas, 2003: character 118.1). Here, we propose an additional synapomorphy: the presence of the foot $\mathrm{m}$. extensor brevis medius digiti IV (char. 18.1; pl. 12B).

Dunlap (1960: 55) did not report the $\mathrm{m}$. extensor brevis medius digiti IV for the single specimen of Discoglossus pictus he examined; however, it is present in the two species of Discoglossus examined here (D. galganoi and $D$. pictus), although it is partially fused to the medial $\mathrm{m}$. dorsometatarsalis proximalis digiti IV (which originates from the distal end of the tibiale). The disagreement regarding the presence of the $\mathrm{m}$. extensor brevis medius digiti IV in $D$. pictus might be due to either the partial fusion with the $\mathrm{m}$. dorsometatarsalis proximalis, leading Dunlap (1960) to overlook it, or polymorphism; further sampling is required to resolve the issue. Occurrence of this muscle is polymorphic in Ascaphus truei, in which Dunlap (1960: 55) described it as a very small muscle that is absent in some specimens; we also found it to be absent in one of the two specimens examined.

\section{Xenoanura + Acosmanura}

Previously proposed synapomorphies for this clade are (1) fusion of the facial and trigeminal ganglia; (2) sinistral spiracle in larvae; (3) absence of free ribs in adults (but see Haas, 2003: 75); (4) torsion of carpal elements; and (5) presence of a vocal sac (Sokol, 1975, 1977; Ford and Cannatella, 1993). Here we add two phenotypic synapomorphies involving the origin of the $\mathrm{m}$. lumbricalis brevis indicis: the absence of an origin from the flexor plate (char. 3.0) and the presence of an origin from the carpals (char. 4.1).

It must be noted that the pattern of the ancestral character state reconstruction in Xenoanura + Acosmanura is compatible with a simple switch in the origin of the $\mathrm{m}$. lumbricalis brevis indicis from the flexor plate to the distal carpals, since both characters change at the same node. However, the occurrence of both origins (from the carpals and flexor plate) in several species pro- vides evidence for the historical independence of the two transformation series generally via the conjunction test (Patterson, 1982). In any case, the issue must be reevaluated in future studies as more data become available. Although we cannot advance a specific hypothesis at this time, the simultaneous changes in both characters suggest a possible relation with carpal torsion.

\section{Xenoanura}

Several characters have been proposed as synapomorphies for this group (see Ford and Cannatella, 1993; Haas, 2003; Frost et al., 2006). Here we propose the first synapomorphy in adult musculature: absence of the $\mathrm{m}$. lumbricalis brevis hallucis (char. 14.0).

Pipidae

A number of synapomorphies have been proposed for this family, including characters from osteology, adult and larval morphology, and musculature (see revisions in Cannatella and Trueb, 1988; Ford and Cannatella, 1993; Trueb et al., 2005; Frost et al., 2006). Not all previously proposed synapomorphies are concordant, and neither are the phylogenetic hypotheses from which they were inferred, but a full discussion of their current phylogenetic distribution is beyond the scope of the present study. Nevertheless, from these sets of synapomorphies, we will discuss the two proposed from hand musculature (Burton, 1998b) and propose an additional one: the absence of the slip with origin on metacarpal III of the medial $\mathrm{m}$. dorsometacarpalis proximalis digiti IV (char. 13.0).

For Pipidae, Burton (1998b) described a dorsal origin of the $\mathrm{mm}$. flexores minimi digitorum III and IV with respect to the mm. intermetacarpales I and II, respectively. Our data do not support this assertion. We compare below the variation in the relationship between the $\mathrm{m}$. flexor minimus digiti IV and $\mathrm{m}$. intermetacarpalis II (our char. 9) and the similar character for the relationship between the $\mathrm{m}$. flexor minimus digiti III and the $\mathrm{m}$. intermetacarpalis I for our sample of pipids. 
Burton (1998b) studied Hymenochirus boettgeri, H. camerunensis, Pipa arrabali, Xenopus laevis, $X$. muelleri, and $X$. tropicalis, among which we also studied $H$. boettgeri, $P$. arrabali, $X$. laevis, and $X$. muelleri. We observed a dorsal origin for the mm. flexores minimi digitorum III and IV with respect to the $\mathrm{mm}$. intermetacarpales I and II in Hymenochirus and Pseudhymenochirus and a ventral origin in Pipa (pl. 9C) and Xenopus. Based on these data, a dorsal origin is a synapomorphy of Hymenochirini and not Pipidae, as proposed by Burton (1998b). A ventral origin is plesiomorphic under the hypothesis of Jetz and Pyron (2018; see appendix 5). Burton (1998b) scored a dorsal origin for the species of Pipa and Xenopus (contra our observations of a ventral origin). Similar problems to those discussed above for Bombinatoridae might explain our disagreement. Finally, it must be noted that under the alternative topologies recovered for the internal relationships of Pipidae (see review by Bewick et al., 2012), a dorsal origin is still not recovered as a synapomorphy of the family.

\section{Acosmanura}

Several characters from larval morphology have been reported as synapomorphies of this clade (Haas, 2003; Frost et al., 2006). However, only two synapomorphies from adult morphology have been identified: the clavicle not overlapping the scapula in adults (see Lynch, 1973: 147), and the palatine being present. This last character is controversial with regard to the taxonomic distribution and the definition of the primary homology hypothesis, as it might be a synapomorphy of Neobatrachia instead (see discussion in Maglia, 2003: 10; Frost et al., 2006: 186).

Here we add two new synapomorphies for Acosmanura from hand musculature: (1) presence of the $\mathrm{m}$. flexor indicis superficialis proprius (char. 1.1; pls. 2A, 6B-D) and (2) presence of an origin of the $\mathrm{m}$. extensor brevis medius digiti IV from the radiale (char. 12.1; pl. 13). The first character has no instance of homoplasy. The second character proposed as a synapomorphy of Acosmanura has some internal reversals (for details see appendix 5).

\section{Anomocoela}

Here we present the first unique, nonhomoplastic synapomorphy for Anomocoela: absence of the $\mathrm{m}$. contrahentis caput longus distalis (char. 10.0; pls. 9D, 10A). This is an especially relevant discovery in light of the extensive research aimed at this clade and its constituent parts.

Several studies have tested the monophyly and internal relationships of Anomocoela. Maglia (1998) constrained the monophyly of Anomocoela but obtained an internal topology with Megophryidae at the base and Pelodytidae as sister of a polytomy containing Pelobates + Scaphiopus + Spea. Haas's (2003) phylogenetic analysis, based mostly on larval characters, rejected the monophyly of Anomocoela, with only Megophryidae and Pelobatidae forming a clade. Most other studies (García-París et al., 2003; Púgener et al., 2003; Roelants and Bossuyt, 2005; San Mauro et al., 2005; Pyron and Wiens, 2011; Pyron, 2014; Chen et al., 2016; Jetz and Pyron, 2018) recovered a monophyletic Anomocoela, with Scaphiopodidae as the sister taxon of Pelodytidae + (Megophryidae + Pelobatidae). Frost et al. (2006) obtained an alternative internal topology for Anomocoela that comprised two major sister groups: Scaphiopodidae + Pelodytidae and Megophryidae + Pelobatidae. The studies of Haas (2003) and Púgener et al. (2003), both composed mostly of characters from larval morphology, are the only quantitative analyses based on phenotypic characters that included exemplars of the four families of Anomocoela. Most other studies were based only on DNA sequences, although Frost et al. (2006) and Chen et al. (2016) also incorporated Haas's (2003) phenotypic dataset and characters from adult osteology, respectively.

Morphological synapomorphies for Anomocoela and its internal clades are scarce, conflicting, and/or contingent on controversial topologies. Ford and Cannatella (1993) provided two synapomorphies for Anomocoela (as Pelo- 
batoidea), albeit in a narrative form: (1) sternum ossified into a bony style and (2) vertical pupil. The sternum forming a bony style needs to be reassessed since at least some Scaphiopodidae (sister taxon of the remaining Anomocoela) have a cartilaginous sternum (Robovská-Havelková, 2010). The vertical pupil is present in several anuran clades, making its optimization ambiguous on most topologies (e.g., Frost et al., 2006; Pyron and Wiens, 2011; Pyron, 2014; Jetz and Pyron, 2018).

Frost et al. (2006) obtained a single phenotypic synapomorphy for Anomocoela, the separated partes corpores of the cartilago labialis superior (Haas, 2003: char. 87.0). Frost et al. (2006) also discussed four characters from Ford and Cannatella (1993) in support of their Mesobatrachia that, under the topology of Frost et al. (2006), are independently homoplastic synapomorphies of Xenoanura and Anomocoela, respectively. However, under alternative topologies (e.g., Pyron and Wiens, 2011; Pyron, 2014; Jetz and Pyron, 2018) the optimization of these characters is ambiguous. On the basis of Lynch's (1973) interpretation of the character distribution, Frost et al. (2006) proposed the dorsally incomplete cricoid ring as an additional synapomorphy of Anomocoela, albeit convergent in Rhinophrynus.

Púgener et al. (2003) obtained one unambiguous synapomorphy for Anomocoela (their Pelobatoidea): the fusion of the suprarostral corpus and alae (also obtained as a synapomorphy for Pipidae), a character state also present in several clades across Anura (e.g., see Haas, 2003). Additionally, different types and degrees of fusion have been reported, including the absence of connecting cartilage cells between the pars alaris and pars corporis (Haas, 1995: 242), different extensions of the distal notch between the partes, and the presence of cartilaginous bridges between the partes, which can appear during development (Haas, 2003: 71). In conclusion, the absence of $\mathrm{m}$. contrahentis caput longus distalis (char. 10.0) is the first nonhomoplastic synapomorphy identified for Anomocoela.
Scaphiopodidae

Frost et al. (2006) recovered several morphological synapomorphies for this family and discussed others from the literature. We found an additional, nonhomoplastic synapomorphy for Scaphiopodidae: the presence of a supplementary origin of the lateral $\mathrm{m}$. lumbricalis brevis digiti IV from metatarsal IV (char. 15.1). Nevertheless, Dunlap (1960: 36) described an origin closely connected with the lateral border of the distal tendon of the $\mathrm{mm}$. flexores breves superficiales (in agreement with our observations) and from a heavy strip of the aponeurosis plantaris at the base of metatarsal V (a tendon from metatarsal IV in our data) in Spea hammondii (the single species of the family of his sample). We studied only a single specimen of Spea hammondii, so we cannot discard the possibility of intraspecific variation. However, we believe that incorrect observation of the origin from metatarsal IV is more likely to explain the discrepancy between our results and those of Dunlap (1960), since the tendon that originates from metatarsal IV is easily confounded with the connective tissue at the base of metatarsal $\mathrm{V}$ and connective tissue of the adjacent aponeurosis plantaris, which is the origin described by Dunlap (1960). Removal of this connective tissue clearly exposes the origin from metatarsal IV.

Pelodytidae + Pelobatidae + Megophryidae

The presence of a slip of the m. extensor brevis medius digiti III originating on the mediodistal end of the radioulna (char. 11.1; pl. 13) optimizes as a synapomorphy of Pelodytidae + Megophryidae + Pelobatidae. The only possible instance of homoplasy is restricted to Craugastor fitzingeri (Craugastoridae); however, the nature of the slip in this species differs (see comments on char. 11). Most analyses (see discussion of Anomocoela, above) have recovered Scaphiopodidae as sister to the remaining anomocoelans (Pelodytidae + Megophryidae + Pelobatidae), which is consistent with the taxonomic distribution of the slip of the $\mathrm{m}$. extensor brevis medius digiti III from the radioulna. 


\section{Megophryidae}

Here we propose a synapomorphy from the palmar musculature (char. 7.1: presence of a supplementary medial slip of the medial $\mathrm{m}$. lumbricalis brevis digiti IV; pl. 7), having examined representatives of each nominal genus. Ford and Cannatella (1993) identified three synapomorphies from adult morphology, although their taxon sampling was restricted to a single species of Leptobrachium and two species of Megophrys (no data matrix presented by Ford and Cannatella, 1993; taxon sampling inferred from unpublished dissertation of Cannatella, 1985, cited by Ford and Cannatella, 1993). Frost et al. (2006) recovered two character states from Haas (2003) as synapomorphies of Megophryidae. However, as was the case with Ford and Cannatella (1993), the data for Megophryidae were restricted to a single species each of Leptobrachium and Megophrys.

Neobatrachia

Our finding that the absence of the $\mathrm{m}$. contrahentis pedis digiti $\mathrm{V}$ (char. 16.0) is a synapomorphy of Neobatrachia is significant, since this character is taxonomically well sampled and nonhomoplastic. Ford and Cannatella (1993) proposed several other synapomorphies for this clade, including (1) the presence of a palatine bone; (2) fusion of the third distal carpal to other carpals; (3) complete separation of the $\mathrm{m}$. sartorius from the $\mathrm{m}$. semitendinosus (also recovered as a synapomorphy by Frost et al., 2006); (4) the presence of an accessory head of the $\mathrm{m}$. adductor longus; and (5) the absence of the parahyoid bone. Some of these suggested synapomorphies deserve comments.

The presence of palatine has been considered a character of central importance for the recognition of Neobatrachia. However, its presence in Anomocoela has not been decisively corroborated or refuted (for summaries see Maglia, 2003, and Frost et al., 2006), so it is not clear whether it is a synapomorphy of Acosmanura (if the anomocoelan and neobatrachian conditions are homologous; Haas, 2003; Frost et al., 2006) or Neobatrachia (if the conditions are not homologous; e.g., Ford and Cannatella, 1993; Trueb, 1993; Púgener et al., 2003).

The fusion of the distal carpal 3 to the other distal carpals has instances of homoplasy that might affect its optimization. Within Neobatrachia, a free distal carpal 3 was reported for Telmatobufo and Calyptocephalella (Calyptocephalellidae), some Arthroleptidae and Myobatrachidae, and Sooglossidae (Fabrezi, 1992; Muzzopappa et al., 2016). Thus, the presence of a distal carpal 3 free in Calyptocephalellidae, Myobatrachidae, and Sooglossidae makes the optimization ambiguous for Neobatrachia on some topologies (e.g., Pyron, 2014; Jetz and Pyron, 2018).

The presence of an accessory head of the $\mathrm{m}$. adductor longus also requires comment. Ford and Cannatella's (1993) description and taxonomic distribution of this character suggest they intended to refer to the $\mathrm{m}$. adductor magnus. To our knowledge, no accessory head has been described for the $\mathrm{m}$. adductor longus, and the taxonomic distribution of the character referring to this muscle does not optimize as a synapomorphy of Neobatrachia (see Dunlap, 1960: 9-11; Cannatella, 1985: char. 121). In contrast, Cannatella (1985: char. 122) described an accessory head of the $\mathrm{m}$. adductor magnus and reported it for his neobatrachian taxa, in agreement with the taxonomic distribution reported by Dunlap (1960: 11). Considering the data of Dunlap (1960) and Cannatella (1985), there is at least one instance of homoplasy regarding the presence of the accessory head of the m. adductor magnus, since it is absent in Hypopachus variolosus (Microhylidae; Dunlap, 1960).

The absence of a parahyoid bone as a synapomorphy of Neobatrachia also presents problems. The only anomocoelan reported to possess a parahyoid bone is Pelodytes caucasicus (Pelodytidae), being absent in the other families (Megophryidae, Pelobatidae, Scaphiopodidae; see Cannatella, 1985: char. 170). This bone is also absent in pipids (Maglia, 1998; Cannatella, 1985). 
With this taxonomic distribution, the optimization of the presence of the parahyoid bone is ambiguous on some topologies (e.g., Pyron, 2014; Jetz and Pyron, 2018), having either been lost in pipids and Acosmanura independently or been lost in Xenoanura + Acosmanura and arisen independently in Rhinophrynus.

\section{Heleophrynidae}

Ford and Cannatella (1993) suggested the absence of larval keratinous jaw sheaths as a synapomorphy of Heleophrynidae (although Heleophryne rosei retains the lower jaw sheath; Channing, 2003). Similarly, Channing (2003) described the development of folds of loose skin during the reproductive aquatic period in Heleophrynidae. Frost et al. (2006) suggested these two characters and 10 larval character states from Haas (2003) as synapomorphies of the family.

Here we add another synapomorphy from the palmar musculature: the presence of the $\mathrm{m}$. lumbricalis longus digiti III (char. 2.1; pl. 6C). This character was previously defined and discussed by Burton (1998b), who reported its presence in Telmatobius brachydactylus and $T$. macrostomus (Telmatobiidae), Calyptocephalella and Telmatobufo (Calyptocephalellidae), Hadromophryne natalensis and Heleophryne purcelli (Heleophrynidae), Odontobatrachus (Odontobatrachidae; as Petropedetes), and Petropedetes (Petropedetidae). Aguilar and Valencia (2009) subsequently reported this muscle in several species of Telmatobius. However, Barrionuevo (2017) reassessed the occurrence of the $\mathrm{m}$. lumbricalis longus digiti III in the species of Telmatobiidae studied by Burton (1998b) and Aguilar and Valencia (2009) and concluded that it is only present in T. macrostomus. Barrionuevo (2017) pointed out that in the other species the $\mathrm{m}$. lumbricalis longus digiti III might have been confused with the slip of the $\mathrm{m}$. lumbricalis brevis digiti III that originates on the tendo superficialis digiti III and/or the flexor plate (our char. 5). Here we confirm the presence of the $\mathrm{m}$. lumbricalis longus digiti III in the two species of Heleophryni- dae studied by Burton (1998b) and in Heleophryne orientalis, and we propose it as an additional synapomorphy for the family.

\section{Sooglossidae}

A number of phenotypic synapomorphies have been proposed for Sooglossidae (e.g., Ford and Cannatella, 1993; Frost et al., 2006; Nussbaum and $\mathrm{Wu}, 2007)$. Those synapomorphies share the problem of the unknown character state in its sister taxon, Nasikabatrachidae, which also affects the synapomorphies we propose below.

Lynch (1973) reported the absence of the columella in Sooglossidae and Pereyra et al. (2016) extended this to Sooglossoidea on the basis of Senevirathne et al.s (2016) report of the absence of the columella in Nasikabatrachus. Following Lynch (1973), Frost et al. (2006) also suggested the occurrence of an ossified omosternum as a synapomorphy of Sooglossoidea (as Sooglossidae). Frost et al. (2006) recovered this clade as sister taxon of Notogaeanura, and thus distantly related to several ranoid families characterized by an ossified omosternum (e.g., Lynch 1973: 146; Scott, 2005: character 28). However, this putative synapomorphy must be reevaluated, since recent hypotheses based on different analytical assumptions recover Sooglossoidea as sister taxon of Ranoides (e.g., Pyron, 2014; Jetz and Pyron, 2018).

On the basis of Nussbaum (1982), Ford and Cannatella (1993) considered the os sesamoides tarsale to be the only known synapomorphy of Sooglossidae. Ford and Cannatella (1993) also suggested a number of synapomorphies for Sooglossidae + Myobatrachidae: (1) ventrally incomplete cricoid ring; (2) semitendinosus tendon inserting dorsal to the $\mathrm{m}$. gracilis; (3) horizontal pupil; (4) winglike alary processes of the hyoid; and (5) divided sphenethmoid. However, in light of the distant phylogenetic position of these two taxa in all recent analyses (e.g., Frost et al., 2006; Pyron and Wiens, 2011; Pyron, 2014; Jetz and Pyron, 2018), these character states are convergent synapomorphies for Sooglossidae (or 
Sooglossoidea) and Myobatrachidae. The pupil was described as horizontal (Biju and Bossuyt, 2003) or round (Radhakrishnan et al., 2007) in Nasikabatrachus sahyadrensis and round in N. bhupathi (see Janani et al., 2017). These discrepancies might be due to descriptions made during pupillary constriction (miosis) or dilation (mydriasis), which in some species can result in different pupil shapes. For example, Barrio (1968: fig. 4) showed a vertical pupil in miosis and round in mydriasis in Lepidobatrachus asper (Ceratophryidae); data considering this fact are required for Nasikabatrachus. Additionally, and similar to the scenario of the ossified omosternum discussed above, a reevaluation of the taxonomic distribution of this character is required considering Ranoides as the sister taxon of Sooglossoidea, as recovered in recent analyses (e.g., Pyron, 2014; Jetz and Pyron, 2018). Additional studies including Nasikabatrachus are required to test if the remaining characters from Ford and Cannatella (1993) optimize as synapomorphies of Sooglossidae or Sooglossoidea.

Nussbaum and Wu (2007) proposed 24 morphological synapomorphies for Sooglossidae in their phylogenetic analysis of this family; however, they did not include Heleophrynidae or Nasikabatrachidae in their analysis. The evaluation of these character states in Heleophrynidae is crucial in light of its phylogenetic position in recent phylogenies, particularly that of Pyron and Wiens (2011), since Sooglossoidea is sister to all Neobatrachia except Heleophrynidae.

Two salient natural history features characterize the species of Sooglossidae: direct development and female parental care, as reported for Sechellophryne gardineri and Sooglossus sechellensis and suspected for S. thomasseti (see Nussbaum and $\mathrm{Wu}, 2007)$. In contrast, Nasikabatrachidae (Raj et al., 2012; Zachariah et al., 2012; Janani et al., 2017) and Heleophrynidae (Channing et al., 2012) have free-living larvae and lack parental care. In the context of the alternative phylogenetic positions recovered for Sooglossidae + Nasikabatrachidae (e.g., Frost et al., 2006; Pyron and Wiens, 2011; Pyron, 2014;
Jetz and Pyron, 2018), direct development and female parental care are two additional synapomorphies for the family.

Unfortunately, the four characters from the current study that might optimize as synapomorphies of Sooglossidae (chars. 8.1, 17.0, 19.1, 20.1) are also compromised by the lack of evidence for Nasikabatrachus and Sechellophryne. The absence of the m. extensor brevis superficialis digiti III of the pes (char. 17.0) and presence of the m. flexor minimus indicis (char. 8.1) have the fewest instances of homoplasy (appendices 4 and 5). The other two character states from the dorsal foot musculature $(19.1,20.1)$ show an unusual pattern of homoplasy and are further discussed below in the section of Laurentobatrachia.

\section{Laurentobatrachia}

The larval $\mathrm{m}$. levator mandibulae externus in two portions (Haas, 2003: char. 54.1), the absence of commissura proximalis III (Haas, 2003: char. 111.0), and the absence of intercalary elements (Haas, 2003: char. 151.0; however, it is ambiguous following Manzano et al., 2007) are probably synapomorphies of this group (Frost et al., 2006: 232). Claw-shaped terminal phalanges (Liem, 1970), thyrohyals on cartilaginous stalks (Drewes, 1984: char. 10.1), and external metatarsal tubercle absent or poorly developed (Laurent, 1986) are also synapomorphic for this clade (see discussion in Frost et al., 2006: 232).

Here, we add two new synapomorphies involving two previously unreported muscles: the presence of $\mathrm{mm}$. abductores breves dorsales digitorum II and III (chars. 19.1 and 20.1, respectively; pl. 14). What is particularly striking is that both characters have almost identical taxonomic distributions (see appendix 5).

This pattern could imply that both character states are dependent and should be coded as a single transformation series. However, the presence of the $\mathrm{m}$. abductor brevis dorsalis digiti II (char. 19.1) and apparent absence of the $\mathrm{m}$. abductor brevis dorsalis digiti III (char. 20.0) in Leptobrachella bourreti (Megophryidae) establishes the transformational independence of the 
two characters. Nevertheless, the absence in $L$. bourreti should be corroborated in additional specimens, since the nature of the available material (a combination of its small size, unpigmented muscles, and scarce elasticity of the muscular fibers) precluded a fully confident scoring in the single foot of the exemplar studied.

\section{Calyptocephalellidae}

Frost et al. (2006), on the basis of data from Burton (1998b), proposed the following synapomorphies for their Batrachophrynidae (composed of Batrachophrynus, Calyptocephalella, and Telmatobufo): (1) presence of $\mathrm{m}$. lumbricalis longus digiti III (our char. 2; pl. 6C) and (2) two origins of the $\mathrm{m}$. lumbricalis brevis digiti III, from the flexor plate and from distal carpals (our chars. 5 and 6). Subsequently, Batrachophrynus was transferred to the distantly related Telmatobius (Telmatobiidae), and Calyptocephalellidae was resurrected for Calyptocephalella and Telmatobufo (Aguilar and Valencia, 2009). This fact by itself does not affect the status of these character states as possible synapomorphies for the family. However, some clarifications and reinterpretations are required.

Burton (1998b: char. 3) scored the presence of both slips of the $\mathrm{m}$. lumbricalis brevis digiti III (the slip from the distal carpals plus the slip from the flexor plate/tendo superficialis digiti III) within the same transformation series: (0) originating from the flexor plate/tendo superficialis digiti III only; (1) two origins: from the flexor plate/tendo superficialis digiti III, and from the distal carpals; (2) originating from the distal carpals only. We consider each slip with each origin to be independent and, consequently, score their occurrence as separate characters. By employing this coding scheme, it is evidenced that Calyptocephalellidae is not supported by any of the characters referring to the origin of the $\mathrm{m}$. lumbricalis brevis digiti III, since (1) the presence of the slip of the $\mathrm{m}$. lumbricalis brevis digiti III from the flexor plate/tendo superficialis digiti III is plesiomorphically present (char. 5.1; see appendix 5 for ACSR), and (2) the presence of the slip of the $m$. lumbricalis brevis digiti III from the distal carpals is also plesiomorphically present (char. 6.1; see appendix 5).

Myobatrachoidea

Frost et al. (2006) did not identify any morphological synapomorphy for this superfamily. However, and in line with the above discussion for Calyptocephalellidae, under the coding scheme that treats the slip of the $\mathrm{m}$. lumbricalis brevis digiti III from the flexor plate/tendo superficialis digiti III (char. 5) and the slip from the distal carpals (char. 6) as independent characters (contra Burton, 1998b), the absence of the $\mathrm{m}$. lumbricalis brevis digiti III from the distal carpals is a synapomorphy of Myobatrachoidea (char. 6.0). A reversion occurs in the limnodynastids Lechriodus + Platyplectrum (m. lumbricalis brevis digiti III from distal carpals present).

\section{Nobleobatrachia}

This taxon, erected by Frost et al. (2006) on the basis of molecular synapomorphies only, accounts for more than the half of all anuran species. Frost et al. (2006) discussed two ambiguously optimized morphological character states: the presence of claw-shaped terminal phalanges and intercalary elements. Here we identify the first unambiguously optimized morphological synapomorphy for this major clade, the $\mathrm{m}$. flexor minimus digiti IV dorsal to the $\mathrm{m}$. intermetacarpalis II (char. 9.1; pls. 2D, 10B; for instances of homoplasy see appendix 5 and Burton, 1998b).

\section{Homology And Terminology: A REAPPRAISAL}

As mentioned above in Materials and Methods, most studies of anuran musculature are based on the classical terminology of Gaupp (1896). Recently, Abdala and Diogo (2010), Diogo and Abdala (2010), Diogo and Molnar (2014), Diogo and Ziermann (2014), and Diogo et al. (2018) discussed and introduced several modifications intended to unify terminology 
across tetrapods. Below, we review these terminological suggestions for Anura and other relevant aspects linking homology and terminology (see also Terminology in Materials and Methods, above), discuss some persistent controversies, and introduce several modifications.

Our discussion is organized in the following order: (1) palmar muscles, (2) dorsal hand muscles, (3) plantar muscles, (4) dorsal foot muscles, and (5) related muscles of the hand and foot (for muscle groups with equivalent problems in both regions).

M. flexor indicis superficialis proprius, M. caput profundum digiti III, and M. flexor accessorius: Novelties from Splitting?

The $\mathrm{m}$. flexor indicis superficialis proprius and $\mathrm{m}$. caput profundum digiti III are two muscles with unique names that are not arranged into groups (e.g., mm. lumbricales breves digitorum II-V). However, the taxonomic distribution of their absence/presence, degree of development, and coexistence or not with the portion of the $\mathrm{m}$. flexor accessorius that originates on the distal carpals suggests that these two muscles evolved as differentiations of distal slips of the $\mathrm{m}$. flexor accessorius.

The $\mathrm{m}$. flexor indicis superficialis proprius originates on the distal carpals and inserts via a long flexor tendon on the palmar surface of the distal phalanx (see description of Triprion petasatus, char. 1 , and pls. 2A, 6B-D). This muscle is absent in nonacosmanuran clades, where the tendo superficialis indicis is found in this topological region, probably fulfilling the function of a flexor tendon that attaches to the distal phalanx. The $\mathrm{m}$. caput profundum digiti III has the same characteristics as the $\mathrm{m}$. flexor indicis superficialis proprius (see pls. 2A, $6 \mathrm{~B}-\mathrm{D})$, and it is also absent in early diverging clades (Burton, 1998b; this study).

The $\mathrm{m}$. flexor accessorius arises typically on the laterodistal end of the radioulna. However, in early diverging clades, more distal origins on the ulnare and distal carpals also occur (e.g., Ascaphidae + Leiopelmatidae, Costata, Xenoanura, some Anomocoela, Heleophrynidae, some Australobatrachia; Ribbing, 1911; this study; see also the legend of pl. 9C). The inser- tion is also relevant for the hypothesis discussed here, since it occurs on the dorsal surface of the flexor plate, reaching the proximal and dorsal end of the tendines superficiales of digits II-IV in some species. Thus, the m. flexor accessorius has the same connections as the $\mathrm{m}$. caput profundum digiti III in some species: an origin on the same region of the distal carpals (at the level of metacarpal III) and an insertion on the dorsal surface of the tendo superficialis of digit III.

Burton (1998b) suggested that the $\mathrm{m}$. caput profundum digiti III derives from the $m$. flexor accessorius, without further comments. We found substantial evidence for Burton's (1998b) assertion. There is a correlation between the absence of the portion of the $\mathrm{m}$. flexor accessorius with origin on distal carpals and the presence of a well-developed and differentiated caput profundum digiti III. There are also intermediate morphologies, where the $\mathrm{m}$. caput profundum digiti III absent (e.g., Rhinophrynidae; pl. 6A) or reduced (e.g., Heleophryne purcelli; pl. 6C) in the presence of the portion from the carpals of the $\mathrm{m}$. flexor accessorius, to the extent that there are species in which it is unclear if the m. caput profundum is present and reduced or the portion from the distal carpals of the m. flexor accessorius is present instead (e.g., some Australobatrachia and Scaphiopodidae).

The $\mathrm{m}$. flexor indicis superficialis proprius has a similar morphology, connections, and functions to the $\mathrm{m}$. caput profundum digiti III. We extend the above-discussed hypotheses to this muscle, suggesting that it also arose as a slip differentiated from the $\mathrm{m}$. flexor accessorius.

The $\mathrm{m}$. flexor indicis superficialis proprius evolved as a discrete muscle in Acosmanura. It coexists with the portion of the $\mathrm{m}$. flexor accessorius from the distal carpals in some acosmanurans (e.g., some Anomocoela, Heleophrynidae, some Australobatrachia). Similarly to the m. caput profundum digiti III, the $\mathrm{m}$. flexor indicis superficialis proprius is usually absent or reduced in those clades in which the portion of the $\mathrm{m}$. flexor accessorius from the distal carpals is present (e.g., pl. 6C). 
At this point, other lines of evidence would prove useful for testing this hypothesis. First, although innervation in Pelophylax (Ranidae) reported by Gaupp (1899: 180) supports this hypothesis (the same nerve innervates the three muscles), data on its variation across Anura is required. Second, a formal quantitative test (e.g., intermediate morphologies as discussed by Ramírez, 2007: 591) is required, comparing the taxonomic distribution and detailed morphology of these muscles. And third, developmental data are required to determine if these muscles derive from the same anlage.

Is the Anuran M. flexor accessorius (Originating on Carpals) Homologous to the Caudate M. flexor accessorius medialis?

A problem that cannot be overlooked is the putative homology of the anuran $\mathrm{m}$. flexor accessorius and the caudate $\mathrm{mm}$. flexores accessorius medialis and lateralis. The anuran $\mathrm{m}$. flexor accessorius originates on the laterodistal end of the radioulna, as well as the ulnare and the distal carpals in early diverging clades; it inserts on the flexor plate and, in some species, also proximally on the more preaxial tendines superficiales (see further details in previous section).

On the basis of its position and attachments, Ribbing (1907: 597) considered that the single $\mathrm{m}$. flexor accessorius of Anura probably corresponds to the $\mathrm{m}$. flexor accessorius lateralis of Caudata. Later, in his study of Pipa cf. pipa, Ribbing (1911: 11-12, 24) hypothesized that the portions of the $\mathrm{m}$. flexor accessorius that originate on the carpals and the radioulna correspond to the $\mathrm{m}$. flexor accessorius medialis and $\mathrm{m}$. flexor accessorius lateralis of Caudata, respectively. Abdala and Diogo (2010: 25) and Diogo and Abdala (2010: 402), quoting Ribbing (1907), stated that the $\mathrm{m}$. flexor accessorius of anurans, such as the species of Bufonidae they studied, "topologically corresponds to the flexor accessorius medialis plus flexor accessorius lateralis of urodeles such as Ambystoma." However, as noted above, Ribbing (1907) considered that the anuran $\mathrm{m}$. flexor accessorius probably corresponds to the caudate m. flexor accessorius lateralis, not both muscles. Diogo et al. (2018) briefly discussed the problem, pointing out the contradictory correspondences between the $\mathrm{m}$. flexor accessorius medialis, $\mathrm{m}$. flexor accessorius lateralis, and m. palmaris profundus I of Caudata with the $\mathrm{m}$. flexor accessorius of Anura.

The medial origin of the $\mathrm{m}$. flexor accessorius medialis with respect to the $\mathrm{m}$. contrahentium caput longum in Caudata (see review by Diogo et al., 2018: figs. 16.3F, 17.4C) might suggest its nonhomology with the distal portion of the $\mathrm{m}$. flexor accessorius that originates on the carpals, as occurs in early diverging clades of anurans. In these anuran clades, the portion of the $\mathrm{m}$. flexor accessorius that originates on the carpals is located lateral and distal (and not medial, as in caudates) to the distal portion of the $\mathrm{m}$. contrahentium caput longum (considered here to be the $\mathrm{m}$. contrahentis caput longus distalis).

Plate 9A-C exemplifies the topological relationship between the $\mathrm{m}$. contrahentis caput longus distalis (i.e., the distal portion of the caudate $\mathrm{m}$. contrahentium caput longum) and the $\mathrm{m}$. flexor accessorius. Although the m. flexor accessorius was removed in the specimens of this plate, its origin occupies the ventral and distal portion of the radioulna, the exposed ventral portion of the ulnare, and the distal carpal(s) at the base of metacarpals IV-V (see legend of pl. 9 for details and variation in each species). As exposed in this plate, the origin of the $\mathrm{m}$. flexor accessorius from the distal carpals is distal to the $\mathrm{m}$. contrahentis caput longus distalis, and not medial as expected for the $\mathrm{m}$. flexor accessorius medialis of caudates. This topological position supports the hypothesis that the muscle in Anura corresponds to the $\mathrm{m}$. flexor accessorius lateralis of Caudata, in agreement with Ribbing (1907). However, detailed comparative studies of the variation of this muscle in early diverging clades of Anura and Caudata are required to test the hypothesis of Ribbing (1911). These studies would require evaluation of, minimally, the relative position of the neighboring muscles, the dif- 
ferences and homology between the $\mathrm{m}$. contrahentium caput longum in anuran and caudates, and innervation.

Identities of the Hand $\mathrm{Mm}$. flexores breves superficiales and Mm. Lumbricales in Anura: A Provisional Solution

Some recent contributions are unclear as to the total or partial correspondence of the anuran $\mathrm{mm}$. lumbricales breves and longi of classical terminology to the mm. flexores breves superficiales of other tetrapods. Additionally, there is confusion with respect to the correspondence between the anuran mm. lumbricales breves and longi. This section discusses and establishes a tentative name for the anuran $\mathrm{mm}$. lumbricales breves and longi of classical terminology, evaluating the names and homology of potentially equivalent muscles in other tetrapods, especially Caudata.

Most conflict revolves around the assertions of Manzano et al. (2008), Abdala and Diogo (2010), Diogo and Abdala (2010), Diogo and Ziermann (2014), and Diogo et al. (2018). We begin by describing each muscle involved in the problem and clarifying their names and identities, as inferred from the abovementioned studies. We then delimit the alternative hypotheses and discuss the evidence and operational considerations that lead us to favor one of them.

DESCRIPTION OF THE MUSCLES: The description is arranged following the layers as defined in Results. Special comments are made for the conditions in Pelophylax cf. esculenta, because this is the model species of Ecker (1864) and Gaupp (1896), frequently cited as providing support for the assertions of the different authors discussed here. Additionally, we discuss the variation in these muscles across the phylogeny and comment on the plesiomorphic condition in Anura when relevant.

M. flexor indicis superficialis proprius (layer 1; pls. 2A, 6B-D): This muscle was described for the model species Triprion petasatus and in Character 1, and the ACSR is given in appendix 5. It is present in Pelophylax cf. esculenta and plesiomorphically absent in Anura (this study).
M. caput profundum digiti III (layer 1; pls. 2A, 6B-D): This muscle is very similar to the preceding one. It originates on the distal carpals and inserts on the dorsal surface of the tendo superficialis digiti III, forming a common tendon of insertion that attaches to the distal phalanx. It is present in Pelophylax cf. esculenta and plesiomorphically absent in Anura (Burton, 1998b; this study).

$\mathrm{Mm}$. lumbricales longi digitorum III-V (layer 1; pls. 2A, 6A-D): These muscles originate on the flexor plate and/or tendines superficiales of their corresponding digit. They insert on the basal phalanx (muscle of digit III; pl. 6C) or proximal interphalangeal joint (muscles of digits IV-V; pl. $6 \mathrm{~A}-\mathrm{D}$ ). The main variation involves the differentiation into a medial and lateral slip and insertion via one or two tendons (e.g., Burton, 1996, 1998a, 1998b; this study). The muscle of digit III was described in Character 2 and the ACSR is given in appendix 5; it is plesiomorphically absent in Anura. The muscles of digits IV and V are present in almost all anurans (including Pelophylax cf. esculenta) and are plesiomorphically present in Anura (Burton, 1998b; this study).

Mm. lumbricales breves digitorum II-V (layer 2; pls. 2B, 6A-D, 7): These muscles are almost invariably present in Anura; they originate on the distal carpals, flexor plate, and/or tendines superficiales of their respective digit, and insert on the metacarpophalangeal joint and/or the proximal end of basal phalanx (in arboreal clades a single or additional insertion on the distal end of their respective metacarpal may occur in digits IV and V; this study). In Pelophylax cf. esculenta, at least partial portions or slips of the muscles of digits II-V originate on the flexor plate (Gaupp, 1896).

The muscles of digits II-V originate plesiomorphically from the dorsal surface of the flexor plate in Anura (this study; see chars. 3-6 for the muscles of digit II and III). The identity of the lateral $\mathrm{m}$. lumbricalis brevis digiti $\mathrm{V}$ is conflictive in several species (see appendix 3).

The flexor plate is absent or reduced in arboreal clades (e.g., Allophrynidae + Centrolenidae, 
Hemiphractidae, Hylidae, Hyperolius, Leptopelinae, and Rhacophoridae; Liem, 1970; Burton, 1996; Faivovich, 2002: fig. 13; Manzano et al., 2008; Araujo-Vieira et al., 2019; this study) and the $\mathrm{mm}$. lumbricales breves of digit IV originate on tendo superficiales digiti IV instead. In those clades, the medial and lateral muscles of digit IV are usually reduced in volume, and one of them may be absent (usually the lateral; e.g., see pl. 2B).

Discussion of THE PROBLEM: Two partially independent problems regarding the identities of this group of muscles are addressed in this section. First, we discuss the identities of the $\mathrm{mm}$. lumbricales breves and longi of digit IV in arboreal clades in light of Manzano et al's (2008) reports. Second, we address whether the $\mathrm{mm}$. flexores breves superficiales and/or mm. lumbricales of other tetrapods are present in Anura, discussing the positions of Abdala and Diogo (2010), Diogo and Abdala (2010), Diogo and Ziermann (2014), and Diogo et al. (2018) in this respect.

The identities of the $\mathrm{mm}$. lumbricales breves and longi of digit IV in arboreal clades: Manzano et al. (2008) described the m. lumbricalis longus digiti IV as comprising two internal branches (inserting on penultimate phalanx IV) and two external (inserting on distal end of metacarpal IV). However, we consider that their internal and external branches correspond to the $\mathrm{m}$. lumbricalis longus and mm. lumbricales breves, respectively. The problems with the correspondence between the $\mathrm{mm}$. lumbricales longi and the mm. lumbricales breves arise when the lumbricales breves originate on the tendo superficiales and not the flexor plate, as usual. However, the origin of the mm. lumbricales breves on the tendo superficialis of digit IV in arboreal clades (like the species studied by Manzano et al., 2008 ) is common (see description above), and is discussed below.

According to our interpretation, in the nonarboreal clades that are closely related to the arboreal Allophrynidae + Centrolenidae, Hemiphractidae, Hylidae, Hyperolius, Leptopelinae, and Rhacophoridae, respectively, the medial and lateral $\mathrm{m}$. lumbricales brevis of digit $\mathrm{IV}$ originate on the flexor plate (this study). In these arboreal clades, the mm. lumbricales breves of digit IV maintains the origin in the same topological region (i.e., the tendo superficialis, due to the reduction of the flexor plate), as well as similar morphologies, connectivities, and presumed functions, as in the non-arboreal clades to which each of them are related.

An alternative hypothesis, consistent with the interpretation of Manzano et al. (2008), is that the $\mathrm{mm}$. lumbricales breves of digit IV of the hylids studied by Manzano et al. (2008) are lost and the $\mathrm{m}$. lumbricalis longus is differentiated into additional medial and lateral slips inserting on the distal end of metacarpal IV. However, this is a less parsimonious explanation, requiring the simultaneous (i.e., on the same phylogenetic branch) loss of the mm. lumbricales breves of digit IV and the origin of novel supplementary medial and lateral slips of the $\mathrm{m}$. lumbricalis longus in approximately the same region, with similar origins, insertions, and presumed functions as the $\mathrm{m}$. lumbricalis brevis of other species/clades. Furthermore, this would need to be postulated not only for Hylidae (as the species studied by Manzano et al., 2008), but also for all the arboreal clades mentioned above, for which these novelties would have had to evolve independently.

The identities of the anuran $\mathrm{mm}$. lumbricales breves and longi: The purpose of this section is to analyze in detail the several, sometimes contradictory, comments on these muscle groups by Abdala and Diogo (2010), Diogo and Abdala (2010), Diogo and Ziermann (2014), and Diogo et al. (2018). Abdala and Diogo (2010) make confusing statements about the identity of the $\mathrm{mm}$. flexores breves superficiales and mm. lumbricales. First, they state that "the flexores breves superficiales are consistently present in limbed amphibians and reptiles, forming a muscular complex that often originates from the flexor retinaculum and/or carpal bones and inserts onto the distal phalanges" (Abdala and Diogo, 2010: 31). However, neither the $\mathrm{mm}$. lumbricales longi nor the $\mathrm{mm}$. lumbricales breves attach to the distal phalanges. The only 
palmar muscles attaching to the distal phalanges are the $\mathrm{m}$. flexor indicis superficialis proprius and the $\mathrm{m}$. caput profundum digiti III, both hypothesized here to be derived from the $\mathrm{m}$. flexor accessorius (see above).

Furthermore, Abdala and Diogo (2010: 31) state that "in amphibians the flexores breves superficiales have a particular conformation because they are often markedly reduced and mainly associated to the structure that is often designated as 'palmar aponeurosis' in the literature (e.g. Ecker, 1889; Walthall and Ashley-Ross, 2006)." The reference to Ecker (1889) and its origin on the "palmar aponeurosis" (= flexor plate) suggest they are referring to the $\mathrm{mm}$. lumbricales breves, although, as mentioned above, those muscles do not insert on the distal phalanges.

Regarding the mm. lumbricales, Abdala and Diogo (2010: 32) state that "the lumbricales are small muscles that often run from the tendons of the flexors of the forearm to the distal phalanges of the digits, and which are usually present in anurans, turtles, lepidosaurs and crocodylians, but absent in most urodeles and seemingly also in birds." However, the mm. lumbricales (breves and longi) do not attach to the distal phalanges. Subsequently, on the same page, they state more specifically that "anurans often have lumbricales and, in at least some cases, including the Phyllomedusa bicolor specimens dissected by us, these muscles are differentiated into 'lumbricales breves' and 'lumbricales longi' (e.g., Gaupp, 1896; Manzano, 1996; this work)." This statement is incongruent with the presence of the $\mathrm{mm}$. flexores breves superficiales, because if the $\mathrm{mm}$. lumbricales breves and longi of Gaupp (1896) are considered both to be $\mathrm{mm}$. lumbricales, then there is no other muscle group attributable to the $\mathrm{mm}$. flexores breves superficiales.

Diogo and Abdala (2010: 379) explicitly accept the presence of the $\mathrm{mm}$. flexores breves superficiales in Anura by listing them in their table 10.1, but they are not described, and it is not indicated to which muscles of other authors they correspond. Next, regarding the $\mathrm{mm}$. lumbricales, Diogo and Abdala (2010: 380) state that "our dissections of anurans such as Phyllomedusa bicolor show that these anurans have both 'lumbricales breves' and 'lumbricales longi': see also, e.g., Gaupp 1896 and Manzano 1996." As discussed above for Abdala and Diogo (2010), if the $\mathrm{mm}$. lumbricales breves and longi of Gaupp (1896) are both considered to be mm. lumbricales, then there is no other muscle group attributable to the $\mathrm{mm}$. flexores breves superficiales. Furthermore, their mm. flexores breves superficiales were not described, precluding the identification of their concept of this muscle group.

In contrast, Diogo and Ziermann (2014: table 2) are clear with respect to their concept of the $\mathrm{mm}$. flexores breves superficiales. They consider the $\mathrm{mm}$. lumbricales breves and longi of Duellman and Trueb (1986), who followed Gaupp (1896), to correspond to their mm. flexores breves superficiales and $\mathrm{mm}$. lumbricales, respectively. However, the $\mathrm{mm}$. lumbricales longi of Gaupp (1896) are superficial to the mm. lumbricales breves of Gaupp (1896), which contradicts the assertions of Diogo and Abdala (2010: 410) and Diogo et al. (2018: 477) that the mm. flexores breves superficiales are superficial to the mm. lumbricales.

Diogo et al. (2018: 477) also comment on the $\mathrm{mm}$. flexores breves superficiales in Anura, duplicating a paragraph of Abdala and Diogo (2010; see above). Diogo et al. (2018: 452) stated that the $\mathrm{mm}$. flexores breves superficiales in Anura are:

part of flexores digitorum breves superficiales sensu McMurrich (1903a,b); flexores breves sublimes sensu Holmes (1977); flexores digiti brevis superficialis sensu Abdala and Moro (2006) (they are often subdivided into bundles as shown by Ecker 1864; four muscles attaching to digits $2-5$, respectively, in the Rana specimens observed by us and as described by, e.g., Ecker 1864).

Their concept of the $\mathrm{mm}$. flexores breves superficiales in anurans is unclear, since McMurrich $(1903 a, 1903 b)$ did not deal with anuran musculature. Similarly, Holmes (1977) and 
Abdala and Moro (2006) did not study anuran muscles or make any explicit statements on their identities. While citing Ecker (1864), Diogo et al. (2018) did not mention to which specific muscles of this author they were referring; at most, two muscle groups of Ecker (1864) might fit their characterization: (1) the $\mathrm{m}$. flexor digiti longus $\mathrm{s}$. sublimis of digits II-V and (2) the m. flexor digiti brevis profundi of digits II-IV and (probably) the flexor digiti $\mathrm{V}$ brevis. The first group corresponds to the $\mathrm{m}$. flexor indicis superficialis proprius, $\mathrm{m}$. caput profundum digiti III, and $\mathrm{mm}$. lumbricales longi digitorum IV-V, and the second to the mm. lumbricales breves (see appendix 3). Since Diogo et al. (2018; see below) considered that the first group corresponds to their $\mathrm{mm}$. lumbricales, we infer that their concept of the mm. flexores breves superficiales might correspond to the mm. lumbricales breves.

Concerning the mm. lumbricales, Diogo et al. (2018: 453) stated that they

mainly correspond to flexor digiti longus sublimi 2, 3, 4, and 5 sensu Ecker (1864) (our dissections of anurans such as Phyllomedusa bicolor show that these anurans have both 'lumbricales breves' and 'lumbricales longi': see also, e.g., Gaupp [1896] and Manzano [1996]; however, it is possible that part of the 'lumbricales' correspond to bundles of the flexores breves superficiales sensu the present work; four muscles attaching to digits 2-5 in the Rana specimens we dissected).

There are two problems with this statement. First, the mm. flexor digiti longus sublimi of digits II-V of Ecker are the most superficial muscles, contradicting Diogo and Abdala (2010: 410) and their own assertion on p. 477, where it is stated that the $\mathrm{mm}$. flexores breves superficiales are superficial with respect to the $\mathrm{mm}$. lumbricales. Second, the mm. flexor digiti longus sublimi of digits II-V of Ecker are a composite of two (if the muscles of digits II and III are derived from the $\mathrm{m}$. flexor accessorius, as hypothesized here) or three unrelated muscle groups: the m. flexor indicis superficialis proprius, m. caput profun- dum digiti III, and $\mathrm{mm}$. lumbricales longi digitorum IV-V. Furthermore, as discussed above for Abdala and Diogo (2010) and Diogo and Abdala (2010), if the mm. lumbricales breves and longi of Gaupp (1896) correspond to their mm. lumbricales, there is no other superficial muscle group attaching to the four digits to be identified as the $\mathrm{mm}$. flexores breves superficiales.

Similar problems are implicit in Diogo et al. (2018: table 19.19). In this table they establish topological correspondences between the foot and hand muscles of a representative of Anura (Xenopus laevis) and Caudata (Ambystoma mexicanum). They explicitly consider the $\mathrm{mm}$. lumbricales of the hand to be composed of $\mathrm{mm}$. lumbricales breves and longi, while the identity of the flexores breves superficiales is not clearly stated, besides the indication of its topological correspondence with the homonym muscle of the foot. However, the mm. flexores breves superficiales of the foot are, despite its plural designation, usually a single, large muscle with a single tendon of insertion (even in Xenopus laevis) that splits into the tendines superficiales of digits III-V that attach to the distal phalanges of these digits. The only topologically similar muscle of the hand, if any, is the $\mathrm{m}$. flexor digitorum communis, which usually inserts via tendons that split into the tendines superficiales of digits III-V, that, as in the foot, attach to the distal phalanges either directly (as in Triprion petasatus and most arboreal clades) or indirectly through the formation of a flexor plate; that plate is a strong tendon of insertion of this muscle as well as the $\mathrm{m}$. flexor accessorius, and serves as origin for the tendines superficiales.

In light of our last statement, a new possibility emerges: the mm. flexores breves superficiales of Diogo and associates are the $\mathrm{m}$. flexor digitorum communis. However, this is rejected in all the statements referring to this muscle in all their studies, since their $\mathrm{m}$. flexor digitorum communis is clearly identified as such and corresponds to our homonym muscle. Again, it is not clear which muscles of the hand can be attributed to 
the $\mathrm{mm}$. flexores breves superficiales sensu Diogo et al. (2018) in Anura.

Synthesizing, it is not clear whether the $\mathrm{mm}$. flexores breves superficiales and/or the $\mathrm{mm}$. lumbricales are present in Anura, and it is mandatory to define the names for the two muscle groups in conflict: the $\mathrm{mm}$. lumbricales breves and $\mathrm{mm}$. lumbricales longi (sensu both Gaupp, 1896, and this study). Their homologies and correspondences within Anura are clear and beyond discussion. The problem can be circumscribed by the following four competing hypotheses: (1) the mm. lumbricales breves and longi are part of the $\mathrm{mm}$. lumbricales of other tetrapods, implying the absence of the $\mathrm{mm}$. flexores breves superficiales in Anura; (2) the mm. lumbricales breves and longi are part of the $\mathrm{mm}$. flexores breves superficiales and $\mathrm{mm}$. lumbricales of other tetrapods, respectively (as proposed by Diogo and Ziermann, 2014); (3) the mm. lumbricales breves and longi are part of the $\mathrm{mm}$. flexores breves superficiales of other tetrapods, implying the absence of the $\mathrm{mm}$. lumbricales in Anura; (4) the mm. lumbricales. breves and longi are part of the $\mathrm{mm}$. lumbricales and $\mathrm{mm}$. flexores breves superficiales, respectively. Below we discuss the pros and cons of adopting each of these possible solutions.

Possible solutions: All four hypotheses have important consequences regarding homology. Hypothesis (1) implies the absence of $\mathrm{mm}$. flexores breves superficiales in Anura and presence of both the mm. lumbricales breves and longi, consistent with classical terminology (e.g., Gaupp, 1896). However, the $\mathrm{mm}$. flexores breves superficiales of Caudata (e.g., see Walthall and Ashley-Ross, 2006) are similar in position, morphology, and attachments to the anuran $\mathrm{mm}$. lumbricales breves (particularly to the early diverging clades), providing evidence for their homology. This putative homology was previously suggested by Burton (1998b: 4).

Hypothesis (2) is rejected because it is in conflict with the relative layer positions of the $\mathrm{mm}$. flexores breves superficiales and $\mathrm{mm}$. lumbricales (see Diogo and Abdala, 2010: 410; Diogo et al., 2018: 477).
Hypothesis (3) implies the absence of $\mathrm{mm}$. lumbricales in Anura, with the mm. Jumbricales breves and longus corresponding to different portions of the $\mathrm{mm}$. flexores breves superficiales. In this case, the mm. flexores breves superficiales of Caudata would correspond to the anuran mm. lumbricales breves and the $\mathrm{mm}$. lumbricales longi would be absent or undifferentiated in Caudata (but see Burton, 1998b: 4 , for a caudate muscle that might be interpreted as homologous to the anuran $\mathrm{m}$. lumbricalis longus of digit IV).

Hypothesis (4) is heterodox, since it was never suggested directly or indirectly in the literature. However, assuming terminology is intended to represent homology, it implies that the $\mathrm{mm}$. flexores breves superficiales of Caudata correspond to the $\mathrm{mm}$. lumbricales longi and not the $\mathrm{mm}$. lumbricales breves of Anura, which is unlikely, as discussed above.

In summary, none of the hypotheses enjoys strong empirical support and all are undercut by either a lack of evidence or contradictory evidence, and we believe it to be both unjustified and counterproductive to rename these muscles without evidence to put the question to rest decisively. Consequently, although we readily acknowledge the shortcomings of Hypothesis 1 , we prefer to retain it as our working hypothesis and employ the corresponding classical terminology (i.e., presence of $\mathrm{mm}$. lumbricales breves and longi in Anura, absence of $\mathrm{mm}$. flexores breves superficiales) until a general revision of these problematic identities is conducted across tetrapods, and especially in the extremely poorly studied cryptobranchoid caudates. Among the several problems to be addressed, the report of putative $\mathrm{mm}$. lumbricales in the third and fourth digit in the caudate Ambystoma by McMurrich (1903a: fig. 10) is especially relevant. Finally, it must be noted that if the putative correspondence suggested here between the $\mathrm{mm}$. lumbricales breves of Anura and the $\mathrm{mm}$. flexores breves superficiales of Caudata is accepted, their homology should be transferred to a separate homology statement (i.e., the mm. lumbricales 
breves of Anura are homologous to the $\mathrm{mm}$. flexores breves superficiales of Caudata).

Hand Mm. contrahentes digitorum: An Overlooked Muscle Group in Anura

This muscle group has not been identified as such in the classical terminology (see appendix 3). It belongs to the third layer (pls. 2C, $8,10 \mathrm{~A}$ ) and is characterized by an origin on the distal carpals and insertion on the basal phalanx (but see discussion below for the muscle of digit V). Historically, some of these muscles were grouped among the $\mathrm{mm}$. flexores digitorum minimi (under the synonym of "flexores teretes," see appendix 3). We believe that a combination of the following points explains the lack of recognition of these muscles as a group.

First, the models for the classical studies are species of Neobatrachia, in which these muscles do not form the complete muscle group found in early diverging clades. The $\mathrm{mm}$. contrahentes are restricted to digits II and $\mathrm{V}$ in most neobatrachians (see pls. 2C and $8 \mathrm{C}$ ), while some species of Ranoides also have an $\mathrm{m}$. contrahentis in digit IV (this study). This lack of a complete muscle sheet might have led the $\mathrm{mm}$. contrahentes to be included in other muscle groups.

Second, the absence of an $\mathrm{m}$. flexor minimus in digit II in almost all Neobatrachia (see further discussion below) might have led the $\mathrm{m}$. contrahentis of digit II to be treated as part of the $\mathrm{mm}$. flexores digitorum minimi. However, the origin on the distal carpals is characteristic of the $\mathrm{mm}$. contrahentes and not the $\mathrm{mm}$. flexores digitorum minimi (defined by its origin on the metacarpals).

Third, the $\mathrm{m}$. contrahentis of digit IV (present in some Ranoides, including the model species of Pelophylax of classical terminology) was also considered part of the $\mathrm{mm}$. flexores digitorum minimi group, possibly because it converges into a common insertion with the true $\mathrm{m}$. flexor minimus of digit IV (appendix 3). Accordingly, Burton (1998b) identified two mm. flexores minimi in digit IV in some ranoids, the ventral muscle actually being the $\mathrm{m}$. contrahentis digiti IV.
Finally, the $\mathrm{m}$. contrahentis of digit $\mathrm{V}$ is modified in Neobatrachia, acquiring a more significant component of adduction. This fact might have led it to be identified as an unrelated muscle named the $\mathrm{m}$. adductor proprius digiti $\mathrm{V}$ (see further discussion in next section).

Distinctly, in an article that was overlooked by most studies of anuran hand musculature, Ribbing (1907) identified the $\mathrm{mm}$. contrahentes as a group, describing their presence in the four digits of Discoglossus (a non-neobatrachian) and digits II, IV, and V in the neobatrachian Pelophylax. Abdala and Diogo (2010: 32) and Diogo et al. (2018: 454; but see comments in appendix 3) followed Ribbing's (1907) concept of the $\mathrm{mm}$. contrahentes, as do we.

Hand M. contrahentis digiti V: Functional Changes across Anura Obscure its Identity

Abdala and Diogo (2010: 32) argued that the $\mathrm{m}$. adductor proprius digiti V of Gaupp (1896), a name widely employed in the literature (see appendix 3), might be the $\mathrm{m}$. contrahentis of other tetrapods. We follow this hypothesis here and discuss briefly the variation of this muscle within Anura, which is relevant for identifying it as part of the $\mathrm{mm}$. contrahentes digitorum group.

The origin of the $\mathrm{m}$. contrahentis digiti $\mathrm{V}$ is similar to those of the mm. contrahentes digitorum II-IV. The mm. contrahentes usually have an adjacent or superimposed origin from the mediodistal region of the distal carpals (i.e., from the medial portion of distal carpal 3-4-5 or from the medialmost distal carpals if they are unfused), usually at the level of metacarpal III. The tendons of insertion of the $\mathrm{mm}$. contrahentes II-IV converge with those of the $m$. flexor minimus of each respective digit on the basal phalanx, with an adjacent or common insertion.

However, the insertion of the $\mathrm{m}$. contrahentis digiti $\mathrm{V}$ frequently deviates from the general morphology of the remaining $\mathrm{mm}$. contrahentes. In most anurans, the $m$. contrahentis digiti $\mathrm{V}$ inserts on the lateropalmar surface of metacarpal V (Burton, 1996, 1998a; this study). Interestingly, we found up to three distinct, 
sometimes co-occurring, points of insertion in the early diverging clades of Anura: (1) medially on the distal and medial region of the palmar surface of metacarpal $\mathrm{V}$, a fleshy insertion just medial to the $\mathrm{m}$. flexor minimus digiti $\mathrm{V}$ (pl. 8A-B); (2) intermediary, inserting on the basal phalanx of digit $\mathrm{V}$ via a tendon that either converges or is shared with that of the $\mathrm{m}$. flexor minimus digiti $\mathrm{V}$ (pl, 8C); and (3) laterally on the lateropalmar region of metacarpal $\mathrm{V}$, with a fleshy insertion lateral to the $\mathrm{m}$. flexor minimus digiti $\mathrm{V}$ (pls. 2C, 8A-B, 9B); this lateropalmar insertion is present in almost all Acosmanura (Burton, 1996, 1998a; this study).

We observed the insertion on the basal phalanx, as in plate $8 \mathrm{C}$ of Heleophryne orientalis, in Ascaphidae, Leiopelmatidae, some Costata, Rhinophrynidae (polymorphic), and Sooglossidae. The presence of the insertion on the basal phalanx (as in the mm. contrahentes digitorum IIIV) in these clades supports the correspondence of the $\mathrm{m}$. adductor proprius digiti $\mathrm{V}$ to the $\mathrm{mm}$, contrahentes group, as suggested by Abdala and Diogo (2010). A switch of the insertion to the distal and lateropalmar portion of metacarpal V (or the loss of the medial and intermediate insertion) might have occurred in Acosmanura (except for Sooglossidae and Heleophryne orientalis, in which only an intermediate insertion on the basal phalanx is present). Based on the position, connection, and direction of the muscle, this transformation of the insertion point to the lateropalmar region of the metacarpal results in greater adduction than flexion, as opposed to an insertion on basal phalanx with the $\mathrm{m}$. flexor minimus of digit $\mathrm{V}$ (in which case it mainly flexes the basal phalanx). As discussed above, this change in function and morphology might have led it to be considered an adductor muscle unrelated to the $\mathrm{mm}$. contrahentes.

Is the M. adductor pollicis a Distal Differentiation of the $\mathrm{M}$. pronator quadratus?

According to Abdala and Diogo (2010: 12), Diogo and Abdala (2010:381), and Diogo et al. (2018: 454), the $\mathrm{m}$. adductor pollicis of anurans probably corresponds to the $\mathrm{m}$. contrahentis of other tetrapods. Diogo and Ziermann (2014: 92 and table 2) state that the $\mathrm{m}$. adductor pollicis of anurans might correspond to part or the totality of the group of the mm. contrahentes, although they do not discard the possibility of it being part of the $\mathrm{m}$. pronator quadratus instead.

Consistent with the latter possibility, the $\mathrm{m}$. adductor pollicis in the early diverging clades of Anura is almost completely undifferentiated from the $\mathrm{m}$. pronator quadratus (pl. 9A, B, D). This is the case for Ascaphidae, Leiopelmatidae, Costata, Rhinophrynidae, and part of Anomocoela (the $\mathrm{m}$. adductor pollicis is apparently absent in Pipidae, in which there is no muscular portion from the distal carpals to the prepollex; see pl. 9C). In those clades, the orientation of the fibers of the muscle identified as $\mathrm{m}$. adductor pollicis (origin from the distal carpals) and $\mathrm{m}$. pronator quadratus (origin from the ulnare and distal portion of the radioulna) form an almost uniform sheet of parallel fibers attaching to the prepollex. This morphological configuration suggests that the portion from the distal carpals (identified as $\mathrm{m}$. adductor pollicis) and that from the ulnare + radioulna (identified as the $\mathrm{m}$. pronator quadratus) are derived from the same muscle (i.e., the portion from the distal carpals being a distal differentiation of the $\mathrm{m}$. pronator quadratus), as hypothesized by Diogo and Ziermann (2014).

In the apomorphic condition, the portion from the carpals is slightly differentiated from the $\mathrm{m}$. pronator quadratus in some anomocoelans, such as Megophryidae (pl. 10A), and well differentiated in Neobatrachia (e.g., Gaupp, 1896: figs. 89, 92-93; Burton, 1998a: fig. 1; pls. $2 \mathrm{D}, 10 \mathrm{~B})$. In the latter clade, the m. adductor pollicis acquires a more distal origin from the carpals, originating from the medial portion of the distal carpals (usually from the medial region of distal carpal 3-4-5) and inserts ventrad and distad to the $m$. pronator quadratus on the prepollex. This configuration determines notable differences in fiber orientation between the $m$. adductor pollicis and the $\mathrm{m}$. pronator quadratus. In this case, the position, connection, and direc- 
tion suggest that the $\mathrm{m}$. adductor pollicis generates some degree of adduction of the prepollex. This function is concordant with the "adductor" name given to this muscle by Ecker (1864) and Gaupp (1896), who only studied exemplars of Ranidae (Neobatrachia). In light of the controversies regarding the homology of this muscle, we continue using the name $\mathrm{m}$. adductor pollicis as a working hypothesis but recognize that it might not be homologous with the homonym muscle of other tetrapods (Diogo and Ziermann, 2014: 102).

Similarly, the $\mathrm{m}$. adductor pollicis and the medial $\mathrm{m}$. flexor indicis brevis profundus are morphologically similar, particularly in early diverging clades (pls. 9A, B, D, 10A) and to a lesser degree in Neobatrachia (pls. 2D, 10B). In the non-neobatrachian clades, a partially undifferentiated muscular mass radiates from the distal carpals, diverging only distally into distinct muscles as they reach their respective insertion points $(\mathrm{m}$. adductor pollicis on the prepollex, and the medial $\mathrm{m}$. flexor indicis brevis profundus on metacarpal II). This curious morphological configuration provides additional evidence that the medial $\mathrm{m}$. flexor indicis brevis profundus might be part of the same group as the $\mathrm{m}$. adductor pollicis (which might, in turn, be part of the $\mathrm{mm}$. contrahentes digitorum group or a differentiation of the $m$. pronator quadratus). Additionally, since the $\mathrm{m}$. adductor pollicis and medial $\mathrm{m}$. flexor indicis brevis profundus are proximally undifferentiated in some early diverging clades (see pl. 9A, B, D), the correspondence of the former to the $\mathrm{mm}$. flexores breves profundi cannot be discarded. The variation, identity, and homology of the medial $\mathrm{m}$. flexor indicis brevis profundus are further discussed below.

Homology of the M. flexor brevis profundus of Digit II (Index)

In digit II of Ascaphus and Leiopelma we observed a muscle that is clearly part of the $\mathrm{mm}$. flexores breves profundi group ( $\mathrm{pl}$. 9A). This unreported muscle has a similar morphology and origin as the $\mathrm{mm}$. flexores breves profundi of the remaining digits. It originates from the distal carpals, adjacent to the $\mathrm{mm}$. flexores breves profundi of digits III and IV, and has a fleshy insertion on the lateropalmar surface of metacarpal II (like the $\mathrm{mm}$. flexores breves profundi of digits III and IV). Of special relevance is that this $\mathrm{m}$. flexor brevis profundus co-occurs with the $\mathrm{m}$. opponens indicis of classical terminology.

According to Abdala and Diogo (2010), Diogo and Abdala (2010), Diogo and Ziermann (2014), and Diogo et al. (2018), the m. opponens indicis is probably part of the $\mathrm{mm}$. flexores breves profundi group. In contrast, on the basis of innervation and relative layer position Gaupp (1895: 199-200) considered the m. opponens indicis not to be part his $\mathrm{mm}$. flexores ossium metacarpi III and IV (our mm. flexores breves profundi). Nevertheless, we tentatively follow the suggestion of Diogo and associates and consider the $\mathbf{m}$. opponens indicis of classical terminology to be the medial (radial) muscle of the $\mathrm{m}$. flexor brevis profundus of digit $\mathrm{II}$, resulting in the name "medial $\mathrm{m}$. flexor indicis brevis profundus." However, the identity of this medial muscle requires further study (e.g., assessment of taxonomic variation on its innervation, development, more detailed account of its topological variation), particularly in relation to the $\mathrm{m}$. adductor pollicis, $\mathrm{m}$. contrahentis, and $\mathrm{m}$. pronator quadratus, as discussed above.

A medial (radial) and lateral (ulnar) muscle of the $\mathrm{m}$. flexor brevis profundus is present in the second digit of Caudata (Francis, 1934; Walthall and Ashley-Ross, 2006; Diogo et al., 2018), considered to be digit II (see Fabrezi and Alberch, 1996) of five-fingered tetrapods. If the lateral muscle of the $\mathrm{m}$. flexor indicis brevis profundus is plesiomorphically present in digit II of Anura, then its loss is a putative synapomorphy of Lalagobatrachia (sister taxon of Ascaphus + Leiopelma). Nevertheless, this hypothesis is contingent on three points. First, there is controversy regarding the digit-to-digit correspondence between anurans and caudates (e.g., Diogo and Ziermann, 2014). Second, detailed studies describing the palmar musculature of cryptobranchoid caudates are 
lacking (the descriptions of Humphry, 1972: 40, for Andrias, and Ribbing, 1907: 634, for Cryptobranchus, are not detailed enough to understand the condition of this muscle). Third, the musculature of the most radial (preaxial) and ulnar (postaxial) digits might be highly modified to adopt some specific functions required on these digits, such as adduction and abduction, obscuring the identities (i.e., muscles of the most radial digit of Caudata are expected to be more similar to the most radial digit of Anura, despite being the nonhomologous digits I and II, respectively; see Abdala and Diogo, 2010, and "M. abductor pollicis longus and attachments: position versus digit identity," below).

Hand Mm. flexores breves profundi and flexores digitorum minimi Groups

The $\mathrm{mm}$. flexores breves profundi are usually composed of a single muscle in digits II-V. In digit II, only Ascaphus and Leiopelma have two muscles (see previous section and pl.9A), while in digit $\mathrm{V}$ the $\mathrm{m}$. flexor brevis profundus may be absent in some species (Burton, 1998a; this study; see pl. 2D as example of its absence).

The number of muscles that comprise the $\mathrm{mm}$. flexores breves profundi of Anura is confusing in the literature. On the basis of our analysis of the studies that employed the term $\mathrm{mm}$. flexores breves profundi, we conclude that most differences or inconsistencies are the result of the inclusion or exclusion of the $\mathrm{mm}$. flexores digitorum minimi within it. The inclusion of the latter group as part of the $\mathrm{mm}$. flexores breves profundi might have led some authors to consider the presence of eight muscles, composed of a medial (radial) and a lateral (ulnar) muscle in each digit (the medial usually corresponding to the mm. flexores digitorum minimi); this hypothesis is justified and discussed in chronological order below.

Although McMurrich (1903a, 1903b) did not study any anurans, he described the $\mathrm{mm}$. flexores breves profundi in his amphibian sample, the caudate Ambystoma, in the context of a comparative study of the forearm flexor muscles of tetra- pods. McMurrich (1903b: 465) described the medial and lateral muscles of the $\mathrm{mm}$. flexores breves profundi that originate on the carpals (corresponding to our concept of this muscle group) and additional muscles that originate on the metacarpals. These latter muscles correspond to our $\mathrm{mm}$. flexores digitorum minimi, a relation that highlights a historical association between both groups of muscles.

Later, Ribbing (1907) described the mm. flexores breves profundi (p. 635) and flexores digitorum minimi (p. 643) as distinct muscle groups, corresponding entirely or almost entirely to our concepts and names. However, in his figure 13 of Discoglossus and figure 14 of Pelophylax, the mm. flexores breves profundi are depicted as having two muscles on digits III-IV and digits III-V, respectively. The medial muscle of digits III-V depicted in both figures corresponds to the description of the $\mathrm{mm}$. flexores digitorum minimi of Ribbing (1907: 643), which might have been a labeling mistake or, alternatively, an implicit (i.e., not stated) higher hierarchic level of arrangement for both groups of muscles, resulting in a more inclusive group of $\mathrm{mm}$. flexores breves profundi sensu lato (i.e., including the $\mathrm{mm}$. flexores digitorum minimi).

Our conjecture that Ribbing (1907) either mislabeled or implicitly grouped the $\mathrm{mm}$. flexores digitorum minimi under the $\mathrm{mm}$. flexores breves profundi is supported by our current knowledge of the topological variation of the $\mathrm{mm}$. flexores minimi of digits III and IV across Anura (Gaupp, 1896; Burton, 1998a, 1998b; char. 9). First, it can be interpreted from his figure 13 of Discoglossus that the medial muscles of digits III and IV have a more distal origin (apparently from the metacarpals, which is a characteristic of the mm. flexores minimi; illustrations are not detailed enough to be conclusive about the exact point of origin) ventral to their corresponding $\mathrm{m}$. intermetacarpalis. Second, Pelophylax is figured with the origins of the medial muscles of digits III and IV dorsal to their corresponding $\mathrm{m}$. intermetacarpalis. These descriptions are fully congruent with the $\mathrm{mm}$. 
flexores minimi of digits III and IV in Discoglossus and Pelophylax. The mm. flexores minimi of digits III and IV of D. pictus have a ventral origin (or position) relative to the $\mathrm{mm}$. intermetacarpales I and II, respectively (Burton, 1998 b; this study), whereas the mm. flexores minimi of digits III and IV of Pelophylax have a dorsal origin relative to the $\mathrm{mm}$. intermetacarpales I and II (see Gaupp, 1896: fig, 94).

The interpretation of the mm. flexores minimi of digits III-V as the medial muscles of the $\mathrm{mm}$. flexores breves profundi, as shown in Ribbing's (1907) figures was apparently accepted and replicated by Diogo and associates, although with some inconsistencies. Abdala and Diogo (2010: 33 ) and Diogo and Abdala (2010: 382-383) considered that the $\mathrm{mm}$. flexores digitorum minimi probably correspond to/derive from the $\mathrm{mm}$. flexores breves profundi and described their $\mathrm{mm}$. flexores breves profundi as being composed of eight muscles. Considering this, the medial muscles of digits III-V are our $\mathrm{mm}$. flexores digitorum minimi, while the lateral muscles are our $\mathrm{mm}$. flexores breves profundi.

The identities of the two muscles of the $\mathrm{m}$. flexor brevis profundus described by Abdala and Diogo (2010) and Diogo and Abdala (2010) as occurring in digit II are unclear. The only published record of an $\mathrm{m}$. flexor minimus in digit II is in the overlooked study of Ribbing (1911) in Pipa cf. pipa (corroborated here for our Pipa pipa specimen and also observed in a few additional species; see char. 8). Furthermore, digit II is characterized by having a single $\mathrm{m}$. flexor brevis profundi (the medial one), with the only exception of Ascaphus and Leiopelma (pl. 9A), species not studied by them.

Diogo and Ziermann (2014), unlike Abdala and Diogo (2010) and Diogo and Abdala (2010), recognized the $\mathrm{mm}$. flexores digitorum minimi as distinct from the $\mathrm{mm}$. flexores breves profundi (their table 2). However, Diogo and Ziermann (2014: 92-93) also described eight muscles of the mm. flexores breves profundi in Elcutherodactylus coqui, with a medial and lateral muscle in each digit. The $\mathrm{mm}$. flexores breves profundi are not clearly defined or visible in their figures. Additionally, Diogo and Ziermann (2014: 93) stated that they "could not find (the mm. flexores digitorum minimi) in the adults and froglets examined, either because they are very deep and/ or deeply blended with other hand muscles, or because they are absent." Our interpretation is that Diogo and Ziermann (2014) probably considered the $\mathrm{mm}$. flexores digitorum minimi to be the medial muscles of the mm. flexores breves profundi in digits III-V, since the $\mathrm{mm}$. flexores digitorum minimi of digits III-V are present and well developed in this species (Burton, 1998a, 1998b; pl. 10B). Furthermore, of the two muscles of the $\mathrm{mm}$. flexores breves profundi reported to occur in digit II, only the medial one is clearly described, and it corresponds to our medial $\mathrm{m}$. flexor indicis brevis profundus (see pl. 10B).

Diogo et al. (2018: 455-456; see also Diogo and Ziermann, 2014), considered the mm. flexores digitorum minimi to be a distinct group, and they reported them separately from the $\mathrm{mm}$. flexores breves profundi in their table 18.1 (p. 456). However, they continued listing eight muscles of the $\mathrm{mm}$. flexores breves profundi in this table (p. 455). Given that the book by Diogo et al. (2018) is partially a compilation and update of their previous contributions (e.g., Abdala and Diogo, 2010; Diogo and Abdala, 2010; Diogo and Ziermann, 2014), a possible explanation is that the text in their table 18.1 was not updated when referring to the $\mathrm{mm}$. flexores breves profundi. In this case, from the point in which the $\mathrm{mm}$. flexores digitorum minimi are considered to be a distinct muscle group, the $\mathrm{mm}$. flexores breves profundi need to be reinterpreted as being composed of a single lateral muscle, at least in digits III-V (where the $\mathrm{mm}$. flexores digitorum minimi are almost invariably present across Anura).

Finally, the identity of the lateral muscle of the two $\mathrm{mm}$. flexores breves profundi of digit II reported by Diogo et al. (2018) is not clear. The species explicitly studied by them is Lithobates pipiens (Ranidae), which we did not study but predict to have a single $\mathrm{m}$. flexor brevis profundus (the medial muscle) and lack the $\mathrm{m}$. flexor 
minimus indicis, given that medial and lateral $\mathrm{mm}$. flexores breves profundi were observed only in Ascaphus and Leiopelma (see section above), while an $\mathrm{m}$. flexor minimus indicis (which might have been considered an $\mathrm{m}$. flexor brevis profundus) is extremely uncommon in Neobatrachia (see char. 8).

Homology and Identity of the M. contrahentium caput longum in Anura

Ribbing (1907; 625-626; 1911: 13) considered his anuran $\mathrm{m}$. caput longum muscularum contrahentium to correspond to the $\mathrm{m}$. intercarpalis and m. ulnocarpalis of Gaupp (1896), hypothesizing that it is divided into a proximal and distal portion in Anura. Abdala and Diogo (2010: 9), Diogo and Abdala (2010: 375), Diogo and Ziermann (2014: table 2), and Diogo et al. (2018: 447) followed this hypothesis, employing a slightly modified name: $m$. contrahentium caput longum.

We tentatively follow the hypotheses of Ribbing (1907) and Diogo and associates, adding the terms "distalis" and "proximalis" to distinguish between the $\mathrm{m}$. intercarpalis and $\mathrm{m}$. ulnocarpalis of classical terminology, resulting in the names $m$. contrahentis caput longus distalis and $\mathrm{m}$. contrahentis caput longus proximalis, respectively. The $\mathrm{m}$. contrahentis caput longus distalis originates from the ulnare and inserts on the distal carpals (char. 10; pls. 2D, 9A-C, 10B), while the m. contrahentis caput longus proximalis originates from the radioulna and inserts on the ulnare (e.g., Gaupp, 1896; Ribbing, 1907; pls. 2D, 9A, B).

The fiber orientation and connections observed in Ascaphidae + Leiopelmatidae and Costata (Ribbing 1907: fig. 11; pl. 9A, B) might support the division of the $\mathrm{m}$. contrahentium caput longum into a distal and proximal portion in Anura. In those clades, the $\mathrm{mm}$. contrahentis caput longus distalis and proximalis have similar fiber orientation, and some fibers of the former arise from the tendon of insertion of the $\mathrm{m}$. contrahentis caput longus proximalis as it attaches to the ulnare (Ribbing, 1907: 626; pl. 9A, B). As a derived condition, more markedly expressed in Neobatrachia, the distal portion acquires a more oblique orientation with respect to the proximal portion, and the tendinous connection is partially or completely lost (pl. 2D). Furthermore, and also possibly supporting a subsequent differentiation into a proximal and distal portion in Anura, both portions are innervated by the same nerve in, at least, Pelophylax cf. esculenta (see Gaupp, 1899: 179-180, fig. 52).

Finally, under the name of $\mathrm{m}$. contrahentium caput longum, Diogo and Ziermann (2014: 91) described for Eleutherodactylus coqui a muscle that "runs from the distal radio-ulna to the ulnare." This description corresponds to our $\mathrm{m}$. contrahentis caput longus proximalis ( $\mathrm{m}$. ulnocarpalis of classical terminology). They did not describe a muscle running from the ulnare to the distal carpals, which corresponds to our m. contrahentis caput longus distalis ( $\mathrm{m}$. intercarpalis of classical terminology). However, a typical m. contrahentis caput longus distalis is present in the specimen of $E$. coqui examined by us (pl. 10B).

M. abductor pollicis longus and Attachments: Position versus Digit Identity

The first question to be raised here is the change of the name from classical anuran terminology ( $\mathrm{m}$. abductor indicis longus) to the new proposal of Diogo and associates ( $\mathrm{m}$. abductor pollicis longus). This implies both that Anura lacks digit I (the pollex, which results in the term pollicis of the muscle name of the new proposal) and that this muscle is present and inserts on digit II (the index, which results in the term indicis of the classical muscle name) instead.

Accordingly, Abdala and Diogo (2010: 34) and Diogo et al. (2015: 526) suggested that the $\mathrm{m}$. abductor pollicis longus attaches to the radialmost digit, and since anurans are considered to have lost digit $I$, the attachment is instead to digit II. Possibly supporting this heterotopic shift, the m. abductor pollicis longus in Ascaphidae + Leiopelmatidae inserts on the prepollex (this study), with a shift in the attachment to digit II in its sister clade, Lalagobatrachia. However, the direction of change is unclear, since the insertion on the prepollex in Ascaphidae + Leiopelmatidae could 
be the novelty instead. Although the prepollex is absent in caudates, a well-defined transformation series including the relevant variation in Caudata for comparison would enable the hypothesis that the attachment occurs on the radialmost element to be tested.

Homology of the Foot M. flexor brevis profundus of Digit I

Diogo and Ziermann (2014) introduced the name $\mathrm{m}$. flexor hallucis accessorius for the $\mathrm{m}$. opponens hallucis of classical terminology. Diogo and Molnar (2014: table 8) and Diogo et al. $(2018: 507,579,585)$ discussed the identity of the $\mathrm{m}$. flexor hallucis accessorius of anurans, considering that it might be derived from the $\mathrm{mm}$. contrahentes (as suggested by Dunlap, 1960 ) or from the $\mathrm{mm}$. flexores breves profundi (as suggested by Perrin, 1892). However, Perrin (1892) associated the $\mathrm{m}$. opponens hallucis with the $\mathrm{mm}$. flexores digitorum minimi and not with the $\mathrm{mm}$. flexores breves profundi. Alternatively, Ribbing $(1909,1911)$ associated the $m$. flexor hallucis accessorius with the mm. flexores breves profundi group. We discuss below the putative relationship of $\mathrm{m}$. flexor hallucis accessorius with other muscle groups.

We found in Ascaphus a muscle attached to digit I that clearly corresponds to the $\mathrm{mm}$. flexores breves profundi (named here $\mathrm{m}$. flexor hallucis brevis profundus) and co-occurs with the $\mathrm{m}$. flexor hallucis accessorius, as shown in plate $11 \mathrm{~B}$. The presence of the $\mathrm{m}$. flexor hallucis brevis profundus might vary intraspecifically in $\mathrm{Asca}$ phus, since a muscle with these characteristics was not described or figured by Dunlap (1960).

The problem that remains is whether the $\mathrm{m}$. flexor hallucis accessorius corresponds to the medial/tibial muscle of the $\mathrm{mm}$. flexores breves profundi of digit I, in a curious analogy with the same muscle group of the hand. However, contrary to the hand, there is evidence that the $\mathrm{m}$. flexor hallucis accessorius is a differentiated portion of the $\mathrm{m}$. contrahentis pedis hallucis, given that there are species in which it is difficult to differentiate both muscles (Dunlap,
1960: 41; this study; see also their complementary morphological configuration in pl. 4D). On this basis, and considering that a muscle that clearly corresponds to the mm. flexores breves profundi was identified here in Ascaphus, we follow Diogo and Ziermann (2014) in referring to the m. opponens hallucis of classical terminology as the $\mathrm{m}$. flexor hallucis accessorius, and not as the medial muscle of the $\mathrm{m}$. flexor brevis profundus of digit I.

Foot Mm. abductores breves dorsales of Digits II and III: Recurrent Simultaneous Origin of Novelties in Adjacent Digits

These previously unreported muscles are considered here to form their own muscle group, together with the $\mathrm{m}$. abductor brevis dorsalis hallucis. They share notably similar morphologies, attachments, functions, and position relative to other muscles; below we discuss the basis for this grouping.

The $\mathrm{m}$. abductor brevis dorsalis hallucis originates on the prehallux and/or element Y. It has a fleshy insertion on the dorsomedial surface of metatarsal I, producing abduction of digit I (Gaupp, 1896; Dunlap, 1960; Burton, 2004; this study; pls. $5 \mathrm{D}, 12 \mathrm{C}, 14)$. In most species a slip of the medial $\mathrm{m}$. dorsometatarsalis hallucis proximalis originates on the same region on the prehallux and/or element $\mathrm{Y}$, resulting in proximal fusion or a common origin in some species (Burton, 1983; this study). This is precisely the morphology observed in the $\mathrm{mm}$. abductores breves dorsales digitorum II and III, with the difference that their origins are located on the proximal end of metatarsals I and II, respectively (see chars. 19 and 20; pl. 14), and they abduct metatarsals II and III, respectively.

The taxonomic distribution of the presence of these two muscles is striking. Both muscles evolved simultaneously and independently in some Megophryidae, Sooglossidae, Laurentobatrachia, and Neobatrachus. Leptobrachella bourreti (Megophryidae) is the only species of these clades in which one of these two muscles is missing (although this observation requires corroboration to discard a false negative). This 
pattern of convergences is further discussed in the section "Origin of novelties in the anuran hand and foot musculature."

Since the mm. abductores breves dorsales digitorum II and III connect the metatarsals, it might be argued that they are portions of the $\mathrm{mm}$. intermetatarsales. However, we reject this hypothesis because (1) they co-occur with typical $\mathrm{mm}$. intermetatarsales; (2) their origins are restricted to the proximal end of the metatarsals (the $\mathrm{mm}$. intermetatarsales usually have broad origins and insertions, resulting in more transversal muscles); and (3) their position is more dorsally located than the $\mathrm{mm}$. intermetatarsales.

The Mm. flexores minimi of Digit I of the Foot (Hallux) and Digit II of the Hand (Index)

An m. flexor minimus occurring on digit II of Anura was first reported as such by Ribbing (1911) in Pipa cf. pipa. We corroborated the presence of this muscle in our sample of Pipa pipa (it is absent in the other congeneric species) and other species of different clades (see char. 8). The morphology of this muscle is identical to that of the $\mathrm{mm}$. flexores minimi digitorum III-V, being a deep muscle characterized by a fleshy origin from metacarpal II and a proximal insertion on the palmar surface of the basal phalanx, in common or adjacent to the insertion of the $\mathrm{m}$. contrahentis indicis (pl. 8C). Similarly to the hand, Burton (2004: 210) found a true $\mathrm{m}$. flexor teres in the hallux ("true" because it was previously confused with the $\mathrm{m}$. contrahentis pedis), opening the question of which name should be applied to this "flexor teres" in the new terminology.

Following the terminology of Diogo and associates, particularly Diogo and Ziermann (2014) and Diogo et al. (2018), the mm. flexores teretes of classical terminology are their $\mathrm{mm}$. flexores digitorum minimi of the hand and foot (but see confusion with the hand $\mathrm{mm}$. flexores breves profundi discussed above). We adopt their terminology here, which results in the configuration of the terms mm. flexor minimus hallucis and flexor minimus indicis, in reference to the muscle of digit I of the foot and digit II of the hand, respectively.
Mm. dorsometacarpales and dorsometatarsales: Recurrent Muscular Differentiation as Novelties?

Although without assigning a new name, Dunlap (1960) first described the $\mathrm{mm}$. dorsometatarsales distales of the foot as a morphological variant of the $\mathrm{mm}$. dorsometatarsales proximales (see details in appendix 3 ). These muscles were later defined as a distinct muscle group by Burton (1996) for the hand and subsequently by Faivovich (2002) for the foot (both under the name of $\mathrm{mm}$. extensores breves distales). The muscles of the hand were considered to be adaptations for arboreal life by Burton (1998c), and we extend this hypothesis to the muscles of the foot on the same grounds he cited.

The $\mathrm{mm}$. dorsometacarpales/dorsometatarsales distales have a fleshy origin on the latero- and mediodistal surface of the metacarpals/metatarsals (a few species also have additional origins on the phalanges; this study). Their fibers extend along the phalanges (the length of the fleshy portion varies) and insert via a tendon on the distal interphalangeal joints and distal phalanges (Burton, 1996: figs. 2-3; 1998c: fig. 1; 2004: fig. 2; pls. 3 and 5). Burton (1996) suggested that the $\mathrm{mm}$. dorsometacarpales distales might have evolved from the $\mathrm{mm}$. dorsometacarpales proximales. We found substantial evidence supporting this hypothesis and further extend it to the mm. dorsometatarsales distales (in which case they derive from the $\mathrm{mm}$. dorsometatarsales proximales). Supporting this hypothesis, some species lack a gap or different orientation of the fibers between the $\mathrm{mm}$. dorsometacarpales/ dorsometatarsales distales and the $\mathrm{mm}$. dorsometacarpales/dorsometatarsales proximales, forming a continuous origin from the proximal region of the metacarpal/metatarsal to the distal portion of this bone (this study). Additionally, the fibers of the $\mathrm{mm}$. dorsometacarpales/dorsometatarsales proximales reach the phalanges in some species, which is characteristic of the $\mathrm{mm}$. dorsometacarpales/ dorsometatarsales distales (Burton, 1998a, 1998c, 2004). These two facts suggest that a partial differentiation of the $\mathrm{mm}$. dorsometacarpales/dorso- 
metatarsales proximales into the $\mathrm{mm}$. dorsometacarpales/dorsometatarsales distales occurs in some species.

Furthermore, there is a great variation in the number of tendons of insertion and points of insertion in the $\mathrm{mm}$. dorsometacarpales/dorsometatarsales proximales. These muscles usually insert via a tendon on the distal interphalangeal joint/distal phalanx and frequently (in the same species and some digits) have additional tendons to more proximal interphalangeal joints and/or metacarpophalangeal/ metatarsophalangeal joints (this study; see also Dunlap, 1960; Burton, 2004). In several species, we observed that when the $\mathrm{m}$. dorsometacarpalis/dorsometatarsalis distalis (which invariably inserts on the distal interphalangeal joint/distal phalanx) is present, the $\mathrm{m}$. dorsometacarpalis/dorsometatarsalis proximalis inserts on more proximal interphalangeal joints instead of the distal one. In this specific case, the fleshy portion with the tendon of insertion on the distal interphalangeal joint/distal phalanx might have differentiated into what is termed an $\mathrm{m}$. dorsometacarpalis/dorsometatarsalis distalis (i.e., the portion with latero- or mediodistal origin on the metacarpal/metatarsal), while the $\mathrm{m}$. dorsometacarpalis/dorsometatarsalis proximalis (with more proximal origin) inserts on a more proximal joint.

The fact mentioned above of the multiple and changing points of insertions of the $\mathrm{mm}$. dorsometacarpales/dorsometatarsales proximales leads to a question to be further investigated: does the presence of $\mathrm{m}$. dorsometacarpalis/dorsometatarsalis distalis relax functional constraints on the $\mathrm{m}$. dorsometacarpalis/dorsometatarsalis proximalis, enabling its insertion point to vary? The four digits of the hand and the five of the foot have these muscle groups, allowing a robust test of this hypothesis in the several unrelated arboreal anuran clades in which they occur.

\section{Origin of Novelties in the Anuran Hand ANd Foot Musculature}

This section briefly addresses the origin of novelties-by which we mean apomorphic structures (muscles and slips) - in anuran hand and foot mus- culature (for discussions of competing definitions of evolutionary novelty, see Moczek, 2008; Pigliucci, 2008; Peterson and Müller, 2013). Specifically, we are interested in (1) distinguishing between apomorphic structures that arose de novo without a plesiomorphic antecedent and apomorphic structures that are transformations of plesiomorphic structures (i.e., between Type II and Type III novelties of Müller, 2010), and, in the latter case, (2) identifying the classes of transformations (e.g., divisions, topological rearrangements) that gave rise to the new structures. This account is presented in a narrative form (i.e., without quantitative analysis); as such, it should be considered a preliminary approximation whose main objective is to suggest possible paths for future studies and potential tests of the hypotheses of transformations.

\section{Muscle Splitting without Topological Changes}

Examples of splitting of preexisting muscles without modifications in the position of the muscle occur in the large palmar and plantar flexors: the $\mathrm{m}$. flexor digitorum communis and the $\mathrm{mm}$. flexores breves superficiales, respectively. Both are plesiomorphically single muscles with a single tendon of insertion, as exemplified for the palmar $\mathrm{m}$. flexor digitorum communis in plate 6A-D. Plesiomorphically and in nonarboreal clades, these palmar and plantar flexors attach indirectly to the distal phalanges of all or the more ulnar/fibular (postaxial) digits through either the flexor plate in the hand or by a contribution of the aponeurosis plantaris in the foot (e.g., Dunlap, 1960; Burton, 1996, 1998a, 2004; pl. 6A-D).

The tendon of insertion of the palmar $\mathrm{m}$. flexor digitorum communis is divided in arboreal clades, which is accompanied by different degrees of muscular differentiation into discrete slips (e.g., pl. 2B; Burton, 1996, 1998a; this study). In some species, the muscular splitting is complete, resulting in two or three almost totally independent slips from origin to insertion.

Whereas the palmar flexor is divided simultaneously with the origin of arboreality in each anuran clade, the division of the plantar $\mathrm{mm}$. 
flexores breves superficiales deviates somewhat from this pattern (Burton, 2004; this study). There are species of arboreal clades with the plantar muscle undivided, and a detailed study with broader taxon sampling is required at this point to establish if its division occurs simultaneously with the origin of the arboreality in each clade. Nevertheless, the division of the plantar $\mathrm{mm}$, flexores breves superficiales only occurs in or within arboreal clades, suggesting that, like the division of the palmar flexor, it is a novelty related to climbing.

\section{Muscle Splitting with Topological Changes}

Three examples from the palmar surface and a fourth example involving equivalent muscles of the dorsal surfaces of the hand and the foot involve muscle splitting accompanied by changes in the origin, insertion, and/or fiber orientation of the muscles. The first case refers to the $\mathrm{m}$. flexor indicis superficialis proprius and $\mathrm{m}$. caput profundum digiti III, which are hypothesized to derive from slips differentiated from the $\mathrm{m}$. flexor accessorius. In this case we hypothesize that the distal portion of the m. flexor accessorius with origin on the distal carpals and insertion on the dorsal surface of the flexor plate at level of the bases of the tendines superficiales of digits II and III (plesiomorphically observed in early diverging clades) were distally developed as a derived condition, forming discrete and independent muscular bodies in those digits. These modifications resulted in the $\mathrm{m}$. flexor indicis superficialis proprius and $\mathrm{m}$. caput profundum digiti III (see further comments in the section, above, $M$. flexor indicis superficialis proprius, $m$. caput profundum digiti III, and $\mathrm{m}$. flexor accessorius: Novelties from Splitting?).

The second example refers to the $\mathrm{m}$. adductor pollicis, which probably evolved as a distal differentiation of the $\mathrm{m}$. pronator quadratus, resulting in a moderate topological modification but a clear functional change. We suggest that the distal portion of the large and single muscle with a continuous origin on the radioulna, ulnare, and distal carpals observed in early diverging clades were differentiated into a proximal portion from the radioulna and ulnare ( $\mathrm{m}$. pronator quadratus) and a distal portion from the distal carpals (m. adductor pollicis), with a markedly change in fiber orientation (see further comments in section, above, Is the M. adductor pollicis a Distal Differentiation of the $\mathrm{m}$. pronator quadratus?").

The third example is similar to the second one and refers to the hypothesized division of the $m$. contrahentium caput longum (as it is present in Caudata) into a proximal and distal portion in Anura. Furthermore, a more markedly positional change of the distal portion (the $\mathrm{m}$. contrahentis caput longus distalis) is observed in Neobatrachia.

The fourth example comes from the equivalent muscles of the dorsal surface of the hand and foot: the $\mathrm{mm}$. dorsometacarpales distales and $\mathrm{mm}$. dorsometatarsales distales, respectively. These muscle groups, with the function of detaching the adhesive disc, evolved simultaneously in different digits of the hand and the foot in unrelated arboreal clades (Burton, 1998c, 2004; this study). The underlying hypothesis is that these muscles evolved as a distal differentiation and distal displacement of the $\mathrm{mm}$. dorsometacarpales proximales and $\mathrm{mm}$. dorsometatarsales proximales.

Neomorphic Elements, Replication, and/or Muscle Splitting?

De novo evolution of character states (Type II novelty) is probably the most striking kind of novelty and is usually difficult to distinguish from the differentiation of preexisting structures (e.g., Hurley et al., 2005). We find two examples here: the $\mathrm{mm}$. abductores breves dorsales of digits II and III (see chars. 19 and 20 and section, above, Foot Mm. abductores breves dorsales of Digits II and III: Recurrent Simultaneous Origin of Novelties in Adjacent Digits), and the supplementary slips of the $\mathrm{mm}$. lumbricales breves of the foot described by Blotto et al. (2017).

There are several aspects to be considered when discussing whether these novel muscles evolved by splitting of preexisting muscles by an independent process in each digit (resulting 
in identical morphologies), from independent, de novo innovation in each digit, or by splitting in one digit and subsequent replication of this developmental and genetic machinery in the adjacent digits. We briefly describe the two muscle groups and their taxonomic distribution, and then discuss alternative preliminary hypotheses.

The $\mathrm{mm}$. abductores breves dorsales of digits II and III are named as such on the basis of their morphological, topological, connective, and functional similarities with the $\mathrm{m}$. abductor brevis dorsalis of digit $\mathrm{I}$, which is plesiomorphically and almost invariably present in Anura (Dunlap, 1960; this study). The muscles of digits II and III arose together and separately in multiple, distantly related clades, including Megophryidae, Sooglossidae, Laurentobatrachia, and Neobatrachus aquilonius. The $\mathrm{m}$. abductor brevis dorsalis of digit I is plesiomorphically present in these taxa as well.

Blotto et al. (2017) described a similar situation for a set of novel, identical slips occurring on adjacent digits on the plantar surface of Macrogenioglottus + Odontophrynus (Odontophrynidae). They described supplementary slips of the $\mathrm{mm}$. lumbricales breves digitorum II, III, and IV with fleshy insertions on the ventral surface of their respective metatarsal. Although some intraspecific variation was observed, most species presented the supplementary slips of digits III and IV. Considering the ACSRs, the supplementary slips of digits III and IV arose simultaneously in the ancestor of Macrogenioglottus + Odontophrynus, whereas the slip of digit II arose within internal clades (in Macrogenioglottus and part of Odontophrynus).

The more general question underlying these two examples is whether a preexisting genetic regulatory network responsible for the development of a muscle in one digit is replicated for the development of identical muscles in adjacent digits. In the example of the $\mathrm{mm}$. abductores breves dorsales, the muscle of digit $\mathrm{I}$ is plesiomorphically present in the clades in which the muscles of digits II and III arose later. In this case, a replication of the developmental and genetic regulatory network might have given rise to the muscles of digits II and III.

Similarly, the genetic machinery that resulted in the development of the supplementary slips of digits III and/or IV (both evolved simultaneously in the ancestor of Macrogenioglottus + Odontophrynus) might have been replicated to give rise to the supplementary slip of digit II (which evolved in less inclusive clades). If the hypothesis of a replication of a novelty in adjacent digits is accepted, a main difference emerges with respect to the previous example of the $\mathrm{mm}$. abductores breves dorsales: at least one of these supplementary slips of the mm. lumbricales breves must have evolved first, since all these supplementary slips are novelties restricted to Odontophrynidae. In contrast, in the case of the $\mathrm{mm}$. abductores breves dorsales, the muscle of digit I is present plesiomorphically.

In conclusion, this account of the novelties in the anuran hand and foot musculature leaves a series of open questions. Whereas evidence for the transformations involved in the origin of some novelties is strong in some cases, multiple alternative hypotheses can be invoked to explain others. The examples presented here provide a diverse and rich source of novelties that warrant further investigation employing approaches from different fields of evolutionary biology.

\section{CONCLUSIONS}

This study shed light on the still insufficiently explored diversity of amphibians. Although this study fills important gaps in knowledge of the early diverging clades of anurans, detailed studies of the musculature of cryptobranchoid caudates are required to understand the homologies between anuran and caudate muscles and determine character polarity. Moreover, our results serve as a starting point to clarify diverse, interrelated problems, including (1) the synapomorphies for Anura and its early diverging clades; (2) muscle homology among anurans, caudates, 
and other tetrapods; (3) the implications of the hypotheses of the specific finger lost in anurans and caudates; and (4) the underlying mechanisms involved in the origin of novelties, such as the striking simultaneous evolution of identical muscles in adjacent digits.

\section{ACKNOWLEDGMENTS}

This work began during the B.L.B.'s Ph.D. studies at the Museo Argentino de Ciencias Naturales "Bernardino Rivadavia"-CONICET and was completed during a postdoctoral fellowship at the Instituto de Biociências (Universidade de São Paulo). This research was supported by grants 2012/10000-5, 2013/20423. $3,2015 / 11237-7$, and 2018/15425-0 from the São Paulo Research Foundation (FAPESP) and initially by a CONICET doctoral fellowship. B.L.B., J.F, and M.O.P. acknowledge support from PICT 404-2013, 813-2015, and 820-2015. TNT was provided free by the Willi Hennig Society. For access to collections and specimen loans we thank Lauren Scheinberg (California Academy of Sciences), Nadya Pupin and Célio F.B. Haddad (CFBH), Stephen Rogers and José Padial (Carnegie Museum of Natural History), Alan Resetar (Field Museum of Natural History), John D. Lynch (ICN), Diego Baldo (LGE), Aline Staskowian Benetti (MZUSP), Victor G. D. Orrico (Universidade Estadual de Santa Cruz), Luke Welton and Rafe Brown (University of Kansas), and Gregory Schneider and Ron Nussbaum (University of Michigan). For access to complementary specimens we thank Paula Muzzopappa. For discussions on hand and foot musculature we thank Katyuscia Araujo-Vieira and Paulo D.P. Pinheiro. For logistical help during B.L.B.'s extended visits to the University of Kansas, we are especially grateful to Rafe Brown, Luke Welton, Ashley Welton, Richard Glor, Linda Trueb, Kerry Cobb, and Wm. Leo Smith. The critical reviews of different versions of the manuscript by Virginia Abdala, Darrel Frost, and Alexander Haas greatly enhanced its scope and quality.

\section{REFERENCES}

Abdali, V., and R. Diogo. 2010. Comparative anatomy, homologies and evolution of the pectoral and forelimb musculature of tetrapods with special attention to extant limbed amphibians and reptiles. Journal of Anatomy 217: 536-573.

Abdala, V., and S. Moro. 2006. Comparative myology of the forelimb of Liolaemus sand lizards (Liolaemidae). Acta Zoologica 87: 1-12.

Abdala, V., M.L. Ponssa, M.J. Tulli, A.-C. Fabre, and A. Herrel. 2018. Frog tendon structure and its relationship with locomotor modes. Journal of Morphology 279: 895-903.

Aguilar, C., and N. Valencia. 2009. Relaciones filogenéticas entre telmatobiinidos (Anura, Ceratophryidae, Telmatobiinae) de los Andes centrales basado en la morfología de los estados larval y adultos. Revista Peruana de Biología 16: 43-50.

Alberch, P., and E.A. Gale. 1985. A developmental analysis of an evolutionary trend: digital reduction in amphibians. Evolution 39: 8-23.

Andersen, M.L. 1978. The comparative myology and osteology of the carpus and tarsus of selected anurans. Ph.D. dissertation, Department of Systematics and Ecology, University of Kansas, Lawrence.

Araujo-Vieira, K, et al. 2019. A total evidence analysis of the phylogeny of hatchet-faced treefrogs (Anura: Hylidae: Sphaenorhynchus). Cladistics 35: 469-486.

Barej, M.F., et al. 2014. The first endemic West African vertebrate family-a new anuran family highlighting the uniqueness of the Upper Guinean biodiversity hotspot. Frontiers in Zoology 11: 8 .

Barrio, A. 1968. Revisión del género Lepidobatrachus Budgett (Anura, Ceratophrynidae). Physis 27: 445-454.

Barrionuevo, J.S. 2017. Frogs at the summits: phylogeny of the Andean frogs of the genus Telmatobius (Anura, Telmatobiidae) based on phenotypic characters. Cladistics 33: 41-68.

Bewick, A.J., F.J. Chain, J. Heled, and B.J. Evans. 2012. The pipid root. Systematic Biology 61: 913-926.

Biju, S.D., and F. Bossuyt. 2003. New frog family from India reveals an ancient biogeographical link with the Seychelles. Nature 425: 711-714.

Blotto, B.L., et al. 2013. Phylogenetic relationships of a Patagonian frog radiation, the Alsodes + Eupsophus clade (Anura: Alsodidae), with comments on the supposed paraphyly of Eupsophus. Cladistics 29: 113-131.

Blotto, B.L., M.O. Pereyra, J. Faivovich, P.H.D.S. Dias, and T. Grant. 2017. Concentrated evolutionary nowelties in the foot musculature of Odontophrynidae 
(Anura: Neobatrachia), with comments on adaptations for burrowing. Zootaxa 4258: 425-442.

Bock, W.J., and C.R. Shear, 1972. A staining method for gross dissection of vertebrate muscles. Anatomischer Anzeiger 130: 222-227.

Brown, R.M., C.D. Siler, A.C. Diesmos, and A.C. Alcala. 2010 ("2009"). Philippine frogs of the genus Leptobrachium (Anura: Megophryidae); phylogeny-based species delimitation, taxonomic review, and descriptions of three new species. Herpetological Monographs 23: 1-44.

Brown, R.M., C.D. Siler, S.J. Richards, A.C. Diesmos, and D.C. Cannatella. 2015. Multilocus phylogeny and a new classification for Southeast Asian and Melanesian forest frogs (family Ceratobatrachidae). Zoological Journal of the Linnean Society 174: 130168.

Burton, T.C. 1983. The musculature of the Papuan frog Phrynomantis stictogaster (Anura: Microhylidae). Journal of Morphology 175: 307-324.

Burton, T.C. 1986. A reassessment of the Papuan subfamily Asterophryinae (Anura: Microhylidae). Records of the South Australian Museum 19: 405450.

Burton, T.C. 1996. Adaptation and phylogeny in the hand muscles of Australo-Papuan hylid frogs (Anura: Hylidae: Pelodryadinae). Australian Journal of Zoology 44: 611-623.

Burton, T.C. 1998a. Variation in the hand and superficial throat musculature of neotropical leptodactylid frogs. Herpetologica 54: 53-72.

Burton, T.C. 1998b. Pointing the way: the distribution and evolution of some characters of the finger muscles of frogs. American Museum Novitates 3229: $1-13$.

Burton, T.C. 1998c. Are the distal extensor muscles of the fingers of frogs adaptations to arboreality? Journal of Herpetology 32: 611-617.

Burton, T.C. 2001. Variation in the foot muscles of frogs of the family Myobatrachidae. Australian Journal of Zoology 49: 1-21.

Burton, T.C. 2004. Muscles of the pes of hylid frogs. Journal of Morphology 260: 209-233.

Cabra-García, J., and G. Hormiga. 2020. Exploring the impact of morphology, multiple sequence alignment and choice of optimality criteria in phylogenetic inference: a case study with the Neotropical orbweaving spider genus Wagneriana (Araneae: Araneidae). Zoological Journal of the Linnean Society 188: 976-1151.
Cannatella, D.C. 1985. A phylogeny of primitive frogs (Archeobatrachia). Ph.D. dissertation, Department of Systematics and Ecology, University of Kansas, Lawrence.

Cannatella, D.C., and L. Trueb. 1988. Evolution of pipoid frogs: morphology and phylogenetic relationships of Pseudhymenochirus. Journal of Herpetology 22: 439-456.

Chan, K.O., and R.M. Brown. 2017. Did true frogs 'dispersify'? Biological Letters 13: 20170299.

Chan, K.O., L.L. Grismer, and R.M. Brown. 2018. Comprehensive multi-locus phylogeny of Old World tree frogs (Anura: Rhacophoridae) reveals taxonomic uncertainties and potential cases of over-and underestimation of species diversity, Molecular Phylogenetics and Evolution 127: 1010-1019.

Channing, A. 2003. Ghost frogs (Heleophrynidae). In W.E. Duellman (editor), Grzimek's animal life encyclopedia, 2nd ed. Vol. 6. Amphibians: 131-134. Detroit: Gale Group.

Channing, A., M.-O. Rödel, and J. Channing. 2012. Tadpoles of Africa. The biology and identification of all known tadpoles in sub-Saharan Africa. Frankfurt am Main: Edition Chimaira.

Chen, J., G.S. Bever, H.Y, Yi, and M.A. Norell. 2016, A burrowing frog from the late Paleocene of Mongolia uncovers a deep history of spadefoot toads (Pelobatoidea) in East Asia. Scientific Reports 6: 19209.

Chen, J.-M., et al, 2017. A novel nuultilocus phylogenetic estimation reveals unrecognized diversity in Asian horned toads, genus Megophrys sensu lato (Anura: Megophryidae). Molecular Phylogenetics and Evolution 106: 28-43.

Coloma, L.A., et al. 2010. Five new (extinct?) species of Atelopus (Anura: Bufonidae) from Andean Colombia, Ecuador, and Peru. Zootaxa 2574: 1-54.

Darst, C.R., and D.C. Cannatella. 2004. Novel relationships among hyloid frogs inferred from $12 \mathrm{~S}$ and $16 \mathrm{~S}$ mitochondrial DNA sequences. Molecular Phylogenetics and Evolution 31: 462-475.

Davies, M., and T.C. Burton. 1982. Osteology and myology of the gastric brooding frog Rheobatrachus silus Liem (Anura: Leptodactylidae). Australian Journal of Zoology 30: 503-521.

de Sá, R.O., T. Grant, A. Camargo, W.R. Heyer, M.L. Ponssa, and E. Stanley. 2014. Systematics of the neotropical genus Leptodactylus Fitzinger, 1826 (Anura: Leptodactylidae): phylogeny, the relevance of nonmolecular evidence, and species accounts. South American Journal of Herpetology 9: SI-S128. 
Diogo, R, and V. Abdala. 2010. Muscles of vertebrates: comparative anatomy, evolution, homologies and development. Oxford: Taylor \& Francis.

Diogo, R., and J. Molnar. 2014. Comparative anatomy, evolution, and homologies of tetrapod hindlimb muscles, comparison with forelimb muscles, and deconstruction of the forelimb-hindlimb serial homology hypothesis. Anatomical Record 297: 1047-1075.

Diogo, R., and E.M. Tanaka. 2012. Anatomy of the pectoral and forelimb muscles of wildtype and green fluorescent protein-transgenic axolotls and comparison with other tetrapods including humans: a basis for regenerative, evolutionary and developmental studies. Journal of Anatomy 221: 622-635.

Diogo, R., and J.M. Ziermann. 2014. Development of fore- and hindlimb muscles in frogs: morphogenesis, homeotic transformations, digit reduction, and the forelimb-hindlimb enigma. Journal of Experimental Zoology Part B: Molecular and Developmental Evolution 322B: 86-105.

Diogo, R., S. Walsh, C. Smith, J.M. Ziermann, and V. Abdala. 2015. Towards the resolution of a longstanding evolutionary question: muscle identity and attachments are mainly related to topological position and not to primordium or homeotic identity of digits. Journal of Anatomy 226: 523-529.

Diogo, R., Z.M. Ziermann, J. Molnar, N. Siomava, and V. Abdala. 2018. Muscles of Chordates. Boca Raton: CRC Press.

Dos Santos, D.A., M.L. Ponssa, M.J. Tulli, and V. Abdala. 2014. Fibrillar organization in tendons: A pattern revealed by percolation characteristics of the respective geometric network. Network Biology 4: 31-46.

Dos Santos, D.A., J. Fratani, M.L. Ponssa, and V. Abdala. 2017. Network architecture associated with the highly specialized hindlimb of frogs. PloS ONE 12: $\mathrm{e} 0177819$

Drewes, R.C. 1984. A phylogenetic analysis of the Hyperoliidae (Anura): Treefrogs of Africa, Madagascar, and the Seychelles islands. Occasional Papers of the California Academy of Sciences 139: 1-70.

Duellman, W.E., and L. Trueb. 1986. Biology of amphibians. New York: McGraw-Hill.

Dugès, A. 1834. Recherches sur l'ostéologie et la myologie des Batraciens à leurs différens âges. Paris: J. B. Baillibre.

Dunlap, D.G. 1960. The comparative myology of the pelvic appendage in the Salientia. Journal of Morphology 106: 1-76.
Ecker, A. 1864. Die Anatomie des Frosches: Ein Handbuch für Physiologen, Ärzte und Studirende. Braunschweig: Vieweg \& Sohn.

Ecker, A. 1889. The anatomy of the frog. Oxford: Clarendon Press.

Egan, D. 1995. The comparative osteology and myology of the carpus of the Australian Leptodactylidae. Honours dissertation, Department of Zoology, University of Adelaide.

Fabrezi, M. 1992. El carpo de los anuros. Alytes 10: 1-29.

Fabrezi, M. 1993. The anuran tarsus. Alytes 11: 47-63.

Fabrezi, M., and P. Alberch. 1996. The carpal elements of anurans. Herpetologica 52: 188-204.

Fabrezi, M, and J.A, Langone. 2000, Los caracteres morfológicos del controvertido neobatrachia arborícola Allophryne ruthveni Gaige, 1926. Cuadernos de Herpetologia 14: 47-59.

Fabrezi, M., I. Goldberg, and M. Chuliver Pereyra. 2017. Morphological variation in anuran limbs: Constraints and novelties. Journal of Experimental Zoology Part B: Molecular and Developmental Evolution 328B: 546-574.

Faivovich, J. 2002. A cladistic analysis of Scinax (Anura: Hylidae). Cladistics $18 ; 367-393$.

Faivovich, J., et al, 2005. Systematic review of the frog family Hylidae, with special reference to Hylinae: phylogenetic analysis and taxonomic revision. Bulletin of the American Museum of Natural History 294: 1-240.

Faivovich, J., et al. 2018. On the monophyly and relationships of several genera of Hylini (Anura: Hylidae: Hylinae), with comments on recent taxonomic changes in hylids. South American Journal of Herpetology 13: 1-32.

Feng, Y.J., et al. 2017. Phylogenomics reveals rapid, simultaneous diversification of three major clades of Gondwanan frogs at the Cretaceous-Paleogene boundary. Proceedings of the National Academy of Sciences 114: E5864-E5870.

Fitch, W.M. 1971. Toward defining the course of evolution: minimum change for a specific tree topology. Systematic Zoology 20: 406-416.

Ford, L.S., and D.C. Cannatella. 1993. The major clades of frogs. Herpetological Monographs 7: 94-117.

Fouquet, A., et al. 2013. Unexpected phylogenetic positions of the genera Rupirana and Crossodactylodes reveal insights into the biogeography and reproductive evolution of leptodactylid frogs, Molecular Phylogenetics and Evolution 67: 445-457. 
Francis, E.T.B. 1934. The anatomy of the salamander. Oxford: Clarendon Press.

Fratani, J., M.L. Ponssa, and V. Abdala. 2018. Tendinous framework of anurans reveals an all-purpose morphology. Zoology 126: 172-184.

Fratani, J., M.L. Ponssa, M. Rada, and V. Abdala. 2020. The influence of locomotion and habitat use on tendo-muscular units of an anuran clade (Anura, Diphyabatrachia). Zoologischer Anzeiger 284: 66-77.

Frazão, A., H.R. da Silva, and C.A. de Moraes Russo. 2015. The Gondwana breakup and the history of the Atlantic and Indian oceans unveils two new clades for early neobatrachian diversification. PloS one 10: e0143926.

Frost, D.R. 2019. Amphibian Species of the World: an Online Reference. Version 6.0. American Museum of Natural History, New York. Internet resource (http://research.amnh.org/herpetology/amphibia/ index.html), accessed October 26, 2019.

Frost, D.R., et al. 2006. The amphibian tree of life. Bulletin of the American Museum of Natural History 297: 1-370.

Garcia-Paris, M., D.R. Buchholz, and G. Parra-Olea. 2003. Phylogenetic relationships of Pelobatoidea reexamined using mtDNA. Molecular Phylogenetics and Evolution 28: 12-23.

Garg, S., and S.D. Biju. 2019. New microhylid frog genus from Peninsular India with Southeast Asian affinity suggests multiple Cenozoic biotic exchanges between India and Eurasia. Scientific Reports 9: 1906.

Gaupp, E. 1895. Mitteilungen zur anatomie des frosches. II. Hand- und fubmuskeln des frosches. Anatomischer Anzeiger 11: 193-222.

Gaupp, E. 1896. A. Ecker's und Wiedersheim's Anatomie des Frosches. I. Braunschweig: Vieweg \& Sohn.

Gaupp, E. 1899. A. Ecker's und Wiedersheim's Anatomie des Frosches. II. Braunschweig: Vieweg \& Sohn.

Goloboff, P.A., and S.A. Catalano. 2016. TNT version 1.5 , including a full implementation of phylogenetic morphometrics. Cladistics 32: 221-238.

Grant, T., and A.G. Kluge. 2004. Transformation series as an ideographic character concept. Cladistics 20: 23-31.

Grant, T., and A.G. Kluge. 2009. Parsimony, explanatory power, and dynamic homology testing. Systematics and Biodiversity 7: 357-363.

Grant, T., et al. 2006. Phylogenetic systematics of dartpoison frogs and their relatives (Amphibia: Athesphatanura: Dendrobatidae). Bulletin of the American Museum of Natural History 299: 1-262.
Grant, T., et al. 2017. Phylogenetic systematics of dartpoison frogs and their relatives revisited (Anura: Dendrobatoidea). South American Journal of Herpetology 12: S1-S90.

Haas, A. 1995. Cranial features of dendrobatid tadpoles (Amphibia: Anura: Dendrobatidae). Journal of Morphology 224: 241-264.

Haas, A. 2003. Phylogeny of frogs as inferred from primarily larval characters (Amphibia: Anura). Cladistics 19: 23-89.

Haines, R.W. 1939. A revision of the extensor muscles of the forearm in tetrapods. Journal of Anatomy 73: 211-233.

Hanna, G., and W.J.P. Barnes. 1991. Adhesion and detachment of the toe pads of tree frogs. Journal of Experimental Biology 155: 103-125.

Hayashi, S., et al. 2015. Evidence for an amphibian sixth digit. Zoological Letters 1: 17.

Heinicke, M.P., et al. 2009. A new frog family (Anura: Terrarana) from South America and an expanded direct-developing clade revealed by molecular phylogeny. Zootaxa 2211: 1-35.

Hennig, W. 1966. Phylogenetic systematics. Chicago: University of Illinois Press.

Hoffmann, C.K. 1878. Bronn's Klassen und Ordnungen des Thier-reichs. VI. Leipzig und Heidelberg.

Holmes, R. 1977. The osteology and musculature of the pectoral limb of small captorhinids. Journal of Morphology 152: 101-140.

Hoyos, J.M., and L. Salgar. 2016. New conditions and intraspecific variation of some muscles of hands and feet of Dendropsophus labialis (Peters, 1863) (Anura, Hylidae). Acta Zoologica 97: 143-153.

Hoyos, J.M., C. Mantilla, D. Galindo, and L. Salgar. 2014. Phylogenetic analysis whithin the Pristimantis unistrigatus (Anura, Craugastoridae) group based on morphological characters. Caldasia 36: 107-124.

Humphry, G.M. 1871. The muscles and nerves of the Cryptobranchus japonicus. Journal of Anatomy and Physiology 6: 1-61.

Hurley, I., M.E. Hale, and V.E. Prince. 2005. Duplication events and the evolution of segmental identity. Evolution \& Development 7: 556-567.

Inger, R.F. 1972. Bufo of Eurasia. In Blair, W.F. (editor), Evolution in the genus Bufo. Austin: University of Texas Press.

Janani, S.J., K. Vasudevan, E. Prendini, S.K. Dutta, and R.K. Aggarwal. 2017. A new species of the genus Nasikabatrachus (Anura, Nasikabatrachidae) from the eastern slopes of the Western Ghats, India. Alytes 34: 1-19. 
Jetz, W., and R.A. Pyron. 2018. The interplay of past diversification and evolutionary isolation with present imperilment across the amphibian tree of life. Nature Ecology \& Evolution 2: 850.

Kändler, R. 1924. Die sexuelle Ausgestaltung der Vorderextremität der anuren Amphibien. Jenaische Zeitschrift für Naturwissenschaft 60: 175-240.

Kluge, A.G. 1997. Testability and the refutation and corroboration of cladistic hypotheses. Cladistics 13: $135-175$.

Kurabayashi, A., and M. Sumida. 2013. Afrobatrachian mitochondrial genomes: genome reorganization, gene rearrangement mechanisms, and evolutionary trends of duplicated and rearranged genes. BMC Genomics 14: 1-17.

Langowski, J.K.A., et al. 2018. Force-transmitting structures in the digital pads of the tree frog Hyla cinerea: a functional interpretation. Journal of Anatomy 233: 478-495.

Laurent, R.F. 1986. Sous classe de Lissamphibiens: Lissamphibia Systématique. In P.-P. Grassé, and M. Delsol (editors), Traité de Zoologie: Anatomie, Systématique, Biologie, Tome XIV: 594-797. Paris: Masson.

Liem, D.S.S. 1970. The morphology, systematics, and evolution of the Old World treefrogs (Rhacophoridae and Hyperoliidae). Fieldiana Zoology 57: 1-145.

Liem, D.S.S. 1973. A new genus of frog of the family Leptodactylidae from SE. Queensland, Australia. Memoirs of the Queensland Museum 16: 459-470.

Liem, D.S.S., and W. Hosmer, 1973. Frogs of the genus Taudactylus with description of two new species (Anura: Leptodactylidae). Memoirs of the Queensland Museum 16: 435-457.

Lynch, J.D. 1973. The transition from archaic to advanced frogs. In J.L. Vial (editor), Evolutionary biology of the anurans. Contemporary research on major problems: 133-182. Columbia; University of Missouri Press.

Maddison, W., and D. Maddison. 2015. Mesquite: a modular system for evolutionary analysis. Version 3.03 (7 May 2015). Online resource (http://mesquiteproject.org).

Maglia, A.M. 1998. Phylogenetic relationships of extant pelobatoid frogs (Anura: Pelobatoidea): evidence from adult morphology. Scientific Papers. Natural History Museum, University of Kansas 10: 1-19.

Maglia, A.M. 2003. Skeletal development of Pelobates cultripes and a comparison of the osteogenesis of pelobatid frogs (Anura: Pelobatidae). Scientific Papers. Natural History Museum, University of Kansas 30: 1-13.
Mahony, S., N.M. Foley, S.D. Biju, and E.C. Teeling. 2017. Evolutionary history of the Asian Horned Frogs (Megophryinae): integrative approaches to timetree dating in the absence of a fossil record. Molecular Biology and Evolution 34: 744-771.

Manzano, A.S. 1996. Análisis de la musculatura de la familia Pseudidae (Amphibia: Anura). Ph.D. dissertation, Universidad Nacional de Tucumán, Argentina.

Manzano, A.S., M. Fabrezi, and M. Vences. 2007. Intercalary elements, treefrogs, and the early differentiation of a complex system in the Neobatrachia. Anatomical Record 290: 1551-1567.

Manzano, A.S., V. Abdala, and A. Herrel. 2008. Morphology and function of the forelimb in arboreal frogs: specializations for grasping ability? Journal of Anatomy 213: 296-307.

McMurrich, J.P. 1903a. The phylogeny of the forearm flexors. American Journal of Anatomy 2: 177-209.

McMurrich, J.P. 1903b. The phylogeny of the palmar musculature. American Journal of Anatomy 2: 463500 .

Mirande, J.M. 2017. Combined phylogeny of ray-finned fishes (Actinopterygii) and the use of morphological characters in large-scale analyses. Cladistics 33: $333-350$.

Moczek, A.P. 2008. On the origins of novelty in development and evolution. BioEssays 30: 432-447.

Müller, G.B. 2010. Epigenetic innovation. In M. Pigliucci, and G.B. Müller (editors), Evolution-the extended synthesis: 307-332. Cambridge: MIT Press.

Muzzopappa P., L.A. Púgener, and A.M. Báez. 2016. Postcranial osteogenesis of the helmeted water toad Calyptocephalella gayi (Neobatrachia: Calyptocephalellidae) with comments on the osteology of australobatrachians. Journal of Morphology 277: 204-230.

Nussbaum, R.A. 1982. Heterotrophic bones in the hindlimbs of frogs of the families Pipidae, Ranidae and Sooglossidae. Herpetologica 38: 312-320.

Nussbaum, R.A., and S.-H. Wu. 2007. Morphological assessments and phylogenetic relationships of the seychellean frogs of the family Sooglossidae (Amphibia: Anura). Zoological Studies 46: 322-335.

Nylander, J.A., F. Ronquist, J.P. Huelsenbeck, and J. Nieves-Aldrey. 2004. Bayesian phylogenetic analysis of combined data. Systematic Biology 53; 47-67.

Oka, Y., R. Ohtani, M. Satou, and K. Ueda. 1984. Sexually dimorphic muscles in the forelimb of the Japanese toad, Bufo japonicus. Journal of Morphology 180: $297-308$. 
O'Leary, M.A., et al. 2013. The placental mammal ancestor and the post-K-Pg radiation of placentals. Science 339: 662-667.

Oliveira-Lagôa, S.D., F.B. Cruz, A.D.L. Moreno, E.O. Lavilla, and V. Abdala. 2019. Anuran forelimb muscle tendinous structures and their relationship with locomotor modes and habitat use. Current Zoology 65: 599-608.

Pabijan, M., et al. 2013. Complete mitochondrial genomes resolve phylogenetic relationships within Bombina (Anura: Bombinatoridae). Molecular Phylogenetics and Evolution 69: 63-74.

Padial, J.M., T. Grant, and D.R. Frost. 2014. Molecular systematics of terraranas (Anura: Brachycephaloidea) with an assessment of the effects of alignment and optimality criteria. Zootaxa 3825: 1-132.

Patterson, C. 1982. Morphological characters and homology. In K.A. Joysey, and E. Friday (editors), Problems of Phylogenetic Reconstruction: 21-74. New York: Academic Press.

Pereyra, M.O., et al. 2016. The complex evolutionary history of the tympanic middle ear in frogs and toads (Anura). Scientific Reports 6: 34130.

Perrin, A. 1891. Sur les muscles du pied de la Rana. Bulletin Scientifique de la France et de la Belgique 3: $16-20$.

Perrin, A. 1892. Contribution a l'étude de la myologie comparée: Membre postérieur chez certain nombre de Batrachiens et de Sauriens. Bulletin Scientifique de la France et de la Belgique 24: 373-552.

Perrin, A. 1899. Contribution a l'étude de la myologie et de I'ostéologie comparée. Membre antérieur chez un certain nombre de Batraciens et de Sauriens. Bulletin Scientifique de la France et de la Belgique 32: 220-282.

Peterson, T., and G.B. Müller. 2013. What is evolutionary novelty? Process versus character based definitions: Journal of Experimental Zoology Part B: Molecular and Developmental Evolution 320: 345-350.

Pigliucci, M. 2008. What, if anything, is an evolutionary novelty? Philosophy of Science 75: 887-898.

Pinna, M.C. de. 1991. Concepts and tests of homology in the cladistic paradigm. Cladistics 7: 367-394.

Púgener, L.A., A.M. Maglia, and L. Trueb. 2003. Revisiting the contribution of larval characters to an analysis of phylogenetic relationships of basal anurans. Zoological Journal of the Linnean Society 139: 129-155.

Pyron, R.A. 2014. Biogeographic analysis reveals ancient continental vicariance and recent oceanic dispersal in amphibians. Systematic Biology 63: 779-797.

Pyron, R.A., and J.J. Wiens. 2011. A large-scale phylogeny of Amphibia including over 2,800 species, and a revised classification of extant frogs, salamanders, and caecilians. Molecular Phylogenetics and Evolution 61: 543-58

Radhakrishnan, C., K.C. Gopi, and M.J. Palot. 2007. Extension of range of distribution of Nasikabatrachus sahyadrensis Biju \& Bossuyt (Amphibia: Anura: Nasikabatrachidae) along Western Ghats, with some insights into its bionomics. Current Science 92: 213-216.

Raj, P., et al. 2012. Larval morphology and ontogeny of Nasikabatrachus sahyadrensis Biju \& Bossuyt, 2003 (Anura, Nasikabatrachidae) from Western Ghats, India. Zootaxa 3510: 65-76.

Ramírez, M.J. 2007. Homology as a parsimony problem: a dynamic homology approach for morphological data. Cladistics 23: 588-612.

Restrepo, A.E., and J.M. Hoyos. 1998. Musculatura de los miembros y de las cinturas en Bolitoglossa adspersa (Peters, 1863) (Urodela, Plethodontidae). Alytes 16: 25-49.

Ribbing, L. 1907. Die distale Armmuskulatur der Amphibien, Reptilien und Säugetiere. Zoologishe Jahrbucher 23: 587-680.

Ribbing, L. 1909. Die Unterschenkel- und Fussmuskulatur der Tetrapoden und ihr Verhalten $\mathrm{zu}$ der entsprechenden Arm- und Handmuskulatur. Lunds Universitetes Årsskrift 5: 1-158.

Ribbing, L. 1911. Die distale Extremitätenmuskulatur von Pipa. Lunds Universitetes Årsskrift 6: 10-25.

Robovská-Havelková, P. 2010. How can ontogeny help us to understand the morphology of anuran pectoral girdle? Zoomorphology 129: 121-132.

Roelants, K., and F. Bossuyt. 2005. Archaeobatrachian paraphyly and pangaean diversification of crowngroup frogs. Systematic Biology 54: 111-126.

Roelants, K., et al. 2007. Global patterns of diversification in the history of modern amphibians. Proceedings of the National Academy of Sciences 104: 887-92.

Sabaj, M.H. 2016. Standard symbolic codes for institutional resource collections in herpetology and ichthyology: an Online Reference. Version 6: 802-832.

Salgar, L., J.M. Hoyos, and A. Acosta. 2009. New conditions and intraspecific variations in hand and foot muscles in Pristimantis bogotensis (Peters, 1863) (Amphibia: Anura: Strabomantidae). Zootaxa 2025: 56-68. 
Sánchez-Pacheco, S.J., et al. 2018. Phylogeny of Riama (Squamata: Gymnophthalmidae), impact of phenotypic evidence on molecular datasets, and the origin of the Sierra Nevada de Santa Marta endemic fauna. Cladistics 34: 260-291.

San Mauro, D. 2010. A multilocus timescale for the origin of extant amphibians. Molecular Phylogenetics and Evolution 56: 554-561.

San Mauro, D., M. Vences, M. Alcobendas, R. Zardoya, and A. Meyer. 2005. Initial diversification of living amphibians predated the breakup of Pangaea. The American Naturalist 165: 590-599.

Scott, E. 2005. A phylogeny of ranid frogs (Anura: Ranoidea: Ranidae), based on a simultaneous analysis of morphological and molecular data. Cladistics 21: $507-574$.

Senevirathne, G., et al. 2016. From clinging to digging: The postembryonic skeletal ontogeny of the Indian Purple Frog, Nasikabatrachus sahyadrensis (Anura: Nasikabatrachidae). PLoS ONE 11; e0151114.

Silva, H.R. 1998. Phylogenetic relationships of the family Hylidae with emphasis on the relationships within the subfamily Hylinae (Amphibia: Anura). Ph.D. dissertation, Department of Systematics and Ecology, University of Kansas.

Sokol, O,M. 1975. The phylogeny of anuran larvae: a new look. Copeia 1975: 1-23.

Sokol, O.M. 1977. A subordinal classification of frogs. Journal of Zoology 182: 505-508.

Soliz, M., M.J. Tulli, and V. Abdala. 2020. Forelimb musculoskeletal-tendinous growth in frogs. Peer) 8: e8618.

Streicher, J.W., et al. 2018. Evaluating methods for phylogenomic analyses, and a new phylogeny for a major frog clade (Hyloidea) based on 2214 loci. Molecular Phylogenetics and Evolution 119: 128143.

Trueb, L. 1993. Patterns of cranial diversity among the Lissamphibia. In J. Hanken, and B.K. Hall (editors), Patterns of Structural and Systematic Diversity: 255-343. Chicago: University of Chicago Press.

Trueb, L., C.F. Ross, and R. Smith. 2005. A new pipoid anuran from the Late Cretaceous of South Africa. Journal of Vertebrate Paleontology 25: 533-547.

Tu, N., M. Yang, D. Liang, and P. Zhang. 2018. A largescale phylogeny of Microhylidae inferred from a combined dataset of 121 genes and 427 taxa. Molecular Phylogenetics and Evolution 126: 85-91.

Tyson, H.L. 1987. The structure and development of the anuran breast-shoulder apparatus, forelimb, and associated musculature. Ph.D. dissertation, University of Alberta, Canada.

Wake, D.B., M.H. Wake, and C.D. Specht. 2011. Homoplasy: From detecting pattern to determining process and mechanism of evolution. Science 331 : 1032-1035.

Walthall, J.C., and M.A. Ashley-Ross. 2006. Postcranial myology of the California newt, Taricha torosa. The Anatomical Record Part A 288A; 46-57.

Wiens, J.J., J.W. Fetzner Jr, C.L. Parkinson, and T.W. Reeder. 2005. Hylid frog phylogeny and sampling strategies for speciose clades. Systematic Biology 54: 719-748.

Wiens, J.J., C.A. Kuczynski, X. Hua, and D.S. Moen. 2010. An expanded phylogeny of treefrogs (Hylidae) based on nuclear and mitochondrial sequence data. Molecular Phylogenetics and Evolution 55: 871882.

Wilkinson, E. 1993. Evolution of burrowing in three lineages of Australian frogs. B.Sc. dissertation, University of Adelaide.

Young, R.L., G.S. Bever, Z. Wang, and G.P. Wagner. 2011. Identity of the avian wing digits: problems resolved and unsolved. Developmental Dynamics 240: 1042-1053.

Zachariah, A., R.K. Abraham, S. Das, K.C. Jayan, and R. Altig. 2012. A detailed account of the reproductive strategy and developmental stages of Nasikabatrachus sahyadrensis (Anura: Nasikabatrachidae), the only extant member of an archaic frog lineage. Zootaxa 3510: 53-64.

Zhang, P., et al. 2013. Efficient sequencing of anuran mtDNAs and a mitogenomic exploration of the phylogeny and evolution of frogs. Molecular Biology and Evolution 30: 1899-1915. 


\section{APPENDIX 1}

\section{SPECIMENS Examined}

\section{Collection Codes}

Collection abbreviations are as follow: $\mathrm{BB}$ (Boris Blotto field series; to be accessioned in MACN); CAS (California Academy of Sciences); CENAI (Centro Nacional de Investigaciones Iológicas, Buenos Aires, Argentina; housed in MACN); CFBH (Collection Célio F,B. Haddad, Universidade Estadual Paulista, Rio Claro, São Paulo, Brazil); CM (Carnegie Museum, Pittsburgh, Pennsylvania); FMNH (Field Museum, Chicago); ICN (Universidad Nacional de Colombia, Instituto de Ciencias Naturales, Museo de Historia Natural, Bogotá, Colombia); KU (University of Kansas Natural History Museum, Lawrence, Kansas); LGE (Laboratorio de Genética Evolutiva, Universidad Nacional de Misiones); MACN (Museo Argentino de Ciencias Naturales "Bernardino Rivadavia," Buenos Aires, Argentina); MNRJ (Universidade Federal de Rio de Janeiro, Museu Nacional do Rio de Janeiro, Rio de Janeiro, Brazil); MZUSP (Museu de Zoologia, Universidade de São Paulo, São Paulo, Brazil); SB (Sebastián Barrionuevo field series; to be accessioned in Fundación Miguel Lillo); TG (Taran Grant field series); UFMT (Universidade Federal de Mato Grosso); UMMZ (University of Michigan, Museum of Zoology, Michigan); ZUEC (Museu de História Natural, Universidade Estadual de Campinas, Campinas, Brazil).

\section{Specimens and Locality Data}

Acris crepitans: MACN 39139: United States: New Jersey: Cape May: Cape May, 1 mi S Erma (Lake Laurie Camp), Adelotus brevis: KU 179907: Australia: Queensland: Jimna St Forest, Central Burnett hwy at jet with Marumbah 4 road. Adelphobates galactonotus: MZUSP 147784: Brazil: Para: Porto de Moz. Afrixalus fulvovittatus: KU 291836: Guinea: vicinity of stream by hotel Sala, ca. $2.5 \mathrm{~km}$ S Labe, Allophryne ruthveni: MZUSP 78207: Brazil: Rondonia: UHE Samuel; Brazil:
Mato Grosso: Apiacás. Alsodes neuquensis: MACN 41370: Argentina: Neuquén: Aluminé: Volcán Batea Mahuida; MACN 48996: Argentina: Neuquén: Aluminé: Pampa de Lonco Luan on Provincial Route 13; MACN 49005: Argentina: Neuquén: Aluminé: stream around $10 \mathrm{~km}$ W Primeros Pinos. Alytes obstetricans: CM 60824: Spain: Asturias: Asturias: Oviedo, Lago de la Ercina, east side, MZUSP 11472; Belgium. Amazophrynella minuta: ICN 46770: Colombia: Amazonas: Leticia: Km. 13.8 Leticia - Tarapaca. Anaxyrus woodhousii: MACN 42131, MACN 42132: No data (pet trade). Aplastodiscus perviridis: CFBH 11206: Brazil: Minas Gerais: Camanducaia: Monte Verde; MACN 35170: Argentina: Misiones: Guaraní: INTA cuartel Río Victoria. Arthroleptis variabilis: KU 204646: Gabon: Ogooue-Ivindo: M'Passa Field Station (IRET), ca. 10 km WSW Makokou. Ascaphus truei: CENAI s/ $\mathrm{n}^{\circ}$ : No datá; KU 153228: United States: Washington: Pierce: Narada Falls. Atelognathus patagonicus; MACN 37881, MACN 37883: Argentina: Neuquén: Catán Lil: Laguna del Burro. Atelopus pastuso: ICN 55481: Colombia, Nariño. Barbourula busuangensis: KU 324606: Philippines: Palawan: Narra: Barangay Estrella, Estrella Falls Park. Barycholos ternetzi: CFBH 13451: Brazil: São Paulo: Pedregulho: Parque Estadual das Furnas do Bom Jesus. Batrachyla leptopus: MACN 48995, MACN 52416: Argentina: Chubut: near Desemboque; MACN 42534: Argentina: Rio Negro: Bariloche: 3 to $4 \mathrm{~km}$ from Pampa Linda in direction to Cerro Tronador. Boana cordobae: MACN 37696: Argentina: San Luis: Chacabuco: Cortaderas, Villa Elena, La Calera stream. MACN 48999: Argentina: Córdoba: near Pampilla. Bombina bombina: KU 68137: Austria: Wien-Stadlau: Innundationsgebiet der Donau. Bombina orientalis: KU 38654: Korea: 2 mi N Yongdae-ri. MACN 42130: No data (pet trade). Bombina variegata: FMNH 62475: Germany. Breviceps adspersus: CAS 170339: Swaziland: Lubombo Dist.: top of Lebombo Mtns., above Big Bend; CENAI 5832: South Africa: KwaZuluNatal: Durban. Cacosternum capense: CENAI 6426: South Africa: Cape Town: Durbanville. 
Calyptocephalella gayi: CENAI 636, CENAI s/n: No data. Cornufer guentheri: KU 307395: Solomon Islands: Guadalcanal Province: Guadalcanal Island: Tadai District, Mt. Austen, Village of Barana (on tributary of Lunga River). Ceratophrys cranwelli: MACN 42339, MACN 49007: Argentina: Santa Fe: Vera: Estancia "Las Gamas." Chacophrys pierottii: CENAI 10940: Argentina: Córdoba: Totoralejos. MACN 48997: Argentina: Salta: km 1786 RN81, near Dragones. Chaltenobatrachus grandisonae: BB 2341: Argentina: Santa Cruz: Salto del Anillo, near Lago del Desierto. Chaperina fusca: KU 319440: Philippines: Agusan del Sur Province: Municipality of San Francisco: Barangay Bagusan II, Mt. Magdiwata. Chiromantis xerampelina: KU 195919: South Africa: Natal: $3 \mathrm{~km}$ W Mtubatuba. Craugastor fitzingeri: MACN 39163: Panamá: La Chorrera: Barro Colorado. Crinia signifera: KU 56641: Australia: New South Wales: $55 \mathrm{mi} \mathrm{NE}$ Armidale, Point Lookout. Crossodactylus schmidti: MACN 35124, MACN 48998: Argentina: Misiones: Guaraní: INTA cuartel Río Victoria. Ctenophryne

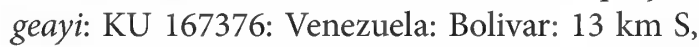
$1 \mathrm{~km}$ E Puente Cuyuni. Cycloramphus boraceiensis: CFBH 17297: Brazil: RJ: Paraty: Estrada Paraty-Cunha. CFBH 17308: Brazil: RJ: Paraty: Estrada Paraty-Cunha. Dendrophryniscus leucomystax: CFBH 22328: Brazil: SP: Cananéia: Ilha de Cananéia. Dendropsophus luddeckei: ICN 12586: Colombia: Boyacá: Duitama: Paramo La Rusia, Km. 19-21 de la carretera DuitamaCharala. Dendropsophus minutus: MACN 41973: Argentina: Misiones: San Pedro: Access to Parque Provincial Cruce Caballero. MACN 43286, MACN 45095, MACN 45097: Argentina: Misiones: Guaraní: San Vicente, INTA, Cuartel Río Victoria. Discoglossus galganoi: CM 52531: Spain: Andalucía: Cádiz: 3.3-7.2 mi SW Arcos de la Frontera, C-343. Discoglossus pictus: MZUSP 94898: Tunisia: Bizerta. Elachistocleis bicolor: BB 1790: Argentina: Santa Fe: near Alto Verde. Eleutherodactylus coqui: KU 180490: Puerto Rico: Humacao: Caribbean National Forest, Big Tree Trail, El Yunque. Espadarana prosoblepon: ICN 19657: Colombia: Antioquia:
Urrao: Parque Natural Nacional Las Orquideas, Vereda Calles, Quebrada Honda. Eupsophus roseus: MACN 52414: Argentina: Neuquén: Lago Currhue Grande, margin S; MACN 49594: Argentina: Neuquén: Huiliches: Trail to Lago Paimún, próx. Paso Carirriñe. Fejervarya vittigera: KU 326281: Philippines: Antique Province: Municipality of San Jose. Flectonotus pygmaeus: KU 133363: Venezuela: Aragua: Estación Biológica Rancho Grande. Gastrotheca christiani: CENAI 3204: Argentina: Jujuy: Ledesma - Valle Grande. Geocrinia victoriana: KU 186877: Australia: Victoria: 96 km NW Melbourne, Mt Erica. Glyphoglossus guttulatus: KU 328346: Thailand: Nakhon Ratchisma Province: Nakhon Ratchisma District: Sakaerat Environmental Research Station (SERS). Haddadus binotatus: MACN 17042, MACN 17048: Brazil: Alto da Serra Paranapiacaba. Hadromophryne natalensis: FMNH 82388: South Africa: Natal. Heleophryne orientalis: FMNH 187311: South Africa: Cape. Heleophryne purcelli: FMNH 250016: South Africa. Hemiphractus proboscideus: KU 123141: Ecuador: Sucumbios: Santa Cecilia. Hemisus marmoratus: MACN 39130: Tanzania: Mara: Serengeti: Tarangire National Park. Hoplobatrachus rugulosus: KU 305458: Philippines: Occidental Mindoro Province: Municipality of Calintaan: Barangay Malpalon. Hylodes phyllodes: MACN 17252: Brazil: São Paulo: Boracéia. Hylorina sylvatica: MACN 42530: Argentina: Neuquén: Laguna Pire. Hymenochirus boettgeri: FMNH 109254: Democratic Republic of the Congo: Kivu. Hymenochirus curtipes: KU 204143: Democratic Republic of the Congo: Kinshasa. Hyperolius semidiscus: KU 195874: South Africa: Natal: Ashburton. Insuetophrynus acarpicus: CENAI 6898: Chile: IX Región: Valdivia: Mehuín. Ischnocnema guentheri: MACN 17058: Brazil: Alto da Serra Paranapiacaba. Kalophrynus sinensis: KU 309647: Philippines: Camiguin: Mambajao: Barangay Pandan, sitio Kampana. Kaloula pulchra: MACN 39169: No data (pet trade). Kassina senegalensis: CENAI 5932: South Africa: Natal: Durban. Lechriodus melanopyga: KU 133626: Papua New Guinea: Central: Brown River, 25 mi NW Port 
Moresby. Leiopelma hochstetteri: UMMZ 177464: New Zealand. Lepidobatrachus laevis: MACN 52418: Argentina: RN 81 entre Dragones (provincia de Salta) e Ingeniero Juarez (provincia de Formosa). Leptobrachium lumadorum: KU 334644: Philippines: Pasonanca Natural Park, Sitio Lawin, Labuya Outpost. Leptodactylus latinasus: MACN 40726, MACN 49001: Argentina: Formosa: Laguna Yema. Leptobrachella bourreti: KU 331621: Vietnam: Dien Bien: Sin Thau Village, Muong Nhe Nature Reserve, Camp 1. Leptopelis natalensis: CENAI 5983: South Africa: Natal: Durban. Limnodynastes peronii: CENAI 8537: Australia: New South Wales: Normanhust. KU 179939: Australia: Queensland: Jimna State Forest, Central Burnett Hwy at jct Marumbah 4 Rd. Limnomedusa macroglossa: MACN 49006, MACN 52424: Argentina: Misiones: Aristóbulo del Valle: Cuña Pirú. Limnonectes woodworthi: KU 306143: Philippines: Quezon Province: Tayabas City: Barangay Kamaisa. Lithobates warszewitschii: KU 36318: Costa Rica: San Jose: La Palma. Macrogenioglottus alipioi: CFBH 12722, CFBH 13118: Brazil: São Paulo: Ubatuba: Picinguaba. CFBH 24476, CFBH 24477: Brazil: Espírito Santo: Santa Teresa: Reserva Biológica Augusto Ruschi. CFBH 36087: Brazil: Bahia: Uruçuca: Fazenda Bom Fim. MZUSP 126264: Brazil: Bahia: Una. Mannophryne herminae: CENAI 2616, CENAI 2621: Venezuela: Reserva Rancho Grande. Megophrys aceras: KU 327892: Thailand: Nakhon Si Thammarat: Khao Luang National Park, Krung Ching Waterfall. Megophrys carinense: FMNH 175473: Thailand: Chiang Mai. Megophrys edwardinae: FMNH 250931: Malaysia: Sabah: Ranau Dist. Melanophryniscus klappenbachi: LGE 16644, MACN 49593: Argentina: Chaco: San Fernando: Club Sixty Resistencia. Microbatrachella capensis: KU 207864: South Africa: Cape: $3 \mathrm{~km}$ E Kleinmond. Microhyla heymonsi: KU 311926: China: Guizhou: Libo County: Shuipu Village. Micryletta inornata: KU 328192: Thailand: Nakhon Ratchisma Province: Nakhon Ratchisma District: Sakaerat Environmental Research Station. Mixophyes iteratus: KU 179963: Australia: Queensland: Bellthorpe State
Forest, Stony Creek at Sunday Creek-Bellthorpe Rd. Nannophryne variegata: MACN 41475, MACN 52417: Argentina: Santa Cruz: Lago Argentino: Lago del Desierto. Neobatrachus aquilonius: KU 93576: Australia: Northern Territory Tennant Creek. Notaden bennettii: KU 175516: Australia: New South Wales Coonamble. Occidozyga laevis: KU 320181: Philippines: Occidental Mindoro Province: Municipality of Lubang: Barangay Vigo, Sitio Dangay. Odontophrynus achalensis: CENAI 2973: Argentina: Córdoba: Pampa de Achala; MACN 49009: Argentina: Córdoba: Cruz Del Eje: Los Gigantes. Odorrana versabilis: KU 311679: China: Guangxi: Shiwan Dashang Nature Reserve (Near town of Fulong). Oreobates barituensis: MACN 52411: Argentina: Salta: Quebrada El Lapachar, near Río Sidras, Parque Nacional Baritú. Oreolalax omeimontis: FMNH 272567: China: Sichuan: Hongya Xian. Osteopilus septentrionalis: KU 222212: Puerto Rico: Trujillo: Alto Cooperativa de Vivienda, Av. Periferal, Ciudad Univ. Paratelmatobius poecilogaster: CFBH 9879: Brazil: São Paulo: São Luis do Paraitinga: Núcleo Santa Virgínia - PESM. Pelobates cultripes: CM 53273: Spain: Andalucía: Cádiz: Puerto Real. Pelobates fuscus: CENAI 7548: No data. Pelobates varaldii: FMNH 109663: Morocco. Pelodytes ibericus: CM 52151: Spain: Andalucía: Cádiz: On C-440 [A-381], 3.9 - 13.7 Km SE of intersection with C-343 at Medina Sidonia. Philoria frosti: KU 186907: Australia: Victoria: Mt Baw Baw, 108.8 km NE Melbourne. Phrynobatrachus kreffii: CENAI 7623: Tanzania: Amani, Mt Usambara. Phrynomantis bifasciatus: MACN 42120: No data (pet trade). Phyllodytes luteolus: CFBH 890, CFBH 891: Brazil: Espírito Santo: Guaraparí: Restinga de Setiba; MNRJ 23274: Brazil: Bahia: Nova Viçosa. Phyllomedusa sauvagii: MACN 38136: Argentina: Formosa: Ramón Lista: María Cristina. MACN 42277: Argentina: Salta: National Route 81 between Dragones and Pluma de Pato. Physalaemus biligonigerus: MACN 52412: Argentina: Salta: km 1780 RN81, near Dragones. MACN 52413: Argentina: Salta: National Route 81 between km 1745 (Morillo) 
and km 1780. Pipa arrabali: KU 167442: Venezuela: Bolivar: Km 112, El Dorado-Santa Elena de Uairen rd. Pipa carvalhoi: MZUSP 81992, MZUSP 82005: Brazil: Bahia: Itapetinga. Pipa parva: KU 303252: Venezuela: Zulia: Santa Cruz del Zulia. Pipa pipa: MZUSP 142753: Brazil: Amazonas: São Sebastião: Margem esquerda do Rio Abacaxis. Platymantis guentheri: KU 333500: Philippines: Agusan del Norte Province: Municipality of Remedios T. Romualdez: Eye Falls, Intersection of Dayhopan and Agan Rivers, Mt. Hilong-hilong, $470 \mathrm{~m}$. Platyplectrum ornatum: CENAI 8544: Australia: Queensland: Coen Cape York. Pleurodema kriegi: MACN 38740: Argentina: Córdoba: Punilla: near Parador El Cóndor. Polypedates leucomystax: MACN 39174: No data (pet trade). Pristimantis buckleyi: KU 189961: Ecuador: Sucumbios: Santa Barbara. Proceratophrys boiei: CFBH 23193: Brazil: Paraná: Antonina: Fazenda Gervásio, Reserva Natural Rio Cachoeira. MZUSP 143563: Brazil: São Paulo: Parque Estadual de Carlos Botelho. Pseudhymenochirus merlini: KU 207823: Sierra Leone: Southern: Kasewe Forest Reserve. Pseudis minuta: CFBH 30003: Brazil: Rio Grande do Sul: Jaguarão; MACN 39253: Argentina: Entre Ríos: Islas del Ibicuy: Old National Route 12, at its intersection with the railway; MACN 40417, MACN 40418: Argentina: Entre Ríos: Islas del Ibicuy: Old National Route 12, next to its intersection with the railway; MACN 52409: Argentina: Entre Ríos: Islas del Ibicuy: Antigua Ruta 12 and Luciano stream. Pseudopaludicola falcipes: MACN 37875, MACN 37880: Argentina: Entre Ríos: Islas del Ibicuy: Old National Route 12, next to its intersection with Luciano stream. MACN 44616: Argentina: Buenos Aires: Campana: Bajos del Cazador, near Escobar. Pseudophryne coriacea: KU 179979: Australia: Queensland: Sunday Creek State Forest, W branch Kilcoy Crk at Bellthorpe-Jimna Rd. Ptychadena nilotica: MACN 39150: Tanzania: Mara: Serengeti: Tarangire National Park, Headquarters Pond No 2. Ranoidea eucnemis: MZUSP 36185: Papua New Guinea: Karimui, S. Highlands. Rhacophorus pardalis: KU 309826: Philip- pines: Camiguin Province: Municipality of Mambajao: Barangay Pandan, sitio Pamahawan. Rheobatrachus silus: KU 179995: Australia: Queensland: Sunday Creek State Forest, Kilcoy Creek. Rhinella fernandezae: MACN 39345: Argentina: Entre Ríos: Islas del Ibicuy: Old National Route 12, at its intersection with the railway. Rhinoderma darwinii: CENAI 5370: Chile: VIII Región: Concepción; CENAI 5469, CENAI 5491: Chile: IX Región: Valdivia: Mehuín. Rhinophrynus dorsalis: KU171202: Mexico: Campeche: $5 \mathrm{~km}$ N Escarcega; MACN 39131: Mexico: Tamaulipas: Altamira: Tamaulipas, 2,6 mi NW of Altamira. Sanguirana sanguinea: KU 309020: Philippines: Palawan Province: Municipality of Puerto Princessa: Barangay Irawan: Irawan Watershed. Scaphiopus couchii: KU 47023: Mexico: Coahuila: 2-6 mi W Sacramento. KU 200878: Mexico: Nuevo Leon: $15.4 \mathrm{~km} \mathrm{~N}$ Ciénega de Flores on Mex Rt 85. Scaphiopus holbrookii: KU 90079: United States: Missouri: Dunklin: Kennett. MZUSP 5699: United States: Georgia: Savannaah. Scaphiopus hurterii: KU 187903: United States: Texas: Brazos: 1.6 km W College Station. Scarthyla goinorum: $\mathrm{CFBH}$ 24813: Brazil: Acre: Cruzeiro do Sul: Comunidade da Praia Grande. TG 2512: Brazil: Acre: Cruzeiro do Sul. Scinax granulatus: MACN 38218, MACN 38223: Argentina: Entre Ríos: Islas del Ibicuy: Old National Route 12, next to its intersection with the railway; MACN 41197: Argentina: Ciudad Autónoma de Buenos Aires: Reserva Ecológica Costanera Sur. Scutiger mammatus: FMNH 282284: China: Western Szechwan. Sooglossus sechellensis: UMMZ 146293: Seychelles: Mahe. Sooglossus thomasseti: UMMZ 144473: Seychelles: Mahe. Spea bombifrons: KU 79355: United States: Wyoming: Goshen: $7 \mathrm{mi}$ SW La Grange. MACN 39156: United States: New Mexico: Hidalgo: Cochise, 6 mi ESE Portal. Spea hammondii: CM 73782: United States: California: San Diego: Vicinity Lower Otay Lake, between Lake and Cal 94 on Otay Lake Road. Spea intermontana: KU 297558: United States: Arizona: Coconino. Spea multiplicata: KU 86656: Mexico: Morelos: $3.5 \mathrm{~km}$ W Cuautlixco. Sphaeno- 
rhynchus lacteus: ZUEC 5853: Brazil: Acre: Mâncio Lima: Lagoa da Cobra. Sphaerotheca breviceps: KU 200460: India: Orissa: Samblapur, Barpali. Staurois natator: KU 310055: Philippines: Dinagat Islands Province: Municipality of Loreto: Barangay Santiago, sitio Cambinlia (Sudlon). Stefania evansi: MZUSP 150734: Guyana: Potaro-Siparuni, Vecinity of Kuribrong River. Taudactylus diurnus: KU 179999: Australia: Queensland: Sunday Creek State Forest, Kilcoy Creek. Telmatobius oxycephalus: MACN 49004: Argentina: Jujuy: Tilcara. SB 16: Argentina: Jujuy: Tilcara: Garganta del Diablo, Río Huasamayo. Thoropa taophora: CFBH 12734: Brazil: São Paulo: Ubatuba: Cachoeira da Casa da Farinha, Picinguaba. Tomopterna delalandii: KU 207900: South Africa: Cape: vic Stellenbosch. Trachycephalus typhonius: CFBH 4969: Brazil: São Paulo: Presidente Epitácio: UHE - Porto Primavera; MACN 40540: Argentina: Santa Fe: Vera: Malabrigo. MACN 49595: Argentina: Salta: km 1765 RN 81, near Pluma de Pato. Triprion petasatus: KU 296231: Mexico: Yucatan: 3.6 km S. Dzitas. Uperodon systoma: KU 193677: India: Orissa: Puri: Bhubaneswar, Regional Research Lab Campus. Uperoleia laevigata: KU 180019: Australia: Queensland: Cooloola State Forest, Lake Cooloomera. Vitreorana uranoscopa: MACN 49000: Argentina: Misiones: San Pedro: Parque Provincial Esmeralda: Pozo Preto, Xenohyla truncata: CFBH 23533, MNRJ 33268: Brazil: Rio de Janeiro: Maricá: Restiga de Maricá. Xenopus borealis: KU 216442, KU 216458: Kenya: Maralal. Xenopus epitropicalis: KU 208520, KU 208527: Democratic Republic of Congo: Kinshasa. Xenopus laevis: KU 172920: Kenya: Rift Valley: Laikipia: $1.5 \mathrm{~km}$ W Naro Moru, Naro Moru River. Xenopus muelleri: KU 175228: Kenya: West Pokot: Kongelai: Suam River.

\section{APPENDIX 2}

\section{Comments on Phalanx Terminology}

Most contributions on anuran hand and foot musculature employ two terminologies when referring to the phalanges, one that we call "descriptive" (e.g., for a species with the typical four phalanges in digit IV of the foot, they are called: basal phalanx of digit IV, second phalanx of digit IV, third phalanx of digit IV, and distal phalanx of digit IV), and the other "technical" (e.g., for the same phalanges, the names are thus: Basal Phalanx IV, Antepenultimate Phalanx IV, Penultimate Phalanx IV, and Ultimate Phalanx IV). In some studies, the former terminology is employed (e.g., Dunlap, 1960), while in others, both are combined (e.g., Burton, 2004). Both terminologies are compared in the table below.

We employ here the descriptive terminology for two reasons. First, it is easier and less technical for the purpose of the present contribution, which is to describe to which specific phalanx each muscle attaches. Second, some anurans have digital reductions, and so a technical approach may obscure putative homologous insertions points. In this case, by employing the technical terminology, some of their homologies would require an additional, separate statement, as detailed below.

Exemplifying this second point, a putative homologous insertion on the same second phalanx of digit $\mathrm{V}$ of the foot could be described as (1) an insertion on Penultimate Phalanx $V$ in a species with no phalanx reduction (i.e., three phalanges present), and (2) an insertion on Ultimate Phalanx V (if the distal phalanx is lost). Furthermore, the reader would need to know the phalangeal formula of each species to interpret the specific phalanx referred to by the respective technical term, as shown in this example.

There are two drawbacks against the descriptive terminology, however. First, in species with digital reduction it poses problems similar to those encountered with technical terminology. However, these problems are restricted to the "distal phalan $\mathrm{x}$ " and are easily remedied by indi- 
cating the specific phalanx to which the term "distal" is referring when applied to species with digital reduction. A second problem with descriptive terminology is the economy, or lack thereof, given the number of characters and words required for clear expression. If the context is insufficient to infer the specific digit intended by "phalanx," more words may be required (e.g., if "distal phalanx" does not explicitly point to the correct digit, one might need to clarify by noting "distal phalanx of digit IV"). The table shows the equivalences between both terminologies.

\begin{tabular}{|c|c|c|c|}
\hline & & Descriptive & Technical \\
\hline \multicolumn{4}{|l|}{ Hand } \\
\hline \multirow{2}{*}{ Digit II } & Phalanx I & Basal phalanx of digit II & Basal Phalanx II \\
\hline & Phalanx II & Distal phalanx of digit II & Ultimate Phalanx II \\
\hline \multirow{2}{*}{ Digit III } & Phalanx I & Basal phalanx of digit III & Basal Phalanx III \\
\hline & Phalanx II & Distal phalanx of digit III & Ultimate Phalanx III \\
\hline \multirow{3}{*}{ Digit IV } & Phalanx I & Basal phalanx of digit IV & Basal Phalanx IV \\
\hline & Phalanx II & Second phalanx of digit IV & Penultimate Phalanx IV \\
\hline & Phalanx III & Distal phalanx of digit IV & Ultimate Phalanx IV \\
\hline \multirow{3}{*}{ Digit V } & Phalanx I & Basal phalanx of digit $\mathrm{V}$ & Basal Phalanx V \\
\hline & Phalanx II & Second phalanx of digit V & Penultimate Phalanx V \\
\hline & Phalanx III & Distal phalan $\mathrm{x}$ of digit $\mathrm{V}$ & Ultimate Phalanx V \\
\hline \multicolumn{4}{|l|}{ Foot } \\
\hline \multirow{2}{*}{ Digit I } & Phalanx I & Basal phalanx of digit I & Basal Phalanx I \\
\hline & Phalanx II & Distal phalanx of digit I & Ultimate Phalanx I \\
\hline \multirow{2}{*}{ Digit II } & Phalanx I & Basal phalanx of digit II & Basal Phalanx II \\
\hline & Phalanx II & Distal phalanx of digit II & Ultimate Phalanx II \\
\hline \multirow{3}{*}{ Digit III } & Phalanx I & Basal phalanx of digit III & Basal Phalanx III \\
\hline & Phalanx II & Second phalanx of digit III & Penultimate Phalanx III \\
\hline & Phalanx III & Distal phalanx of digit III & Ultimate Phalanx III \\
\hline \multirow{4}{*}{ Digit IV } & Phalanx I & Basal phalanx of digit IV & Basal Phalanx IV \\
\hline & Phalanx II & Second phalanx of digit IV & Antepenultimate Phalanx IV \\
\hline & Phalanx III & Third phalanx of digit IV & Penultimate Phalanx IV \\
\hline & Phalanx IV & Distal phalanx of digit IV & Ultimate Phalanx IV \\
\hline \multirow{3}{*}{ Digit V } & Phalanx I & Basal phalanx of digit $\mathrm{V}$ & Basal Phalanx V \\
\hline & Phalanx II & Second phalanx of digit $\mathrm{V}$ & Penultimate Phalanx V \\
\hline & Phalanx III & Distal phalanx of digit $\mathrm{V}$ & Ultimate Phalanx V \\
\hline
\end{tabular}




\section{APPENDIX 3}

\section{Anuran Hand and Foot Musculature: SYNONYM LIST}

As a general rule, we interpret authors' concept for each muscle based on their descriptions, figures, and/or explicit reference linking their concept to that of other authors. In the absence of these references, the names of the muscles mentioned are not included in the synonym list. For each muscle, the list of synonyms starts with the authors that employ the same name and concept as used in this study, and is denoted by "Same"; the remaining names of the list follow a chronological order on the first use of each alternative name.

Until the recent contributions of Diogo and associates, most literature followed the terminology of Gaupp (1896), with few modifications introduced by Dunlap (1960), Burton (1996, 2004), and Faivovich (2002). Considering this, we also include the most common synonym employed in the literature for each muscle group (e.g., the group of mm. transversi metacarporum of classical terminology is the group of the $\mathrm{mm}$. intermetacarpales sensu this study), except for the m. flexor accessorius of the foot (see below). This indication of common synonyms for the muscle groups allows a faster, albeit superficial, use of the table, being operationally similar to tables 2 and 4 of Diogo and Ziermann (2014).

A special mention is required for the $\mathrm{m}$. flexor accessorius (and synonyms) of the foot. This muscle is referred to a single muscle in the literature (m. flexor accessorius), or divided into a proximal and distal portion ( $\mathrm{m}$. flexor accessorius proximalis and distalis, respectively). There are species in which the $\mathrm{m}$. flexor accessorius distalis is absent while in others it is topologically present, although undifferentiated from the proximalis (Dunlap, 1960; this study). For this reason some authors mention only "flexor accessorius," which may imply one of the following situations: (1) the proximal and distal portions are present and distinct (although not specified); (2) both portions are present but undifferentiated; or (3) the distal portion is absent. Consid- ering this, and with the idea of recovering all the information from the literature in the synonym list, we include all the mentions of the term "flexor accessorius" (sensu lato), separately from the mentions of "flexor accessorius proximalis" and "flexor accessorius distalis."

Some muscle groups of some digits are composed of a medial and a lateral muscle (e.g., medial and lateral $\mathrm{m}$. lumbricalis brevis digiti $\mathrm{V}$ of the foot). We consider both muscles together for the elaboration of the synonym list. When some authors employ different names for the medial and lateral muscle of the group, we specify their correspondence with the medial and lateral muscle (sensu this study) in the discussion below, and not in the synonym list. The only exception is the m. flexor indicis brevis profundus, since the correspondence of its medial muscle to the $\mathrm{mm}$. flexores breves profundi group is controversial (see Discussion), and it is only tentatively adopted here.

Superscript "a" denotes muscles whose names and/or concepts require further discussion, and are addressed here. The use of "part." (abbreviation for partially) means that only a portion of the muscle named by an author is equivalent to our concept and name. As an example, the hand m. flexor teres digiti IV of Gaupp (1896) includes our $\mathrm{m}$. contrahentis digiti IV and $\mathrm{m}$. flexor minimus digiti IV. Consequently, we indicate it in the synonym list of both muscles as "flexor teres digiti IV (Gaupp, 1896: part.)." A question mark follows any muscle name whose identity is unclear as used by the author, and the reasons for our doubts are also provided here.

Superscript "b" denotes under the same muscle name reports from the literature of similar names, unless the authors' minimal terminological variation could affect its interpretation; it simplifies the lists and reduces redundancies. However, in this regard two situations require clarification: (1) reports that do not include within the name the specific digit to which the muscle belongs or attaches, and (2) reports that include the terms "digiti I" instead of "hallucis," and "digiti II" instead of "indicis." 
As an example of (1), if an author describes two "lumbricales breves" in the foot, one attaching to digit I (hallux) and the other to digit II, we include this report within the synonym list of the muscle named "lumbricalis brevis hallucis" and "lumbricalis brevis digiti II," respectively. Concerning (2), when an author employs "digiti I" instead of "hallucis," and digiti II instead of "indicis" for the construction of the name, we list it under both the name that includes the term "hallucis" and the other that includes "indicis." We employ these two shortcuts only if there are precedents of another author employing the names "lumbricalis brevis hallucis" and "lumbricalis brevis digiti II," or names including the term "hallucis" and "indicis." Otherwise (no author ever used the terms "lumbricalis brevis hallucis," "lumbricalis brevis digiti II", or names including "indicis" and "hallucis"), they are listed separately and as employed by the author in question. In this case, the names of the first example are listed separately as "lumbricalis brevis [of digit I]" and "lumbricalis brevis [of digit II]," denoting within brackets to which digit the generic name employed by the author refers to.

A few authors employ a numbering system I-IV for the digits of the hand (instead of the most common usage of II-V). To prevent confusion, we list the muscle names precisely as mentioned, avoiding the shortcuts mentioned above. Reports that differ only in the use of Roman or Arabic numbers (e.g., transversus metacarpi 1, 2, 3 instead of I, II, III, respectively) and those that do not includes "digiti" within the name (e.g., "flexor ossis metacarpi II" instead of "flexor ossis metacarpi digiti II") are considered fully equivalent for the purpose of the synonym list, and are not denoted with a superscript "b."

As detailed in Materials and Methods, the targeted muscles of the hand are those with origin and/or insertion on prepollex, metacarpals and/ or phalanges, except for the $\mathrm{m}$. contrahentis caput longus distalis (which joins the ulnare and distal carpals). Besides this exception, two muscles of the forearm do not attach to these predefined regions and are mentioned across the study: the $\mathrm{m}$. extensor carpi radialis and $\mathrm{m}$. extensor carpi ulnaris. For the latter, there is no change in its concept and name between the classical and new terminology. Distinctly, the identity of the different portions assigned to the $\mathrm{m}$. extensor carpi radialis is controversial, and we follow Diogo et al. (2018: 459-460) for its name and concept.

Two studies were not included in the synonym list: Hoffman (1878) and Perrin (1891). The former has many descriptive inaccuracies (Perrin, 1891, 1892), it is considered to be a compilation of previous studies (Perrin, 1892: 5), and it went almost unmentioned in the literature of anuran hand and foot musculature. The study of Perrin (1891) does not assign names to the muscles, and it is mostly restricted to review, compare, and indicate descriptive inaccuracies in the contributions of Dugès (1834), Hoffmann (1878), and Ecker (1864).

The table is arranged in the following order: ventral forearm muscles included in this study, ventral hand, dorsal forearm/hand, ventral leg, ventral foot, and dorsal leg/foot. Except for the ventral forearm and ventral leg, muscles follow the order of plates 2-5 (from superficial to deep layers and, within each layer, from proximal to distal and from radial/tibial to ulnar/fibular side. 
APPENDIX 3 continued

\begin{tabular}{l}
\hline Terminology used in this study \\
\hline VENTRAL FOREARM
\end{tabular}

\begin{tabular}{ll}
\hline & Same (Ecker, 1864; Abdala and Diogo, 2010; Diogo and Abdala, 2010; Diogo and Zier- \\
& mann, 2014; Diogo et al., 2018; Fratani et al., 2018, 2020; Soliz et al., 2020); épitrochlo- \\
& sous-phalangettien (Dugès, 1834); palmaris longus (Gaupp, 1895, 1896; Kändler, 1924; \\
& Liem, 1970, 1973; Liem and Hosmer, 1973; Davies and Burton, 1982; Burton, 1983; Oka «t \\
& al., 1984; Duellman and Trueb, 1986; Burton, 1996, 1998a; Faivovich, 2002; Faivovich et al., \\
& 2005; Manzano et al., 2008; Salgar et al., 2009; Hoyos et al., 2014; Hoyos and Salgar, 2016); \\
& flexor primordialis communis (Ribbing, 1907: part. ${ }^{2}, 1911$ : part. ${ }^{2}$ ); flexor digitorum com- \\
& munis longus (Manzano et al., 2008; Oliveira-Lagôa et al., 2019); flexor digitorum longus \\
& (Abdala et al., 2018; Oliveira-Lagôa et al., 2019) \\
\hline
\end{tabular}

Same (Ribbing, 1907; 1911; Abdala and Diogo, 2010; Diogo and Abdala, 2010; Diogo and Ziermann, 2014; Dingo et al., 2018); cubito-palmaire (Dugès, 1834); palmaris brevis (Ecker, 1864;

Flexor accessorius Salgar et al., 2009; Hoyos et al., 2014; Hoyos and Salgar, 2016); palmaris profundus (Gaupp. 1895, 1896; Kändler, 1924; Liem, 1970; Davies and Burton, 1982; Burton, 1983; Duellman and Trueb, 1986; Burton, 1996, 1998a; Faivovich, 2002; Manzano et al., 2008; Salgar et al., 2009);

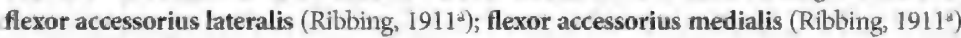

Same (Abdala and Diogo, 2010; Diogo and Abdala, 2010; Diogo and Ziermann, 2014; Diogo et

Pronator quadratus Kändler, 1924; Davies and Burton, 1982; Burton, 1983; Duellman and Trueb, 1986; Burton, 1996, al., 2018); cubito-pollicien (Dugès, 1834); abductor pollicis (Ecker, 1864; Gaupp, 1895, 1896; 1998a; Manzano et al,, 2008; Salgar et al., 2009; Hoyos et al., 2014; Hoyos and Salgar, 2016); pronator profundus (Ribbing, 1907, 1911); adductor pollicis (Burton, 1983: part. ${ }^{2}$ )

\begin{tabular}{|c|c|}
\hline VENTRAL HAND & \\
\hline Superficial cutaneous tendons & This study \\
\hline Tendines superficiales group & No significant change \\
\hline Tendo superficialis indicis & 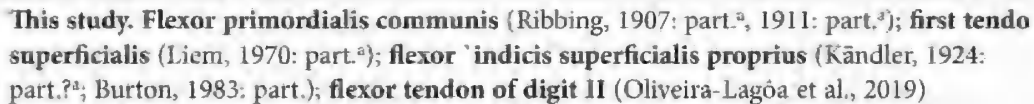 \\
\hline
\end{tabular}
part ? ${ }^{\text {s; }}$ Burton, 1983: part.); flexor tendon of digit 11 (Oliveira-Lagóa et al., 2019)

Same (Gaupp, 1895' 1896 ; Burton, 1983b, 1996, 1998a, 1998b; Faivovich, 2002; Manzano et al., 2008b; Salgar et al., 2009b; Hoyos et al., 2014; Barrionuevo, 2017b); épitrochlo-sousphalangettien (Dugès, 1834: part. ${ }^{\text {a)}) ; ~ f l e x o r ~ d i g i t i ~ I I I ~ l o n g u s ~ s, ~ s u b l i m i s ~(E c k e r, ~ 1864: ~}$

Tendo superficialis digiti III part. ${ }^{2}$; flexor primordialis communis (Ribbing, 1907: part ${ }^{2}, 1911$ : part. ${ }^{x}$ ); second tendo superficialis (Liem, 1970); tendo superficialis of second finger (Liem and Hosmer, 1973); superficial tendon III (Manzano et al., 2008); flexor tendon of digit III (Manzano et al,, 2008; Fratani et al., 2018; Oliveira-Lagùa et al., 2019)

Same (Gaupp, 1895 ; Burton, 1996 , 1998a; Faivovich, 2002; Manzano et al., 2007 ; Hoyos et al., 2014); épitrochlo-sous-phalangettien (Dugès, 1834: part. ${ }^{a}$ ); flexor digiti IV longus s. sublimis (Ecket; 1864: part."); tendo superficialis Aponeurosis palmaris pro digiti IV (Gaupp, 1896; Davies and Burton, 1982; Burton, 1983, 1998a; Salgar et al., 2009; Hoyos et Tendo superficialis digiti IV al., 2014); flexor primordialis communis (Ribbing, 1907: part. ${ }^{2}$ 1911: part. ${ }^{2}$ ); third tendo superficialis (Liem, 1970); tendo superficialis of third finger (Liem and Hosmer, 1973; Hanna and Barnes, 1991); superficial tendon IV (Manzano et al,, 2008); flexor tendon of digit IV (Manzano et al., 2008; Dos Santos et al.s 2014; Abdala et aJ., 2018; Fratani et al., 2018; Oliveira-Lagóa et al., 2019)

Same (Gaupp, 1895 ; Burton, 1996 , 1998a; Faivovich, 2002; Hoyos et al., 2014); épitrochlosous-phalangettien (Dugès, 1834: part. ${ }^{2}$ ); flexor digiti V longus s. sublimis (Ecker, 1864: part."); tendo superficialis Aponeurosis palmaris pro digiti V (Gaupp, 1896; Davies and BurTendo superficialis digiti V ton, 1982; Burton, 1983, 1998a; Hoyos and Salgar, 2016); flexor primordialis communis (Ribbing, 1907: part. ${ }^{a}, 1911$ : part. ${ }^{3}$ ); fourth tendo superficialis (Liem, 1970); tendo superficialis of fourth finger (Liem and Hosmer, 1973): superficial tendon V (Manzano et al., 2008); flexor tendon of digit V (Manzano et al., 2008; Fratani et al., 2018; Oliveira-Lagôa et al., 2019) 
APPENDIX 3 continued

\begin{tabular}{|c|c|}
\hline Terminology used in this study & Synonyms \\
\hline $\begin{array}{l}\text { Flexor indicis superficialis } \\
\text { proprius }\end{array}$ & 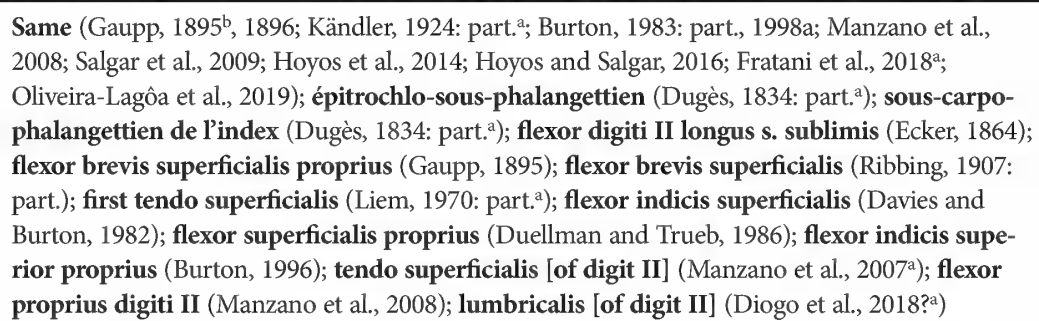 \\
\hline
\end{tabular}

Same (Gaupp, 1895 ; Burton, 1998a ${ }^{b}, 1998 b^{b}$; Manzano et al., 2008; Salgar et al., 2009; Hoyos et al., 2014; Fratani et al., 2018 ); sous-carpo-phalangettien du médius (Dugès,

Caput profundum digiti III 1834); flexor digiti III longus s. sublimis (Ecker, 1864: part. ${ }^{\text {) }}$; caput profundum tendonis superficialis digiti III (Gaupp, 1896; Kändler, 1924; Davies and Burton, 1982?a,b; Burton, 1983, 1998a); flexor brevis superficialis (Ribbing, 1907: part. $^{\text {b }}$ ); caput profundus III (Burton, 1996); lumbricalis [of digit III] (Diogo et al., 2018?a)

\begin{tabular}{|c|c|}
\hline Lumbricales longi group & No change \\
\hline Lumbricalis longus digiti III & $\begin{array}{l}\text { Same (Burton, 1998a, 1998b }{ }^{\text {; }} \text { Barrionuevo, 2017a); lumbricalis brevis digiti III (Burton, } \\
\text { 1998a: part. }{ }^{\text {a }} \text {; lumbricalis longi [of second digit] (Aguilar and Valencia, 2009: } \text { part. }^{\mathrm{a}} \text { ) }\end{array}$ \\
\hline Lumbricalis longus digiti IV & 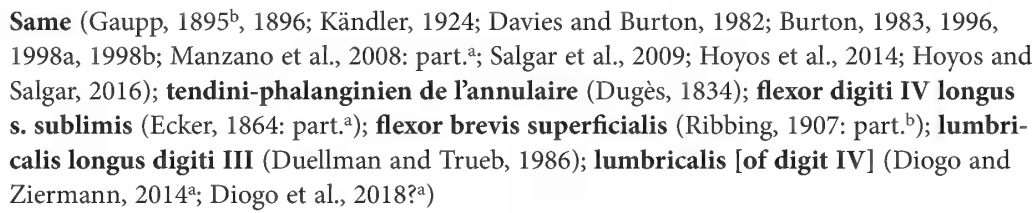 \\
\hline
\end{tabular}

Same (Gaupp, 1895하 1896; Kändler, 1924; Davies and Burton, 1982; Burton, 1983, 1996, 1998a; Manzano et al., 2008 ; Hoyos et al., 2014); tendini-phalanginien du digitule

Lumbricalis longus digiti $\mathrm{V}$ (Dugès, 1834); flexor digiti V longus s. sublimis (Ecker, 1864: part. ${ }^{a}$ ); flexor brevis superficialis (Ribbing, 1907: part.b); lumbricalis longus digiti IV (Duellman and Trueb, 1986 ${ }^{\text {b); }}$ lumbricalis [of digit V] (Diogo and Ziermann, 2014a; Diogo et al., 2018?a)

Same (Abdala and Diogo, 2010; Diogo and Abdala, 2010; Diogo et al., 2018; Fratani et al., 2018; Oliveira-Lagôa et al., 2019); aponeurosis palmaris (Ecker, 1864; Gaupp, 1895, 1896; Kändler, 1924; Liem, 1970, 1973; Liem and Hosmer, 1973; Duellman and Trueb, 1986; Bur-

Flexor plate ton, 1996, 1998a, 1998b; Faivovich, 2002; Salgar et al., 2009; Hoyos et al., 2014; Barrionuevo, 2017; Araujo-Vieira et al., 2019); flexor primordialis communis (Ribbing, 1907: part. ${ }^{a}, 1911$ part. ${ }^{\text {) }}$; palmar aponeurosis (Davies and Burton, 1982; Burton, 1983, 1986, 1998b; Manzano et al., 2008; Diogo and Ziermann, 2014); superficial aponeurosis (Burton, 1983?)

\begin{tabular}{ll}
\hline Burton's ligament & Same (Faivovich, 2002; Araujo-Vieira et al., 2019) \\
\hline Lumbricales breves group & No change \\
\hline
\end{tabular}

Same (Gaupp, 1895하 1896; Kändler, 1924; Davies and Burton, 1982: part.?a; Burton, 1983?a, 1996, 1998a; Salgar et al., 2009; Hoyos et al., 2014); tendini-phalangien de l'index (Dugès,

Lumbricalis brevis indicis 1834); flexor digiti II brevis s. profundus (Ecker, 1864); flexor brevis superficialis [of digit II] (Ribbing, 1907; Diogo and Ziermann, 2014ª; Diogo et al., 2018?a); m. flexor indicis superficialis proprius (Kändler, 1924: part.?a)

Same (Gaupp, 1895, 1896; Kändler, 1924; Davies and Burton, 1982; Burton, 1983, 1996, 1998a, 1998b; Manzano et al., 2008; Salgar et al., 2009; Hoyos et al., 2014; Barrionuevo, 2017ª; Garg and Biju, 2019a); tendini-phalangien du médius (Dugès, 1834); flexores digiti

Lumbricalis brevis digiti III III breves s. profundi I, II (Ecker, 1864 a); interosseus volaris [of digit III] (Ecker, 1864?a); flexor brevis superficialis [of digit III] (Ribbing, 1907, 1911; Diogo and Ziermann, 2014; Diogo et al., 2018?a); caput profundum tendonis superficialis digiti III (Davies and Burton, 1982 a); lumbricalis longi [of digit III] (Aguilar and Valencia, 2009: part. ${ }^{a}$ ) 
APPENDIX 3 continued

\begin{tabular}{|c|c|}
\hline Terminology used in this study & Synonyms \\
\hline Lumbricalis brevis digiti IV & 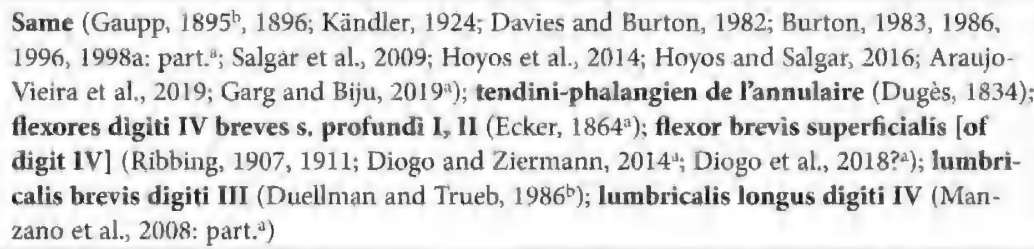 \\
\hline
\end{tabular}

Same (Gaupp, 1895, 1896; Kändler, 1924; Davies and Burton, 1982; Burton, 1983: part," 1996. 1998a; Faivovich, 2002; Manzano et al., 2008; Salgar et al., 2009; Hoyos et al., 2014; Araujo-Vieira et al,, 2019); sous-carpo-métacarpo-phalangien du digitule (Dugès,

Lumbricalis brevis digiti $\mathrm{V} \quad$ 1834? $)$; tendini-phalangien du digitule (Dugès, 1834); flexor digiti V brevis (Ecker, 1864 $\left.{ }^{3}\right)$; abductor primus digiti V (Ecker, 1864?"); flexor brevis superficialis [of digit V] (Ribbing, 1907; Diogo and Ziermann, 2014ª Diogo et al., 2018? ${ }^{\mathrm{a}}$ ); lumbricalis brevis digiti IV (Duellman and Trueb, 1986 $)$; lumbricalis longus digiti V (Faivovich, 2002

\begin{tabular}{|c|c|}
\hline Contrahentes group & $\begin{array}{l}\text { No unified name across the literature; digit II: flexor teres; digit IV: flexor teres, head } \\
\text { from distal carpals; digit V: adductor proprius }\end{array}$ \\
\hline Contrahentis indicis & 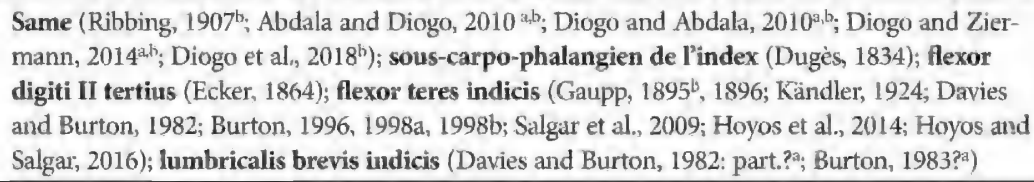 \\
\hline Contrahentis digiti III & 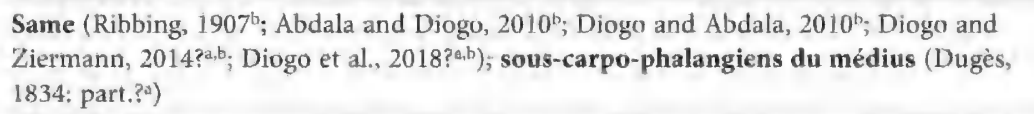 \\
\hline Contrahentis digiti IV & 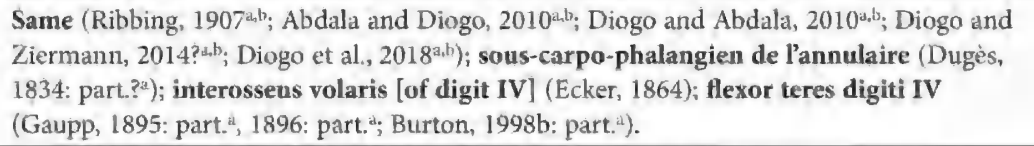 \\
\hline Contrahentis digiti $\mathrm{V}$ & 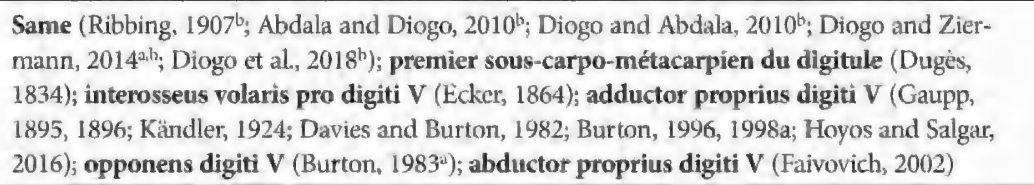 \\
\hline $\begin{array}{l}\text { Contrahentis caput longus } \\
\text { distalis. }\end{array}$ & $\begin{array}{l}\text { This study. Intercarpalis (Gaupp, 1895, 1896; Kändler, 1924; Davies and Burton, 1982; Burton, } \\
\text { 1983, 1996, 1998a: Salgar et al., 2009); caput longun muscularum contrahentium (Ribbing, } \\
\text { 1907: part.3, 1911: part.a); contrahentium caput longum (Abdala and Diogo, 2010: part.; Diogo } \\
\text { and Abdala, 2010: part,; Diogo and Ziermann, 2014: part.; Diogo et al,, 2018; part.) }\end{array}$ \\
\hline Adductor pollicis & 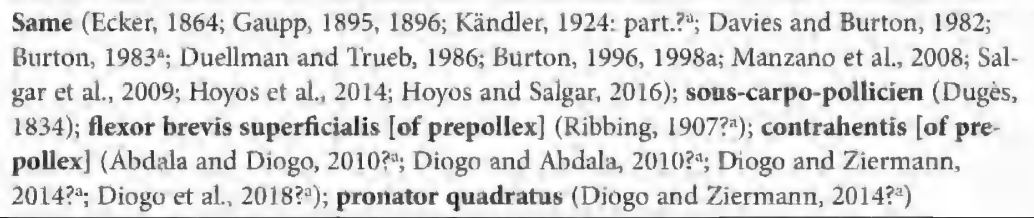 \\
\hline Flexores breves profundi group & Flexores ossum metacarporum (digits III-IV); opponens (digits II and V) \\
\hline $\begin{array}{l}\text { Flexor indicis brevis profundus, } \\
\text { medial muscle }\end{array}$ & 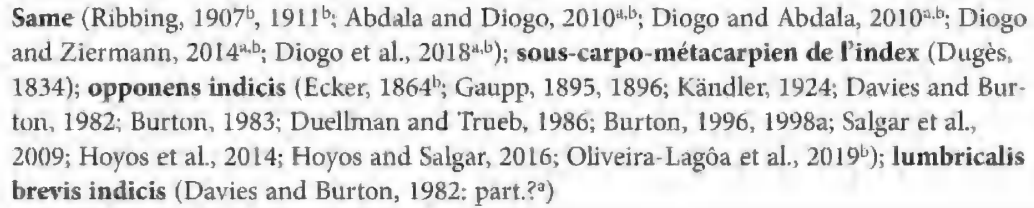 \\
\hline
\end{tabular}


APPENDIX 3 continued

\begin{tabular}{|c|c|}
\hline Terminology used in this study & Synonyms \\
\hline $\begin{array}{l}\text { Flexor indicis brevis profundus, } \\
\text { lateral muscle }\end{array}$ & This study \\
\hline $\begin{array}{l}\text { Flexor brevis profundus digiti } \\
\text { III }\end{array}$ & 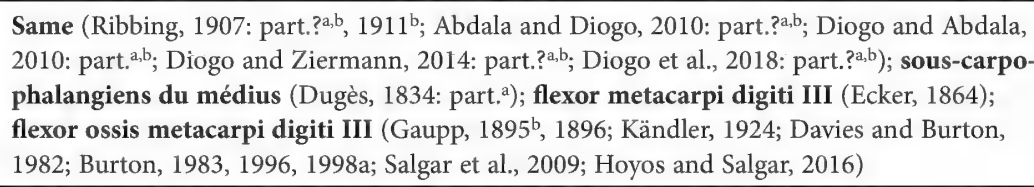 \\
\hline $\begin{array}{l}\text { Flexor brevis profundus digiti } \\
\text { IV }\end{array}$ & 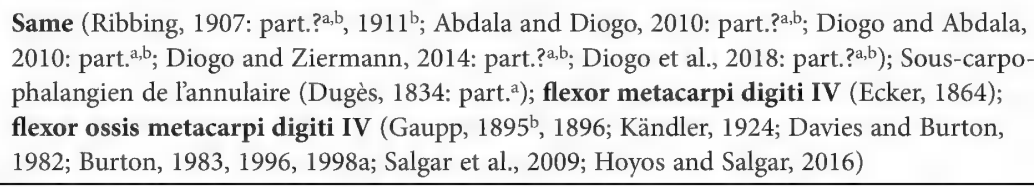 \\
\hline $\begin{array}{l}\text { Flexor brevis profundus digiti } \\
\text { V }\end{array}$ & $\begin{array}{l}\text { Same (Ribbing, 1907: part.? ?a,b, } 1911 \text { ?a,b; Abdala and Diogo, 2010: part.?a.b; Diogo and } \\
\text { Abdala, 2010: part.?a, ; Diogo and Ziermann, 2014: part.? ?a,b; Diogo et al., 2018: part.? }{ }^{\mathrm{a}, \mathrm{b}} \text { ); } \\
\text { deuxième sous-carpo-métacarpien du digitule (Dugès, 1834); opponens digiti V (Ecker, } \\
\text { 1864; Gaupp, 1895, 1896; Kändler, 1924; Davies and Burton, 1982; Burton, 1996, 1998a; } \\
\text { Salgar et al., 2009?a); lumbricalis brevis digiti V (Burton, 1983: part. }{ }^{\mathrm{a}} \text { ) }\end{array}$ \\
\hline Abductor digiti minimi & $\begin{array}{l}\text { Same (Abdala and Diogo, 2010; Diogo and Abdala, 2010; Diogo and Ziermann, 2014; } \\
\text { Diogo et al., 2018); sous-pyro-pré-métacarpien du digitule (Dugès, 1834?ª); abductor } \\
\text { primus digiti V (Ecker, 1864?a; Gaupp, 1895, 1896; Kändler, 1924; Davies and Burton, } \\
\text { 1982: part.?a; Burton, 1983?a, 1996, 1998a; Salgar et al., 2009; Hoyos and Salgar, 2016); } \\
\text { flexor brevis profundus [of digit V] (Ribbing, 1907?a); flexor brevis superficialis [of digit } \\
\text { V] (Ribbing, 1907?a); abductor primus digiti IV (Duellman and Trueb, 1986) }\end{array}$ \\
\hline Abductor secundus digiti $\mathrm{V}$ & $\begin{array}{l}\text { Same (Ecker, 1864' Gaupp, 1895, 1896; Kändler, 1924: part.?a; Davies and Burton, 1982: } \\
\text { part.?a; Burton, 1983?a , 1996, 1998a; Manzano et al., 2008; Salgar et al., 2009; Hoyos and } \\
\text { Salgar, 2016); sous-pyro-post-métacarpien du digitule (Dugès, 1834); abductor digiti V } \\
\text { (Ribbing, 1907, 1911?a); abductor secundus digiti IV (Duellman and Trueb, 1986); flexor } \\
\text { brevis profundus [of digit V] (Abdala and Diogo, 2010?a; Diogo and Abdala, 2010?a; } \\
\text { Diogo et al., 2018?a); abductor digiti minimi (Diogo and Ziermann, 2014?a) }\end{array}$ \\
\hline
\end{tabular}

Flexores digitorum minimi group

Flexores teretes with exception of digit II

\begin{tabular}{|c|c|}
\hline Flexor minimus indicis & Same (Ribbing, $1911^{b}$ ) \\
\hline Flexor minimus digiti III & $\begin{array}{l}\text { Same (Ribbing, 1907 }{ }^{\mathrm{a}, \mathrm{b}}, 1911 \text {; Diogo and Ziermann, 2014 } \\
\text { carpo-phalangiens du médius (Dugès, 1834: part.?a); flexor teres digiti III (Gaupp, 1895, } \\
\text { 1896; Kändler, 1924; Davies and Burton, 1982; Burton, 1983, 1996, 1998a, 1998b; Blotto et } \\
\text { al., 2013; Hoyos and Salgar, 2016; Garg and Biju, 2019); flexor brevis profundus [of digit } \\
\text { III] (Abdala and Diogo, 2010: part.?a; Diogo and Abdala, 2010: part. ; Diogo and Zier- } \\
\text { mann, 2014: part.? a; Diogo et al., 2018: part.? }{ }^{\text {a })}\end{array}$ \\
\hline
\end{tabular}

Same (Ribbing, 1907ab, 1911, Diogo and Ziermann, 2014 ${ }^{\text {b }}$ Diogo et al., 2018 ); flexor teres

digiti IV (Gaupp, 1895: part. a, 1896: part. a, Kändler, 1924; Davies and Burton, 1982; Burton, 1983, 1996, 1998a, 1998b: part. a; Salgar et al., 2009; Blotto et al., 2013; Hoyos and Salgar, 2016); flexor brevis profundus [of digit IV] (Abdala aºnd Diogo, 2010: part.?; Diogo and Abdala, 2010: part.a; Diogo and Ziermann, 2014: part.?a; Diogo et al., 2018: part.?a)

Same (Ribbing, 1907 a,b, 1911; Diogo and Ziermann, 2014 ; Diogo et al., 2018 ); flexor teres

$\begin{array}{ll}\text { Flexor minimus digiti V } & \text { digiti V (Gaupp, 1895, 1896; Davies and Burton, 1982; Burton, 1983, 1996, 1998a, 1998b; }\end{array}$ (Abdala and Diogo, 2010: part ?a. Diogo and Abdala, 2010: part a. Diogo and Ziermann, 2014: part.?a; Diogo et al., 2018: part.?a)

Intermetacarpales group Transversi metacarporum 
APPENDIX 3 continued

\begin{tabular}{|c|c|}
\hline Terminology used in this study & Synonyms \\
\hline Intermetacarpalis I & $\begin{array}{l}\text { Same (Abdala and Diogo, 2010 } \text {; Diogo and Abdala, 2010 } \text {; Diogo and Ziermann, 2014 } \\
\text { Diogo et al., 2018 } \\
\text { (Ecker, 1864); métacarpo-métacarpien de l'index (Dugès, 1834); adductor digiti II } \\
\text { Burton, 1982; Burton, 1983a, 1996, 1998a, 1998b; Salgar et al., 2009; Blotto et al., 2013; } \\
\text { Hoyos and Salgar, 2016; Garg and Biju, 2019); interosseus (Ribbing, 1907, 1911) }\end{array}$ \\
\hline
\end{tabular}

Hoyos and Salgar, 2016; Garg and Biju, 2019); interosseus (Ribbing, 1907, 1911)

Intermetacarpalis II

(Abdala and Diogo, 2010 ; Diogo and Abdala, 2010'; Diogo and Ziermann, 2014 Diogo et al., 2018 ); transversus metacarpi II (Ecker, 1864'; Gaupp, 1895 , 1896; Kändler, 1924; Davies and Burton, 1982; Burton, 1983, 1996, 1998a, 1998b; Salgar et al., 2009; Blotto et al., 2013; Hoyos and Salgar, 2016); interosseus (Ribbing, 1907, 1911)

\begin{tabular}{|c|c|}
\hline Intermetacarpalis III & $\begin{array}{l}\text { Same (Abdala and Diogo, } 2010^{\text {b}} \text {; Diogo and Abdala, 2010 } \text {; Diogo and Ziermann, 2014 } \\
\text { Diogo et al., 2018 } \\
\text { 1924; Davies and Burton, 1982; Burton, 1983, 1996, 1998a, 1998b; Salgar et al., 2009; Hoyos } \\
\text { and Salgar, 2016); interosseus (Ribbing, 1907, 1911) }\end{array}$ \\
\hline Interphalangei group & Interphalangeales \\
\hline Interphalangeus indicis & This study \\
\hline Interphalangeus digiti III & This study \\
\hline
\end{tabular}

Same (Ribbing, 1907b 1911 ?a,b; Diogo and Ziermann, 2014; Diogo et al., 2018); phalangophalanginien de l'annulaire (Dugès, 1834); flexor proprius phalangum digiti IV (Ecker, Interphalangeus digiti IV 1864); interphalangealis digiti IV (Gaupp, 1895', 1896; Kändler, 1924; Davies and Burton, 1982; Burton, 1983, 1996, 1998a); flexor interphalangealis [of digit IV] (Gaupp, 1895'); interphalangealis digiti III (Duellman and Trueb, 1986 ${ }^{\mathrm{b}}$ )

Same (Ribbing, 1907b, $1911^{\mathrm{a} b \mathrm{~b}}$; Diogo and Ziermann, 2014; Diogo et al., 2018); phalango-

Interphalangeus digiti $\mathrm{V}$ phalanginien du digitule (Dugès, 1834); flexor proprius phalangum digiti $\mathbf{V}$ (Ecker, 1864); interphalangealis digiti V (Gaupp, 1895' , 1896; Kändler, 1924; Davies and Burton, 1982; Burton, 1983, 1996, 1998a); flexor interphalangealis [of digit V] (Gaupp, 1895b)

DORSAL FOREARM/HAND

Same (Abdala and Diogo, 2010; Diogo and Abdala, 2010; Diogo and Ziermann, 2014; Diogo et al., 2018; Soliz et al., 2020); huméro-sus-digital (Dugès, 1834); extensor digitorum communis longus (Ecker, 1864; Gaupp, 1895, 1896; Kändler, 1924; Davies and Bur-

Extensor digitorum ton, 1982; Burton, 1983; Oka et al., 1984; Duellman and Trueb, 1986; Burton, 1996, 1998a; Faivovich, 2002; Manzano et al., 2008; Salgar et al., 2009; Hoyos et al., 2014; Hoyos and Salgar, 2016; Araujo-Vieira et al., 2019); extensor digitorum communis (Ribbing, 1907: part. $^{\text {a }, ~ 1911: ~ p a r t . ~ a ; ~ B u r t o n, ~ 1983) ; ~ h u m e r o d o r s a l i s ~(H a i n e s, ~ 1939 ; ~ L i e m, ~ 1970, ~ 1973 ; ~ I n g e r, ~}$ 1972; Liem and Hosmer, 1973; Fabrezi et al., 2017)

Same (Abdala and Diogo, 2010; Diogo and Abdala, 2010; Diogo and Ziermann, 2014; Diogo et al., 2015; Diogo et al., 2018); cubito-métacarpien (Dugès, 1834); abductor indicis longus (Ecker, 1864 ; Gaupp, 1896; Kändler, 1924: part. a; Davies and Burton, 1982;

Abductor pollicis longus Burton, 1983; Oka et al., 1984; Duellman and Trueb, 1986; Burton, 1996, 1998a; Manzano et al., 2008'a; Salgar et al., 2009; Hoyos and Salgar, 2016); extensor digitorum communis

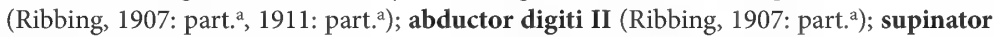
manus (Haines, 1939; Liem, 1970, 1973; Inger, 1972; Liem and Hosmer, 1973)

Extensores breves superficiales No significant change
group

Same (Gaupp, 1895º, 1896: part.? ?a; Kändler, 1924: part.? ?a; Ribbing, 1907: parta, , , 1911: part.a,b; Haines, 1939 a,b; Liem, 1970a,b; Davies and Burton, 1982; Burton, 1983; Duellman

Extensor indicis brevis superficialis and Trueb, 1986a; Burton, 1996, 1998a: part.?a; Manzano et al., 2008; Salgar et al., 2009; Diogo and Ziermann, 2014 ; Hoyos and Salgar, 2016); cubito-radio-sus-phalangien de l'index (Dugès, 1834); extensor digiti II proprius longus (Ecker, 1864: part. ${ }^{\mathrm{a}}$ ); abductor indicis longus (Kändler, 1924a) 
APPENDIX 3 continued

\begin{tabular}{|c|c|}
\hline Terminology used in this study & Synonyms \\
\hline $\begin{array}{l}\text { Extensor brevis superficialis } \\
\text { digiti III }\end{array}$ & $\begin{array}{l}\text { Same (Gaupp, 1895, 1896; Kändler, 1924; Ribbing, 1907: part. a,b, 1911: part. }{ }^{\text {ablb; }} \text { Haines, } \\
\text { 1939a,b; Davies and Burton, 1982; Burton, 1983, 1996, 1998a; Faivovich, 2002; Salgar et al., } \\
\text { 2009; Diogo and Ziermann, 2014 }{ }^{\text {b }} \text {; Hoyos and Salgar, 2016); sus-pyro-phalangien du } \\
\text { médius (Dugès, 1834); sus-pyro-phalangettien du médius (Dugès, 1834); extensor digito- } \\
\text { rum communis brevis (Ecker, 1864); externus digitorum brevis superficialis (Liem and } \\
\text { Hosmer, 1973) }\end{array}$ \\
\hline $\begin{array}{l}\text { Extensor brevis superficialis } \\
\text { digiti IV }\end{array}$ & 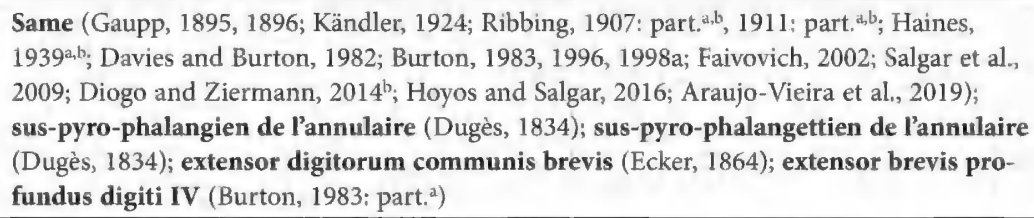 \\
\hline $\begin{array}{l}\text { Extensor brevis superficialis } \\
\text { digiti V }\end{array}$ & 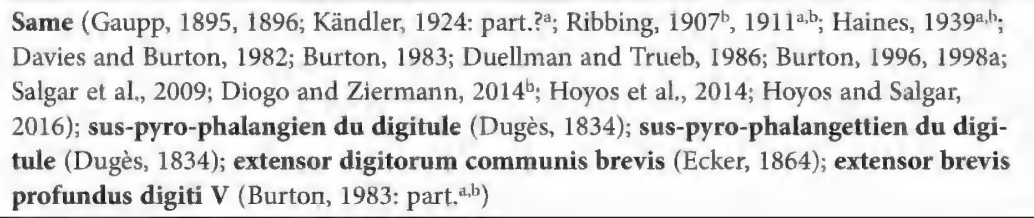 \\
\hline Extensores breves medii group & No change \\
\hline
\end{tabular}

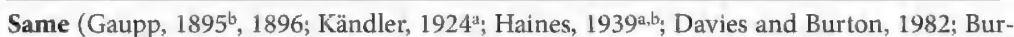
ton, 1983' ; Duellman and Trueb, 1986; Burton, 1996, 1998a; Salgar et al., 2009; Diogo and

Extensor indicis brevis medius Ziermann, 2014 ${ }^{\mathrm{b}}$; Hoyos and Salgar, 2016); sus-luno-phalangettien de l'index (Dugès, 1834); extensor digiti II proprius brevis (Ecker, 1864: part," ); extensor indicis brevis superficialis (Ribbing, 1907: part.ab, 1911: part.?a,b; Kändler, 1924: part.?a)

Same (Gaupp, 1895, 1896; Kändler, 1924; Haines, 1939, , bavies and Burton, 1982; Burton,

Extensor brevis medius digiti III 1983. Duellman and Trueb, 1986'b; Burton, 1996, 1998a; Salgar et al., 2009; Diogo and Zie mann, 2014 ${ }^{\text {b }}$, Hoyos and Salgar, 2016); sus-luno-phalangettien du médius (Dugès, 1834); extensor digiti III proprius (Ecker, 1864: part. ${ }^{\mathrm{a}}$ ); extensor brevis superficialis [of digit III] (Ribbing, 1907: part. ${ }_{2}$ 1911: part. ${ }^{2}$ )

Same (Gaupp, 1895ㅁ, 1896; Kändler, 1924; Haines, 1939 ton, 1983, 1996, 1998a; Faivovich, 2002; Faivovich et al., 2005; Salgar et al., 2009; Diogo and Ziermann, 2014 ; Hoyos and Salgar, 2016); sus-carpo-phalangettien de l'annulaire

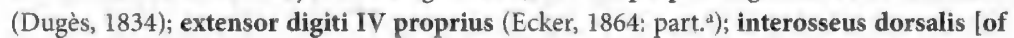
digit IV] (Ecker, 1864: part.?a); extensor brevis superficialis [of digit IV] (Ribbing, 1907: part.", 1911: part."

Same (Gaupp, 1895, 1896; Kändler, 1924; Davies and Burton, 1982; Burton, 1983a; Duellman and Trueb, 1986; Burton, 1996, 1998a; Salgar et al., 2009); sus-luno-métacarpien de l'index (Dugès, 1834); abductor digiti II brevis (Ecker, 1864); abductor digiti II (Ribbing, 1907: part. ${ }^{\text {) }}$

Same (Davies and Burton, 1982; Burton, 1983, 1996, 1998a; Salgar et al., 2009; Hoyos et al., 2014; Hoyos and Salgar, 2016); abductor secundus digiti V? (Kändler, 1924a)

Abductor brevis digiti $\mathrm{V}$

Extensores breves profundi

Dorsometacarpales proximales group

This study. Sus-métacarpo-phalango-phalangettien de l'index (Dugès, 1834" ${ }^{\text {in }}$; extensor digiti II proprius longus (Ecker, 1864: part. ${ }^{a}$ ); extensor dig. II proprius brevis (Ecker, 1864: part. ${ }^{\mathrm{A}}$ ); extensor indicis brevis profundus (Gaupp, 1895 ${ }^{\text {b }}$, 1896; Ribbing, 1907 ${ }^{\text {, }}$, 1911 b : Davies and Burton, 1982; Burton, 1983, 1996, 1998a, 1998c; Fabrezi and Langone, 2000: part. a.b; Manzano et al., 2007b; Salgar et al., 2009; Hoyos and Salgar, 2016; AraujoVieira et al,, 2019 $9^{b}$ ); dorsometacarpalis [of digit II] (Haines, 1939"s Diogo and Ziermann, 2014; Diogo et al., 2018); extensor brevis distalis digiti II (Faivovich, 2002: part. ${ }^{\text {a }}$ ) 
APPENDIX 3 continued

\begin{tabular}{|c|c|}
\hline Terminology used in this study & Synonyms \\
\hline $\begin{array}{l}\text { Dorsometacarpalis proximalis } \\
\text { digiti III }\end{array}$ & 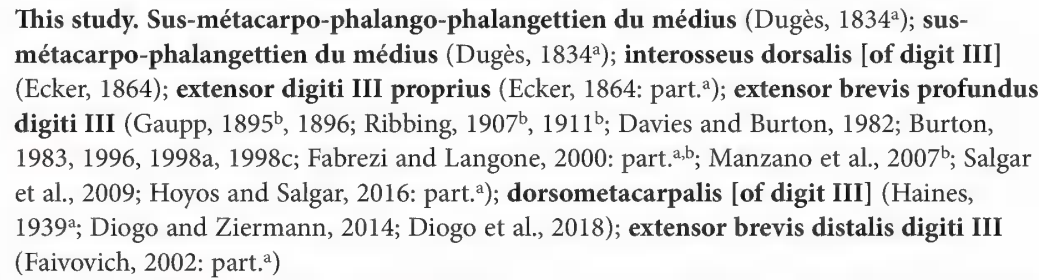 \\
\hline
\end{tabular}

(Faivovich, 2002: part. $^{\text {a) }}$

This study. Sus-métacarpo-phalangettien de l'annulaire (Dugès, 1834a); sus-métacarpophalango-phalangettien de l'annulaire (Dugès, 1834 ${ }^{\mathrm{a}}$ ); interosseus dorsalis [of digit IV] (Ecker, 1864: part. ${ }^{a}$ ); extensor digiti IV proprius (Ecker, 1864: part. ${ }^{\mathrm{a}}$ ); extensor brevis

Dorsometacarpalis proximalis profundus digiti IV (Gaupp, 1895 , 1896; Kändler, 1924; Ribbing, 1907, $1911^{\mathrm{b}}$; Davies and digiti IV Burton, 1982; Burton, 1983: part.a; Duellman and Trueb, 1986'b; Hanna and Barnes, 1991?a,b; Burton, 1996, 1998a, 1998c; Fabrezi and Langone, 2000: part. ab; Manzano et al., 2007 ; Salgar et al., 2009; Hoyos and Salgar, 2016: part. ${ }^{\text {a)}}$; dorsometacarpalis [of digit IV] (Haines, 1939; ; Diogo and Ziermann, 2014; Diogo et al., 2018)

This study. Sus-métacarpo-phalangettien du digitule (Dugès, 1834ª); sus-métacarpophalango-phalangettien du digitule (Dugès, 1834 ${ }^{\mathrm{a}}$ ); interosseus dorsalis [of digit V] (Ecker, 1864); extensor brevis profundus digiti V (Gaupp, 1895' ${ }^{\text {, }}$ 1896; Kändler, 1924:

Dorsometacarpalis proximalis part.a; Ribbing, 1907 , 1911 b; Davies and Burton, 1982; Burton, 1983: part. ${ }^{\text {a }}$; Duellman and digiti $\mathrm{V}$ Trueb, 1986 ; Burton, 1996, 1998a, 1998c; Fabrezi and Langone, 2000: part., ab; Manzano et al., 2007 b; Salgar et al., 2009; Hoyos and Salgar, 2016; Araujo-Vieira et al., 2019); extensor brevis superficialis digiti V? (Kändler, 1924); dorsometacarpalis [of digit V] (Haines, 1939; Diogo and Ziermann, 2014; Diogo et al., 2018); extensor brevis distalis digiti V (Salgar et al., 2009ª)

Dorsometacarpales distales Part of extensores breves profundi (before Burton, 1996) and extensores breves distales (after)

group

This study. Extensor brevis distalis digiti II (Burton, 1998c; Faivovich, 2002: part. a Man-

Dorsometacarpalis indicis distalis zano et al., 2007b; Hoyos and Salgar, 2016; Araujo-Vieira et al., 2019); extensor brevis profundus [of digit II] (Fabrezi and Langone, 2000: part. ${ }^{\text {) }}$ )

Dorsometacarpalis distalis digiti III

This study. Extensor brevis distalis digiti III (Burton, 1996, 1998c; Faivovich, 2002: part. ${ }^{\text {; }}$ Manzano et al., 2007 ); extensor brevis profundus digiti III (Fabrezi and Langone, 2000: part. ${ }^{\mathrm{a}, \mathrm{b}}$; Hoyos and Salgar, 2016: part. ${ }^{\mathrm{a}}$ )

Dorsometacarpalis distalis digiti IV

This study. Extensor brevis profundus digiti IV (Hanna and Barnes, 1991?a,b; Fabrezi and Langone, 2000: part. ${ }^{\mathrm{a}, \mathrm{b}}$; Hoyos and Salgar, 2016: part. $^{\mathrm{a}}$ ); extensor brevis distalis digiti IV (Burton, 1996, 1998c; Manzano et al., 2007'; Araujo-Vieira et al., 2019)

Dorsometacarpalis distalis digiti $\mathrm{V}$

This study. Extensor brevis distalis digiti V (Burton, 1996, 1998c; Manzano et al., 2007º; Hoyos and Salgar, 2016); extensor brevis profundus digiti V (Kändler, 1924a; Fabrezi and Langone, 2000: part.a,b)

VENTRAL LEG

Flexor accessorius

Same (Ribbing, 1909: part., 1911: part.; Diogo and Molnar, 2014; Diogo and Ziermann, 2014; Blotto et al., 2017; Diogo et al., 2018); tarso-sous-phalangettien des trois premiers doigts (Dugès 1834: part. ${ }^{\text {)}}$; transversus plantae (Ecker, 1864; Gaupp, 1895, 1896; Dunlap, 1960; Nussbaum, 1982); tarso-fléchisseur des doigts (Perrin, 1892)

Same (Blotto et al., 2017); tarso-sous-phalangettien des trois premiers doigts (Dugès 1834: part.); transversus plantae anterior (Ecker, 1864); tarso-fléchisseur des doigts (Per-

Flexor accessorius proximalis rin, 1892: part. ${ }^{a}$ ); transversus plantae proximalis (Gaupp, 1895, 1896; Dunlap, 1960; Duellman and Trueb, 1986; Burton, 2001, 2004); transversus [proximal] (Davies and Burton, 1982); transversus plantae proximus (Burton, 1983); flexor accessorius (Diogo and Ziermann, 2014: part.; Diogo et al., 2018: part.) 
APPENDIX 3 continued

\begin{tabular}{ll}
\hline Terminology used in this study & \multicolumn{1}{c}{ Synonyms } \\
\hline & Same (Blotto et al., 2017); tarso-sous-phalangettien des trois premiers doigts (Dugès, 1834: \\
& part.); transversus plantae posterior (Ecker, 1864); tarso-fléchisseur des doigts (Perrin, \\
& 1892: part. a); transversus plantae distalis (Gaupp, 1895, 1896; Dunlap, 1960; Burton, 1983; \\
& Duellman and Trueb, 1986; Burton, 2001, 2004; Faivovich et al., 2005; Salgar et al., 2009; \\
& Hoyos et al., 2014; Araujo-Vieira et al., 2019); transversus [distal] (Davies and Burton, 1982); \\
& flexor accessorius (Diogo and Ziermann, 2014: part.; Diogo et al., 2018: part.) \\
\hline & Same (Diogo and Molnar, 2014; Diogo and Ziermann, 2014; Dos Santos et al., 2017; Diogo \\
& et al., 2018); tibio-sous-tarsien (Dugès, 1834: part. a); plantaris (Ecker, 1864); fléchisseur \\
& interne des doigts (Perrin, 1892: part. a); plantaris profundus (Gaupp, 1895, 1896; Dunlap, \\
& 1960; Dunlap, 1966; Davies and Burton, 1982; Nussbaum, 1982; Burton, 1983; Duellman \\
Tibialis posterior & and Trueb, 1986; Burton, 2001, 2004); flexor accessorius (Ribbing, 1909: part., 1911: part.)
\end{tabular}
and Trueb, 1986; Burton, 2001, 2004); flexor accessorius (Ribbing, 1909: part., 1911: part.)

Same (Diogo and Molnar, 2014; Diogo and Ziermann, 2014; Dos Santos et al., 2017; Diogo et al., 2018); tibio-sous-astragalien (Dugès, 1834); extensor tarsi (Ecker, 1864); rotateur Contrahentium caput longum direct du pied (Perrin, 1892; part, ${ }^{2}$ ); tarsalis posticus (Gaupp, 1896; Dunlap, 1960; Dunlap, 1966; Davies and Burton, 1982; Nussbaum, 1982; Burton, 1983; Duellman and Trueb, 1986; Burton, 2001, 2004; Salgar et al., 2009); pronator profundus (Ribbing, 1909: part., 1911: part.)

Same (Diogo and Molnar, 2014; Diogo and Ziermann, 2014; Diogo et al., 2018); calcanéoscaphoïdien (Dugès, 1834); adductor longus digiti I (Ecker, 1864); rotateur direct du

Interosseus cruris

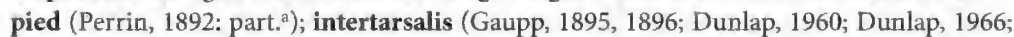
Davies and Burton, 1982; Burton, 1983; Duellman and Trueb, 1986; Burton, 2001, 2004); pronator profundus (Ribbing, 1909: part., 1911: part.)

\begin{tabular}{l}
\hline VENTRAL FOOT \\
\hline Superficial cutaneous tendons \\
\hline Tendines superficiales group \\
Tendo superficialis praehallucis
\end{tabular}

Same (Burton, 2004 ${ }^{\mathrm{a}}$ )

No significant change

Same (Gaupp, 1895' ; Dunlap, 1960ª ; Burton, 2004); tendon fléchisseur de la phalangette de Tergot (Perrin, 1892); flexor primordialis communis (Ribbing, 1909: part.); superficial flexor tendon of prehallux (Dunlap, 1960)

Same (Gaupp, 1895', 1896; Dunlap, 1960; Davies and Burton, 1982; Burton, 1983, 2001, 2004; Faivovich et al., 2005; Salgar et al., 2009; Hoyos et al., 2014; Hoyos and Salgar, 2016;

Tendo superficialis hallucis Blotto et al., 2017); tarso-sous-phalangettien des trois premiers doigts (Dugès 1834: part, $\left.{ }^{a}\right)$; flexor digiti I longus (Ecker, 1864); tendon fléchisseur de la première phalangette (Perrin, 1992); flexor primordialis communis (Ribbing, 1909: part., 1911: part.); flexor tendon of digit I (Dos Santos et al., 2017; Fratani et al., 2018 ${ }^{\mathrm{b}}$ )

Same (Gaupp, 1895', 1896 ; Davies and Burton, 1982 ; Dunlap, 1960; Burton, 2001 ${ }^{\text {b }}, 2004^{\text {b }}$; Faivovich et al., 2005 ; Salgar et al., 2009 ; Hoyos et al., 2014; Hoyos and Salgar, 2016 $6^{\mathrm{b}}$; Blotto et al,, 2017); tarso-sous-phalangettien des trois premiers doigts (Dugès, 1834:

Tendo superficialis digiti II part. ${ }^{a}$ ); flexor digiti II longus (Ecker, 1864); tendon fléchisseur de la deuxième phalangette (Perrin, 1892); flexor primordialis communis (Ribbing, 1909; part., 1911: part.); superficial flexor tendon of digit II (Dunlap, 1960); flexor tendon of digit II (Dos Santos et al., 2017; Fratani et al., 2018

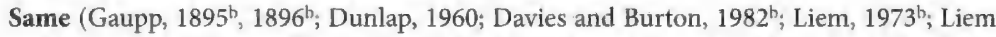
and Hosmer, 1973 ${ }^{\text {b; }}$ Burton, 1983 ${ }^{\text {b }}, 2001^{\text {b }}, 2004^{\text {b; }}$ Faivovich et al., 2005; Salgar et al., 2009 Hoyos et al., 2014; Hoyos and Salgar, 2016 b; Blotto et al., 2017); péronéo-sousphalangettien des trois derniers doigts + tarso-sous-phalangettien des trois premiers doigts (Dugès, 1834: part. a); flexor digitorum III, IV, V longus (Ecker, 1864: part.); tendon fléchisseur de la troisième phalangette (Perrin, 1892); flexor primordialis communis (Ribbing, 1909: part., 1911: part.); superficial flexor tendon of digit III (Dunlap, 1960; Diogo and Ziermann 2014); flexor tendon of digit III (Dos Santos et al., 2017; Fratani et al., 2018 $)$ 
APPENDIX 3 continued

\begin{tabular}{|c|c|}
\hline Terminology used in this study & Synonyms \\
\hline Tendo superficialis digiti IV & 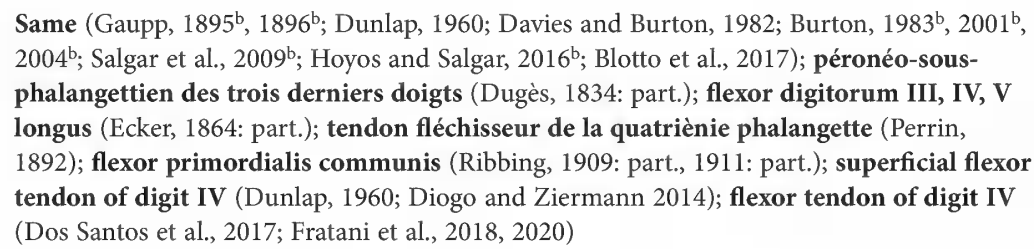 \\
\hline
\end{tabular}
Same (Gaupp, 1895' 1896 b; Dunlap, 1960; Davies and Burton, 1982; Burton, 1983 ${ }^{\mathrm{b}}, 2001^{\mathrm{b}}$, 2004 ; Salgar et al., 2009b; Blotto et al., 2017); péronéo-sous-phalangettien des trois derniers

Tendo superficialis digiti $\mathrm{V}$ doigts (Dugès, 1834: part.); flexor digitorum III, IV, V longus (Ecker, 1864: part.); tendon fléchisseur de la cinquième phalangette (Perrin, 1892); flexor primordialis communis (Ribbing, 1909: part., 1911: part.); superficial flexor tendon of digit V (Dunlap, 1960; Diogo and Ziermann 2014); flexor tendon of digit V (Dos Santos et al., 2017; Fratani et al., 2018 ${ }^{\text {b) }}$

\begin{tabular}{ll}
\hline Lumbricales longi group & No significant change \\
\hline Lumbricalis longus digiti II & $\begin{array}{l}\text { Same (Liem, 1973? ?a,b; Liem and Hosmer, 1973?a, ; ; Burton, 2001); flexor brevis superficialis } \\
\text { [of digit II] (Ribbing, 1911: part.a ); lumbricalis brevis digiti II (Dunlap, 1960: part. }{ }^{\mathrm{a}} \text { ) }\end{array}$ \\
\hline
\end{tabular}

Same (Gaupp, 1895, 1896; Dunlap, 1960; Davies and Burton, 1982; Burton, 1983, 2001, 2004; Faivovich et al., 2005; Salgar et al., 2009; Diogo and Molnar, 2014 ; Diogo and Zier-

Lumbricalis longus digiti III mann, 2014 ; Hoyos and Salgar, 2016; Blotto et al., 2017; Diogo et al., 2018 ${ }^{\text {b }}$; tendini-sousphalanginien du médius (Dugès, 1834); lumbricalis digiti III (Ecker, 1864: part. ${ }^{a}$ ); fléchisseur de la troisième phalangine (Perrin, 1892); flexor brevis superficialis [of digit III] (Ribbing, 1909: part., 1911: part. ${ }^{\text {) }}$

Same (Gaupp, 1895, 1896; Dunlap, 1960; Davies and Burton, 1982; Burton, 1983, 2001, 2004; Faivovich et al., 2005; Salgar et al., 2009; Diogo and Molnar, 2014; Diogo and Zier-

Lumbricalis longus digiti IV mann, 2014 ; Hoyos and Salgar, 2016; Blotto et al., 2017; Diogo et al., 2018 b); tendini-sousphalanginien du quatrième doigt (Dugès, 1834); lumbricalis digiti IV (Ecker, 1864: part. ${ }^{a}$ ); fléchisseur de la quatrième phalanginette (Perrin, 1892); flexor brevis superficialis [of digit IV] (Ribbing, 1909: part., 1911: part.?a)

Same (Gaupp, 1895', 1896; Dunlap, 1960; Davies and Burton, 1982; Burton, 1983, 2001, 2004; Faivovich et al., 2005; Salgar et al., 2009; Diogo and Molnar, 2014 b; Diogo and Zier-

Lumbricalis longissimus digiti mann, 2014 ; Hoyos et al., 2014; Blotto et al., 2017; Diogo et al., 2018 ); tendini-sous-phaIV langinettiens du quatrième doigt (Dugès, 1834: part.?a); lumbricalis digiti IV (Ecker, 1864: part. ${ }^{\mathrm{a}}$ ); fléchisseur de la quatrième phalangine (Perrin, 1892); flexor brevis superficialis [of digiti IV] (Ribbing, 1909: part., 1911: part. ${ }^{a}$ )

Same (Gaupp, 1895', 1896; Dunlap, 1960; Davies and Burton, 1982; Burton, 1983, 2001, 2004; Faivovich et al., 2005; Salgar et al., 2009; Diogo and Molnar, 2014

Lumbricalis longus digiti $\mathrm{V}$ mann, 2014 ; Blotto et al., 2017; Diogo et al., 2018 ); tendini-sous-phalanginiens du digitule (Dugès, 1834); lumbricalis digiti V (Ecker, 1864); fléchisseur de la cinquième phalangine (Perrin, 1892); flexor brevis superficialis [of digit V] (Ribbing, 1909: part., 1911: part.a )

Lumbricales breves group No change

Same (Gaupp, 1895', 1896; Dunlap, 1960; Davies and Burton, 1982; Burton, 1983, 2001, 2004; Faivovich et al., 2005; Salgar et al., 2009; Diogo and Ziermann, 2014 ; Hoyos et al., Lumbricalis brevis hallucis 2014; Blotto et al., 2017; Diogo et al., 2018 ${ }^{\mathrm{b}}$ ); tendini-sous-phalangien du pouce (Dugès, 1834); lumbricalis digiti I (Ecker, 1864); fléchisseur de la première phalange (Perrin, 1892); flexor brevis superficialis [of digit I] (Ribbing, 1909: part., 1911?a)

Same (Gaupp, 1895', 1896; Dunlap, 1960; Davies and Burton, 1982; Burton, 1983, 2001, 2004; Salgar et al., 2009; Diogo and Molnar, 2014 ; Diogo and Ziermann, 2014 ; Hoyos et

Lumbricalis brevis digiti II al., 2014; Blotto et al., 2017; Diogo et al., 2018 b); tendini-sous-phalangien du deuxième doigt (Dugès, 1834); lumbricalis digiti II (Ecker, 1864); fléchisseur de la deuxième phalange (Perrin, 1892); flexor brevis superficialis [of digit II] (Ribbing, 1909: part., 1911: part. $\left.^{a}\right)$; lumbricalis longus digiti II (Liem, 1973a,b) 
APPENDIX 3 continued

\begin{tabular}{|c|c|}
\hline Terminology used in this study & Synonyms \\
\hline Lumbricalis brevis digiti III & 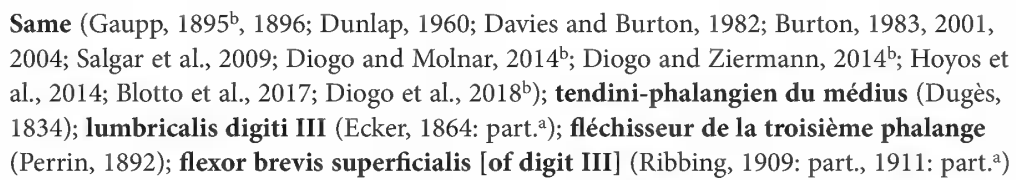 \\
\hline
\end{tabular}

(Perrin, 1892); flexor brevis superficialis [of digit III] (Ribbing, 1909: part., 1911: part.

Same (Gaupp, 1895, 1896; Dunlap, 1960; Davies and Burton, 1982; Burton, 1983, 1986, 2001ª 2004; Salgar et al., 2009; Diogo and Molnar, 2014 ; Diogo and Ziermann, 2014; Hoyos et al., 2014; Blotto et al., 2017; Diogo et al., 2018 ${ }^{\text {b); }}$ tendini-phalangien du Lumbricalis brevis digiti IV quatrième doigt (Dugès, 1834 ${ }^{\mathrm{a}}$ ); tendini-sous-phalanginien du quatrième doigt (Dugès, 1834a); flexor brevis digiti IV (Ecker, 1864a); lumbricalis digiti IV (Ecker, 1864a); fléchisseur de la quatrième phalange (Perrin, 1892); flexor brevis superficialis [of digit IV] (Ribbing, 1909: part., 1911: part. $\left.{ }^{a}\right)$

Same (Gaupp, 1895 , 1896; Dunlap, 1960; Davies and Burton, 1982; Burton, 1983, 1986, 2001, 2004; Faivovich et al., 2005; Salgar et al., 2009; Diogo and Molnar, 2014 ; Diogo and Ziermann, 2014 ; Hoyos et al., 2014; Hoyos and Salgar, 2016; Blotto et al., 2017; Diogo et Lumbricalis brevis digiti V al., 2018 ${ }^{\text {b; }}$ Araujo-Vieira et al., 2019); sous-tarso-ex-phalangien du digitule (Dugès, 1834 a); sous-tarso-in-phalangien du digitule (Dugès, 1834 ); adductor digiti V (Ecker, 1864 ${ }^{\mathrm{a}}$ ); flexor brevis digiti V (Ecker, 1864 $4^{\mathrm{a}}$ ); fléchisseur de la cinquième phalange (Perrin, 1892); flexor brevis superficialis [of digit V] (Ribbing, 1909: part., 1911: part. ${ }^{a}$ )

Same (Ecker, 1864; Gaupp, 1896; Dunlap, 1960; Liem, 1973; Liem and Hosmer, 1973; Nussbaum, 1982; Burton, 1983; Duellman and Trueb, 1986; Burton, 2001, 2004; Faivovich et al., 2005; Salgar et al., 2009; Diogo and Ziermann, 2014; Hoyos et al., 2014; Hoyos and Salgar,

Aponeurosis plantaris 2016; Blotto et al., 2017; Dos Santos et al., 2017; Diogo et al., 2018; Fratani et al., 2018); flexor primordialis communis (Ribbing, 1909: part., 1911: part.); plantar aponeurosis (Davies and Burton, 1982; Burton, 1983, 1986; Diogo and Molnar, 2014; Diogo and Ziermann, 2014; Diogo et al., 2018)

Same (Diogo and Molnar, 2014; Diogo and Ziermann 2014; Blotto et al., 2017; Dos Santos et al., 2017; Diogo et al., 2018; Fratani et al., 2018); péronéo-sous-phalangettien des trois derniers doigts (Dugès, 1834: part. ${ }^{a}$ ); flexor digitorum III, IV, V longus (Ecker, 1864: part.); fléchisseur externe des doigts (Perrin, 1892: part.a); flexor digitorum brevis superficialis

Flexores breves superficiales (Gaupp, 1895, 1896; Dunlap, 1960; Dunlap, 1966; Liem, 1973; Burton, 1983; Duellman and Trueb, 1986; Burton, 2001, 2004; Faivovich et al., 2005; Hoyos and Salgar, 2016; Fabrezi et al., 2017; Araujo-Vieira et al., 2019); flexor accessorius (Ribbing, 1909: part., 1911: part.); flexor digitorum communis (Davies and Burton, 1982); flexor digitorum communis longus (Davies and Burton, 1982); flexor brevis superficialis (Duellman and Trueb, 1986; Burton, 2001); flexor digitorum (Burton, 2001); flexor digiti brevis superficialis (Hoyos et al., 2014)

Contrahentes pedis group Contrahentes

Same (Diogo and Molnar, 2014 ${ }^{\text {b }}$ Diogo and Ziermann, 2014 al., 2018 ); sous-métatarso-phalangien du pouce (Dugès, 1834); flexor brevis digiti I

Contrahentis pedis hallucis (Ecker, 1864); fléchisseur profond de la première phalange (Perrin, 1892); flexor teres hallucis (Gaupp, 1895b 1896; Davies and Burton, 1982; Burton, 2001); contrahentis hallucis (Ribbing, 1909 ${ }^{\mathrm{b}}, 1911^{\mathrm{b}}$; Dunlap, 1960: part. ${ }^{\text {a,b; }}$ B Burton, 2004; Faivovich et al., 2005; Hoyos and Salgar, 2016); opponens hallucis (Salgar et al., 2009?a)

Same (Diogo and Molnar, 2014 ${ }^{\mathrm{b}}$; Diogo and Ziermann, 2014 ; Diogo et al., 2018 );

Contrahentis pedis digiti II fléchisseur profond de la deuxième phalange (Perrin, 1892); contrahentis digiti II (Ribbing, 1909b 1911b; Dunlap, 1960; Salgar et al., 2009; Hoyos and Salgar, 2016)

Same (Diogo and Molnar, 2014 ${ }^{\mathrm{b}}$; Diogo and Ziermann, 2014 ${ }^{\mathrm{b}}$; Diogo et al., 2018 );

Contrahentis pedis digiti III fléchisseur profond de la troisième phalange (Perrin, 1892); contrahentis digiti III (Rib-

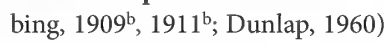

Contrahentis pedis digiti IV Same (Diogo and Molnar, 2014 $4^{\mathrm{b}}$; Diogo and Ziermann, 2014 ${ }^{\mathrm{b}}$; Diogo et al., 2018 ); contrahentis digiti IV (Dunlap, 1960) 
APPENDIX 3 continued

\begin{tabular}{|c|c|}
\hline Terminology used in this study & Synonyms \\
\hline Contrahentis pedis digiti $\mathrm{V}$ & 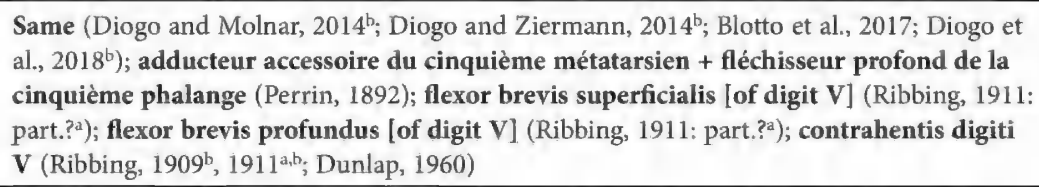 \\
\hline Abductor praehallucis & $\begin{array}{l}\text { Same (Gaupp, 1895, 1896; Dunlap, 1960; Liem, 1973; Davies and Burton, 1982a; Nussbaum, } \\
\text { 1982; Burton, 1983, 2001, 2004; Salgar et al., 2009; Diogo and Molnar, 2014; Diogo and Zier- } \\
\text { mann, 2014; Hoyos et al., 2014; Hoyos and Salgar, 2016; Blotto et al., 2017; Diogo et al., } \\
\text { 2018); tibio-sous-tarsien (Dugès, 1834: part.a); abductor hallucis (Ecker, 1864); adducteur } \\
\text { de la phalangette de l'ergot (Perrin, 1892); adducteur du métatarsien de l'ergot (Perrin, } \\
\text { 1892); flexor brevis superficialis (Ribbing, 1909: part.); abductor praehallucis dorsalis } \\
\text { (Liem and Hosmer, 1973); abductor praehallucis ventralis (Liem and Hosmer, 1973) }\end{array}$ \\
\hline Adductor praehallucis & Same (Burton, 2001; Blotto et al., 2017); abductor brevis plantaris hallucis (Dunlap, 1960: part.") \\
\hline $\begin{array}{l}\text { Abductor brevis plantaris hal- } \\
\text { lucis }\end{array}$ & $\begin{array}{l}\text { Same (Gaupp, 1895', 1896; Dunlap, 1960: part.d; Liem, 1973; Liem and Hosmer, 1973; Davies } \\
\text { and Burton, 1982; Burton, 1983, 2001, 2004; Faivovich et al., 2005; Salgar et al., 2009; Diogo and } \\
\text { Molnar, 2014; Hoyos et al., 2014; Hoyos and Salgar, 2016; Blotto et al., 2017; Diogo et al., 2018); } \\
\text { tibio-sous-tarsien (Dugès, 1834: part. } \text { ); abductor longus digiti I (Ecker, 1864); adducteur } \\
\text { postérieur du premier métatarsien (Perrin, 1892); flexor brevis superficialis (Ribbing, 1909: } \\
\text { part., 1911: part.); abductor plantaris hallucis (Burton, 2001, 2004); flexor accessories } \\
\text { (Hayashi et al., 2015) }\end{array}$ \\
\hline Flexor hallucis accessorius & $\begin{array}{l}\text { Same (Diogo and Ziermann, 2014; Blotto et al., 2017); sous-tarso-métatarso-phalangien } \\
\text { du pouce (Dugès, 1834); opponens hallucis (Ecker, 1864; Gaupp, 1895 }, 1896 \text {; Dunlap, } \\
\text { 1960; Davies and Burton, 1982; Burton, 1983, 1986, 2001, 2004; Salgar et al., 2009?ä; Diogo } \\
\text { and Molnar, 2014; Diogo et al., 2018); fléchisseur primitif du premier métatarsien (Per- } \\
\text { rin, 1892); flexor brevis profundus [of digit I] (Ribbing, 1909, 1911) }\end{array}$ \\
\hline Flexores breves profundi group & Flexores ossum metatarsorum \\
\hline $\begin{array}{l}\text { Flexor hallucis brevis profun- } \\
\text { dus }\end{array}$ & This study \\
\hline
\end{tabular}

Same (Ribbing, 1909, $1911^{\mathrm{b}}$; Diogo and Molnar, 2014 ; Diogo and Ziermann, 2014 ${ }^{\mathrm{b}}$; Blotto et al., 2017; Diogo et al., 2018 ${ }^{\text {b }}$; sous-tarso-métatarsien du deuxième doigt (Dugès, 1834);

Flexor brevis profundus digiti métatarso-métatarsien [of digit II] (Dugès, 1834?"); flexor metatarsi digiti II (Ecker, 1864); déducteur du deuxième doigt (Perrin, 1892); flexor ossis metatarsi digiti II (Gaupp, 1895', 1896; Dunlap, 1960; Davies and Burton, 1982; Burton, 1983, 2001, 2004; Faivovich et al., 2005; Salgar et al., 2009; Hoyos et al., 2014; Hoyos and Salgar, 2016; Araujo-Vieira et al., 2019)

Same (Ribbing, 1909, $1911^{\mathrm{b}}$; Diogo and Molnar, 2014 ; Diogo and Ziermann, 2014 ${ }^{\mathrm{b}}$; Blotto et al., 2017; Diogo et al., 2018 ); Sous-calcanéo-métatarsien du médius (Dugès, 1834);

Flexor brevis profundus digiti

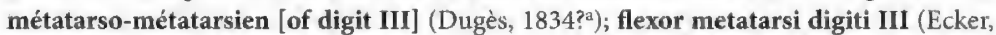
1864); déducteur du troisième doigt (Perrin, 1892); flexor ossis metatarsi digiti III (Gaupp, 1895'b 1896; Dunlap, 1960; Davies and Burton, 1982; Burton, 1983, 2001, 2004; Faivovich et al., 2005; Salgar et al., 2009; Hoyos and Salgar, 2016)

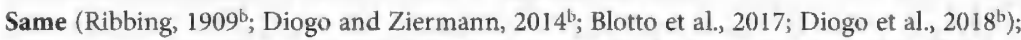
Flexor brevis profundus digiti métatarso-métatarsien [of digit IV] (Dugès, 1834: part.?"); flexor metatarsi digiti IV (Ecker, 1864); déducteur du quatrième doigt (Perrin, 1892); flexor ossis metatarsi digiti IV (Gaupp, 1895', 1896; Dunlap, 1960; Davies and Burton, 1982; Burton, 1983, 2001, 2004; Faivovich et al., 2005; Salgar et al., 2009; Hoyos and Salgar, 2016; Araujo-Vieira et al., 2019) 
APPENDIX 3 continued

\begin{tabular}{ll}
\hline Terminology used in this study & \multicolumn{1}{c}{ Synonyms } \\
\hline $\begin{array}{l}\text { Flexores digitorum minimi } \\
\text { group }\end{array}$ & Flexores teretes, with exception of digit I \\
\hline
\end{tabular}

Flexor minimus hallucis

contrahentis digiti I (Dunlap, 1960: part."); flexor teres hallucis (Burton, 1983, 2004; lucis (Salgar et al., 2009?a); flexor digiti hallucis (Blotto et al., 2017)

Same (Ribbing, 1909 ; Diogo and Ziermann, 2014 ; Blotto et al., 2017; Diogo et al., $2018^{a, b}$ ); sous-métatarso-phalangien du second doigt (Dugès, 1834); flexor digiti II pro-

Flexor minimus digiti II prius (Ecker, 1864); fléchisseur primitif de la deuxième phalange (Perrin, 1892); flexor teres digiti II (Gaupp, 1895 , 1896; Dunlap, 1960; Davies and Burton, 1982; Burton, 1983, 2001, 2004; Salgar et al., 2009)

Same (Ribbing, 1909 ; Diogo and Molnar, 2014 a,b; Diogo and Ziermann, 2014 al, 2017; Diogo et al, 2018 hal ); sous-métatarso-phalangien du médius (Dugès, 1834);

Flexor minimus digiti III flexor digiti III proprius (Ecker, 1864); fléchisseur primitif de la troisième phalange (Perrin, 1892); flexor teres digiti III (Gaupp, 1895', 1896; Dunlap, 1960; Davies and Burton, 1982; Burton, 1983, 2001, 2004; Salgar et al., 2009)

Same (Ribbing, 1909, $1911^{\mathrm{a}, \mathrm{b}}$; Diogo and Molnar, 2014 $4^{\mathrm{a}, \mathrm{b}}$; Diogo and Ziermann, 2014 Blotto et al., 2017; Diogo et al., 2018 $8^{\mathrm{a}, \mathrm{b}}$ ); sous-métatarso-phalangien du quatrième doigt

Flexor minimus digiti IV (Dugès, 1834); flexor digiti IV proprius (Ecker, 1864); fléchisseur primitif de la quatrième phalange (Perrin, 1892); flexor teres digiti IV (Gaupp, 1895'b 1896; Dunlap, 1960; Davies and Burton, 1982; Burton, 1983, 2001, 2004; Salgar et al,, 2009)

Same (Ribbing, 1909 ; Diogo and Ziermann, 2014 ; Blotto et al., 2017; Diogo et al., 2018 $\left.8^{\mathrm{a}, \mathrm{b}}\right)$; sous-métatarso-phalangien du digitule (Dugès, 1834); flexor digiti V proprius

Flexor minimus digiti V (Ecker, 1864); fléchisseur primitif de la cinquième phalange (Perrin, 1892); flexor teres digiti V (Gaupp, 1895', 1896; Dunlap, 1960; Davies and Burton, 1982; Burton, 1983, 2001, 2004; Salgar et al., 2009)

Intermetatarsales group Transversi metatarsorum

Same (Diogo and Molnar, 2014b; Diogo and Ziermann, 2014 ; Blotto et al., 2017; Diogo et al., 2018 b); premier intermétatarsien (Dugès, 1834); interosseus (Ecker, 1864a; Ribbing,

Intermetatarsalis I 1909, 1911); transversus metatarsus I (Ecker, 1864 ab ; Gaupp, 1895 ${ }^{\mathrm{b}}, 1896^{\mathrm{b}}$; Dunlap, $1960^{\mathrm{b}}$; Davies and Burton, 1982; Burton, 1983, 2001, 2004; Salgar et al., 2009); premier intermétatarsien (Perrin, 1892); opponens hallucis (Salgar et al., 2009?a)

Same (Diogo and Molnar, 2014" Diogo and Ziermann, 2014'b; Blotto et al., 2017; Diogo et

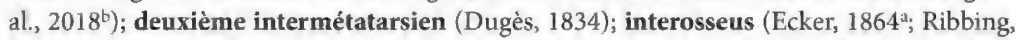

Intermetatarsalis II 1909, 1911); transversus metatarsus II (Ecker, 1864 ${ }^{\text {a, }}$; Gaupp, 1895, $1896^{\text {b; }}$ Dunlap, $1960^{\text {b; }}$; Davies and Burton, 1982; Burton, 1983, 2001, 2004; Faivovich et al,, 2005; Salgar et al., 2009; Hoyos and Salgar, 2016); deuxième intermétatarsien (Perrin, 1892); transversus (Burton, 2001)

Same (Diogo and Molnar, 2014 ; Diogo and Ziermann, 2014 ${ }^{\text {b }}$; Blotto et al., 2017; Diogo et al., 2018 ); troisième intermétatarsien (Dugès, 1834: part. ${ }^{3}$ ); interosseus (Ecker, 1864:

Intermetatarsalis III part. ${ }^{\text {; }}$ Ribbing, 1909, 1911); transversus metatarsus III (Ecker, 1864: part.a,b; Gaupp, $1895^{\mathrm{b}}$, 1896 $\mathrm{b}$; Dunlap, 1960 ${ }^{\mathrm{b}}$; Davies and Burton, 1982; Burton, 1983, 2001, 2004; Faivovich et al., 2005; Salgar et al., 2009; Hoyos and Salgar, 2016); troisième intermétatarsien (Perrin, 1892)

Same (Diogo and Molnar, 2014 ${ }^{\text {b }}$, Diogo and Ziermann, 2014 al., 2018 ); troisième intermétatarsien (Dugès, 1834: part. ${ }^{a}$ ); interosseus (Ecker, 1864: Intermetatarsalis IV part. ${ }^{a}$; Ribbing, 1909, 1911: part. ${ }^{a}$ ); transversus metatarsi III (Ecker, 1864: part. ${ }^{\mathrm{a} . \mathrm{b}}$ ); quatrième intermétatarsien (Perrin, 1892); transversus metatarsus IV (Gaupp, 1895, 1896 ; Dunlap, 1960 b ; Davies and Burton, 1982; Burton, 1983, 2001, 2004; Faivovich et al., 2005; Hoyos and Salgar, 2016; Araujo-Vieira et al., 2019)

Interphalangei group Interphalangeales


APPENDIX 3 continued

\begin{tabular}{ll}
\hline Terminology used in this study & \multicolumn{1}{c}{ Synonyms } \\
\hline Interphalangeus digiti II & This study \\
\hline & Same (Ribbing, 1909 ${ }^{\mathrm{b}}, 1911^{\mathrm{b}}$; Diogo and Molnar, 2014; Diogo and Ziermann, 2014 ${ }^{\mathrm{b}}$; Blotto \\
& et al., 2017; Hayashi et al., 2015 ; Diogo et al., 2018); phalango-phalanginien du médius \\
& (Dugès, 1834); flexor phalangum proprius digiti III (Ecker, 1864); fléchisseur primitif de \\
& la troisième phalangine (Perrin, 1892); interphalangealis digiti III (Gaupp, 1895, $1896 ;$ \\
& Dunlap, 1960; Davies and Burton, 1982; Burton, 1983, 2001, 2004; Salgar et al., 2009) \\
\hline
\end{tabular}

Phalango-phalanginien du quatrième doigt (Dugès, 1834); flexor phalangum proprius

Interphalangeus proximalis digiti IV

dig. IV anterior (Ecker, 1864); fléchisseur primitif de la quatrième phalanginette (Perrin, 1892); interphalangealis proximalis digiti IV (Gaupp, 1895 , 1896; Dunlap, 1960; Davies and Burton, 1982; Burton, 1983, 2001, 2004; Salgar et al., 2009); interphalangeus digit IV (Ribbing, 1909: part. ${ }^{b}, 1911^{\mathrm{a}, \mathrm{b}}$; Diogo and Molnar, 2014: part.; Diogo and Ziermann, 2014: part.b; Hayashi et al., 2015 ; Diogo et al., 2018: part.)

Phalangino-phalanginettien du quatrième doigt (Dugès, 1834); flexor phalangum proprius digiti IV posterior (Ecker, 1864); fléchisseur primitif de la quatrième phalangine

Interphalangeus distalis digiti IV (Perrin, 1892); interphalangealis distalis digiti IV (Gaupp, 1895' ${ }^{\mathrm{b}}$, 1896; Dunlap, 1960; Davies and Burton, 1982; Burton, 2001, 2004; Salgar et al., 2009); interphalangeus digit IV (Ribbing, 1909: part. ${ }^{\text {b; }}$ Diogo and Molnar, 2014: part.; Diogo and Ziermann, 2014: part. ${ }^{\text {b. }}$ Hayashi et al., 2015'; Diogo et al., 2018: part.)

This study. Phalango-phalanginien du digitule (Dugès, 1834); flexor phalangum proprius (Ecker, 1864); fléchisseur primitif de la cinquième phalangine (Perrin, 1892); inter-

Interphalangeus proximalis digiti V phalangealis digiti V (Gaupp, 1895', 1896; Dunlap, 1960; Davies and Burton, 1982; Burton, 1983, 2001, 2004; Salgar et al., 2009); interphalangeus digit V (Ribbing, 1909, $1911^{\text {b; }}$; Diogo and Molnar, 2014; Diogo and Ziermann, 2014 ; Diogo et al., 2018)

Interphalangeus distalis digiti This study DORSAL LEG/FOOT

Same (Gaupp, 1895; Liem, 1970; Diogo and Molnar, 2014; Diogo and Ziermann, 2014; Blotto et al., 2017; Dos Santos et al., 2017; Diogo et al., 2018); péronéo-sus-phalangien du quatrième doigt (Dugès, 1834); extensor longus digiti IV (Ecker, 1864; Gaupp, 1896; Davies and Burton, 1982; Burton, 1983; Duellman and Trueb, 1986; Burton, 2001);

Extensor digitorum longus extenseur commun des doigts (Perrin, 1892); extensor brevis superficialis (Ribbing, 1909: part., 1911: part.); extensor digitorum communis longus (Gaupp, 1895; Dunlap, 1960, 1966; Liem, 1970, 1973; Inger, 1972; Liem and Hosmer, 1973; Burton, 2004; Faivovich et al., 2005; Hoyos et al., 2014; Hoyos and Salgar, 2016; Araujo-Vieira et al., 2019); extensor communis longus (Liem, 1970)

Same (Diogo and Molnar, 2014; Diogo and Ziermann, 2014; Dos Santos et al., 2017; Diogo et al., 2018); calcanéo-sus-métatarsien du digitule (Dugès, 1834); déducteur du cinquième métatarsien (Perrin, 1892); abductor brevis dorsalis digiti V (Gaupp,

Abductor digiti minimi 1895, 1896; Dunlap, 1960; Dunlap, 1966; Davies and Burton, 1982; Burton, 1983; Duellman and Trueb, 1986; Burton, 2001, 2004; Faivovich et al., 2005; Salgar et al., 2009; Hoyos and Salgar, 2016); extensor brevis superficialis (Ribbing, 1909: part., 1911: part.)

Same (Gaupp, 1896; Dunlap, 1960, 1966; Liem, 1970; Davies and Burton, 1982; Burton, 1983; Duellman and Trueb, 1986; Burton, 2001, 2004; Faivovich et al., 2005; Salgar et al., 2009; Diogo and Molnar, 2014; Diogo and Ziermann, 2014; Hoyos and Salgar, 2016; Dos

Tarsalis anticus Santos et al., 2017; Diogo et al., 2018); péronéo-sus-astragalien (Dugès, 1834); flexor tarsi posterior (Ecker, 1864); rotateur inverse du pied (Perrin, 1892: part. ${ }^{\mathrm{a}}$ ); extensor brevis superficialis (Ribbing, 1909: part., 1911: part.)

Extensores breves superficiales group
No change with exception of digit IV 
APPENDIX 3 continued

\begin{tabular}{|c|c|}
\hline Terminology used in this study & Synonyms \\
\hline $\begin{array}{l}\text { Exrensor brevis superficialis } \\
\text { hallucis }\end{array}$ & 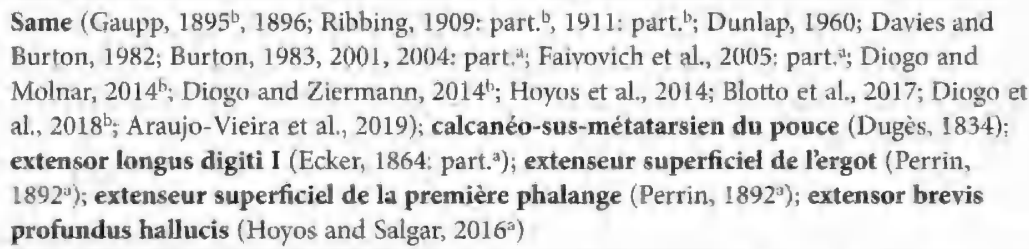 \\
\hline
\end{tabular}

Same (Gaupp, 1895 h, 1896; Ribbing, 1909: part. ${ }^{\text {b }}$ 1911: part. "h; Dunlap, 1960; Davies and

Exiensor brevis superficialis Burton, 1982; Burton, 1983, 2001, 2004: part.".; Faivovich et al., 2005: part.at; Salgar et al., digiti II 2009; Diogo and Molnar, 2014 ${ }^{\mathrm{b}}$; Diogo and Ziermann, 2014 ${ }^{\mathrm{b}}$; Hoyos et al,, 2014; Hoyos and Salgat, 2016; Blotto et al., 2017; Diogo et al., 2018 ); astragalo-sus-phalangien du deuxième doigt (Dugès, 1834); extensor digiti II longus (Ecker, 1864); extenseur superficiel de la deuxième phalange (Perrin, 1892)

Same (Gaupp, 1895, 1896; Ribbing, 1909: part. ${ }^{\text {, }}$ 1911: part."; Dunlap, 1960; Davies and Burton, 1982; Burton, 1983, 2001, 2004; Faivovich et al, 2005; Diogo and Molnar, 2014,

Extensor brevis superficialis digiti III Diogo and Ziermann, 2014 ; Hoyos et al., 2014; Hoyos and Salgar, 2016; Blotto et al., 2017; Diogo et al, 2018 ; Araujo-Vicira et al., 2019); sus-astragalo-phalanginien du médius (Dugès, 1834); astragalo-sus-phalangien dn médius (Dugès, 1834? ); extensor digiti III longus (Ecker, 1864); extenseur superficiel de la troisième phalange (Perrin, 1892)

Same (Ribbing, 1909: part. ${ }^{b}, 1911$ : part. a,b; Dunlap, 1960; Liem and Hosmer, 1973; Burton, 1983?a; Burton, 2004; Faivovich et al., 2005; Diogo and Molnar, 2014 mann, 2014 $4^{\text {b; }}$ Hoyos et al., 2014; Hoyos and Salgar, 2016; Blotto et al., 2017; Diogo et al.,

Extensor brevis superficialis digiti IV $2018^{b}$ ); calcanéo-sus-phalangien du q̨uatrième doigt (Dugès, 1834 $)$; sus-calcanéophalanginien du quatrième doigt (Dugès, 1834ª); extensortes digiti IV breves (Ecker, 1864); extenseur de la quatrième phalange (Perrin, 1892 $)$; extenseur de la quatrième phalanginette (Perrin, 1892 $2^{\mathrm{a}}$ ); extensor brevis medius digiti IV (Gaupp, 1895 ${ }^{\mathrm{A}}$, 1896 Davies and Burton, 1982a; Burton, 1983a, 2001 ${ }^{\mathrm{a}}$; Diogo and Ziermann, 2014?ab)

Same (Gaupp, 1895', 1896; Ribbing, 1909: part.", 1911: part.".; Dunlap, 1960; Liem, $1973^{\text {b; }}$

Extensor brevis superficialis Liem and Hosmer, 1973 ${ }^{\text {h }}$; Burton, 1983, 2001: part.a 2004; Hoyos et al., 2014; Blotto et al., digiti $\mathrm{V}$ 2017; Diogo et al., 2018 ); calcanéo-sus-phalangien du digitule (Dugés, 1834); extensor digiti $\mathrm{V}$ brevis (Ecker, 1864); extenseur de la cinquième phalange (Perrin, 1892)

Extensores breves medii group No change with the exception of digit IV Same (Gaupp, 1896; Dunlap, 1960; Liem, 1973 ${ }^{\text {b; }}$ Liem and Hosmer, 1973 ${ }^{\text {; }}$; Davies and Burton, 1982; Burton, 1983, 2001, 2004: part. ${ }^{\text {; }}$ Faivovich et al., 2005: part. ${ }^{2}$, Salgar et al., 2009;

Extensor brevis medius hallucis Diogo and Molnar, 2014 ${ }^{\text {h}}$; Diogo and Ziermann, 2014?a, ; Hoyos et al., 2014; Blotto et al., 2017; Diogo et al,, 2018 $8^{\text {) }}$; extensor longus digiti I (Ecker, 1864: part. a); extenseur de la première phalange (Perrin, 1892); extensor brevis superficialis [of digit 1] (Ribbing, 1909: part., 1911: part.)

Same (Gaupp, 1896; Dunlap, 1960; Liem, 1973 $3^{\text {b }}$ Liem and Hosmer, 1973 $3^{\text {b }}$ Davies and Burton, 1982; Burton, 1983, 2001, 2004: part."; Faivovich et al., 2005: part."; Salgar et al., 2009; Diogo

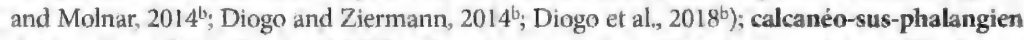

Exiensor brevis medius digiti II du deuxième doigt (Dugès, 1834?"'); astragalo-sus-phalangettien du second doigt (Dugès, 1834?a); extensor digiti 11 brevis (Ecker, 1864); extenseur de la deuxième phalange (Perrin, 1892); extensor brevis superficialis [of digit II] (Ribbing, 1909: part., 1911: part.)

Same (Gaupp, 1896; Dunlap, 1960; Liem, 1973 ${ }^{\text {; }}$ Liem and Hosmer, 1973b; Davies and Bur. ton, 1982; Burton, 1983, 2001, 2004; Faivovich et al., 2005; Diogo and Molnar, 2014

Extensor brevis medius digiti and Ziermann, 2014 ; Hoyos and Salgar, 2016; Diogo et al., 2018 ); astragalo-sus-phaIII langien du médius (Dugès, 1834?:); extensor digiti III brevis (Ecker, 1864); extenseur de la troisième phalange (Perrin, 1892); extensor brevis superficialis [of digit III] (Ribbing, 1909: part., 1911: part.) 
APPENDIX 3 continued

\begin{tabular}{ll}
\hline Terminology used in this study & \multicolumn{1}{c}{ Synonyms } \\
\hline $\begin{array}{ll}\text { Extensor brevis medius digiti } \\
\text { IV }\end{array}$ & $\begin{array}{l}\text { Same (Dunlap, 1960; Diogo and Ziermann, 2014?a, ; Diogo et al., 2018? }{ }^{\text {a,b }} \text { ); extensor brevis } \\
\text { superficialis [of digit IV] (Ribbing, 1909? })\end{array}$ \\
\hline $\begin{array}{l}\text { Dorsometatarsales proximales } \\
\text { group }\end{array}$ & Extensores breves profundi \\
\hline
\end{tabular}

This study. Deux métatarso-sus-phalangettiens du pouce (Dugès, 1834); interosseus dorsalis II (Ecker, 1864 ${ }^{\mathrm{a}}$ ); extenseur profond du premier doigt (Perrin, 1892); extensor

Dorsometatarsalis hallucis proximalis

Dorsometatarsalis proxinalis digiti II

Dorsometatarsalis proximalis digiti III

Dorsometatarsalis proximalis digiti IV

Dorsometatarsalis proximalis digiti $\mathrm{V}$ brevis profundus hallucis (Gaupp, 1895', 1896; Ribbing, 1909' $1911^{\mathrm{b}}$; Dunlap, 1960: part. $^{\text {a }}$ Davies and Burton, 1982; Burton, 1983; Duellman and Trueb, 1986 ${ }^{\text {b }}$ Fabrezi and Langone, 2000 a,b; Burton, 2001; Faivovich, 2002 ; Burton, 2004; Salgar et al., 2009; Hoyos and Salgar, 2016); extensor brevis dorsalis hallucis (Salgar et al., 2009?"); dorsometatarsalis [of digit I] (Diogo and Molnar, 2014; Diogo and Ziermann, 2014; Diogo et al., 2018)

This study. Métatarso-sus-phalangien du deuxième doigt (Dugès, 1834a); métatarso-susphalangettien du second doigt (Dugès, 1834 $4^{\mathrm{a}}$; interosseus dorsalis III (Ecker, 1864 ${ }^{\mathrm{a}}$ ); interosseus dorsalis IV (Ecker, 1864 $)$; extenseur profond du deuxième doigt (Perrin, 1892); extensor brevis profundus digiti II (Gaupp, 1895, 1896 ; Ribbing, 1909 ${ }^{\mathrm{b}}, 1911^{\mathrm{b}}$; Dunlap, 1960: part.a; Davies and Burton, 1982; Burton, 1983; Duellman and Trueb, 1986 ; Fabrezi and Langone, 2000: part. ${ }^{\text {a,b; }}$; Burton, 2001, 2004; Salgar et al., 2009; Hoyos and Salgar, 2016); dorsometatarsalis [of digit II] (Diogo and Molnar, 2014; Diogo and Ziermann, 2014; Diogo et al., 2018)

This study. Deux métatarso-sus-phalangettiens du médius (Dugès, 1834); interosseus dorsalis V (Ecker, 1864 ${ }^{\mathrm{a}}$ ); interosseus dorsalis VI (Ecker, 1864 ${ }^{\mathrm{a}}$ ); extenseur profond du troisième doigt (Perrin, 1892); extensor brevis profundus digiti III (Gaupp, 1895'b 1896; Ribbing, 1909', 1911º; Dunlap, 1960: part."; Davies and Burton, 1982; Burton, 1983; Duellman and Trueb, 1986 ${ }^{\mathrm{b}}$; Fabrezi and Langone, 2000: part. ${ }^{\mathrm{a}, \mathrm{b}}$; Burton, 2001, 2004; Salgar et al., 2009; Hoyos and Salgar, 2016); dorsometatarsalis [of digit III] (Diogo and Molnar, 2014; Diogo and Ziermann, 2014; Diogo et al., 2018)

This study. Deux métatarso-sus-phalangettiens du quatrième doigt (Dugès, 1834); interosseus dorsalis VII (Ecker, 1864 a); interosseus dorsalis VIII (Ecker, 1864 ${ }^{\mathrm{a}}$ ); extenseur profond du quatrième doigt (Perrin, 1892); extensor brevis profundus digiti IV (Gaupp, 1895, 1896; Ribbing, 1909 ${ }^{\mathrm{b}}, 1911^{\mathrm{a}, \mathrm{b}}$; Dunlap, 1960: part. ${ }^{\mathrm{a}}$; Davies and Burton, 1982; Burton,

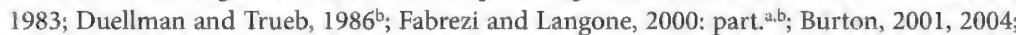
Salgar et al., 2009; Hoyos and Salgar, 2016: part." ); dorsometatarsalis [of digit IV] (Diogo and Molnar, 2014; Diogo and Ziermann, 2014; Diogo et al., 2018)

This study. Deux métatarso-sus-phalangettiens du digitule (Dugès, 1834); interosseus dorsalis IX (Ecker, 1864 ); interosseus dorsalis X (Ecker, 1864"); abductor digiti V brevis (Ecker, 1864"); extenseur profond du cinquième doigt (Perrin, 1892); extensor brevis

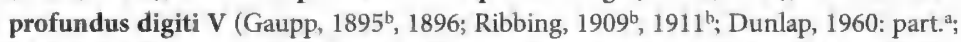
Davies and Burton, 1982; Davies and Burton, 1982; Burton, 1983; Duellman and Trueb, 1986 ; Fabrezi and Langone, 2000: part. ${ }^{\mathrm{a}, \mathrm{b}}$; Burton, 2001, 2004; Faivovich et al., 2005; Salgar et al., 2009; Hoyos and Salgar, 2016); extensor brevis superficialis digiti V (Davies and Burton, 1982a; Burton, 2001: part. ${ }^{a}$; Hoyos and Salgar, 2016 ); dorsometatarsalis [of digit V] (Diogo and Molnar, 2014; Diogo and Ziermann, 2014; Diogo et al., 2018)

\begin{tabular}{|c|c|}
\hline $\begin{array}{l}\text { Dorsometatarsales distales } \\
\text { group }\end{array}$ & $\begin{array}{l}\text { Part of the extensores breves profundi (before Faivovich, 2002) and extensores breves } \\
\text { distales (after) }\end{array}$ \\
\hline $\begin{array}{l}\text { Dorsometatarsalis hallucis } \\
\text { distalis }\end{array}$ & $\begin{array}{l}\text { This study. Extensor brevis profundus hallucis (Dunlap, 1960: part. }{ }^{a} \text { ); extensor brevis } \\
\text { distalis hallucis (Faivovich, 2002 } \text {; Burton, 2004) }\end{array}$ \\
\hline $\begin{array}{l}\text { Dorsometatarsalis distalis digiti } \\
\text { II }\end{array}$ & $\begin{array}{l}\text { This study. Extensor brevis profundus digiti II (Dunlap, 1960: part. }{ }^{\text {;a }} \text { Fabrezi and Lan- } \\
\text { gone, 2000: part., }{ }^{\mathrm{a} b} \text { ); extensor brevis distalis digiti II (Faivovich, 2002; Burton, 2004; } \\
\text { Araujo-Vieira et al., 2019) }\end{array}$ \\
\hline
\end{tabular}


APPENDIX 3 continued

\begin{tabular}{|c|c|}
\hline Terminology used in this study & Synonyms \\
\hline $\begin{array}{l}\text { Dorsometatarsalis distalis digiti } \\
\text { III }\end{array}$ & $\begin{array}{l}\text { This study. Extensor brevis profundus digiti III (Dunlap, 1960: part. }{ }^{\text {a; }} \text { Fabrezi and Lan- } \\
\text { gone, 2000: part.a,b); extensor brevis distalis digiti III (Faivovich, 2002; Burton, 2004) }\end{array}$ \\
\hline $\begin{array}{l}\text { Dorsometatarsalis distalis digiti } \\
\text { IV }\end{array}$ & $\begin{array}{l}\text { This study. Extensor brevis profundus digiti IV (Dunlap, 1960: part. }{ }^{\text {a; }} \text { Fabrezi and Lan- } \\
\text { gone, 2000: part.a,b; Hoyos and Salgar, 2016: part. a); extensor brevis distalis digiti IV } \\
\text { (Faivovich, 2002; Burton, 2004; Araujo-Vieira et al., 2019) }\end{array}$ \\
\hline $\begin{array}{l}\text { Dorsometatarsalis distalis digiti } \\
\text { V }\end{array}$ & $\begin{array}{l}\text { This study. Extensor brevis profundus digiti V (Dunlap, 1960: part.a; Fabrezi and Lan- } \\
\text { gone, 2000: part., } \text { ); extensor brevis distalis digiti V (Faivovich, 2002; Burton, 2004; } \\
\text { Araujo-Vieira et al., 2019) }\end{array}$ \\
\hline $\begin{array}{l}\text { Abductores breves dorsales } \\
\text { group }\end{array}$ & Digit I: no uniform name; digits II-III: first reported here \\
\hline $\begin{array}{l}\text { Abductor brevis dorsalis hal- } \\
\text { lucis }\end{array}$ & 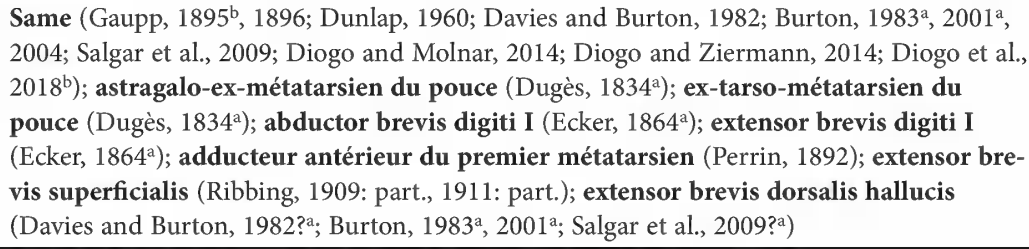 \\
\hline $\begin{array}{l}\text { Abductor brevis dorsalis digiti } \\
\text { II }\end{array}$ & This study \\
\hline $\begin{array}{l}\text { Abductor brevis dorsalis digiti } \\
\text { III }\end{array}$ & This study \\
\hline Abductor proprius digiti IV & $\begin{array}{l}\text { Same (Gaupp, 1895, 1896; Dunlap, 1960; Davies and Burton, 1982; Burton, 1983; Burton, } \\
\text { 2001, 2004; Salgar et al., 2009; Diogo and Molnar, 2014; Diogo and Ziermann, 2014; Diogo } \\
\text { et al., 2018); métatarso-métatarsien (Dugès, 1834: part.?a); quatrième intermétatarsien } \\
\text { accessoire (Perrin, 1892); interosseus [of digit IV] (Ribbing, 1909: part.. 1911: part. }{ }^{\text {a }} \text { ) }\end{array}$ \\
\hline
\end{tabular}

\section{Comments on Some Synonyms}

We discuss the names included in the synonym list that require clarifications or justifications and are arranged in the following sections: (1) palmar surface and the forearm muscles; (2) dorsal surface of the hand and the forearm muscles; (3) plantar surface; and (4) dorsal surface of the foot. The comments for each muscle are headed by the name of the muscle, as listed in the synonym list. Finally (section 5), we make comments and define names for some novel muscles and tendons that are not addressed in Results and Discussion.

Palmar Surface of the Hand

\section{Dugès (1834)}

M. EPITROCHLO-SOUS-PHALANGETTIEN: The portions that correspond to our tendines superfi- ciales digitorum III-V are the tendons depicted with number 118 in Dugès (1834: fig. 51) on digits III-V, respectively. The tendon of digit II of that figure corresponds to the tendon of insertion of our $\mathrm{m}$. flexor indicis superficialis proprius.

M. SOUS-CARPO-PHALANGETTIEN DE L'INDEX: Probably corresponds to the fleshy portion of our $\mathrm{m}$. flexor indicis superficialis proprius.

M. SOUS-CARPO-MÉTACARPO-PHALANGIEN DU DIGITULE: Might correspond to our lateral $\mathrm{m}$. lumbricalis brevis digiti V; the figures and descriptions are not detailed enough to unequivocally separate it from our $\mathrm{m}$. abductor digiti minimi.

M. TENDINI-PHALANGIEN DU DIGITUlE: Corresponds to our medial $\mathrm{m}$. lumbricalis brevis digiti $\mathrm{V}$.

M. SOUS-CARPO-PHALANGIEN DU MÉDIUS: The identity of the medial muscle, depicted as " 107 " in Dugès (1834: fig. 51), is unclear (while the lateral 
muscle, depicted as "106," corresponds to our m. flexor brevis profundus digiti III). It might correspond to (1) our $\mathrm{m}$. flexor minimus digiti III; however, the origin shown in his figure 51 seems to be from a distal carpal, while our $\mathrm{m}$. flexor minimus digiti III originates from the metacarpal; (2) our m. contrahentis digiti III; or (3) an atypical portion of the $\mathrm{m}$. flexor brevis profundus digiti III. We consider the first option more probable since the $\mathrm{m}$. contrahentis digiti III was not reported in Pelophylax, studied by Dugès (1834; see Ecker, 1864; Gaupp, 1896), nor in the family Ranidae to which it belongs (this study). However, it requires postulating that the origin of the $\mathrm{m}$. flexor minimus is erroneously figured (it should be on metacarpal III, and dorsally to our m. intermetacarpalis I). In line with the problems highlighted here, Gaupp (1896: 163) stated that the description of Dugès (1834) was incorrect.

M. SOUS-CARPO-PHALANGIEN DE L'ANNULAIRE: The muscle depicted as " 111 " in Dugès (1834: fig. 51) corresponds topologically to our m. flexor minimus digiti IV. However, in his figure 51 it seems to originate from the distal carpals, not from metacarpal IV, as it is characteristic of this muscle. Alternatively, it might be the $\mathrm{m}$. contrahentis digiti IV, characterized by an origin on the distal carpals. In such a case, the $\mathrm{m}$. flexor minimus digiti IV was overlooked and not figured by Dugès (1834). The problem here is partially equivalent to the one discussed above for the $\mathrm{m}$. sous-carpo-phalangien du médius, with the difference that the $\mathrm{m}$. contrahentis digiti IV is present in Pelophylax (Gaupp, 1896: his caput volare of the $\mathrm{m}$. flexor teres digiti IV), as well as in other ranids (this study). We consider it more likely that this muscle of Dugès (1834) corresponds to our $\mathrm{m}$. contrahentis digiti IV. The muscle depicted as " 110 " in his figure 51 corresponds to our $\mathrm{m}$. flexor brevis profundus digiti IV, for which it is discarded to be our $\mathrm{m}$. flexor minimus digiti IV.

M. SOUS-PYRO-PRÉ-MÉTACARPIEN DU DIGITULE: This muscle probably corresponds to our $\mathrm{m}$. abductor digiti minimi, although the figures and descriptions are not detailed enough to unequivocally separate it from our lateral $\mathrm{m}$. lumbricalis brevis digiti $\mathrm{V}$.

Ecker (1864)

M. FLEXOR DIGITI III LONGUS S. SUBLIMIS: This muscle includes our tendo superficialis digiti III and our $\mathrm{m}$. caput profundum digiti III. See Discussion for further comments.

M. FLEXOR DIGITI IV LONGUS S. SUBLIMIS: This muscle includes our tendo superficialis digiti IV and our $\mathrm{m}$. lumbricalis longus digiti IV. Gaupp (1896: 163) stated that Ecker (1864) did not describe the $\mathrm{m}$. lumbricalis longus digiti IV. However, it was partially and superficially described, and also figured by Ecker (1864: 105, fig. 78), under the name m. flexor digiti IV longus $\mathrm{s}$, sublimis (see also Gaupp, 1895: 196, who supports our interpretation). See Discussion for further comments.

M. PLEXOR DIGITI V LONGUS S. SUBLIMIS: This muscle includes our tendo superficialis digiti $\mathrm{V}$ and our $\mathrm{m}$. lumbricalis longus digiti V. Gaupp (1896: 165) stated that Ecker (1864) did not describe the $\mathrm{m}$. lumbricalis longus digiti V. However, it was partially and superficially described, and also figured by Ecker (1864: 106, fig. 78), under the name $\mathrm{m}$. flexor digiti V longus s. sublimis (see also Gaupp, 1895: 196, who supports our interpretation). See Discussion for further comments.

MM, FLEXORES DIGITI III BREVES S. PROFUNDI I AND II, AND M. INTEROSSEUS VOLARIS (OF DIGIT III): The $\mathrm{mm}$. flexores digiti III breves $\mathrm{s}$. profundi I and II correspond to our slip of the $\mathrm{m}$. lumbricalis brevis digiti III with origin on the distal carpals (but see below) and to our slip of this muscle with origin on the flexor plate. However, the $\mathrm{m}$. interosseus volaris, described and figured for digit III, cannot be discarded to be part of our $\mathrm{m}$. lumbricalis brevis digiti III with origin on the distal carpals.

Furthermore, with the available figures and descriptions, the $\mathrm{m}$. interosseus volaris of digit III cannot also be discarded to correspond to our $\mathrm{m}$. contrahentis digiti III. The fact that neither Gaupp 
(1896), who studied the same species as Ecker (1864), nor we in our ranoid sample (the only exception is the microhylid Ctenophryne geayi) found an $\mathrm{m}$. contrahentis digiti III turns the interpretation of this $\mathrm{m}$. interosseus as our $\mathrm{m}$. contrahentis digiti III an implausible hypothesis.

MM. FLEXORES DIGITI IV BREVES S. PROFUNDI I AND II: These two muscles correspond to our medial and lateral $\mathrm{m}$. lumbricalis brevis digiti IV.

M. ABDUCTOR PRIMUS DIGITI V AND M. FLEXOR DIGITI V: The latter corresponds to our medial $\mathrm{m}$. lumbricalis brevis digiti $\mathrm{V}$. Concerning the $\mathrm{m}$. abductor primus digiti $\mathrm{V}$, the description of Ecker (1864: 106) reporting an insertion on the phalanx is compatible with its identity as our lateral $\mathrm{m}$. lumbricalis brevis digiti V. However, the morphology and position evidenced in his figures 78-79 are also compatible with our $\mathrm{m}$. abductor digiti minimi. The fact may be that the $\mathrm{m}$. abductor digiti minimi is totally or partially fused with the lateral $\mathrm{m}$. lumbricalis brevis digiti $\mathrm{V}$, as occurs in many species of different clades (this study; see also Gaupp, 1896: 166-167, for a close relationship between both muscles in Pelophylax, which is the same taxon studied by Ecker, 1864). Thus, the $\mathrm{m}$. abductor primus digiti $\mathrm{V}$ of Ecker (1864) might be a combination of our $\mathrm{m}$. abductor digiti minimi and our lateral $\mathrm{m}$. lumbricalis brevis digiti $\mathrm{V}$.

Gaupp $(1895,1896)$

M. FLEXOR TERES DIGITI IV: The portion of this muscle that corresponds to our $\mathrm{m}$. contrahentis digiti IV is his "caput volare," while the portion that corresponds to our $\mathrm{m}$. flexor minimus digiti IV is his "caput dorsale."

Ribbing (1907)

M. FLEXOR PRIMORDIALIS COMMUNIS: This muscle is essentially our $\mathrm{m}$. flexor digitorum communis, and includes our flexor plate and tendines superficiales digitorum II-V, which Ribbing (1907) considers to be the tendons of insertion of this muscle. This is partially in agreement with our study, with the difference that our flexor plate is considered a strong tendon of insertion (follow- ing Abdala and Diogo, 2010) product also from the contribution of the $\mathrm{m}$. flexor accessorius (see further comments about the relationship between these elements in Discussion).

A particular comment is required for the inclusion of our tendo superficialis indicis as part of the $\mathrm{m}$. flexor primordialis communis of Ribbing (1907). Most neobatrachians do not have a tendo superficialis indicis (sensu this study) and have instead an $\mathrm{m}$. flexor indicis superficialis proprius, which inserts by a long palmar flexor tendon on the distal phalanx, as do the tendo superficialis indicis. Differently, the tendo superficialis indicis is present in Discoglossus pictus (one of the species studied by Ribbing, 1907), and we consider the $\mathrm{m}$. flexor primordialis communis a synonym of our tendo superficialis indicis on this basis.

M. FLEXOR BREVIS SUPERFICIALIS (OF PREPOLLEX): Our $\mathrm{m}$. adductor pollicis probably corresponds to the portion of the $\mathrm{mm}$. flexores breves superficiales of Ribbing (1907: 597) that inserts on prepollex.

M. CONTRAHENTIS Digiti IV: Ribbing (1907: 632), in an apparent typing error regarding the digit number of this muscle, wrote "caput volare des M. flexor teres digiti $V$ ” quoting Gaupp (1896), instead of "caput volare des M. flexor teres digiti IV," as Gaupp (1896: 164-165, fig. 94) refers to it. Further support for this putative typing error is the fact that (1) the description given by Ribbing (1907: 632) refers to the muscle of digit IV (and not digit V); and (2) it is correctly mentioned later by Ribbing (1911: 13) as the "caput volare des Flexor teres digiti IV". Abdala and Diogo (2010), Diogo and Abdala (2010), and Diogo et al. (2018) replicated this typing error, probably originated by Ribbing (1907).

MM. FLEXORES MINIMI DIGITORUM III AND IV: The muscles depicted (in error?) as the medial/radial portion of $\mathrm{m}$. flexor brevis profundus of digits III and IV in Ribbing (1907: figs. 13-14) are probably our $\mathrm{mm}$. flexores $\mathrm{min}$ imi digitorum III and IV; see Discussion for further comments. 
M. FLEXOR MINIMUS Digiti V: The muscle depicted as $\mathrm{m}$. flexor brevis profundus in his figure 13 probably corresponds to our $\mathrm{m}$. flexor minimus digiti $\mathrm{V}$, based on position and morphology; see Discussion for further comments.

M. FLEXOR BREVIS PROFUNDUS AND M. FLEXOR BREVIS SUPERFICIALIS (OF DIGIT V): It is not possible to unequivocally identify to which muscles of our terminology they correspond. Ribbing (1907: 596) equals the $\mathrm{m}$. abductor primus digiti V of Gaupp (1896) to his $\mathrm{m}$. flexor brevis superficialis. However, based on morphology and position, the muscle depicted in his figure 14 of Pelophylax as the lateral muscle of his $\mathrm{m}$. flexor brevis profundus resembles more the $\mathrm{m}$. abductor primus digiti $\mathrm{V}$ of Gaupp (which is our $\mathrm{m}$. abductor digiti minimi), than the $\mathrm{m}$. opponens digiti $\mathrm{V}$ of Gaupp (which is our $\mathrm{m}$. flexor brevis profundus digiti V). Following this, we include it with question marks within the synonym list of our $\mathrm{m}$. abductor digiti minimi and our $\mathrm{m}$. flexor brevis profundus digiti $\mathrm{V}$.

M. CAPUT LONGUM MUSCULARUM CONTRAHENTIUM: The proximal and distal portions of this muscle correspond to our $\mathrm{m}$. contrahentis caput longus proximalis and $\mathrm{m}$. contrahentis caput longus distalis, respectively; see Discussion further comments.

Ribbing (1911)

General comments: Some identities interpreted here must be considered with caution since the musculature of Pipa is highly modified, obscuring their identities and correspondences with muscles of other clades. Furthermore, Ribbing (1911) did not present figures supporting his descriptions.

A problematic point is that the species of $P$ ipa studied by Ribbing (1911) is not clearly identified. However, based on a comparison of some peculiar characteristics described by Ribbing (1911) with our data (mainly for the possession of the $\mathrm{m}$. flexor minimus indicis), we conclude that he probably studied Pipa pipa. We employ this putative taxonomic identification (men- tioned as Pipa cf. pipa) as a partial guide for the preparation of the synonym list.

M. FLEXOR PRIMORDIALIS COMMUNIS: Same considerations as above for Ribbing (1907).

M. INTERPHALANGEUS DIGITI IV: Ribbing (1911: 14) considered that there is probably a remnant of this muscle in Pipa cf. pipa, without further comments. This muscle is absent in the single specimen of Pipa pipa studied by us,

M. INTERPHALANGEUS DIGITI V: Ribbing (1911: 14) described a muscle that he tentatively assigned to the $\mathrm{m}$. interphalangeus of digit $\mathrm{V}$. His doubts may have arisen from its atypical morphology, as well as from his comparisons of Pipa cf. pipa with Discoglossus pictus and Pelophylax cf. esculenta (and probably other species listed by Ribbing, 1907, but not clearly stated). Discoglossus (this study) and Pelophylax (Gaupp, 1896: 166, figs. 92-94) have a medial and a lateral slip, with their fleshy origins over the basal phalanx of digit $\mathrm{V}$, and lack an intermediate slip. Distinctly, the $\mathrm{m}$. interphalangeus digiti $\mathrm{V}$ of Pipa pipa is represented only by an intermediate slip. It arises on the distal end of metacarpal $\mathrm{V}$ (thus, proximally in comparison to most species; e.g., Burton, 1996, 1998a; this study), and its origin is on the tendon of insertion of the $\mathrm{m}$. flexor minimus digiti $\mathrm{V}$, as described by Ribbing (1911) and corroborated in this study. Despite both conditions being uncommon for the $\mathrm{m}$. interphalangeus digiti $\mathrm{V}$, we consider it as such for this study.

M. FLEXOR BREVIS PROFUNDUS DIGITI V: It is tentatively considered to be our homonym muscle. We observed this muscle in Pipa pipa, and it corresponds to the $\mathrm{m}$. flexor brevis profundus digiti V figured for Pipa carvalhoi (see muscle number 6 in pl. 9C).

M. ABDUCTOR DIGITI V: See muscle label 7-8 in plate $9 \mathrm{C}$ of Pipa carvalhoi. The same problems regarding the identities of the abductor muscles of digit $\mathrm{V}$ discussed for Pipa carvalhoi applies to our sample of Pipa pipa and P. cf. pipa of Ribbing (1911).

M. CAPUT LONGUM MUSCULARUM CONTRAHENTIUM: Same comments as above for Ribbing (1907). 
M. FLEXOR ACCESSORIUS (LATERALIS AND MEDIALIS): See Discussion.

Kändler (1924)

TENDO SUPERFICIALIS INDICIS, M, FLEXOR INDICIS SUPERFICIALIS PROPRIUS, AND M. LUMBRICALIS BREVIS INDICIS: Kändler (1924: fig. 23a) indicates that in Bombina bombina (as Bombinator igneus) there is a fleshy portion corresponding to the $\mathrm{m}$. flexor indicis superficialis proprius. However, this muscle is absent in our examined specimen (see char. 1). The fleshy portion he refers to probably corresponds to our $\mathrm{m}$. lumbricalis brevis indicis, while the tendinous attachment to the palmar surface of the distal phalanx corresponds to our tendo superficialis indicis.

M. ADDUCTOR POLLICIS: Kändler (1924: fig. 23a) indicated a tendinous portion as corresponding to the $\mathrm{m}$. adductor pollicis in Bornbina bombina. This portion probably corresponds to a tendinous connection between the flexor plate and the prepollex (similar to the tendines superficiales of the digits). The $\mathrm{m}$. adductor pollicis of $B$. bombina is very different to the element labeled as such by Kändler (1924), as exemplified in plate $9 \mathrm{~B}$ by $B$. orientalis.

M. ABDUCTOR SECUNDUS DIGITI V: The muscle labeled as such by Kändler (1924: fig. 21a) for Bufo bufo (as Bufo vulgaris) probably corresponds to our $\mathrm{m}$. abductor brevis digiti $\mathrm{V}$, which is present in the Bufonidae studied by us. The muscle labeled only as "abd" (abbreviation for abductor) in his figure 23a of Hyla arborea clearly corresponds to our $\mathrm{m}$. abductor brevis digiti $\mathrm{V}$.

Liem (1970)

FIRST TENDO SUPERFICIALIS: As inferred from the description of Liem (1970; 14), his "first tendo superficialis" is a combination of our tendo superficialis indicis and our $\mathrm{m}$. flexor indicis superficialis proprius.

Davies and Burton (1982)

General comments: The study of Davies and Burton (1982) is restricted to Rheobatrachus silus (Myobatrachidae). In the following discus- sion, we compare their descriptions with our observations from this species.

M. CAPUT PROFUNDUM TENDONIS SUPERFICIALIS DIGITI III: This name corresponds to our m. caput profundum digiti III. However, the specimen studied by us has an extremely reduced m. caput profundum digiti III, which has a typical origin from the distal carpals (contra Davies and Burton, 1982). The description given by Davies and Burton (1982) for his m. caput profundum tendonis superficialis digiti III fits with our slip of the m. lumbricalis brevis digiti III with origin on the flexor plate.

M. LUMBRICALIS BREVIS INDICIS: The muscle described by Davies and Burton (1982; 512) under this name is probably composed of portions of our (1) $\mathrm{m}$. lumbricalis brevis indicis; (2) medial $\mathrm{m}$. flexor indicis brevis profundus; and (3) $\mathrm{m}$. contrahentis indicis.

The specimen studied by us has a complete fusion between the $\mathrm{m}$. lumbricalis brevis indicis and the medial $\mathrm{m}$. flexor indicis brevis profundus. The portion inserting on the metacarpophalangeal joint is inferred to correspond to the former, while the penniform insertion on metacarpal $\mathrm{II}$ is assignable to the $\mathrm{m}$. flexor indicis brevis profundus. This explains the penniform insertion along the entire lateral surface of metacarpal II described by Davies and Burton (1982) for their $\mathrm{m}$. lumbricalis brevis indicis.

We also observed the $\mathrm{m}$. contrahentis indicis with origin from the distal carpals (which is typical for this muscle) and from the adjoining proximal end of metacarpal III. This might explain the origin from metacarpal III described by Davies and Burton (1982) for their m. lumbricalis brevis indicis.

M. ABDUCTOR PRIMUS DIGITI V AND M. ABDUCTOR SECUNDUS DIGITI V: Davies and Burton (1982: 512) considered the former to be present, but it went undescribed. The $\mathrm{m}$. abductor secundus digiti $V$ was described as arising from the distal carpals (as centrale postaxiale) and inserting on the laterodistal surface of metacarpal $\mathrm{V}$. This origin and insertion is characteristic of our $\mathrm{m}$. abductor digiti minimi instead, while 
the $\mathrm{m}$. abductor secundus digiti $\mathrm{V}$ usually arises from the ulnare (and from the distal end of radioulna in some species) and has a very proximal insertion on the base of metacarpal V (Burton, 1998a; this study). We observed in this species a muscle that we interpreted as a fused $\mathrm{m}$. abductor digiti minimi and $\mathrm{m}$. abductor secundus digiti $\mathrm{V}$, since its origin covers the region of origin of these two muscles.

Finally, we observed an additional abductor muscle inserting on the metacarpophalangeal joint of digit $\mathrm{V}$, similar to the one depicted for Pipa carvalhoi (pl.9C, muscle labeled 7-8). The identity of this muscle is controversial, as commented for $P$. carvalhot in the legend of plate $9 \mathrm{C}$. It is unclear whether the reference of an "abductor primus digiti V" of Davies and Burton (1982) corresponds to this abductor muscle since they did not describe it.

\section{Burton (1983)}

Superficial APONEURosis: It is unclear whether this aponeurosis is part of a differentiated portion of the flexor plate (sensu this study), or if it corresponds to another kind of connective tissue located between the tegument and the flexor plate.

M. LUMBRICALIS BREVIS INDICIS: The characteristics of the muscle labeled by Burton (1983: fig. 6B) under this name fit better with our $m$. contrahentis indicis. This assertion is based on its relatively lateral position over metacarpal n and lateropalmar insertion on the basal phalanx of digit II. However, Burton (1983) stated that the $\mathrm{m}$. flexor teres indicis (a name that usually corresponds to our $\mathrm{m}$. contrahentis indicis) is absent. At this point, it is unclear whether the $\mathrm{m}$. lumbricalis brevis indicis of Burton (1983) corresponds to our homonym muscle (with an atypical lateral position and insertion) or our $\mathrm{m}$. contrahentis indicis.

M. LUMBRICALIS BREVIS DIGITI V: The "medial m. lumbricalis brevis digiti V" of Burton (1983) probably corresponds to our $\mathrm{m}$. flexor brevis profundus digiti $\mathrm{V}_{2}$ based on position and morphological similarity, as evidenced in his figure $6 \mathrm{C}$.
M. OPPONENS DIGITI V: This muscle corresponds to our $\mathrm{m}$. contrahentis digiti $\mathrm{V}$, based on position and morphological similarity, as evidenced in his figure $6 \mathrm{C}$.

M. ABDUCTOR PRIMUS DIGITI $V$ AND $M$. ABDUCTOR SECUNDUS DIGITI V: Following Burton (1983: fig. 6E), the $\mathrm{m}$. abductor primus digiti $\mathrm{V}$ corresponds to our $\mathrm{m}$. abductor secundus digiti $\mathrm{V}$, and his $\mathrm{m}$. abductor secundus digiti $\mathrm{V}$ corresponds to our $\mathrm{m}$. abductor digiti minimi. However, a cross error in the labeling of his figure $6 \mathrm{C}$ may be inferred from the description of Burton (1983: 317). This description suggests that (1) his $\mathrm{m}$. abductor primus digiti $\mathrm{V}$ corresponds to our $\mathrm{m}$. abductor digiti minimi, and (2) his $\mathrm{m}$. abductor secundus digiti $\mathrm{V}$ corresponds to our homonym muscle.

M. ABDUCTOR POLLICIS AND M. ADDUCTOR pollicis: A cross error in the labeling of Burton (1983: fig. 6C) may be inferred from the description (Burton, 1983: 315). Following the figure, the proximal element labeled as "adductor pollicis" corresponds to our $\mathrm{m}$. pronator quadratus, while the distal element labeled as "abductor pollicis" corresponds to our $\mathrm{m}$. adductor pollicis. However, considering his description, his $\mathrm{m}$. abductor pollicis corresponds entirely to our $\mathrm{m}$. pronator quadratus, while the portion of his $\mathrm{m}$. adductor pollicis from the centrale preaxiale corresponds to our homonym muscle. The deeper portion described under the name of $\mathrm{m}$. adductor pollicis (with origin on the ulnare; not figured by him) corresponds to our $\mathrm{m}$. pronator quadratus as well. This muscle usually originates from the distal end of radioulna and the ulnare (e.g., Burton, 1996, 1998a; this study).

M. TRANSVERSUS METACARPI I AND MUSClE "M.": This unnamed muscle " $M$ " corresponds to a distal portion of our $\mathrm{m}$. intermetacarpalis $\mathrm{I}$, in agreement with Burton (1998b).

Burton (1998a, 1998b)

M. LUMBRICALIS BREVIS OF BURTON (1998a: CHAR. 7): In his character 7, Burton (1998a) scored the presence of a muscle described as "slips from the dorsal surface of the tendo super- 
ficialis digiti III to the basal phalanx." In another passage, Burton (1998a: 69) mentioned this muscle as part of the $\mathrm{m}$. lumbricalis brevis digiti III, and commented that this issue would be further studied in a coming contribution (in reference to Burton, 1998b). From the comparison of the taxonomic distributions and descriptions, it is clear that the muscle of character 7 of Burton (1998a) is the m. lumbricalis longus digiti III of Burton (1998b), which corresponds to our m. lumbricalis longus digiti III. Additionally, the $\mathrm{m}$. lumbricalis longus digiti III described for Heleophrynidae (Burton, 1998a: 66) corresponds to our homonym muscle.

M. FLEXOR TERES DIGITI IV: Burton (1998b) reported this muscle as composed of a ventral and dorsal portion/head in several ranoids. However, the ventral portion of all or most of them corresponds to our m. contrahentis digiti IV, while the dorsal portion to our $\mathrm{m}$. flexor minimus digiti IV.

Faivovich (2002)

M. LUMBRiCALIS LONGUS Digiti V: The "lumbricalis longus digiti V" of Faivovich (2002: 373, 375,383 ) is a misspelling for $\mathrm{m}$. "lumbricalis brevis digiti V" (J.F., personal obs.), and corresponds to the $\mathrm{m}$. lumbricalis brevis digiti $\mathrm{V}$ of Faivovich (2002: fig. 13). His m. lumbricalis brevis digiti V corresponds to our homonym muscle.

Manzano et al. (2007)

Tendo superficialis (of digit II): The tendo superficialis of digit II indicated for Pseudis minuta by Manzano et al. (2007: fig. 5C) corresponds to the tendon of insertion of our $\mathrm{m}$. flexor indicis superficialis proprius. According to our observations, our tendo superficialis indicis is absent in P. minuta.

Manzano et al. (2008)

M. LUMBRICALIS LONGUS DIGITI IV: The branches of this muscle reported as inserting on Penultimate Phalanx IV correspond to our $\mathrm{m}$. lumbricalis longus digiti IV, while the branches inserting on the distal extremity of metacarpal
IV correspond to our $\mathrm{m}$. lumbricalis brevis digiti IV. See Discussion for further comments.

Aguilar and Valencia (2009)

M. LUMBRICALIS LONGI (OF DIGIT III): All the references to this muscle probably correspond to our m. lumbricalis brevis digiti III, with the exception of its report in T. macrostermum. In this species it probably corresponds to our $\mathrm{m}$. lumbricalis longus digiti III, as discussed by Barrionuevo (2017).

Salgar et al. (2009)

M. OPPONENS Digiti V: This muscle probably corresponds to our $\mathrm{m}$. flexor brevis profundus digiti V. Its identity requires corroboration because Salgar et al. (2009) reported this muscle to be partially fused with the abductor muscles of digit $\mathrm{V}$.

Abdala and Diogo (2010), and Diogo and Abdala (2010)

General comments: We discuss these two contributions together since similar comments apply to them. A conflict between the identities of (1) the mm. flexores breves profundi and the $\mathrm{mm}$. flexores digitorum minimi, and (2) the $\mathrm{mm}$. flexores breves superficiales and the $\mathrm{mm}$. lumbricales is addressed in Discussion.

M. Contrahentis (of Prepollex): Abdala and Diogo (2010: 12) and Diogo and Abdala (2010: 381) considered that the m. adductor pollicis (sensu this study) is probably part of their $\mathrm{mm}$. contrahentes. Following this, we include this association with a question mark in the synonym list.

M. CONTRAhentis indicis: Abdala and Diogo (2010: 32) and Diogo and Abdala (2010: 381) mentioned the $\mathrm{m}$. contrahentis on digit II, which corresponds to the concept of our homonym muscle. However, Abdala and Diogo (2010: 33) and Diogo and Abdala (2010: 382383 ), in the discussion of the $\mathrm{mm}$. flexores digitorum minimi sensu Ribbing (1907), included within this group the "flexor teres I" of Burton (1998b). This implies an apparent typing error 
for "flexor teres II" or the equivalent "flexor teres indicis" (this last term is the effectively employed by Burton, 1998b). Furthermore, the m. flexor teres indicis of Burton (1998b) corresponds to our $\mathrm{m}$. contrahentis indicis, and not to our $\mathrm{m}$. flexor minimus indicis.

M. CONTRAHENTIS DIGITI IV: They stated that the "caput volare des $\mathrm{m}$. flexor teres digiti $\mathrm{V}$ " of Gaupp (1896) corresponds to their $\mathrm{m}$. contrahentis of digit IV. This is in agreement with our concept and name, although it must be stated that Gaupp's correct name for it is "caput volare des $\mathrm{m}$. flexor teres digiti IV"; see further comments for this muscle above under Ribbing's (1907) section.

M. FLEXOR BREVIS PROFUNDUS (OF DIGIT V): Abdala and Diogo (2010: 33) and Diogo and Abdala (2010: 382) considered that the $\mathrm{m}$. abductor secundus digiti $\mathrm{V}$ is probably part of their mm. flexores breves profundi. Following this, we express this association with question marks in the synonym list.

\section{Diogo and Ziermann (2014)}

GENERAL COMMENTS: The hand terminology of Diogo and Ziermann (2014) is largely based on their table 2, which follows Diogo and Abdala (2010) and Diogo and Tanaka (2012). We consider these two articles, as well as the descriptions of Eleutherodactylus coqui given by Diogo and Ziermann (2014), for the elaboration of the synonym list and discussion of the relevant points. Note that some muscles are absent in this species, but we add them to the synonym list if their concepts are clearly established in the mentioned articles. A conflict between the identities of (1) the $\mathrm{mm}$. flexores breves profundi and the $\mathrm{mm}$. flexores digitorum minimi, and (2) the $\mathrm{mm}$. flexores breves superficiales and the mm. lumbricales is addressed in Discussion.

M. CONTRAHENTIS (OF PREPOLleX): Diogo and Ziermann (2014: 92, table 2) considered the $\mathrm{m}$. adductor pollicis (their concept of this muscle equals ours) probably part of their mm. contrahentes or their m. pronator quadratus. Following this, we denote both associations with question marks in the synonym list. See Discussion for further comments.

Mm. Contrahentes digitorum: Diogo and Ziermann (2014) did not explicitly state to which names from the literature their $\mathrm{mm}$. contrahentes digitorum correspond, and they are not clearly described nor unequivocally figured. There is a single mention in their section of results (p. 92) while describing Eleutherodactylus coqui: "The contrahentes digitorum constitute the second layer of intrinsic hand muscles and run from the carpal region to the proximal region of digits 2 , 3,4 , and 5 ." The only figure with a label showing $\mathrm{mm}$. contrahentes is their figure $2 \mathrm{D}$ for the developmental stage 11, and denotes "Anlage hand muscle (mainly seem intermetacarpales and/or contrahentes)." The identity of their mm. contrahentes cannot be established from that figure. Similarly, comments in their discussion are also inconclusive regarding the identity of the involved muscles.

We also studied Eleutherodactylus coqui and, as in most nobleobatrachians, only the $\mathrm{mm}$. contrahentes of digits II and V are present. Despite the lack of an explicit description and figures that unequivocally define the concept of the $\mathrm{mm}$. contrahentes of Diogo and Ziermann (2014), we include these muscles in the synonym list because their identity is clearly stated in the explicit reference to Diogo and Abdala (2010). We denote the probable absence of the $\mathrm{mm}$. contrahentes of digits III and IV in E. coqui with question marks in the synonym list.

M. FLEXOR INDICIS BREVIS PROFUNDUS: Diogo and Ziermann (2014: 92-93, table 2) refer to the $\mathrm{m}$. opponens pollicis sensu Ecker (1889) and Duellman and Trueb (1986), and consider it as being part of their mm. flexores breves profundi. The use of "pollicis" is probably a lapsus for "indicis," since Ecker (1889) and Duellman and Trueb (1986) employed the terms $m$. opponens digiti II and $\mathrm{m}$. opponens indicis, respectively. Additionally, Gaupp (1896), whose terminology is generally followed by Duellman and Trueb (1986: 350 ), refers to this muscle as the $\mathrm{m}$. opponens indicis. 
M. ABDUCtor Digiti Minimi: Diogo and Ziermann (2014: table 2) considered the $\mathrm{m}$. abductor secundus digiti $\mathrm{V}$ (their concept of this muscle equals ours) as probably part of the $\mathrm{m}$. abductor digiti minimi. We include this association with question marks in the synonym list.

Barrionuevo (2017)

M. LUMBRICALIS BREVIS DIGITI III: It corresponds to our homonym muscle, which typically inserts on the metacarpophalangeal joint and/or basal phalanx. It was described as inserting on the "distal phalanx" and on "basal phalanx" in different parts of the paragraph where it is discussed by Barrionuevo (2017: 48). Nevertheless, the mention of "distal phalanx" is a lapsus for "basal phalanx" (J.S. Barrionuevo, personal commun.), confirming its identity as m. lumbricalis brevis.

Diogo et al. (2018)

General COMments: A conflict between the identities of (1) the $\mathrm{mm}$. flexores breves profundi and the $\mathrm{mm}$. flexores digitorum minimi, and (2) the $\mathrm{mm}$. flexores breves superficiales and the $\mathrm{mm}$. lumbricales is addressed in Discussion.

M. Contrahentis digiti III: Diogo et al. (2018: 454) stated that there are apparently four $\mathrm{mm}$. contrahentes attaching to digits II-V (one for each digit) in their sample of Lithobates pipiens. The presence of the $\mathrm{m}$. contrahentis in digit III is extremely uncommon within Neobatrachia (this study). The slip of digit III mentioned by Diogo et al. (2018) probably corresponds to our $\mathrm{m}$. lumbricalis brevis originating from the carpals, which co-occurs with the slip from the flexor plate in several ranoids (including our sample of Lithobates; see chars. 5-6 and appendices 4-5).

M. Contrahentis Digiti IV: See comments for this muscle above in Ribbing's (1907) section.

M. FLEXOR BREVIS PROFUNDUS DIGITI V: Their $\mathrm{m}$. abductor secundus digiti $\mathrm{V}$ (which concept equals ours) is considered as being probably part of their mm. flexores breves profundi (2018:
455). Denoting this possibility, we include it in the synonym list of the $\mathrm{m}$. abductor secundus digiti $\mathrm{V}$ with question marks.

M. Contrahentis (of Prepollex): Diogo et al. (2014: 454) considered their m. adductor pollicis (which concept equals ours) to be probably part of their mm. contrahentes digitorum. Following this, we denote this association with question marks in the synonym list of the $\mathrm{m}$. adductor pollicis.

Fratani et al. (2018)

M. FLEXOR INDICIS SUPERFICIALIS PROPRIUS: The flexor tendon described by Fratani et al. (2018) is the tendon of insertion of our m. flexor indicis superficialis proprius, and it is not homologous with our tendo superficialis indicis.

Garg and Biju (2019)

M. LUMBRICALIS BREVIS DIGITI III: The muscle labeled as " 1 " by Garg and Biju (2019) is probably part of our $\mathrm{m}$. lumbricalis brevis digiti III.

M. LUMBRICALIS BREVIS DIGITI IV: The muscle labeled as "2" by Garg and Biju (2019) is probably part of our m. lumbricalis brevis digiti IV.

Dorsal Surface of the Hand

Dugès (1834)

M. SUS-MÉTACARPO-PHALANGO-PHALANGETTIEN DE L'INDEX: This muscle corresponds to our lateral $\mathrm{m}$. dorsometacarpalis indicis proximalis; the medial $\mathrm{m}$. dorsometacarpalis indicis proximalis was not described by Dugès (1834).

M. SUS-MÉTACARPO-PHALANGO-PHALANGETTIEN DU MÉDIUS AND M. SUS-MÉTACARPO-PHALANGETTIEN DU MÉDIUS: Both muscles probably correspond to our medial $\mathrm{m}$. dorsometacarpalis proximalis digiti III. However, its correspondence to part of our m. extensor brevis medius digiti III cannot be discarded.

M. SUS-MÉTACARPO-PHALANGETTIEN DE L'ANnulaire: Corresponds to our medial $\mathrm{m}$. dorsometacarpalis proximalis digiti IV. 
M. SUS-MÉTACARPO-PHALANGO-PHALANGETTIEN DE L'ANNULAIRE: Corresponds to our lateral $\mathrm{m}$. dorsometacarpalis proximalis digiti IV.

M. SUS-MÉTACARPO-PHALANGETTIEN DU DIGITULE: Corresponds to our medial m. dorsometacarpalis proximalis digiti $\mathrm{V}$.

M. SUS-MÉTACARPO-PHALANGO-PHALANGETTIEN DU DIGITULE: Corresponds to our lateral $\mathrm{m}$. dorsometacarpalis proximalis digiti $\mathrm{V}$.

Ecker (1864)

M. EXTENSOR DIGITI II PROPRIUS LONGUS: The portion of this muscle with origin on metacarpal II corresponds to our $\mathrm{m}$. dorsometacarpalis proximalis digiti II. Additionally, this muscle is described as having two heads, one that corresponds to our $\mathrm{m}$. extensor indicis brevis superficialis, and a second one originating from the distal end of radioulna. This second head is only tentatively assigned to our m. extensor indicis brevis superficialis (see description of Triprion petasatus for further comments regarding this head from the radioulna as the "extensor brevis-like" muscle).

M. EXTENSOR DIGITI II PROPRIUS BREVIS: The portion of this muscle with origin on metacarpal II corresponds to our $\mathrm{m}$. dorsometacarpalis proximalis digiti II.

M. EXTENSOR DIGITI 11 II PROPRIUS: The portion of this muscle with origin on metacarpal III corresponds to our $\mathrm{m}$. dorsometacarpalis proximalis digiti III.

M. EXTENSOR DIGITI IV PROPRIUS: The portion of this muscle with origin on metacarpal IV corresponds to our $\mathrm{m}$. dorsometacarpalis proximalis digiti IV.

M. INTEROSSEUS DORSALIS (OF DIGIT IV): The portion of this muscle with origin on the carpal elements probably corresponds to our m. extensor brevis medius digiti IV.

Gaupp $(1895,1896)$

M. EXTENSOR INDICIS BREVIS SUPERFICIALIS: As Ecker (1864), Gaupp (1895, 1896) described a head from the distal end of radioulna that probably corresponds to a portion of our hom- onym muscle; the comments above for Ecker (1864) apply here as well.

Ribbing (1907)

M. EXTENSOR Digitorum COMMUNis: This muscle corresponds to our $\mathrm{m}$. extensor digitorum and to the head of our $\mathrm{m}$. abductor pollicis longus with origin on the humerus.

M. ABDUCTOR DIGITI II: The portion of this muscle with origin on the radioulna corresponds to our $\mathrm{m}$. abductor pollicis longus, while the portion with origin on the carpals corresponds to our $\mathrm{m}$. abductor indicis brevis dorsalis, as evidenced by the description of Ribbing $(1907 ; 667)$.

MM. EXTENSORES BREVES SUPERFICIALES (OF DIGITS II-IV): The superficial muscles of this group described by Ribbing (1907: 668) correspond to our $\mathrm{mm}$. extensores breves superficiales digitorum II-V, while the deep muscles to our $\mathrm{mm}$. extensores breves medii digitorum II-IV.

Ribbing (1911)

M. EXTENSOR DIGITORUM COMMUNIS: Same comments as above for Ribbing (1907).

MM. EXTENSORES BREVES SUPERFICIALES (OF DIGITS II-IV): Almost the same comments as above for Ribbing (1907). The exception is the presence of the $\mathrm{m}$. extensor indicis brevis medius. This muscle is apparently absent in the species of Pipa examined by us ( $P$ ipa pipa was not studied for the dorsal surface of the hand). This fact suggests that the identity of $\mathrm{mm}$. extensores breves of digit II referred by Ribbing (1911: 17) requires confirmation.

Kändler (1924)

M. ABDUCTOR INDICIS LONGUS: Kändler (1924: 212, fig. 23) described two heads of his m. abductor indicis longus for Hyla arborea, a "Caput inferius" and a "Caput breve." The former corresponds to our $\mathrm{m}$. abductor pollicis longus, while his "Caput breve" corresponds to our $\mathrm{m}$. extensor indicis brevis superficialis that, as it is common in Hylidae (this study), inserts partially on metacarpal II.

Similarly, Kändler (1924: 213) described two heads of his $\mathrm{m}$. abductor indicis longus in Alytes obstetricans, one from the radioulna and 
other from the radiale. The former corresponds to our $\mathrm{m}$. abductor pollicis longus, while the head from the radiale corresponds to a portion of our $\mathrm{mm}$. extensores breves of digit II (probably part of our $\mathrm{m}$. extensor indicis brevis superficialis).

M. EXTENSOR INDICIS BREVIS SUPERFICIALIS AND M. EXTENSOR INDICIS BREVIS MEDIUS: Kändler (1924: 200-201) mentioned a portion of the $\mathrm{m}$. extensor indicis brevis superficialis with origin on the element Y (as Centrale) in Rana temporaria. This portion probably corresponds to our $\mathrm{m}$. extensor indicis brevis medius.

M. EXTENSOR BREVIS SUPERFICIALIS DIGITI V: Based on position and fibers orientation, the muscle labeled as such by Kändler (1924: fig. 23a) for Hyla arborea probably corresponds to a portion of our medial $\mathrm{m}$. dorsometacarpalis proximalis digiti $\mathrm{V}$.

M. EXTENSOR BREVIS PROFUNDUS DIGITI V: Based on position and attachments, the portion of the muscle labeled as such by Kändler (1924: fig. 23a) for Hyla arborea corresponds to our medial $\mathrm{m}$. dorsometacarpalis distalis digiti V.

Haines (1939)

General comments: The number system employed for the hand digits is I-IV.

Liem (1970)

General comments: The number system employed for the hand digits is I-IV.

M. EXTENSOR INDICIS BREVIS SUPERFICIALIS: Our m. extensor indicis brevis superficialis is mentioned by Liem (1970) as "externus digitorum brevis superficialis" (in his fig. 9), as "extensor brevis superficialis of the 1st digit" (in table 5), and as "extensor digitorum superficialis of the first finger."

Burton (1983)

EXTENSOR BREVIS PROFUNDUS DIGITI IV: The accessory head described by Burton (1983) with origin on the centrale postaxiale is probably part of our $\mathrm{m}$. extensor brevis superficialis digiti IV.
EXTENSOR BREVIS PROFUNDUS DIGITI V: The accessory head described by Burton (1983) with origin on the centrale postaxiale is probably part of our $\mathrm{m}$. extensor brevis superficialis digiti $\mathrm{V}$.

ABDUCTOR INDICIS BREVIS DORSALIS: Also mentioned as $\mathrm{m}$. abductor indicis brevis.

Duellman and Trueb (1986)

M. EXTENSOR INDICIS BREVIS SUPERFICIALIS: Also mentioned as m. extensor indicis brevis.

Hanna and Barnes (1991)

M. EXTENSOR BREVIS PROFUNDUS DIGITI IV: Hanna and Barnes (1991: fig. 9) referred to the "third digit" in Osteopilus septentrionalis; we interpret it as corresponding to our digit IV. Since the distinction between the mm. extensores breves distales and extensores breves profundi (our mm. dorsometacarpales distales and proximalis, respectively) was subsequently done by Burton (1996), we try below to establish the identity of their muscles for digit IV.

The medial $\mathrm{m}$. dorsometacarpalis distalis digiti IV is absent in the Osteopilus septentrionalis examined by us, for which the medial tendon of insertion of their figure 9 might correspond to our medial $\mathrm{m}$. dorsometacarpalis proximalis digiti IV. The correspondence of the lateral tendon of insertion of that figure is unclear, since this species has two tendons attaching to the distal interphalangeal joint/distal phalanx, one corresponding to our lateral $\mathrm{m}$. dorsometacarpalis proximalis digiti IV and the other to our lateral $\mathrm{m}$. dorsometacarpalis distalis digiti IV.

\section{Burton (1998a)}

M, EXTENSOR INDICIS BREVIS SUPERFICIALIS: As Ecker (1864) and Gaupp (1896), Burton (1998a) described a head from the distal end of radioulna under this name. It probably corresponds to part of our homonym muscle.

Fabrezi and Langone (2000)

General comments: Fabrezi and Langone (2000) studied the mm. extensores breves pro- 
fundi (generically corresponding to our $\mathrm{mm}$. dorsometacarpales proximales) in Allophryne ruthveni. They described these muscles as having elongated fibers, and illustrated them in their figure 7 . They also stated that the $\mathrm{mm}$. extensores breves distales are absent (generically corresponding to our mm. dorsometacarpales distales). We studied Allophryne ruthveni and found the presence of $\mathrm{mm}$. dorsometacarpales distales. We discuss below the presence of $\mathrm{mm}$. dorsometacarpales proximales with elongated fibers reaching the phalanx and the presence of $\mathrm{mm}$. dorsometacarpales distales in each digit.

M. EXTENSOR INDICIS BREVIS PROFUNDUS: We observed a medial and lateral $\mathrm{m}$. dorsometacarpalis indicis proximalis with fibers reaching the phalanx (agreeing with Fabrezi and Langone, 2000) and also a medial and lateral $\mathrm{m}$. dorsometacarpalis indicis distalis.

M. EXTENSOR BREVIS PROFUNDUS DIGITI III: We observed a medial and lateral $\mathrm{m}$. dorsometacarpalis proximalis digiti III with fibers reaching the phalanx (agreeing with Fabrezi and Langone, 2000) and a lateral $\mathrm{m}$. dorsometacarpalis distalis digiti III.

M. EXTENSOR BREVIS PROFUNDUS DIGITI IV: We observed a medial m. dorsometacarpalis proximalis digiti IV with fibers reaching the phalanx (agreeing with Fabrezi and Langone, 2000) and a lateral dorsometacarpalis distalis digiti IV.

M. EXTENSOR BREVIS PROFUNDUS DIGITI V: We observed a medial $\mathrm{m}$. dorsometacarpalis proximalis digiti $\mathrm{V}$ with fibers reaching the phalanx (agreeing with Fabrezi and Langone, 2000) and medial and lateral $\mathrm{m}$. dorsometacarpalis distalis digiti $\mathrm{V}$.

Faivovich (2002)

MM. EXTENSORES BREVES DISTALES DIGITORUM II-III: The muscles described under this name by Faivovich (2002) in his state 1 of characters 55 and 57 probably correspond to our lateral $\mathrm{m}$, dorsometacarpalis proximalis of digits II and III, respectively. The muscles described in his state 0 of characters 55 and 57 correspond to our lateral $\mathrm{m}$. dorsometacarpalis distalis of digits II an III, respectively. This conclusion is based on the fact that when the mm. dorsometacarpales proximales have fibers reaching the phalanx (thus, emulating the $\mathrm{mm}$. dorsometacarpales distales), they can be differentiated from the $\mathrm{mm}$. dorsometacarpales distales by the more proximal origin on the metacarpals, while the $\mathrm{mm}$. dorsometacarpales distales have their origins restricted to the distal end of the metacarpals (Burton, 1996, 1998c; this study). Nevertheless, there are cases in which this distinction is not clear, as further addressed in Discussion.

Manzano et al. (2008)

M. ABDUCTOR INDICIS LONGUS: Also as m. adductor indicis longus (a misspelling).

Salgar et al. (2009)

M. EXTENSOR BREVIS DISTALIS DIGITI V: It corresponds to our lateral $\mathrm{m}$. dorsometacarpalis proximalis digiti $\mathrm{V}$, based on the fact that it has a more proximal origin than the $\mathrm{mm}$. dorsometacarpales distales, and the fibers do not reach the basal phalanx, as it is also characteristic of the $\mathrm{mm}$, dorsometacarpales proximales.

Hoyos and Salgar (2016)

M. EXTENSOR BREVIS PROFUNDUS DIGITI III: The muscle over the lateral surface of the basal phalanx of digit III labeled as such in their figure 3 , and one of the three heads they discuss (p. 150) corresponds to our lateral $\mathrm{m}$. dorsometacarpalis distalis digiti III. This muscle is also present, with the same morphology and topology, in the specimen of Dendropsophus luddeckei studied by us. Furthermore, the fibers of the lateral $\mathrm{m}$. dorsometacarpalis proximalis digiti III do not reach the phalanges in our specimen, preventing confusion with the $\mathrm{m}$. dorsometacarpalis distalis digiti III.

M. EXTENSOR BREVIS PROFUNDUS DIGITI IV: The muscle labeled as such in their figure 3 over the lateral surface of the basal phalanx of digit IV corresponds to our lateral $\mathrm{m}$. dorsometacarpalis distalis digiti IV. This muscle is also present, with the same morphology and position, in the specimen of Dendropsophus luddeckei studied by us. Furthermore, the fibers of the lateral $\mathrm{m}$. dorsometacarpalis proximalis digiti IV do not reach the phalanges in 
our specimen, preventing its confusion with the $\mathrm{m}$. dorsometacarpalis distalis digiti IV.

Plantar Surface of the Foot

Dugès (1834)

M. TENDINI-SOUS-PHALANGINETTIENS DU QUATRIÈME DOIGT: This name refers to his muscles number 207 and 208. The latter clearly corresponds to our $\mathrm{m}$. lumbricalis longissimus digiti IV, while the identity of the former is unclear. It might correspond to our $\mathrm{m}$. lumbricalis longissimus digiti IV (as interpreted by Gaupp, 1896: 209), or to an additional atypical tendon of insertion of our $\mathrm{m}$. lumbricalis longus digiti IV (in common with the $\mathrm{m}$. lumbricalis longissimus digiti IV). Additionally, muscle number 208 might be a distal fleshy portion of our $\mathrm{m}$. lumbricalis longissimus digiti IV (sensu Burton, 2001), while the muscle 207 might be the main fleshy portion of our $\mathrm{m}$. lumbricalis longissimus digiti IV.

M. MÉTATARSO-MÉTATARSIEN (OF DIGIT II): This name refers to his muscle number 177 and probably corresponds to the slip or portion of our $\mathrm{m}$. flexor brevis profundus digiti II inserting dorsally to our $\mathrm{m}$. intermetatarsalis II (see also Gaupp, 1896; 206, who argues along the same line). Our interpretation is reinforced by the fact that the $\mathrm{m}$. flexor brevis profundus digiti IV (for which only the dorsal portion to the $\mathrm{m}$. intermetatarsalis is present; see his fig. 55) is also named "métatarso-métatarsien."

M. MÉTATARSO-MÉTATARSIEN (OF DIGIT III): This name refers to his muscle number 176 and probably corresponds to the slip or portion of our $\mathrm{m}$. flexor brevis profundus digiti III inserting dorsally to our $\mathrm{m}$. intermetatarsalis III; the discussion above for "Métatarso-métatarsien (of digit II)" also applies here.

M. MÉTATARSO-MÉTATARSIEN (OF DIGIT IV): This name refers to his muscle number 175 and corresponds to our m. flexor brevis profundus digiti IV. Dugès (1834) probably included our $\mathrm{m}$. abductor proprius digiti IV within this name as both muscles are morphologically and topologi- cally similar, and no specific comments were made for a muscle attributable to our $\mathrm{m}$. abductor proprius digiti IV.

M. TROISIÈME INTERMÉtATARSIEN: See discussion below under the "Mm. interossei" of Ecker (1864), which applies here as well.

M. TIBIO-SOUS-TARSIEN: The portion of this muscle inserting on the prehallux corresponds to our $\mathrm{m}$. abductor praehallucis, while the portion inserting on metatarsal I corresponds to our $\mathrm{m}$. abductor brevis plantaris hallucis. The remaining portion corresponds to our $\mathrm{m}$. tibialis posterior.

M. TENDINI-PHALANGIEN DU QUATRIÈME DOIGT: This muscle corresponds to our medial $\mathrm{m}$. lumbricalis brevis digiti IV.

M. TENDINI-SOUS-PHALANGINIEN DU QUATRIÈME DOIGT: This muscle corresponds to our lateral $\mathrm{m}$. lumbricalis brevis digiti IV.

M. SOUS-TARSO-IN-PHALANGIEN DU DIGITULE: This muscle corresponds to our medial $\mathrm{m}$. lumbricalis brevis digiti $\mathrm{V}$.

M. SOUS-TARSO-EX-PHALANGIEN DU DIGITULE: This muscle corresponds to our lateral $\mathrm{m}$. lumbricalis brevis digiti $\mathrm{V}$.

M. PÉRONÉO-SOUS-PHALANGETTIEN DES TROIS DERNIERS DOIGTS AND M. TARSO-SOUS-PHALANGETTIEN DES TROIS PREMIERS DOIGTS: DugéS (1834) considered our tendines superficiales of digits I and II to be the tendons of insertion of the tarso-sous-phalangettien des trois premiers doigts (our $\mathrm{m}$. flexor accessorius). Similarly, Dugés (1834) considered our tendo superficialis digiti III to be the fusion of the tendons of insertion of his péronéo-sous-phalangettien des trois derniers doigts (our mm. flexores breves superficiales) and his tarso-sous-phalangettien des trois premiers doigts (our $\mathrm{m}$. flexor accessorius).

An alternative treatment (e.g., Burton, 2004; this study) is to consider that the tendines superficiales digitorum I-II arise from the aponeurosis plantaris while the tendo superficialis digiti III originates from the aponeurosis plantaris and/or the $\mathrm{mm}$. flexores breves superficiales (depending on the species). The question that arises here is that our $\mathrm{m}$. flexor accessorius distalis (= the distal portion of the tarso-sous-phalangettien des 
trois premiers doigts of Dugès) usually has a fleshy insertion on the dorsal surface of the aponeurosis plantaris in the region of origin of the tendines superficiales digitorum I-III, without forming any discrete tendon of insertion (which is the case of the species studied by Dugès, 1834). Nevertheless, a few species have the $\mathrm{m}$. flexor accessorius distalis modified into discrete muscular slips that contribute to the formation of the tendo superficialis of digits I and/or II (Burton, 2004: characters 28 and 31; this study).

\section{Ecker (1864)}

M. LUMBRICALIS DIGITI III: The portion of this muscle that corresponds to our $\mathrm{m}$. lumbricalis longus digiti III is the one that attaches to the second phalanx of digit III, depicted as $13^{\prime}$ in the figures of Ecker (1864). The portion that corresponds to our $\mathrm{m}$. lumbricalis brevis digiti III is the slip that attaches to the basal phalanx of digit III, depicted as 13 in his figures.

M. LUMBRICALIS DIGITI IV: This muscle combines our $\mathrm{m}$. lumbricalis longus digiti $\mathrm{IV}, \mathrm{m}$. lumbricalis longissimus digiti $\mathrm{IV}$, and the medial $\mathrm{m}$. lumbricalis brevis digiti IV.

MM. interossei: Ecker (1864) described three muscles under this generic name, but without assigning specific numbers in his figures. However, in his figure 91 , he used the names " $\mathrm{mm}$. transversi metatarsi 1, 2, 3" in reference to these same set of muscles, which unite metatarsal II with 1 , III with II, and V with III, respectively. While his mm. transversi metatarsi 1 and 2 are our $\mathrm{mm}$. intermetatarsales $\mathrm{I}$ and II, respectively, his $m$. transversus 3 requires further comments.

Ecker (1864) described a single m. interosseus uniting metatarsals III-V (= his $\mathrm{m}$. transversus 3 of his fig. 91). This muscle is, in fact, our $\mathrm{m}$. intermetatarsalis III and our $\mathrm{m}$ intermetatarsalis IV, which are partially fused. In several ranoids (this study), these two $\mathrm{mm}$. intermetatarsales are ventrally (i.e., superficially) fused, only separated by a thin raphe. This raphe is extremely reduced in some specimens to the extent that it appears to be a single muscle uniting metatarsals III and V. Furthermore, both muscles (our $\mathrm{mm}$. intermetatarsales III and IV) are attached to metatarsal IV dorsally to this raphe (Gaupp, 1895: 216, 1896: 214 [he refers to this raphe as an "inscriptio tendinea"]; this study). Considering this, the portion with origin on metatarsal $\mathrm{V}$ and insertion on metatarsal IV plus the raphe corresponds to our $\mathrm{m}$. intermetatarsalis IV, while the portion with origin on metatarsal IV plus the raphe and insertion on metatarsal III corresponds to our $\mathrm{m}$. intermetatarsalis III. In agreement with Perrin (1891: 20), this peculiar morphology may have led Dugès (1834) and Ecker (1864) to consider only a single $\mathrm{m}$. intermetatarsalis uniting metatarsals III and V.

M. LUMBRICALIS DIGITI IV: This muscle corresponds to our medial $\mathrm{m}$. lumbricalis brevis digiti IV.

M. FLEXOR BREVIS DIGITI IV: This muscle corresponds to our lateral $\mathrm{m}$. lumbricalis brevis digiti IV,

M. ADDUCTOR DIGITI V: This muscle corresponds to our medial $\mathrm{m}$. lumbricalis brevis digiti $\mathrm{V}$.

M, FLEXOR BREVIS DIGITI V: This muscle corresponds to our lateral $\mathrm{m}$. lumbricalis brevis digiti $\mathrm{V}$.

Perrin (1892)

M. FLÉCHISSEUR EXTERNE DES DOIGTS: The portion of this muscle that corresponds to our $\mathrm{mm}$. flexores breves superficiales is the lateral muscle of his figure 18 , labeled as 9.e.

M. FLÉCHISSEUR INTERNE DES DOIGTS: The portion of this muscle that corresponds to our m. tibialis posterior is the medial muscle, labeled as 9.d.

M. ROTATEUR DIRECT DU PIED: The portion of this muscle that corresponds to our $\mathrm{m}$. contrahentium caput longum is the medial muscle, while the lateral muscle corresponds to our $\mathrm{m}$. interosseus cruris; both muscles are labeled as 73 .

M. TARSO-FLÉCHISSEUR DES DOIGTS: The proximal and distal portions described by Perrin (1892) correspond to our m. flexor accessorius proximalis and $\mathrm{m}$. flexor accessorius distalis, respectively. 
Ribbing (1909)

M. INTEROSSEUS (OF DIGIT IV): Ribbing (1909: 72-73) considered our $\mathrm{m}$. abductor proprius digiti IV as part of our $\mathrm{m}$. intermetatarsalis IV.

Ribbing (1911)

M. FLEXOR BREVIS SUPERFICIALIS (OF DIGIT I): Ribbing (1911: 19) described a portion of his mm. flexores breves superficiales on digit I (which might correspond to our m. lumbricalis brevis hallucis). However, the superficial description along with the absence of figures precludes a confident interpretation of its identity. Furthermore, the data for Pipa pipa presented by Dunlap (1960: 35) and our observations in other species of the genus are conclusive regarding the absence of our $\mathrm{m}$. lumbricalis brevis hallucis in Pipa (see char. 14).

M. FLEXOR BREVIS SUPERFICIALIS (OF DIGIT II): Ribbing (1911: 19) described a portion of this muscle that inserts on the distal phalanx of digit II and other portion that inserts on the basal phalanx of digit II. They correspond to our $\mathrm{m}$. lumbricalis longus digiti II and our m. lumbricalis brevis digiti II, respectively.

M. FLEXOR BREVIS SUPERFICIALIS (OF DIGIT III): Ribbing (1911: 19) described a portion of this muscle that inserts on the second phalanx of digit III and other portion that inserts on metatarsal III. They correspond to our $\mathrm{m}$. lumbricalis longus digiti III and our $\mathrm{m}$. lumbricalis brevis digiti III, respectively. The identity of the muscle that inserts on metatarsal III agrees with the description of Dunlap (1960: 36) for Pipa pipa, and with our observations of other species of the genus.

M. FLEXOR BREVIS SUPERFICIALIS (OF DIGIT IV): Ribbing (1911: 19) described a portion of this muscle that inserts on the third phalanx of digit IV; it corresponds to our $\mathrm{m}$. lumbricalis longissimus digiti IV. It must be noted that this muscle may also be interpreted as the distal fleshy portion of the $\mathrm{m}$. lumbricalis longissimus digiti IV sensu Burton (2001) or as the main portion of the $\mathrm{m}$. lumbricalis longissimus digiti IV (in which case it is distally displaced). This particular morphology of the $\mathrm{m}$. lumbricalis longis- simus digiti IV agrees with the descriptions of Dunlap (1960: 38) for Pipa pipa, and our observations of other species of the genus.

A portion of this muscle corresponding to our $\mathrm{m}$. lumbricalis longus digiti IV was not specifically described by Ribbing (1911; 19). However, he stated that the same elements of this muscle group described by Gaupp (1896) for Pelophylax are also present in digit IV of Pipa (Pelophylax has our $\mathrm{m}$. lumbricalis longus digiti IV present). Furthermore, our m. lumbricalis longus digiti IV is present in Pipa pipa (Dunlap, 1960: 37-38) and other species of the genus (this study). Based on this, we denote it with a question mark in the synonym list of the $\mathrm{m}$. lumbricalis longus digiti IV.

The portion inserting on the basal phalanx of digit IV corresponds to our medial m. lumbricalis brevis digiti IV, while the portion inserting on metatarsal IV corresponds to our lateral $\mathrm{m}$. lumbricalis brevis digiti IV. This description agrees with Dunlap (1960: 36) for Pipa pipa, and with our observations in other species of the genus.

M. FLEXOR BREVIS SUPERFICIALIS (OF DIGIT V): The portion described by Ribbing $(1911 ; 19)$ inserting on the second phalanx corresponds to our $\mathrm{m}$. lumbricalis longus digiti V. Similar to the case for the $\mathrm{m}$. lumbricalis longissimus digiti IV (see discussion above), this muscle may be interpreted as the distal fleshy portion of the $\mathrm{m}$. lumbricalis longus digiti V sensu Burton (2001) or, alternatively, as the main portion of the $\mathrm{m}$. lumbricalis longus digiti $\mathrm{V}$ (in which case it is distally displaced). This particular morphology described by Ribbing (1911) agrees with Dunlap (1960: 38-39) for Pipa pipa, and with our observations in other species of the genus.

The portion of this muscle that inserts on metatarsal $\mathrm{V}$ and the basal phalanx of digit $\mathrm{V}$ probably correspond to our lateral $\mathrm{m}$. lumbricalis brevis digiti $\mathrm{V}$, while the medial $\mathrm{m}$. lumbricalis brevis digiti $\mathrm{V}$ is probably absent. The presence of a single muscle of the $\mathrm{mm}$. lumbricales breves in digit $V$ agrees with the descriptions of Dunlap (1960: 37) for Pipa pipa and with our observations in other species of the genus. 
M. FLEXOR MINIMUS DIGITI IV: The name and concept of the $\mathrm{mm}$. flexores digitorum minimi of Ribbing (1911) is equivalent to those employed in our study. However, Ribbing (1911: 20) stated that only the $\mathrm{m}$. flexor minimus of digit IV is present in his sample of Pipa cf. pipa, while Dunlap (1960: 42) reported it as absent in Pipa pipa. Although we did not study the foot musculature of Pipa pipa, the m. flexor minimus digiti IV is present in the other four species of the genus studied by us. At this point, further investigation is required to evaluate both the identity of the species studied by Ribbing (1911) as well as possible intraspecific variation.

M. INTERPHALANGEUS Digiti IV: Ribbing (1911: 20) generically stated that parts of the three interphalangei are present in Pipa cf. pipa. This statement refers to the three digits where these muscles usually occur: digits III-V. Our m. interphalangeus distalis digiti IV is absent in Pipa pipa (see Dunlap, 1960: 44), and it is also missing in other species of the genus (this study). Following this, the presence indicated by Ribbing (1911) might correspond to our interphalangeus proximalis digiti IV.

M. INTEROSSEUS (PORTION UNITING METATARSALS IV AND V): Ribbing (1911) combined our $\mathrm{m}$. intermetatarsalis IV and $\mathrm{m}$. abductor proprius digiti IV under this single name "interosseus"; both muscles are present in Pipa pipa (see Dunlap, 1960: 43, 45).

M. contrahentis pedis digiti V: The $\mathrm{m}$. contrahentis pedis digiti $\mathrm{V}$ is present in Pipa pipa (see Dunlap, 1960: 39-40), as well as in all nonneobatrachian species so far studied (see Character 16). The descriptions of Ribbing (1911) are not detailed enough to assess the identity of this muscle with confidence. Although considered absent by him, it might have been described under his $\mathrm{m}$. flexor brevis superficialis or $\mathrm{m}$. flexor brevis profundus. Possibly, the fact that the $\mathrm{m}$. contrahentis pedis digit $\mathrm{V}$ has a wide, fleshy insertion on metatarsal $\mathrm{V}$ (different to those of digits I-IV, characterized by a tendinous insertion on the basal phalanx) might have led Ribbing (1911) to consider it as part of his m. flexor brevis profundus, since this type of insertion characterizes this muscle group.

Dunlap (1960)

TENDO SUPERficialis PRAEHALluCIS: A similar term was employed by Dunlap (1960) only once (p. 33), "tendo superficialis praehallicus," possibly a misspelling.

M. LUMBricAlis bReVIS Digiti II: Dunlap (1960: 35) described in Heleioporus albopunctatus a lateral belly of this muscle that inserts on the distal interphalangeal joint of digit II. It corresponds to our $\mathrm{m}$. lumbricalis longus digiti II; see Burton (2001) for further comments.

M. CONTRAHENTIS HALLUCIS: This muscle corresponds to our $\mathrm{m}$. contrahentis pedis hallucis, with the exception of its report in Megaelosia goeldii and possibly in Hylodes cf. nasus (as Elosia nasus; see Dunlap, 1960:39). The muscle described for the former originates on metatarsal I (and not on the distal tarsals, a characteristic of the $\mathrm{mm}$. contrahentes pedis), and corresponds to our $\mathrm{m}$. flexor minimus hallucis; see Burton (2004: 210) and Discussion for further comments.

The identity of the muscle in Hylodes nasus described with this name is unclear. It might correspond to our $\mathrm{m}$. flexor minimus hallucis, to our $\mathrm{m}$. contrahentis pedis hallucis (in both situations with an atypical origin on metatarsal II), or to a novel muscle.

M. ABDUCTOR BREVIS PLANTARIS HALLUCIS: Dunlap (1960: 35) described, under the name $\mathrm{m}$. abductor brevis plantaris hallucis, a muscle with fleshy insertion on metatarsal I in Heleioporus albopunctatus and Limnodynastes peronii. Posteriorly, Burton (2001) defined and described the $\mathrm{m}$. adductor praehallucis as a muscle distinct from m. abductor brevis plantaris hallucis. Along this line, Burton (2001: 555) pointed out the particular insertion described by Dunlap (1960) in these two species, indicating that the m. abductor brevis plantaris hallucis usually inserts via a tendon, which is in agreement with Burton (2004), Blotto et al. (2017), and this study.

Since Heleioporus albopunctatus has the $\mathrm{m}$. abductor brevis plantaris hallucis absent and the 
m. adductor praehallucis present (Burton, 2001), the mention of Dunlap (1960) might correspond entirely to our $\mathrm{m}$. adductor praehallucis. Limnodynastes peronii has both muscles present (Burton, 2001; Blotto et al., 2017; this study), for which the portion with fleshy insertion described by Dunlap (1960) might correspond to our $\mathrm{m}$. adductor praehallucis.

At this point, it must be stated that the characteristic that unequivocally differentiates both muscles is the point of origin and not the type of insertion on metatarsal I. The origin of the $\mathrm{m}$. abductor brevis plantaris hallucis is restricted to the aponeurosis plantaris, while the origin of the $\mathrm{m}$. adductor praehallucis to the prehallux. The $\mathrm{m}$. abductor brevis plantaris hallucis may also have a fleshy insertion on metatarsal I, although less extended (this study).

Liem (1973)

M. LUMBRICALIS LONGUS DIGITI II: Liem (1973: 466) stated that Rheobatrachus silus has "only one slip of M. lumbricalis longus on 2nd toe." This reference might correspond to our m. lumbricalis brevis digiti II instead, since $R$. silus does not possess $\mathrm{m}$. lumbricalis longus digiti II (Davies and Burton, 1982; Burton, 2001; this study). Furthermore, the mention of "only one slip" by Liem (1973) suggests that he is referring to our $\mathrm{m}$. lumbricalis brevis, since more than one slip was described for this muscle (Dunlap, 1960), and not for the m. lumbricalis longus. Furthermore, the $\mathrm{m}$. lumbricalis longus was first defined and named as such only posteriorly by Burton (2001).

\section{Liem and Hosmer (1973)}

M. LUMBRICALIS LONGUS DIGITI II: Liem and Hosmer (1973: 440) stated that Taudactylus does not have the $\mathrm{m}$. lumbricalis longus digiti II, agreeing with Burton (2001) and our observations. Considering the possible confusion between the $\mathrm{m}$. lumbricalis brevis and $\mathrm{m}$. lumbricalis longus, noted above for Liem (1973), it is relevant to state here that the $\mathrm{m}$. lumbricalis bre- vis digiti 11 is present in Taudactylus (Burton, 2001; this study).

Davies and Burton (1982)

M. ABDUCTOR PRAEHALLUCIS: Also mentioned as "adductor brevis praehallucis" by Davies and Burton (1982: fig. 7d) and as "abductor brevis praehallucis" in their text.

\section{Burton (2001)}

M. LUMBRICALIS BREVIS DIGITI IV: The medial $\mathrm{m}$. lumbricalis brevis digiti IV is also named as "lumbricalis brevis medius digiti IV."

\section{Burton (2004)}

Superficial CUTANEOUS TENDONS: We take this expression partially from Burton (2004), referring to a set of tendons that extend from the aponeurosis plantaris and/or proximal ends of the tendines superficiales and insert on the connective tissue joining the integument of the subarticular tubercles to the medial and/or lateral metatarsophalangeal joints and/or the interphalangeal joints. These superficial cutaneous tendons vary in presence, distinctiveness (from thin or flat to sturdy, strong discrete tendons), in the specific digits in which they occur, and in their presence over the medial and/or lateral side of each digit (this study).

Burton (2004) made two references to these tendons. First (p. 212), under his section of "Superficial tendons," he says, "From a narrow, slightly raised strip of tendon in the aponeurosis plantaris, near the base of digit III, arises a set of three slender tendons. These pass distally along digits III-V, and insert on the dorsal surface of the skin of the subarticular tubercles at the metatarso-phalangeal joints." The second mention to these tendons is made under his state 2 of character 32 (p. 220), where he states "Tendo superficialis pro digiti III arising in part from $\mathrm{m}$. flexor digitorum brevis superficialis or the tendo superficialis pro digiti IV, and in part by a superficial tendon (from which also cutaneous tendons arise) that emerges from centrally on the plantar 
surface of the aponeurosis plantaris." We interpret that with the "cutaneous tendons" Burton (2004: 212) referred to the superficial tendons inserting on the skin, and we combine the expressions to form the term superficial cutaneous tendons.

Salgar et al. (2009)

M. OPPONENS HALLUCIS: Salgar et al. (2009: 60, fig. 6) described for Pristimantis bogotensis the presence of an $\mathrm{m}$. opponens hallucis with pennate origin from the proximal end of metatarsal $\mathrm{Il}$ and an insertion on the basal phalanx of digit I. Salgar et al. (2009: 66-67) recognized this morphology as unusual.

Based on its origin (metatarsal 11), position (laterally located on metatarsal I), and insertion (short tendon on the base of basal phalanx), it is not an $\mathrm{m}$. opponens hallucis (= our m. flexor hallucis accessorius). The $\mathrm{m}$, flexor hallucis accessorius is characterized by a more medial position, an origin from distal tarsals, aponeurosis plantaris, distal fused end of tibiale and fibulare, and/ or element $\mathrm{Y}$, and by a fleshy insertion on the plantar surface of metatarsal I (e.g., Dunlap, 1960; Burton, 1986, 2001, 2004; Blotto et al., 2017; this study).

The m. opponens hallucis of Salgar et al. (2009) probably corresponds to our $\mathrm{m}$. flexor minimus hallucis or to our m. contrahentis pedis hallucis, in both cases with an atypical origin on metatarsal 1I. The $\mathrm{m}$. flexor minimus hallucis originates from metatarsal $\mathrm{I}$, while the $\mathrm{m}$. contrahentis pedis hallucis originates from the distal tarsals, aponeurosis plantaris, distal fused end of tibiale and fibulare, and/or element Y (Burton, 2004; Blotto et al., 2017; this study). Interestingly, Dunlap (1960: 39) reported a muscle with similar morphology in Hylodes cf. nasus (as Elosia nasus).

Diogo and Molnar (2014)

MM. FLEXORES MINIMI DIGITORUM II-V: Diogo and Molnar (2014), following Dunlap (1960) for the musculature of Xenopus laevis, stated in table 8: "Flexores digitorum minimi ('flexores teretes digitorum'; 2 muscles to d.2-5)."
This statement does not specify which digits in this species have these $\mathrm{mm}$. flexores digitorum minimi. Muscles of this group are present in the digits III and IV of X. laevis and absent in the remaining digits (Dunlap, 1960: 42; this study); we follow this interpretation for the elaboration of the synonym list.

Diogo et al. (2018)

MM. FLEXORES MINIMI DIGITORUM II-V: Same comments as above for Diogo and Molnar (2014) apply here in regard to their statement in table 19.8. Furthermore, in their table 19.13 (p. 568 ) they stated that $X$. laevis has $\mathrm{mm}$. flexores minimi in digits II and IV. However, these muscles are present in digits III and IV (Dunlap, 1960: 42; this study).

\section{Dorsal Surface of the Foot}

\section{Dugès (1834)}

M. ASTRAGALO-SUS-PHALANGIEN DU DEUXIÈME DOIGT: We consider this muscle (number 183 of Dugès, 1834) to correspond to our $\mathrm{m}$. extensor brevis superficialis digiti II. However, Gaupp (1896: 218) considered that the calcanéosus-phalangien du deuxième doigt of Dugès (1834: muscle number 182) corresponds to the $\mathrm{m}$. extensor brevis superficialis digiti II (sensu both Gaupp, 1896, and our study); the descriptions and figures are not detailed enough for further interpretation.

M. ASTRAGALO-SUS-PHALANGIEN DU MÉDIUS: This muscle (number 181 of Dugès, 1834) might correspond to part of our m. extensor brevis superficialis digiti III or our $\mathrm{m}$. extensor brevis medius digiti III; descriptions and figures are not detailed enough at this point.

M. CALCANÉO-SUS-PHALANGIEN DU QUATRIÈME DOIGT AND M. SUS-CALCANÉO-PHALANGINIEN DU QUATRIÈME DOIGT: These muscles correspond to our medial and lateral $\mathrm{m}$. extensor brevis superficialis digiti IV, respectively.

M. ASTRAGALO-SUS-PHALANGETTIEN DU SECOND DOIGT: This muscle (number 217 of Dugès, 
1834) might correspond to a portion of our $\mathrm{m}$. extensor brevis medius digiti II, but it cannot be discarded a correspondence with part of our medial $\mathrm{m}$. dorsometatarsalis proximalis digiti II. Gaupp (1896:218) considered that the astragalosus-phalangien du deuxième doigt (number 183 of Dugès, 1834) corresponds to our $m$. extensor brevis medius digiti II; descriptions and figures are not detailed enough for further evaluation.

M. ASTRAGALO-EX-MÉTATARSIEN DU POUCE AND M. EX-TARSO-MÉTATARSIEN DU POUCE: These muscles correspond to the heads from element $\mathrm{Y}$ and prehallux of our $\mathrm{m}$. abductor brevis dorsalis hallucis, respectively.

M. MÉTATARSO-SUS-PHALANGIEN DU DEUXIÈME DOIGT AND M. MÉTATARSO-SUS-PHALANGETTIEN DU SECOND DOIGT: These muscles correspond to our medial and lateral $\mathrm{m}$. dorsometatarsalis proximalis digiti II, respectively.

M. MÉTATARSO-MÉTATARSIEN (OF DIGIT IV): See discussion above in the plantar surface section.

Ecker (1864)

M. EXTENSOR LONGUS DIGITI I: The portion of this muscle that corresponds to our m. extensor brevis superficialis hallucis is the one described by Ecker (1864: 132-133) as the "long head"; his "short head" corresponds to our $\mathrm{m}$. extensor brevis medius hallucis.

M. ABDUCTOR BREVIS DIGITI I AND M. EXTENSOR BREVIS DIGITI I: These muscles correspond to the heads of the prehallux and element $\mathrm{Y}$ of our $\mathrm{m}$. abductor brevis dorsalis hallucis, respectively.

M. INTEROSSEUS DORSALIS II: This muscle corresponds to our lateral $\mathrm{m}$. dorsometatarsalis hallucis proximalis. The medial muscle of our $\mathrm{m}$. dorsometatarsalis hallucis proximalis went undescribed.

MM. INTEROSSEI DORSALES III AND IV: These muscles correspond to our medial and lateral m. dorsometatarsalis proximalis digiti II, respectively.

MM. INTEROSSEI DORSALES V AND VI: These muscles correspond to our medial and lateral $\mathrm{m}$. dorsometatarsalis proximalis digiti III, respectively.
MM. INTEROSSEI DORSALES VII AND VIII: These muscles correspond to our medial and lateral $\mathrm{m}$. dorsometatarsalis proximalis digiti IV, respectively.

MM. INTEROSSEI DORSALES IX AND X, AND M. ABDUCTOR DIGITI V BREVIS: The former two muscles correspond to our medial and lateral $\mathrm{m}$. dorsometatarsalis proximalis digiti $\mathrm{V}$, respectively. Ecker (1864) also refers to our lateral $\mathrm{m}$. dorsometatarsalis proximalis digiti $\mathrm{V}$ as the $\mathrm{m}$. abductor digiti $\mathrm{V}$ brevis.

Perrin (1892)

M. EXTENSEUR SUPERFICIEL DE L'ERGOT AND M. EXTENSEUR SUPERFICIEL DE LA PREMIÈRE PHALANGE: Both muscles correspond to our $\mathrm{m}$. extensor brevis superficialis hallucis. The former corresponds to the portion inserting on the prehallux, while the $\mathrm{m}$. extenseur superficiel de la première phalange to the portion inserting on metatarsal I and metatarsophalangeal joint/basal phalanx of digit I.

M. EXTENSEUR DE LA QUATRIEME PHALANGE AND M. EXTENSEUR DE LA QUATRIÈME PHALANGINETTE: These muscles correspond to our medial and lateral $\mathrm{m}$. extensor brevis superficialis digiti IV, respectively.

M. ROTATEUR INVERSE DU PIED: The portion that corresponds to our $\mathrm{m}$. tarsalis anticus is the "posterior" portion, labeled as 92 ' in figures 22-23 of Perrin (1892).

Gaupp $(1895,1896)$

M. EXTENSOR BREVIS MEDIUS DIGITI IV: It corresponds to our m. extensor brevis superficialis digiti IV; see further comments in character 18 ,

Ribbing (1909)

EXTENSOR BREVIS SUPERFICIALIS (OF DIGIT IV): Ribbing (1909) studied Discoglossus, a genus in which our $\mathrm{m}$. extensor brevis medius digiti IV is present (see char. 18). Considering this, part of the muscle described for Discoglossus as the $\mathrm{m}$. extensor brevis superficialis with insertion on digit IV might correspond to our $\mathrm{m}$. extensor brevis medius digiti IV. See comments for his 
concept of the mm. extensores breves superficiales and profundi below under Ribbing's (1911) section.

\section{Ribbing (1911)}

GenERAL COMMENTS: For the elaboration of the synonym list, we interpret that Ribbing (1909, 1911) considered the $\mathrm{mm}$. extensores breves superficiales and medii of Gaupp (1896) as the $\mathrm{mm}$, extensores breves superficiales (thus, not establishing an explicit terminological distinction between both muscle groups), and the $\mathrm{mm}$. extensores breves profundi of Gaupp (1896) as his mm. extensores breves profundi (thus, not modifying Gaupp's concept, but see below for a possible problem regarding the identities in digit IV).

M. EXTENSOR BREVIS PROFUNDUS DIGITI IV: Some clarifications are required regarding the identity of $\mathrm{m}$. extensor brevis medius, as inferred from the footnotes 1 and 2, of Ribbing (1911: 22). First, the $m$. extensor brevis medius from the fibulare (sensu Ribbing; listed in his footnote 1) corresponds to our m. extensor brevis superficialis; only a single muscle is present in Pipa pipa and other species of the genus (Dunlap, 1960: 50; this study). Second, the m. extensor brevis medius from the tibiale (sensu Ribbing; footnote 2) might correspond to our medial m. dorsometatarsalis proximalis digiti IV with origin on the tibiale. The identity of the m. extensor brevis medius of digit IV with origin on the tibiale (sensu Ribbing) does not correspond to our $\mathrm{m}$. extensor brevis medius digiti IV. The $\mathrm{m}$. extensor brevis medius digiti IV is absent in Pipa pipa (see Dunlap, 1960: 55), and it is also absent in other species of the genus (see Character 18).

M. interosseus (oF Digit IV): See comments above in the plantar surface section.

Dunlap (1960)

General Comments: All discussion below refers to the presence or absence of $\mathrm{mm}$. dorsometatarsales distales in Chiromantis xerampelina and Espadarana prosoblepon (studied by Dunlap), since these muscles were only formally defined as a distinct muscle group later by Burton (1996) for the analogous muscles of the hand and by Faivovich (2002) for the muscles of the foot. Based on the description of the morphology of the $\mathrm{mm}$. extensores breves profundi done by Dunlap (1960) and the comparison with available data from these two species, it is possible to determine whether the muscles in some digits described by Dunlap (1960) correspond to our $\mathrm{mm}$. dorsometatarsales proximales or our $\mathrm{mm}$. dorsometatarsales distales.

Comparisons with data from Burton (2004) for Chiromantis xerampelina and Espadarana prosoblepon regarding the presence of $\mathrm{mm}$. dorsometatarsales distales are restricted to the digits III-V. For digits I-II, Burton (2004: 227) recorded together (i.e., within the same character state) the mm. extensores breves distales (= our $\mathrm{mm}$. dorsometatarsales distales) and the $\mathrm{mm}$. extensores breves profundi (= our $\mathrm{mm}$. dorsometatarsales proximales) with elongated fibers reaching the phalanx (which emulate the former; see Burton, 1998c: 614; Burton, 2004: 227). In consequence, it is not possible to make the distinction for the presence of $\mathrm{mm}$. dorsometatarsales distales and the $\mathrm{mm}$. dorsometatarsales proximales with elongated fibers in these two digits from Burton's (2004) data. Discussions for digits I-II are restricted to our observations in these two species.

M. EXTENSOR BREVIS PROFUNDUS HALLUCIS: Dunlap (1960: 55) reported a portion of the lateral $\mathrm{m}$, extensor brevis profundus hallucis with the fibers reaching the interphalangeal joint of digit I in Chiromantis xerampelina and Espadarana prosoblepon (as Centrolene). According to our observations in these two species, this portion corresponds to our lateral $\mathrm{m}$. dorsometatarsalis hallucis distalis.

M. EXTENSOR BREVIS PROFUNDUS DIGITI II: Dunlap (1960: 56) reported a portion of the lateral $\mathrm{m}$. extensor brevis profundus digiti II with the fibers reaching the interphalangeal joint of digit II in Espadarana prosoblepon. According to our observations, this portion corresponds to our lateral $\mathrm{m}$. dorsometatarsalis distalis digiti $\mathrm{Il}$. 
Dunlap (1960: 56) reported a portion of the lateral $\mathrm{m}$. extensor brevis profundus digiti II with the fibers reaching the interphalangeal joint of digit II in Chiromantis xerampelina. However, the specimen of C. xerampelina studied by us does not possess a lateral $\mathrm{m}$. dorsometatarsalis distalis digiti II, and the fibers of the lateral $\mathrm{m}$. dorsometatarsalis proximalis digiti II do not reach the phalanx. In consequence, it is not clear if the observation of Dunlap (1960) may be attributed to the presence of a lateral $\mathrm{m}$. dorsometatarsalis distalis digiti II, or to the presence of fibers of the lateral $\mathrm{m}$. dorsometatarsalis proximalis digiti 11 reaching the phalanx (implying polymorphism, since both conditions are absent in the specimen studied by us).

M. EXTENSOR BREVIS PROFUNDUS DIGITI III: Dunlap (1960: 57) described a portion of the lateral $\mathrm{m}$. extensor brevis profundus digiti III with origin "from the tendon of the lateral profundus and in part from the metatarso-digital joint" in Chiromantis xerampelina and Espadarana prosoblepon. This description corresponds to our lateral $\mathrm{m}$. dorsometatarsalis distalis digiti III, which is present in these two species (Burton, 2004; this study).

M. EXTENSOR BREVIS PROFUNDUS DIGITI IV: Dunlap (1960: 57) described a portion of the lateral $\mathrm{m}$. extensor brevis profundus digiti IV that "extends along the basal phalanx and tapes into a tendon only at the proximal interphalangeal joint" in Chiromantis xerampelina ("tapes" is probably a misspelling for "tapers," which is employed by Dunlap, 1960, in other equivalent descriptions). This portion corresponds to our lateral $\mathrm{m}$. dorsometatarsalis distalis digiti IV, which is present in this species (Burton, 2004; this study).

Dunlap (1960: 57) described a "digital" muscle associated with the lateral $\mathrm{m}$. extensor brevis profundus digiti IV for Espadarana prosoblepon. This "digital" muscle corresponds to our lateral $\mathrm{m}$. dorsometatarsalis distalis digiti $\mathrm{IV}$, which is present in this species (Burton, 2004; this study).

M. EXTENSOR BREVIS PROFUNDUS DIGITI V: Dunlap (1960: 58) described a fleshy portion of the medial $\mathrm{m}$. extensor brevis profundus digiti $\mathrm{V}$ over the basal phalanx of digit $\mathrm{V}$ in Chiromantis xerampelina. This portion corresponds to our medial $\mathrm{m}$. dorsometatarsalis distalis digiti $\mathrm{V}$, which is present in this species (Burton, 2004; this study).

Dunlap (1960: 58) described in Espadarana prosoblepon a "digital" muscle associated with the medial $\mathrm{m}$. extensor brevis profundus digiti V. This "digital" muscle corresponds to our medial $\mathrm{m}$. dorsometatarsalis distalis digiti $\mathrm{V}$, which is present in this species (Burton, 2004; this study).

Davies and Burton (1982)

M. EXTENSOR BREVIS MEDIUS DIGITI IV: It COYresponds to our $\mathrm{m}$. extensor brevis superficialis digiti IV; see comments above for Gaupp (1896).

M. EXTENSOR BREVIS SUPERFICIALIS DIGITI V: Davies and Burton (1982: 516) described a muscle under this name with origin on metatarsal V in Rheobatrachus silus; this origin fits with our $\mathrm{m}$. dorsometatarsalis proximalis digiti V. However, a long tendon of insertion to the basal phalanx of digit $\mathrm{V}$ is not a characteristic of the $\mathrm{mm}$. dorsometatarsales proximales (e.g., Dunlap, 1960; Burton, 2004; this study). The specimen studied by us does not possess $\mathrm{m}$. extensor brevis superficialis digiti $\mathrm{V}$, while the $\mathrm{mm}$. dorsometatarsales proximales of digit $\mathrm{V}$ are typical and without a long tendon of insertion as the one described by Davies and Burton (1982).

M. EXTENSOR BREVIS DORSALIS HALLUCIS: Davies and Burton (1982: 516) described it as a muscle that "originates from distal tarsalia and inserts penniform onto distal mediodorsal surface of metatarsus I." This insertion fits with our $\mathrm{m}$. abductor brevis dorsalis hallucis. However, an origin from a distal tarsal in the $\mathrm{m}$. abductor brevis dorsalis hallucis is unreported in the literature, and it was not observed in any specimen studied by us. A possible explanation is that the origin referred by Davies and Burton (1982) is not from the distal tarsalia, but from the element $\mathrm{Y}$ instead. The origin of part or the totality of the $\mathrm{m}$. abductor brevis dorsalis hallucis from element $\mathrm{Y}$ is very common (e.g., Dunlap, 1960: 58; this 
study), and it is present in the specimen of Rheobatrachus silus studied by us.

\section{Burton (1983)}

M. EXTENSOR BREVIS SUPERFICIALIS DIGITI IV AND M, EXTENSOR BREVIS MEDIUS DIGITI IV: Burton (1983: 321) mentioned an m. extensor brevis superficialis on digit $[\mathrm{V}$, and labeled it in his figure 14. Although it is not described and the figure is not detailed enough for us to identify it unequivocally, it possibly corresponds to our $\mathrm{m}$. extensor brevis superficialis digiti IV. The $\mathrm{mm}$. extensores breves medii of digit IV of Burton (1983) correspond to our mm. extensores breves superficiales.

M. EXTENSOR BREVIS DORSALIS HALLUCIS: Burton (1983: 321) described a muscle from:

the centrale preaxiale with a pinnate insertion distally onto metatarsus I and a long tendon of insertion to ultimate phalanx I. This muscle represents a fusion of the $M$. extensor brevis dorsalis hallucis and the medial slip of the $\mathrm{M}$. e. b. profundi hallucis (Fig. 14B, EBDH). In some specimens these two muscles are separate and typical.

His "M. e. b. profundi hallucis" corresponds to our medial $\mathrm{m}$. dorsometatarsalis hallucis proximalis. His $\mathrm{m}$. extensor brevis dorsalis hallucis corresponds to the portion of our $\mathrm{m}$. abductor brevis dorsalis hallucis with origin on element Y (as centrale preaxiale in Burton [1983]).

Fabrezi and Langone (2000)

General comments: Fabrezi and Langone (2000) studied the mm. extensores breves profundi (= our $\mathrm{mm}$. dorsometatarsales proximales) in Allophryne ruthveni. They described these muscles as having elongated fibers (i.e., reaching the phalanx), and were illustrated in their figure 7. They also stated that the $\mathrm{mm}$. extensores breves distales are absent ( $=$ our $\mathrm{mm}$. dorsometatarsales distales). Their reference to the $\mathrm{mm}$. extensores breves distales of the foot is probably analogous to the equivalent muscles described and defined for the hand by Burton (1996), since they were only formally defined as such for the foot by Faivovich (2002).

As done above for the hand, we evaluate here the presence of $\mathrm{mm}$. extensores breves distales (= our $\mathrm{mm}$. dorsometatarsales distales) and the presence of $\mathrm{mm}$. extensores breves profundi (= our $\mathrm{mm}$. dorsometatarsales proximales) with elongated fibers reaching the phalanx (which emulate the $\mathrm{mm}$. dorsometatarsales distales; see Burton, 1998c: 614; 2004: 227). Comparisons and discussions are performed based on our observations of Allophryne ruthveni and from the report of Burton (2004) for this species. As discussed above under Dunlap (1960), the comparisons regarding the presence of $\mathrm{mm}$. extensores breves distales ( $=$ our $\mathrm{mm}$. dorsometatarsales distales) from Burton's (2004) data are restricted to digits III-V.

M. EXTENSOR BREVIS PROFUNDUS HALLUCIS: We observed that the fibers of the medial $\mathrm{m}$. dorsometatarsalis hallucis proximalis do not reach the phalanx (contra Fabrezi and Langone, 2000). The fibers of the lateral dorsometatarsalis hallucis proximalis reach the phalanx and the $\mathrm{mm}$. dorsometatarsales distales of digit I are absent (agreeing with Fabrezi and Langone, 2000).

M. EXTENSOR BREVIS PROFUNDUS DIGITI II: We observed that the fibers of the medial and lateral $\mathrm{m}$. dorsometatarsalis proximalis digiti II do not reach the phalanx (contra Fabrezi and Langone, 2000; however, our data are not conclusive for the extension of the fibers of the lateral m. dorsometatarsalis proximalis). We also observed the lateral $\mathrm{m}$. dorsometatarsalis distalis present (contra Fabrezi and Langone, 2000).

Furthermore, their figure 7 shows a muscular mass over the medial side of the basal phalanx of digit II. The identity of this muscular portion needs corroboration, since no species of our sampling has a medial $\mathrm{m}$, dorsometatarsalis proximalis with fibers reaching the phalanx, nor a medial $\mathrm{m}$. dorsometatarsalis distalis digiti II.

M. EXTENSOR BREVIS PROFUNDUS DIGITI III: We observed that the fibers of the medial and lateral $\mathrm{m}$. dorsometatarsalis proximalis digiti III 
do not reach the phalanx, and only the lateral $\mathrm{m}$. dorsometatarsalis distalis digiti III is present (contra Fabrezi and Langone, 2000). This condition was reported by Burton (2004) and also observed by us.

Additionally, their figure 7 shows a muscular mass over the medial side of the basal and second phalanges of digit III. The identity of this muscular portion needs corroboration since no species of our sampling has a medial $\mathrm{m}$. dorsometatarsalis proximalis digiti III with fibers reaching the phalanx or a medial $\mathrm{m}$. dorsometatarsalis distalis digiti III. This suggests that this muscular mass of the figure of Fabrezi and Langone (2000) is not an m. dorsometatarsalis proximalis nor distalis. Except for intraspecific variation (unlikely in light of the previous comment), a putative and partial explanation is that the muscular mass on the medial side of the basal phalanx corresponds to the medial slip of the $\mathrm{m}$. interphalangeus digiti III, which is dorsally visible because its origin is dorsally extended in this species, occupying the dorsomedial surface of the basal phalanx (this study).

M. EXTENSOR BREVIS PROFUNDUS DIGITI IV: We observed that the fibers of the medial and lateral $\mathrm{m}$. dorsometatarsalis proximalis digiti IV do not reach the phalanx, and only the lateral $\mathrm{m}$. dorsometatarsalis distalis is present. This last muscle was also reported by Burton (2004).

The figure of Fabrezi and Langone (2000) shows a muscular mass over the medial side of the basal and second phalanges of digit IV. The identity of this muscular portion needs corroboration since no species of our sampling have a medial $\mathrm{m}$. dorsometatarsalis proximalis with fibers reaching the phalanx or a medial $\mathrm{m}$. dorsometatarsalis distalis. This suggests that the muscular mass of the figure of Fabrezi and Langone (2000) is not an $\mathrm{m}$. dorsometatarsalis proximalis or an $\mathrm{m}$. dorsometatarsalis distalis.

As discussed for digit III, and excluding intraspecific variation (unlikely in light of the previous comment), a putative explanation is that the muscular mass over the medial side of the phalanx corresponds to the medial slips of the $\mathrm{mm}$. interphalangei proximalis and distalis. These slips are dorsally visible because their origins are dorsally extended in this species, occupying the dorsomedial surface of the basal and second phalanx of digit IV (this study).

M. EXTENSOR BREVIS PROFUNDUS DIGITI V: We observed that the fibers of the medial and lateral $\mathrm{m}$. dorsometatarsalis proximalis digiti $\mathrm{V}$ do not reach the phalanx, but the medial and lateral $\mathrm{m}$. dorsometatarsalis distalis digiti $\mathrm{V}$ are present. This implies that the muscular mass figured by Fabrezi and Langone (2000) on the medial and lateral side of the phalanges may be interpreted as the medial and lateral $\mathrm{mm}$. dorsometatarsales distales, respectively. Burton (2004) scored the medial m. dorsometatarsalis distalis digiti $\mathrm{V}$ present and the lateral $\mathrm{m}$. dorsometatarsalis distalis digiti $\mathrm{V}$ absent, for which the lateral muscle might be subject to intraspecific variation.

\section{Burton (2001)}

M. EXTENSOR BREVIS SUPERFICIALIS DIGITI $\mathrm{V}$ : The $\mathrm{m}$. extensor brevis superficialis digiti $\mathrm{V}$ described by Burton (2001: 555) with origin from metatarsal $\mathrm{V}$ corresponds to our $\mathrm{m}$. dorsometatarsalis proximalis digiti $\mathrm{V}$; the muscle described as extensor brevis superficialis digiti $\mathrm{V}$ with a common origin with the $\mathrm{m}$. abductor brevis dorsalis digiti $\mathrm{V}$ (our $\mathrm{m}$. abductor digiti minimi) corresponds to our homonym muscle (Burton, 2001: 549, 555).

M. EXTENSOR BREVIS MEDIUS DIGITI IV: The concept of the $\mathrm{mm}$. extensores breves medii of digit IV of Burton (2001) follows Gaupp (1896) and corresponds entirely to our $\mathrm{mm}$. extensores breves superficiales of digit IV.

M. ABDUCTOR BREVIS DORSALIS HALLUCIS: Misspelled as “ $\mathrm{m}$. adductor brevis dorsalis hallucis" in its description (Burton, 2001: 548).

M. EXTENSOR BREVIS DORSALIS HALLUCIS: Burton (2001: 548) stated that this muscle is indistinguishable from the $\mathrm{m}$. abductor brevis dorsalis hallucis. As discussed above for Burton (1983), we consider that their m. extensor brevis dorsalis hallucis corresponds to a portion of our $\mathrm{m}$. abductor brevis dorsalis hallucis. 
M. EXTENSOR BREVIS PROFUNDUS DIGITI V: See comments above for the $\mathrm{m}$. extensor brevis superficialis digiti $\mathrm{V}$.

Burton (2004)

M. EXTENSOR BREVIS SUPERFICIALIS HALLUCIS, M. EXTENSOR BREVIS MEDIUS HALLUCIS, M. EXTENSOR BREVIS SUPERFICIALIS DIGITI II, AND M. EXTENSOR BREVIS MEDIUS DIGITI II: Burton (2004: 214) stated that the $\mathrm{m}$. extensor brevis superficialis hallucis and the $\mathrm{m}$. extensor brevis medius hallucis are fused and difficult to distinguish. He distinguished both muscles based on their distinct insertions, stating that the former inserts on the prehallux, the fascia between the prehallux and hallux, and by a tendon on the dorsum of metatarsal I, while the $\mathrm{m}$. extensor brevis medius hallucis inserts on the basal phalanx of digit I (note that we refer to the insertion on the basal phalanx described by Burton, 2004, as an insertion on the metatarsophalangeal joint).

Similarly, Burton (2004: 215) stated that the $\mathrm{m}$. extensor brevis superficialis and $\mathrm{m}$, extensor brevis medius of digit II are fused and difficult to distinguish. As for the muscles of the hallux, he differentiated the former by its insertion on metatarsal II, and the latter for its attachment to the basal phalanx of digit $\Pi$ (or metatarsophalangeal joint following our descriptive criteria).

We observed that the mm. extensores breves medii of digits I and $\Pi$ are absent in some species (including some species that were also studied by Burton) despite the presence of the insertions on the metatarsophalangeal joints of digits I and II. We interpret that these insertions on the metatarsophalangeal joints correspond to the $\mathrm{mm}$. extensores breves superficiales. We also observed the $\mathrm{mm}$. extensores breves superficiales inserting on their respective metatarsophalangeal joint in the presence of the $\mathrm{mm}$. extensores breves medii (e.g., pl. 5B). Furthermore, the m. extensor brevis medius digiti II also inserts on metatarsal II in some species (e.g., pl. 5C).

Consequently, the points of insertion are not appropriate to distinguish the $\mathrm{mm}$. extensores breves superficiales from the medii in digits I and II in Hylidae. Additional research is required to differentiate these muscles within Hylidae consistently and in a way that reflects homology.

Faivovich et al. (2005)

M. EXTENSOR BREVIS SUPERFICLALIS HALLUCIS, M. EXTENSOR BREVIS MEDIUS HALLUCIS, M. EXTENSOR BREVIS SUPERFICIALIS DIGITI II, AND M. EXTENSOR BREVIS MEDIUS DIGITI II: Same comments as above for Burton (2004).

Salgar et al. (2009)

M. EXTENSOR BREVIS DORSALIS HALLUCIS: This muscle is not described, and their figure 5 is not clear enough to state whether it corresponds to a portion of our medial $\mathrm{m}$. dorsometatarsalis hallucis proximalis (in which case a discrete tendon of insertion on the metatarsophalangeal joint and/or on interphalangeal joint/distal phalanx is expected), or to a portion of our $m$. abductor brevis dorsalis hallucis (in which case a fleshy insertion on metatarsal $I$ is expected).

Diogo and Ziermann (2014)

M. EXTENSOR BREVIS MEDiUS HALLUCIS: This muscle is considered present in Eleutherodactylus coqui by Diogo and Ziermann (2014: 98), as part of what they generically label as "Extensores breves medii + dorsometatarsales" in their figures $4 \mathrm{C}$ and $7 \mathrm{C}$. Portions of the $\mathrm{m}$. extensor brevis medius are easily confused with the $\mathrm{m}$. dorsometatarsalis proximalis and/or $\mathrm{m}$. extensor brevis superficialis, and the nature of these figures precludes an unequivocal assertion regarding its presence. The $\mathrm{m}$. extensor brevis medius hallucis is absent in the specimen of Eleutherodactylus coqui studied by us. Further studies are required to evaluate whether this muscle is present in some specimens of $E$. coqui.

M. EXTENSOR BREVIS MEDIUS DIGITI IV: This muscle is considered to be present by Diogo and Ziermann (2014: 98) in Eleutherodactylus coqui. This interpretation requires revision, since (1) there is confusion in the literature between the identities 
of this muscle and the m. extensor brevis superficialis digiti IV (see Dunlap [1960: 55] and several authors discussed here); and (2) the $\mathrm{m}$. extensor brevis medius digiti IV is absent in the specimen of E. coqui studied by us (see also char. 18).

Hoyos and Salgar (2016)

M. EXTENSOR BREVIS PROFUNDUS HALLUCIS: This muscle corresponds to our $\mathrm{m}$. dorsometatarsalis hallucis proximalis. However, one of the muscles labeled as such in their figure 4 (the label on its left side) corresponds to our $\mathrm{m}$. extensor brevis superficialis hallucis (possibly a labeling error). This incorrect label is the only mention attributable to the m. extensor brevis superficialis hallucis in their study.

M. EXTENSOR BREVIS PROFUNDUS DIGITI IV: The muscle labeled as such over the lateral side of the basal phalanx of digit IV in their figure 4 corresponds to our lateral $\mathrm{m}$. dorsometatarsalis distalis digiti IV. We corroborated this in the specimen of Dendropsophus luddeckei studied by us.

M. EXTENSOR BREVIS SUPERFICLALIS DIGITI V: The description of the muscle termed $\mathrm{m}$. extensor brevis superficialis digiti V (Hoyos and Salgar, 2016: 147), and labeled in their figure 4 as such, fits with our medial $\mathrm{m}$. dorsometatarsalis proximalis digiti V. This is also supported by the fact that our $\mathrm{m}$. extensor brevis superficialis digiti $\mathrm{V}$ is absent in the specimen of $D$. luddeckei studied by us.

Diogo et al. (2018)

M. EXTENSOR BREVIS MEDIUS DIGITI IV: Same comments as done above for Diogo and Ziermann (2014) regarding this name.

Names for Novel Tendons and Muscles not Addressed in Results or Discussion

SUPERFICIAL CUTANEOUS TENDONS (PALMAR SURFACE): Some species have a group of superficial cutaneous tendons, similar to those described for the foot by Burton (2004; see discussion and justification for the name above). We employ by analogy the same name as in the plantar surface.
M. INTERPHALANGEUS INDICIS AND M. INTERPHALANGEUS DIGITI III (OF THE HAND): We observed muscles of this group in digits II and III: an intermediate slip of the mm. interphalangei indicis and digiti III in Pipa parva, and a lateral slip of the $\mathrm{m}$, interphalangeus digiti III in Osteopilus septentrionalis.

M. INTERPHALANGEUS DIGITI II (OF THE FOOT): We report for the first time the $\mathrm{m}$. interphalangeus digiti II of the foot. We observed a lateral slip in Geocrinia victoriana, and an intermediate and lateral slip in one specimen of Odontophrynus americanus (MACN 48528; visible but not labeled in fig. 2 of Blotto et al., 2017).

M. INTERPHALANGEUS DISTALIS DIGITI V (OF THE FOOT): An intermediate slip with origin on the proximal interphalangeal joint and fleshy insertion on the distal half of the second phalanx of digit $\mathrm{V}$ was observed in Pipa carvalhoi. In analogy to the terminology of the mm. interphalangei of digit IV, the name of the muscle on basal phalanx is redefined to include the term "proximalis," resulting in $\mathrm{m}$. interphalangeus proximalis digiti V. 


\section{APPENDIX 4}

Data Matrix

\begin{tabular}{|c|c|c|c|c|c|c|c|c|c|c|c|c|c|c|c|c|c|c|c|c|}
\hline Species/Character & 1 & 2 & 3 & 4 & 5 & 6 & 7 & 8 & 9 & 10 & 11 & 12 & 13 & 14 & 15 & 16 & 17 & 18 & 19 & 20 \\
\hline Ascaphus truei & 0 & 1 & 1 & 0 & 1 & 0 & 0 & 1 & 0 & 1 & 0 & - & 1 & 1 & 0 & 1 & 1 & $0 \& 1$ & 0 & 0 \\
\hline Leiopelma hochstetteri & 0 & 0 & 1 & 0 & 1 & 0 & 0 & 1 & 0 & 1 & 0 & 0 & 1 & 1 & 0 & 1 & 1 & 0 & 0 & 0 \\
\hline Barbourula busuangensis & 0 & 0 & 1 & 0 & 1 & 0 & 0 & 0 & 2 & 1 & 0 & 0 & 0 & 1 & 0 & 1 & 1 & 0 & 0 & 0 \\
\hline Bombina orientalis & 0 & 0 & 1 & 1 & 1 & 0 & 0 & 0 & 2 & 1 & 0 & 0 & 0 & 1 & 0 & 1 & 1 & 0 & 0 & 0 \\
\hline Bombina variegata & 0 & 1 & 0 & 1 & 1 & 0 & 0 & 0 & 2 & 1 & 0 & 0 & 0 & 1 & 0 & 1 & 1 & 0 & 0 & 0 \\
\hline Bombina bombina & 0 & 0 & 1 & 0 & 1 & 0 & 0 & 0 & 2 & 1 & 0 & 0 & 0 & 1 & 0 & 1 & 1 & 0 & 0 & 0 \\
\hline Alytes obstetricans & 0 & 0 & 1 & 0 & 1 & 0 & 0 & 0 & 0 & 1 & 0 & - & 1 & 1 & 0 & 1 & 1 & 1 & 0 & 0 \\
\hline Discoglossus galganoi & 0 & 0 & 1 & 0 & 1 & 0 & 0 & 0 & 0 & 1 & 0 & 0 & 1 & 1 & 0 & 1 & 1 & 1 & 0 & 0 \\
\hline Discoglossus pictus & 0 & 0 & 1 & 0 & 1 & 0 & 0 & 0 & 0 & 1 & 0 & 0 & 1 & 1 & 0 & 1 & 1 & 1 & 0 & 0 \\
\hline Pipa parva & 0 & 0 & 0 & 1 & 1 & 0 & 0 & 0 & 0 & 1 & 0 & 0 & 0 & 0 & 0 & 1 & 1 & 0 & 0 & 0 \\
\hline Pipa carvalhoi & 0 & 0 & 0 & 1 & 1 & 0 & 0 & 0 & 0 & 1 & 0 & 0 & 0 & 0 & 0 & 1 & 1 & 0 & 0 & 0 \\
\hline Xenopus epitropicalis & 0 & 0 & 0 & 1 & 1 & 0 & 0 & 0 & 0 & 1 & 0 & 0 & 0 & 0 & 0 & 1 & 1 & 0 & 0 & 0 \\
\hline Xenopus muelleri & 0 & 0 & 1 & 1 & 1 & 0 & 0 & 0 & 0 & 1 & 0 & 0 & 0 & 0 & 0 & 1 & 1 & 0 & 0 & 0 \\
\hline Xenopus borealis & 0 & 0 & 1 & 0 & 1 & 0 & 0 & 0 & 0 & 1 & 0 & 0 & 0 & 0 & 0 & 1 & 1 & 0 & 0 & 0 \\
\hline Xenopus laevis & 0 & 0 & 1 & 0 & 1 & 0 & 0 & 0 & 0 & 1 & 0 & 1 & 0 & 0 & 0 & 1 & 1 & 0 & 0 & 0 \\
\hline Pseudhymenochirus merlini & 0 & 0 & 1 & 0 & 1 & 0 & 0 & 0 & 1 & 1 & 0 & - & 0 & 0 & 0 & 1 & 1 & 0 & 0 & 0 \\
\hline Hymenochirus curtipes & 0 & 0 & 1 & 0 & 1 & 0 & 0 & 0 & 1 & 1 & 0 & 0 & 0 & 0 & 0 & 1 & 1 & 0 & 0 & 0 \\
\hline Hymenochirus boettgeri & 0 & 0 & 1 & 0 & 1 & 0 & 0 & 0 & 1 & 1 & 0 & 0 & 0 & 0 & 0 & 1 & 1 & 0 & 0 & 0 \\
\hline Rhinophrynus dorsalis & 0 & 0 & 0 & 1 & 1 & 0 & 0 & 0 & 0 & 1 & 0 & 0 & 1 & 0 & 0 & 1 & 1 & 0 & 0 & 0 \\
\hline Scaphiopus couchii & 1 & 0 & 0 & 1 & 0 & 1 & 0 & 0 & 0 & 0 & 0 & 0 & 1 & 1 & 1 & 1 & 1 & 0 & 0 & 0 \\
\hline Scaphiopus holbrookii & 1 & 0 & 0 & 1 & 0 & 1 & 0 & 0 & 0 & 0 & 0 & 1 & 1 & 1 & 1 & 1 & 1 & 0 & 0 & 0 \\
\hline Scaphiopus hurterii & 1 & 0 & 0 & 1 & 0 & 1 & 0 & 0 & 0 & 0 & 0 & 1 & 1 & 1 & 1 & 1 & 1 & 0 & 0 & 0 \\
\hline Spea intermontana & 1 & 0 & 0 & 1 & 0 & 1 & 0 & 0 & 0 & 0 & 0 & 1 & 1 & 1 & 1 & 1 & 1 & 0 & 0 & 0 \\
\hline Spea bombifrons & 1 & 0 & 0 & 1 & 0 & 1 & 0 & 0 & 0 & 0 & 0 & 1 & 1 & 1 & 1 & 1 & 1 & 0 & 0 & 0 \\
\hline Spea hammondii & 1 & 0 & 0 & 1 & 0 & 1 & 0 & 0 & 0 & 0 & 0 & 0 & 1 & 1 & 1 & 1 & 1 & 0 & 0 & 0 \\
\hline Spea multiplicata & 1 & 0 & 0 & 1 & 0 & 1 & 0 & 0 & 0 & 0 & 0 & 1 & 1 & 1 & 1 & 1 & 1 & 0 & 0 & 0 \\
\hline Pelodytes ibericus & 1 & 0 & 0 & 1 & 0 & 1 & 0 & 0 & 0 & 0 & 1 & 1 & 1 & 1 & 0 & 1 & 1 & 0 & 0 & 0 \\
\hline Leptobrachella bourreti & 1 & 0 & 0 & 1 & 0 & 1 & 1 & 0 & 0 & 0 & 1 & 1 & 1 & 1 & 0 & 1 & 1 & 0 & 1 & 0 \\
\hline Leptobrachium lumadorum & 1 & 0 & 0 & 1 & 0 & 1 & 1 & 0 & 0 & 0 & 1 & 1 & 0 & 1 & 0 & 1 & 1 & 0 & 1 & 1 \\
\hline Oreolalax omeimontis & 1 & 0 & 0 & 1 & 0 & 1 & 1 & 0 & 0 & 0 & 1 & 1 & 1 & 1 & 0 & 1 & 1 & 0 & 1 & 1 \\
\hline Scutiger mammatus & 1 & 0 & 0 & 1 & 0 & 1 & 1 & 0 & 0 & 0 & 1 & 1 & 1 & 1 & 0 & 1 & 1 & 0 & 0 & 0 \\
\hline Megophrys carinense & 1 & 0 & 0 & 1 & 0 & 1 & 1 & 0 & 0 & 0 & 1 & 1 & 1 & 1 & 0 & 1 & 1 & 0 & 0 & 0 \\
\hline Megophrys aceras & 1 & 0 & 0 & 1 & 0 & 1 & 1 & 0 & 0 & 0 & 1 & 1 & 1 & 1 & 0 & 1 & 1 & 0 & 0 & 0 \\
\hline Megophrys edwardinae & 1 & 0 & 0 & 1 & 0 & 1 & 1 & 0 & 0 & 0 & 1 & 1 & 1 & 1 & 0 & 1 & 1 & 0 & 0 & 0 \\
\hline Pelobates fuscus & 1 & 0 & 0 & 1 & 0 & 1 & 0 & 0 & 0 & 0 & 1 & 1 & 1 & 1 & 0 & 1 & 1 & 0 & 0 & 0 \\
\hline Pelobates cultripes & 1 & 0 & 0 & 1 & 1 & 1 & 0 & 0 & 0 & 0 & 1 & 1 & 1 & 1 & 0 & 1 & 1 & 0 & 0 & 0 \\
\hline Pelobates varaldii & 1 & 0 & 0 & 1 & 1 & 1 & 0 & 0 & 0 & 0 & 1 & 1 & 1 & 1 & 0 & 1 & 1 & 0 & 0 & 0 \\
\hline
\end{tabular}


APPENDIX 4 continued

\begin{tabular}{|c|c|c|c|c|c|c|c|c|c|c|c|c|c|c|c|c|c|c|c|c|}
\hline Species/Character & 1 & 2 & 3 & 4 & 5 & 6 & 7 & 8 & 9 & 10 & 11 & 12 & 13 & 14 & 15 & 16 & 17 & 18 & 19 & 20 \\
\hline Hadromophryne natalensis & 1 & 1 & 1 & 0 & 1 & 0 & 0 & 0 & 0 & 1 & 0 & 1 & 1 & 1 & 0 & 0 & 1 & 0 & 0 & 0 \\
\hline Heleophryne orientalis & 1 & 1 & 0 & 1 & 1 & 1 & 0 & 1 & 0 & 1 & 0 & 1 & 1 & 1 & 0 & 0 & 1 & 0 & 0 & 0 \\
\hline Heleophryne purcelli & 1 & 1 & 1 & 1 & 1 & 0 & 0 & 1 & 0 & 1 & 0 & 0 & 1 & 1 & 0 & 0 & 1 & 0 & 0 & 0 \\
\hline Sooglossus sechellensis & 1 & 0 & 0 & 1 & 0 & 1 & 0 & 1 & 0 & 1 & 0 & 1 & 1 & 1 & 0 & 0 & 0 & 0 & 1 & 1 \\
\hline Sooglossus thomasseti & 1 & 0 & 0 & 1 & 0 & 1 & 0 & 1 & 0 & 1 & 0 & 1 & 1 & 1 & 0 & 0 & 0 & 0 & 1 & 1 \\
\hline Breviceps adspersus & 1 & 0 & 0 & 1 & 0 & 1 & 0 & 0 & 0 & 1 & 0 & 1 & 1 & 1 & 0 & 0 & 1 & 0 & 0 & 0 \\
\hline Hemisus marmoratus & 1 & 0 & 0 & 1 & 0 & 1 & 0 & 0 & 1 & 1 & 0 & 1 & 1 & 1 & 0 & 0 & 1 & 0 & 0 & 0 \\
\hline Arthroleptis variabilis & 1 & 0 & 0 & 1 & 0 & 1 & 0 & 0 & 1 & 1 & 0 & 1 & 1 & 1 & 0 & 0 & 1 & 0 & 1 & 1 \\
\hline Leptopelis natalensis & 1 & $?$ & 0 & 1 & $?$ & 1 & 0 & 0 & 1 & 1 & 0 & 1 & 1 & 1 & 0 & 0 & 1 & 0 & 1 & 1 \\
\hline Kassina senegalensis & 1 & 0 & 0 & 1 & 1 & 1 & 0 & 0 & 1 & 1 & 0 & 1 & 1 & 1 & 0 & 0 & 1 & 0 & 1 & 1 \\
\hline Afrixalus fulvovittatus & 1 & 0 & 0 & 1 & 1 & 1 & 0 & 0 & 1 & 1 & 0 & 1 & 1 & 1 & 0 & 0 & 1 & 0 & 1 & 1 \\
\hline Hyperolius semidiscus & 1 & 0 & 0 & 1 & 1 & 1 & 0 & 0 & 1 & 1 & 0 & 1 & 1 & 1 & 0 & 0 & 1 & 0 & 1 & 1 \\
\hline Phrynomantis bifasciatus & 1 & 0 & 0 & 1 & 1 & 1 & 0 & 0 & 0 & 1 & 0 & 1 & 0 & 1 & 0 & 0 & 1 & 0 & 0 & 0 \\
\hline Elachistocleis bicolor & 1 & 0 & 0 & 1 & 0 & 1 & 0 & 0 & 0 & 1 & 0 & 1 & 1 & 1 & 0 & 0 & 1 & 0 & 0 & 0 \\
\hline Ctenophryne geayi & 1 & 0 & 0 & 1 & 0 & 1 & 0 & 0 & 0 & 1 & 0 & 1 & 1 & 1 & 0 & 0 & 1 & 0 & 0 & 0 \\
\hline Kalophrynus sinensis & 1 & 0 & 0 & 1 & 0 & 1 & 0 & 0 & 0 & 1 & 0 & 1 & 1 & 1 & 0 & 0 & 1 & 0 & 0 & 0 \\
\hline Chaperina fusca & 1 & 0 & 0 & 1 & 0 & 1 & 0 & 1 & 0 & 1 & 0 & 1 & 1 & 1 & 0 & 0 & 1 & 0 & 0 & 0 \\
\hline Kaloula pulchra & 1 & 0 & 0 & 1 & 1 & 1 & 0 & 0 & 0 & 1 & 0 & 1 & 1 & 1 & 0 & 0 & 1 & 0 & 0 & 0 \\
\hline Uperodon systoma & 1 & 0 & 0 & 1 & 1 & 1 & 0 & 0 & 0 & 1 & 0 & 1 & 1 & 1 & 0 & 0 & 1 & 0 & 0 & 0 \\
\hline Micryletta inornata & 1 & 0 & 0 & 1 & 0 & 1 & 0 & 0 & 0 & 1 & 0 & 1 & 1 & 1 & 0 & 0 & 1 & 0 & 0 & 0 \\
\hline Microhyla heymonsi & 1 & 0 & 0 & 1 & 0 & 1 & 0 & 0 & 0 & 1 & 0 & 1 & 1 & 1 & 0 & 0 & 1 & 0 & 0 & 0 \\
\hline Glyphoglossus guttulatus & 1 & 0 & 0 & 1 & 0 & 1 & 0 & 0 & 0 & 1 & 0 & 1 & 1 & 1 & 0 & 0 & 1 & 0 & 0 & 0 \\
\hline Ptychadena nilotica & 1 & 0 & 0 & 1 & 0 & 1 & 0 & 0 & 1 & 1 & 0 & 1 & 1 & 1 & 0 & 0 & 1 & 0 & 0 & 0 \\
\hline Phrynobatrachus krefftii & 1 & 0 & 0 & 1 & 0 & 1 & 0 & 0 & 1 & 1 & 0 & 1 & 1 & 1 & 0 & 0 & 1 & 0 & 0 & 0 \\
\hline Tomopterna delalandii & 1 & 0 & 0 & 1 & 0 & 1 & 0 & 0 & 1 & 1 & 0 & 1 & 1 & 1 & 0 & 0 & 1 & 0 & 0 & 0 \\
\hline Cacosternum capense & 1 & 0 & 0 & 1 & 0 & 1 & 0 & 0 & 1 & 1 & 0 & 1 & 1 & 1 & 0 & 0 & 1 & 0 & 0 & 0 \\
\hline Microbatrachella capensis & 1 & 0 & 0 & 1 & 0 & 1 & 0 & 0 & 1 & 1 & 0 & 1 & 1 & 1 & 0 & 0 & 1 & 0 & 0 & 0 \\
\hline Cornufer guentheri & 1 & 0 & 0 & 1 & 0 & 1 & 0 & 0 & 1 & 1 & 0 & 1 & 1 & 1 & 0 & 0 & 1 & 0 & 0 & 0 \\
\hline Platymantis guentheri & 1 & 0 & 0 & 1 & 1 & 1 & 0 & 0 & 1 & 1 & 0 & 1 & 1 & 1 & 0 & 0 & 1 & 0 & 0 & 0 \\
\hline Occidozyga laevis & 1 & 0 & 0 & 1 & 1 & 1 & 0 & 0 & 1 & 1 & 0 & 1 & 1 & 1 & 0 & 0 & 1 & 0 & 0 & 0 \\
\hline Limnonectes woodworthi & 1 & 0 & 0 & 1 & 1 & 1 & 0 & 0 & 1 & 1 & 0 & 1 & 1 & 1 & 0 & 0 & 1 & 0 & 0 & 0 \\
\hline Hoplobatrachus rugulosus & 1 & 0 & 0 & 1 & 1 & 1 & 0 & 0 & 1 & 1 & 0 & 1 & 1 & 1 & 0 & 0 & 1 & 0 & 0 & 0 \\
\hline Fejervarya vittigera & 1 & 0 & 0 & 1 & 1 & 1 & 0 & 0 & 1 & 1 & 0 & 1 & 1 & 1 & 0 & 0 & 1 & 0 & 0 & 0 \\
\hline Sphaerotheca breviceps & 1 & 0 & 0 & 1 & 1 & 1 & 0 & 0 & 1 & 1 & 0 & 1 & 1 & 1 & 0 & 0 & 1 & 0 & 0 & 0 \\
\hline Chiromantis xerampelina & 1 & 0 & 0 & 1 & 1 & 1 & 0 & 0 & 1 & 1 & 0 & 1 & 1 & 1 & 0 & 0 & 1 & 0 & 0 & 0 \\
\hline Rhacophorus pardalis & 1 & 0 & 0 & 1 & 1 & 1 & 0 & 0 & 1 & 1 & 0 & 1 & 1 & 1 & 0 & 0 & 1 & 0 & 0 & 0 \\
\hline Polypedates leucomystax & 1 & 0 & 0 & 1 & 1 & 1 & 0 & 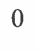 & 1 & 1 & 0 & 1 & 1 & 1 & 0 & 0 & 1 & 0 & 0 & 0 \\
\hline Staurois natator & 1 & 0 & 0 & 1 & 1 & 1 & 0 & 0 & 1 & 1 & 0 & 1 & 1 & 1 & 0 & 0 & 1 & 0 & 0 & 0 \\
\hline Sanguirana sanguinea & 1 & 0 & 0 & 1 & 1 & 1 & 0 & 0 & 1 & 1 & 0 & 1 & 1 & 1 & 0 & 0 & 1 & 0 & 0 & 0 \\
\hline
\end{tabular}


APPENDIX 4 continued

\begin{tabular}{|c|c|c|c|c|c|c|c|c|c|c|c|c|c|c|c|c|c|c|c|c|}
\hline Species/Character & 1 & 2 & 3 & 4 & 5 & 6 & 7 & 8 & 9 & 10 & 11 & 12 & 13 & 14 & 15 & 16 & 17 & 18 & 19 & 20 \\
\hline Lithobates warszewitschii & 1 & 0 & 0 & 1 & 1 & 1 & 0 & 0 & 2 & 1 & 0 & 1 & 1 & 1 & 0 & 0 & 1 & 0 & 0 & 0 \\
\hline Odorrana versabilis & 1 & 0 & 0 & 1 & 1 & 1 & 0 & 0 & 1 & 1 & 0 & 1 & 1 & 1 & 0 & 0 & 1 & 0 & 0 & 0 \\
\hline Calyptocephalella gayi & 1 & 1 & 1 & . & 1 & 1 & 0 & 0 & 0 & 1 & 0 & 1 & 1 & 1 & 0 & 0 & 1 & 0 & 0 & 0 \\
\hline Neobatrachus aquilonius & 1 & 0 & 0 & 1 & 1 & 0 & 0 & 0 & 0 & 1 & 0 & 1 & 1 & 1 & 0 & 0 & 1 & 0 & 1 & 1 \\
\hline Adelotus brevis & 1 & 0 & 0 & 1 & 1 & 0 & 0 & 0 & 0 & 1 & 0 & 1 & 1 & 1 & 0 & 0 & 1 & 0 & 0 & 0 \\
\hline Limnodynastes peronii & 1 & 0 & 0 & 1 & 1 & 0 & 0 & c & 0 & 1 & 0 & 1 & 1 & 1 & 0 & 0 & 1 & 0 & 0 & 0 \\
\hline Philoria frost $i$ & 1 & 0 & 0 & 1 & 1 & 0 & 0 & 0 & 0 & 1 & 0 & 1 & 1 & 1 & 0 & 0 & 1 & 0 & 0 & 0 \\
\hline Notaden bennettii & 1 & 0 & 0 & 1 & 1 & 0 & 0 & 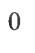 & 0 & 1 & 0 & 1 & 1 & 1 & 0 & 0 & 1 & 0 & 0 & 0 \\
\hline Platyplectrum ornatum & 1 & 0 & 0 & 1 & 1 & 1 & 0 & 0 & 0 & 1 & 0 & 1 & 1 & 1 & 0 & 0 & 1 & 0 & 0 & 0 \\
\hline Lechriodus melanopyga & 1 & 0 & 0 & 1 & 1 & 1 & 0 & 0 & 0 & 1 & 0 & 1 & 1 & 1 & 0 & 0 & 1 & 0 & 0 & 0 \\
\hline Rheobatrachus silus & 1 & 0 & 0 & 1 & 1 & 0 & 0 & 0 & 0 & 1 & 0 & 1 & 1 & 1 & 0 & 0 & 1 & 0 & 0 & 0 \\
\hline Mixophyes iteratus & 1 & 0 & 0 & 1 & 1 & 0 & 0 & 0 & 0 & 1 & 0 & 1 & 1 & 1 & 0 & 0 & 1 & 0 & 0 & 0 \\
\hline Taudactylus diurnus & 1 & 0 & 0 & 1 & 1 & 0 & 0 & 0 & 0 & 1 & 0 & 1 & 1 & 1 & 0 & 0 & 1 & 0 & 0 & 0 \\
\hline Uperoleia laevigata & 1 & 0 & 0 & 1 & 1 & 0 & 0 & 0 & 0 & 1 & 0 & 1 & 1 & 1 & 0 & 0 & 1 & 0 & 0 & 0 \\
\hline Pseudophryne coriace & 1 & 0 & 0 & 1 & 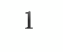 & 0 & 0 & 0 & 0 & 1 & 0 & 1 & 0 & 1 & 0 & 0 & 1 & 0 & 0 & 0 \\
\hline Crinias & 1 & 0 & 0 & 1 & 1 & 0 & 0 & 0 & 0 & 1 & 0 & 1 & 0 & 1 & 0 & 0 & 1 & 0 & 0 & 0 \\
\hline Geocrinia victoriana & 1 & 0 & - & - & 1 & 0 & 0 & 0 & 0 & 1 & 0 & 1 & 1 & 1 & 0 & 0 & 1 & 0 & 0 & 0 \\
\hline Ischnocnema guentheri & 1 & 0 & 0 & 1 & . & 1 & 0 & 0 & 1 & 1 & 0 & 1 & 1 & 1 & 0 & 0 & 1 & 0 & 0 & 0 \\
\hline Eleutherodactylus coqui & 1 & 0 & 0 & 1 & 0 & 1 & 0 & 0 & 1 & 1 & 0 & 1 & 1 & 1 & 0 & 0 & 1 & 0 & 0 & 0 \\
\hline Craugastor fitzingeri & 1 & 0 & 0 & 1 & 0 & 1 & 0 & 0 & 1 & 1 & 0 & 1 & 1 & 1 & 0 & 0 & 1 & 0 & 0 & 0 \\
\hline Haddadus binotatus & 1 & 0 & 0 & 1 & 0 & 1 & 0 & 0 & 1 & 1 & 0 & 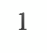 & 1 & 1 & 0 & 0 & 1 & 0 & 0 & 0 \\
\hline Barycholos ternetzi & 1 & 0 & 0 & 1 & 0 & 1 & 0 & 0 & 1 & 1 & 0 & 1 & 1 & 1 & 0 & 0 & 1 & 0 & 0 & 0 \\
\hline Pristimantis buckleyi & 1 & 0 & 0 & 1 & 0 & 1 & 0 & 0 & 1 & 1 & 0 & 1 & 1 & 1 & 0 & 0 & 1 & 0 & 0 & 0 \\
\hline Oreobates barituensis & 1 & 0 & 0 & 1 & 0 & 1 & 0 & 0 & 2 & 1 & 0 & 1 & 1 & 1 & 0 & 0 & 1 & 0 & 0 & 0 \\
\hline Flectonotus pygmaeus & 1 & 0 & 0 & 1 & 0 & 1 & 0 & 0 & 1 & 1 & 0 & 1 & 1 & 1 & 0 & 0 & 1 & 0 & 0 & 0 \\
\hline Hemiphractus pr & 1 & 0 & 0 & 1 & 0 & 1 & 0 & 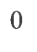 & 1 & 1 & 0 & 1 & 1 & 1 & 0 & 0 & 1 & 0 & 0 & 0 \\
\hline Stefania evansi & 1 & , & 0 & 1 & 0 & 1 & 0 & 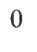 & 1 & 1 & 0 & , & 1 & 1 & 0 & 0 & 1 & 0 & 0 & 0 \\
\hline Gastrotheca christiani & 1 & 0 & 0 & 1 & 0 & 1 & 0 & 0 & 1 & 1 & 0 & $?$ & 1 & 1 & 0 & 0 & 1 & 0 & 0 & 0 \\
\hline Ceratophrys cranwelli & 1 & 0 & 0 & 1 & 0 & 1 & 0 & 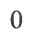 & 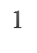 & 1 & 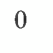 & 1 & 1 & 1 & 0 & 0 & 1 & 0 & 0 & 0 \\
\hline Chacophrys pierottii & 1 & 0 & 0 & 1 & 0 & 1 & 0 & 0 & 1 & 1 & 0 & 1 & 1 & 1 & 0 & 0 & 1 & 0 & 0 & 0 \\
\hline Lepidobatrachus laevis & 1 & 0 & 0 & 1 & 0 & 1 & 0 & 0 & 1 & 1 & 0 & 1 & 1 & 1 & 0 & 0 & 1 & 0 & 0 & 0 \\
\hline Proceratophrys boiei & 1 & 0 & 0 & 1 & $0 \& 1$ & 1 & 0 & 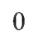 & 1 & 1 & 0 & 1 & 1 & 1 & 0 & 0 & 1 & 0 & 0 & 0 \\
\hline Macrogenioglottus alipioi & 1 & 0 & 0 & 1 & 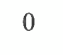 & 1 & 0 & 0 & 1 & 1 & 0 & 1 & 1 & 1 & 0 & 0 & 1 & 0 & 0 & 0 \\
\hline Odontophrynus achalensis & 1 & 0 & 0 & 1 & 1 & 1 & 0 & 0 & 1 & 1 & . & 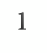 & 1 & 1 & 0 & 0 & 1 & 0 & 0 & 0 \\
\hline Telmatobius oxycephalus & 1 & 0 & 0 & 1 & 0 & 1 & 0 & 0 & 1 & 1 & 0 & 1 & 1 & 1 & 0 & 0 & 1 & 0 & 0 & 0 \\
\hline Rhinoderma darwinii & 1 & 0 & 0 & 1 & 0 & 1 & 0 & 0 & 1 & 1 & 0 & 1 & 1 & 1 & 0 & 0 & 1 & 0 & 0 & 0 \\
\hline Insuetophrynus acarpicus & 1 & 0 & 0 & 1 & 0 & 1 & 0 & 0 & 1 & 1 & 0 & 1 & 1 & 1 & 0 & 0 & 1 & 0 & 0 & 0 \\
\hline Cycloramphus boraceiensis & 1 & 0 & 0 & 1 & 0 & 1 & 0 & 0 & 1 & 1 & 0 & 1 & 1 & 1 & 0 & 0 & 1 & 0 & 0 & 0 \\
\hline Thoropa taophora & 1 & 0 & 0 & 1 & 0 & 1 & 0 & 0 & 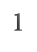 & 1 & 0 & 1 & 1 & 1 & 0 & 0 & 1 & 0 & 0 & 0 \\
\hline Crossodactylus schmidti & 1 & 0 & 0 & 1 & 0 & 1 & 0 & 0 & 1 & 1 & 0 & 1 & 1 & 1 & 0 & 0 & 1 & 0 & 0 & 0 \\
\hline
\end{tabular}


APPENDIX 4 continued

\begin{tabular}{|c|c|c|c|c|c|c|c|c|c|c|c|c|c|c|c|c|c|c|c|c|}
\hline Species/Character & 1 & 2 & 3 & 4 & 5 & 6 & 7 & 8 & 9 & 10 & 11 & 12 & 13 & 14 & 15 & 16 & 17 & 18 & 19 & 20 \\
\hline Hylodes phyllodes & 1 & 0 & 0 & 1 & 0 & 1 & 0 & 0 & 1 & 1 & 0 & 1 & 1 & 1 & 0 & 0 & 1 & 0 & 0 & 0 \\
\hline Limnomedusa macroglossa & 1 & 0 & 0 & 1 & 0 & 1 & 0 & 0 & 1 & 1 & 0 & 1 & 1 & 1 & 0 & 0 & 1 & 0 & 0 & 0 \\
\hline Alsodes neuquensis & 1 & 0 & 0 & 1 & 0 & 1 & 0 & 0 & 1 & 1 & 0 & 1 & 1 & 1 & 0 & 0 & 1 & 0 & 0 & 0 \\
\hline Eupsophus roseus & 1 & 0 & 0 & 1 & 0 & 1 & 0 & 0 & 0 & 1 & 0 & 1 & 1 & 1 & 0 & 0 & 1 & 0 & 0 & 0 \\
\hline Hylorina sylvatica & 1 & 0 & 0 & 1 & 0 & 1 & 0 & 0 & 1 & 1 & 0 & 1 & 1 & 1 & 0 & 0 & 1 & 0 & 0 & 0 \\
\hline Batrachyla leptopus & 1 & 0 & 0 & 1 & 0 & 1 & 0 & 0 & 1 & 1 & 0 & 0 & 1 & 1 & 0 & 0 & 1 & 0 & 0 & 0 \\
\hline Chaltenobatrachus grandisonae & 1 & 0 & 0 & 1 & 0 & 1 & 0 & 0 & 1 & 1 & 0 & 0 & 1 & 1 & 0 & 0 & 1 & 0 & 0 & 0 \\
\hline Atelognathus patagonicus & 1 & 0 & 0 & 1 & 0 & 1 & 0 & 0 & $1 \& 2$ & 1 & 0 & 1 & 1 & 1 & 0 & 0 & 1 & 0 & 0 & 0 \\
\hline Phyllomedusa sauvagii & 1 & 0 & 0 & 1 & 0 & 1 & 0 & 0 & 1 & 1 & 0 & 1 & 1 & 1 & 0 & 0 & 1 & 0 & 0 & 0 \\
\hline Ranoidea eucnemis & 1 & 0 & 0 & 1 & 0 & 1 & 0 & 0 & 1 & 1 & 0 & - & 1 & 1 & 0 & 0 & 1 & 0 & 0 & 0 \\
\hline Aplastodiscus perviridis & 1 & 0 & 0 & 1 & 0 & 1 & 0 & 0 & 1 & 1 & 0 & 1 & 1 & 1 & 0 & 0 & 1 & 0 & 0 & 0 \\
\hline Boana cordobae & 1 & 0 & 0 & 1 & 0 & 1 & 0 & 0 & 1 & 1 & 0 & 1 & 1 & 1 & 0 & 0 & 1 & 0 & 0 & 0 \\
\hline Trachycephalus typhonius & 1 & 0 & 0 & 1 & 0 & 1 & 0 & 0 & 1 & 1 & 0 & 1 & 1 & 1 & 0 & 0 & 1 & 0 & 0 & 0 \\
\hline Phyllodytes luteolus & 1 & 0 & 0 & 1 & 0 & 1 & 0 & 0 & 1 & 1 & 0 & 1 & 1 & 1 & 0 & 0 & 1 & 0 & 0 & 0 \\
\hline Acris crepitans & 1 & 0 & 0 & 1 & 0 & 1 & 0 & 0 & 1 & 1 & 0 & - & 1 & 1 & 0 & 0 & 1 & 0 & 0 & 0 \\
\hline Triprion petasatus & 1 & 0 & 0 & 1 & 0 & 1 & 0 & 0 & 1 & 1 & 0 & 1 & 1 & 1 & 0 & 0 & 1 & 0 & 0 & 0 \\
\hline Sphaenorhynchus lacteus & 1 & 0 & 0 & 1 & 0 & 1 & 0 & 0 & 1 & 1 & 0 & 1 & 1 & 1 & 0 & 0 & 1 & 0 & 0 & 0 \\
\hline Xenohyla truncata & 1 & 0 & 0 & 1 & 0 & 1 & 0 & 0 & 1 & 1 & 0 & 1 & 1 & 1 & 0 & 0 & 1 & 0 & 0 & 0 \\
\hline Dendropsophus minutus & 1 & 0 & 0 & 1 & 0 & 1 & 0 & 0 & 1 & 1 & 0 & $0 \& 1$ & 1 & 1 & 0 & 0 & 1 & 0 & 0 & 0 \\
\hline Scinax granulatus & 1 & 0 & 0 & 1 & 0 & 1 & 0 & 0 & 1 & 1 & 0 & 1 & 1 & 1 & 0 & 0 & 1 & 0 & 0 & 0 \\
\hline Scarthyla goinorum & 1 & 0 & 0 & 1 & 0 & 1 & 0 & 0 & 1 & 1 & 0 & 1 & 1 & 1 & 0 & 0 & 1 & 0 & 0 & 0 \\
\hline Pseudis minuta & 1 & 0 & 0 & 1 & 0 & 1 & 0 & 0 & 1 & 1 & 0 & 1 & 1 & 1 & 0 & 0 & 1 & 0 & 0 & 0 \\
\hline Allophryne ruthveni & 1 & 0 & 0 & 1 & 0 & 1 & 0 & 0 & 1 & 1 & 0 & 0 & 1 & 1 & 0 & 0 & 1 & 0 & 0 & 0 \\
\hline Espadarana prosoblepon & 1 & 0 & 0 & 1 & 0 & 1 & 0 & 0 & 1 & 1 & 0 & 1 & 1 & 1 & 0 & 0 & 1 & 0 & 0 & 0 \\
\hline Vitreorana uranoscopa & 1 & 0 & 0 & 1 & 0 & 1 & 0 & 0 & 1 & 1 & 0 & 0 & 1 & 1 & 0 & 0 & 1 & 0 & 0 & 0 \\
\hline Paratelmatobius poecilogaster & 1 & 0 & 0 & 1 & 0 & 1 & 0 & 0 & 1 & 1 & 0 & 1 & 1 & 1 & 0 & 0 & 1 & 0 & 0 & 0 \\
\hline Leptodactylus latinasus & 1 & 0 & 0 & 1 & 0 & 1 & 0 & 0 & 1 & 1 & 0 & 1 & 1 & 1 & 0 & 0 & 1 & 0 & 0 & 0 \\
\hline Pseudopaludicola falcipes & 1 & 0 & 0 & 1 & 0 & 1 & 0 & 0 & 1 & 1 & 0 & 1 & 1 & 1 & 0 & 0 & 1 & 0 & 0 & 0 \\
\hline Physalaemus biligonigerus & 1 & 0 & 0 & 1 & 0 & 1 & 0 & 0 & 1 & 1 & 0 & 1 & 1 & 1 & 0 & 0 & 1 & 0 & 0 & 0 \\
\hline Pleurodema kriegi & 1 & 0 & 0 & 1 & 0 & 1 & 0 & 0 & 1 & 1 & 0 & 1 & 1 & 1 & 0 & 0 & 1 & 0 & 0 & 0 \\
\hline Mannophryne herminae & 1 & 0 & 0 & 1 & 0 & 1 & 0 & 0 & 1 & 1 & 0 & 1 & 1 & 1 & 0 & 0 & 1 & 0 & 0 & 0 \\
\hline Adelphobates galactonotus & 1 & 0 & 0 & 1 & 0 & 1 & 0 & 0 & 1 & 1 & 0 & 1 & 1 & 1 & 0 & 0 & 1 & 0 & 0 & 0 \\
\hline & 1 & 0 & 0 & 1 & 0 & 1 & 0 & 0 & 1 & 1 & 0 & 1 & 1 & 1 & 0 & 0 & 1 & 0 & 0 & 0 \\
\hline Atelopus pastuso & 1 & 0 & 0 & 1 & 1 & 1 & 0 & 0 & 1 & 1 & 0 & 0 & 1 & 1 & 0 & 0 & 1 & 0 & 0 & 0 \\
\hline Dendrophryniscus leucomystax & 1 & 0 & 0 & 1 & 0 & 1 & 0 & 0 & 1 & 1 & 0 & 0 & 1 & 1 & 0 & 0 & 1 & 0 & 0 & 0 \\
\hline Amazophrynella minuta & 1 & 0 & 0 & 1 & 0 & 1 & 0 & 1 & 1 & 1 & 0 & 0 & 1 & 1 & 0 & 0 & 1 & 0 & 0 & 0 \\
\hline Nannophryne variegata & 1 & 0 & 0 & 1 & 0 & 1 & 0 & 0 & 2 & 1 & 0 & 1 & 1 & 1 & 0 & 0 & 1 & 0 & 0 & 0 \\
\hline Anaxyrus woodhousii & 1 & 0 & 0 & 1 & 0 & 1 & 0 & 0 & 1 & 1 & 0 & $0 \& 1$ & 1 & 1 & 0 & 0 & 1 & 0 & 0 & 0 \\
\hline Rhinella fernandezae & 1 & 0 & 0 & 1 & 0 & 1 & 0 & 0 & 1 & 1 & 0 & 1 & 1 & 1 & 0 & 0 & 1 & 0 & 0 & 0 \\
\hline
\end{tabular}




\section{APPENDIX 5}

\section{Ancestral Character State Reconstructions}

Character 1. M. flexor indicis superficialis proprius

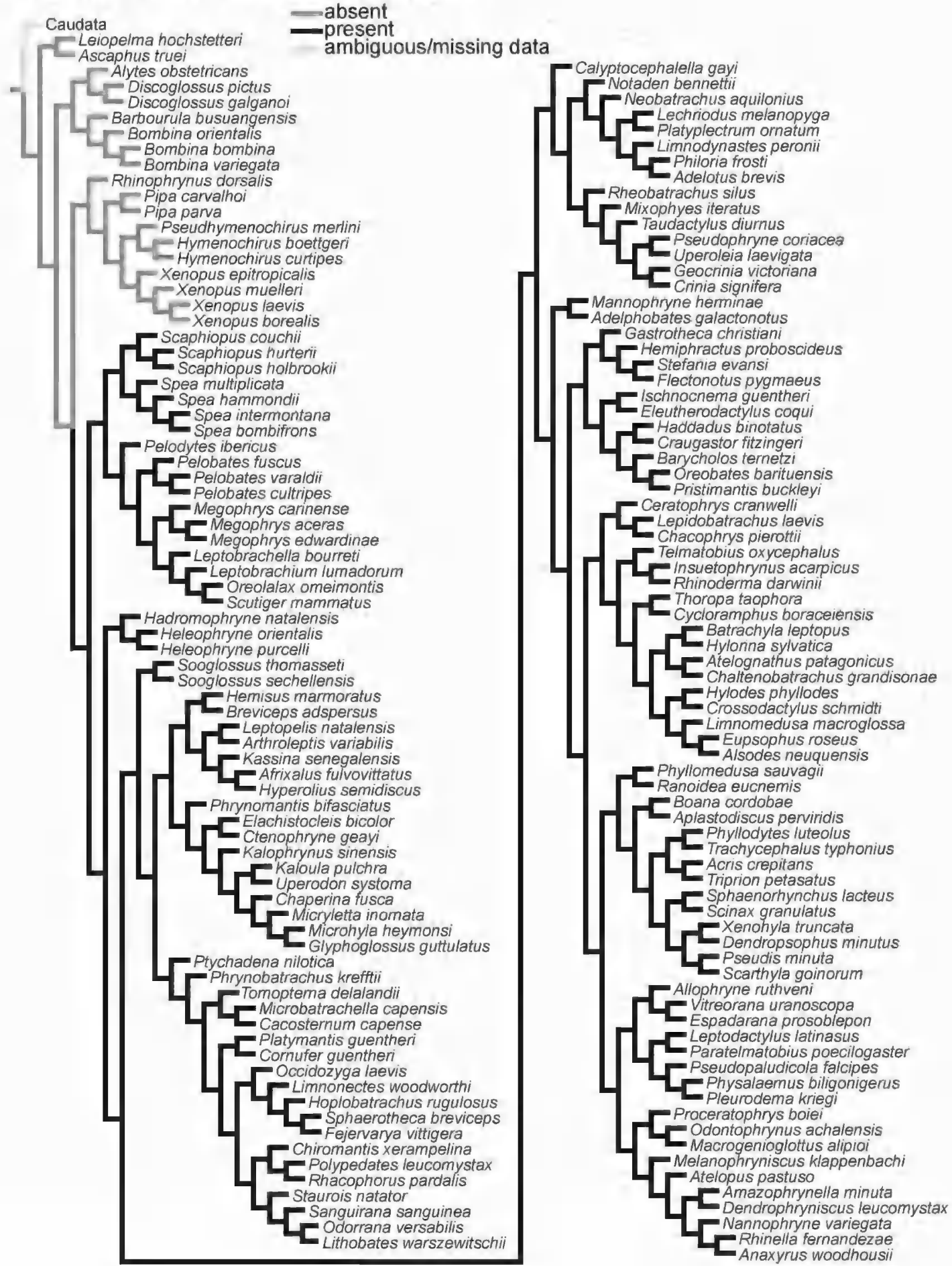


APPENDIX 5 continued

Character 2. M. lumbricalis longus digiti III

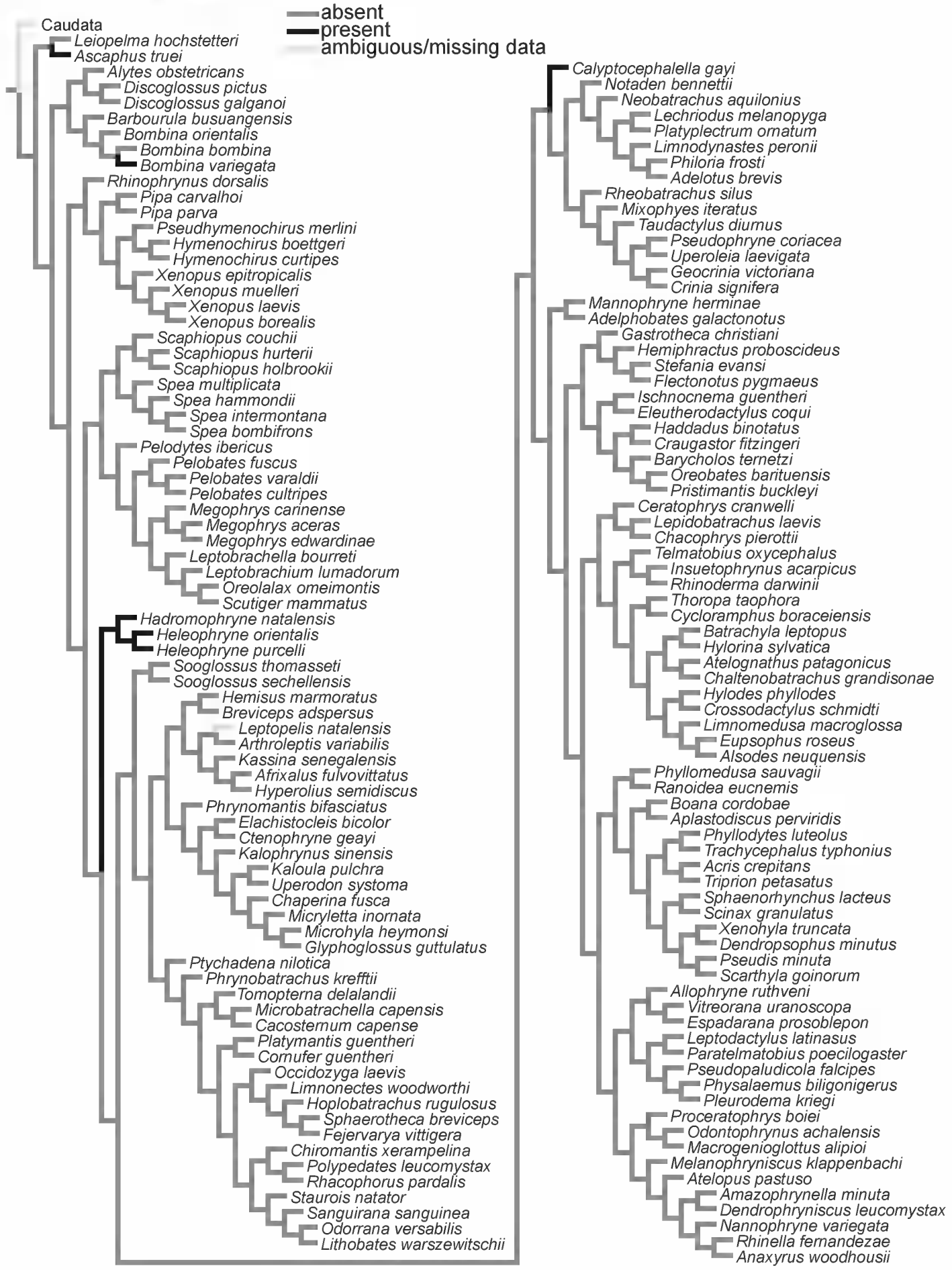


APPENDIX 5 continued

Character 3. M. Iumbricalis brevis indicis, origin from flexor plate

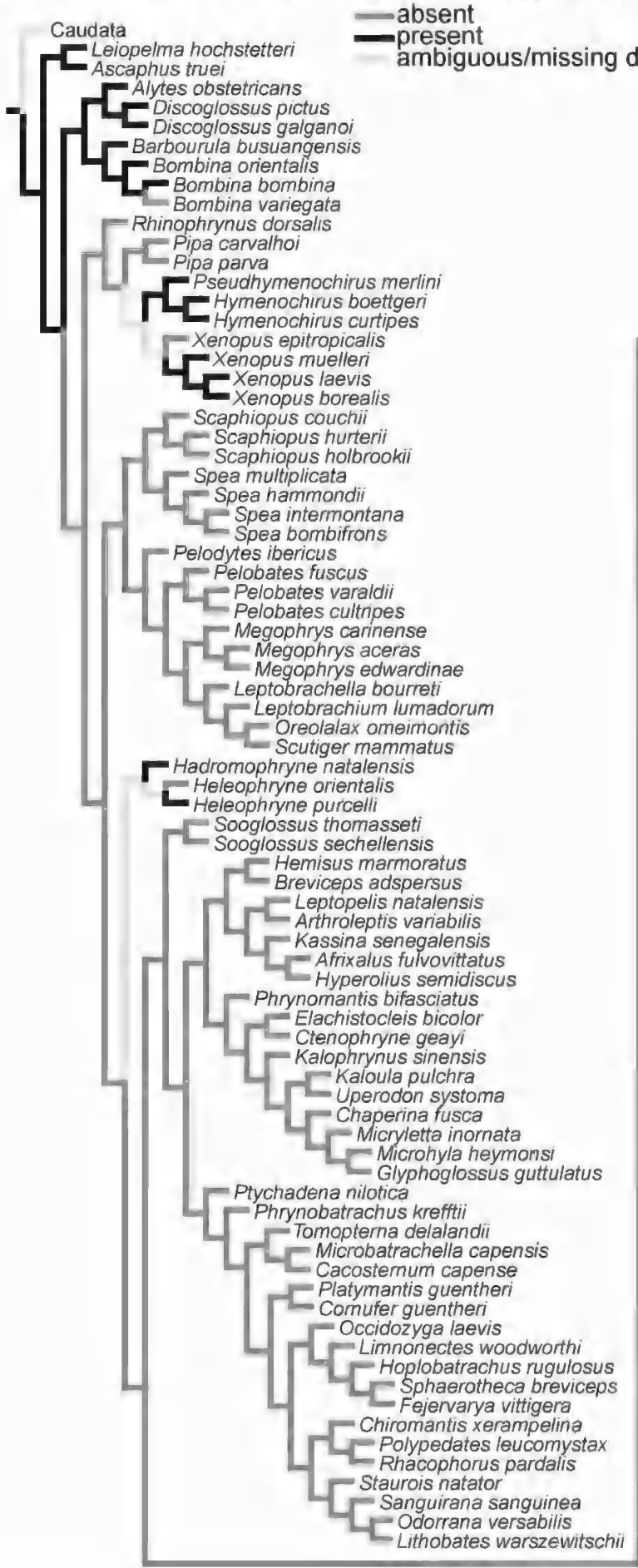

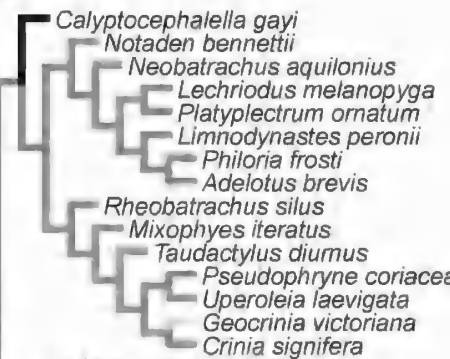

Mannophryne herminae

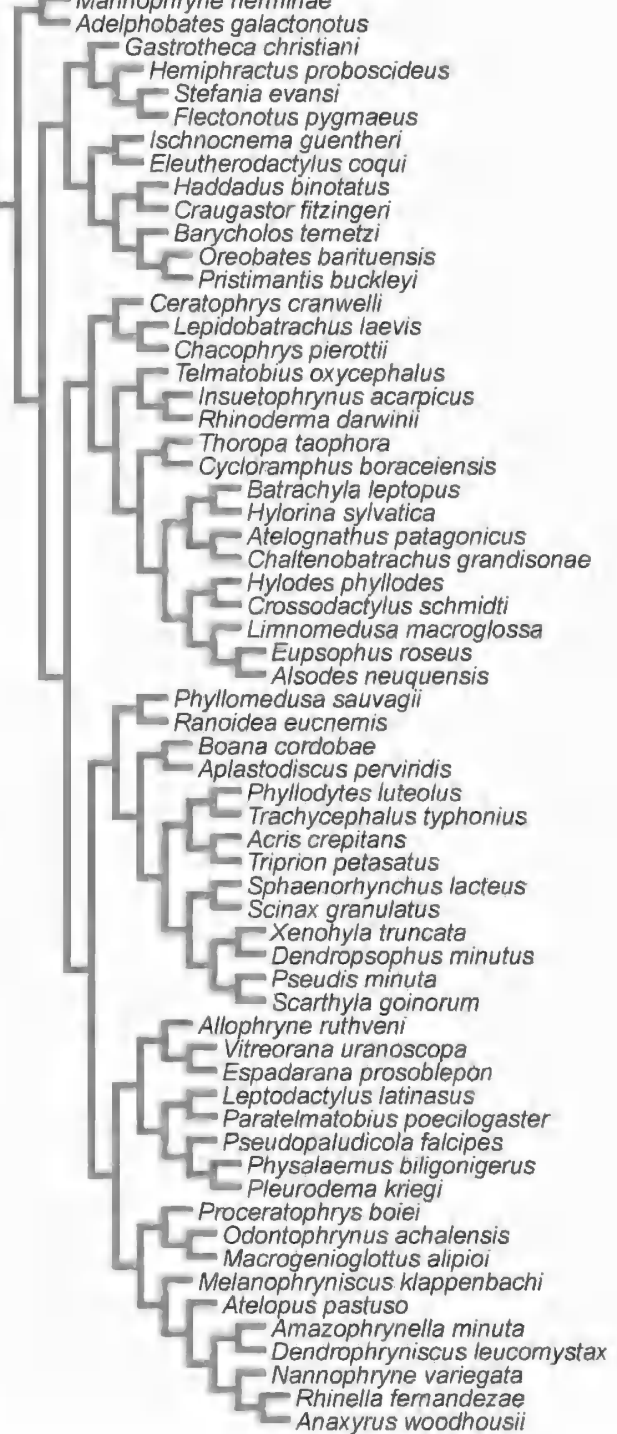


APPENDIX 5 continued

Character 4. M. lumbricalis brevis indicis, origin from distal carpals
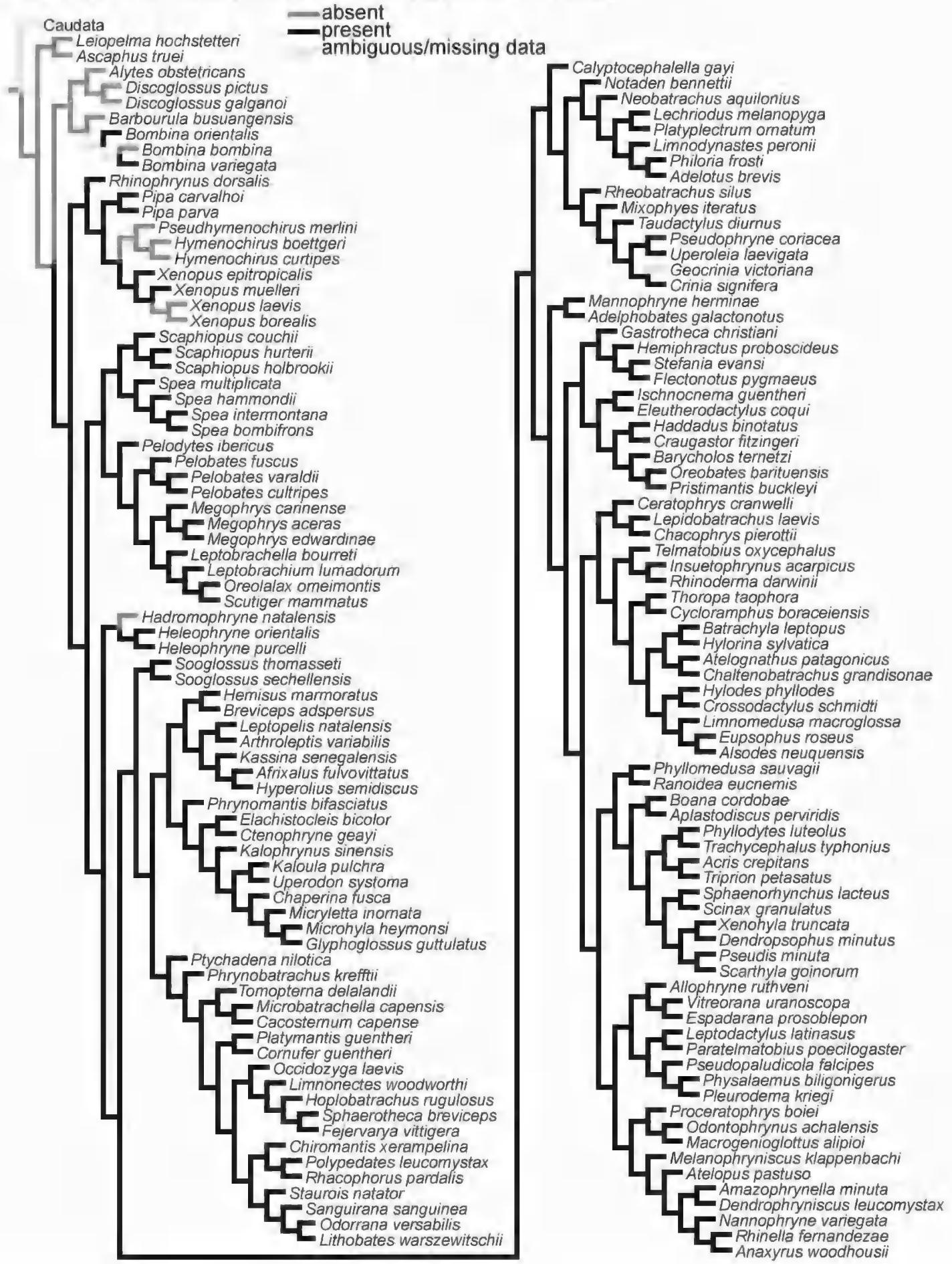
APPENDIX 5 continued

Character 5. M. lumbricalis brevis digiti III, slip from flexor plate and/or tendo superficialis digiti III

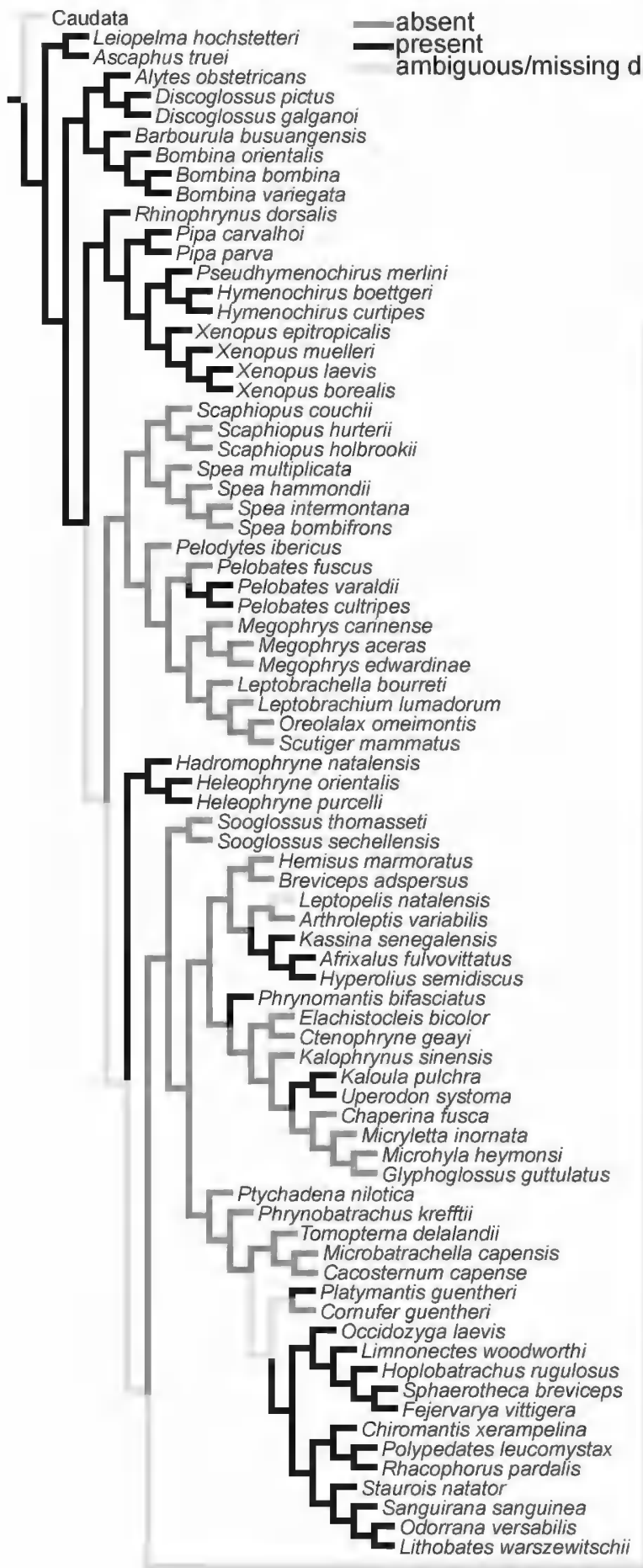

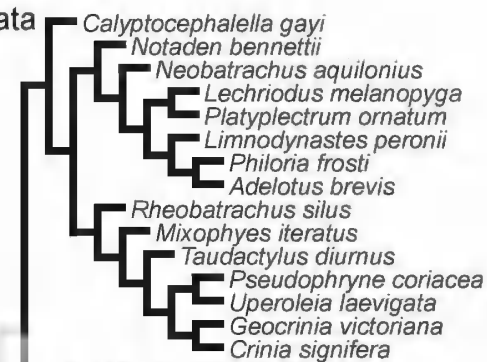

- Mannophryne herminae

- Adelphobates galactonotus - Gastrotheca christiani Hemiphractus proboscideus - Stefania evansi Flectonotus pygmaeus - Ischnocnema guenther Eleutherodactylus coqui Haddadus binotatus Craugastor fitzingen Barycholos temetzi - Oreobates barituensis - Pristimantis buckley - Ceratophrys cranwelli - Lepidobatrachus laevis - Chacophrys pierotti - Telmatobius oxycephalus

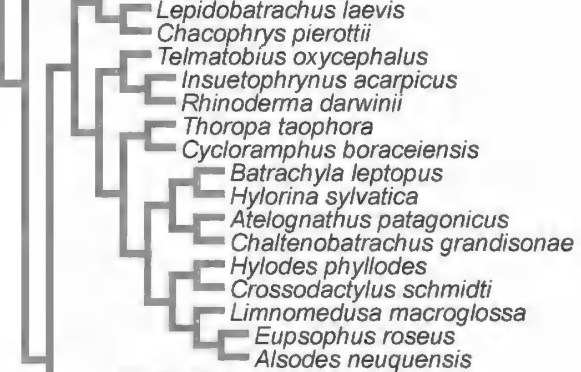
Phyllomedusa neuquensis

$$
\begin{gathered}
\text { Phyllomedusa sauvagii } \\
\text { Ranoidea eucnemis } \\
\text { Boana cordobae } \\
\text { Aplastodiscus perviridis } \\
\text { Phyllodytes luteolus } \\
\text { Trachycephalus typhonius } \\
\text { Acris crepitans } \\
\text { Triprion petasatus } \\
\text { Sphaenorhynchus lacteus } \\
\text { Scinax granulatus } \\
\text { Xenohyla truncata } \\
\text { Dendropsophus minutus } \\
\text { Pseudis minuta } \\
\text { Scarthyla goinorum }
\end{gathered}
$$
- Allophryne ruthveni

- Vitreorana uranoscopa - Espadarana prosoblepon - Leptodactylus latinasus - Paratelmatobius poecilogaster - Pseudopaludicola falcipes - Physalaemus biligonigerus Pleurodema krieg Proceratophrys boiei

- Odontophrymus achalensis Macrogenioglottus alipioi - Melanophryniscus klappenbachi Atelopus pastuso Atelopus pastuso - Dendrophryniscus leucomystax - Nannophryne variegata - Rhinella fermandezae - Anaxyrus woodhousii 
APPENDIX 5 continued

Character 6. M. lumbricalis brevis digiti III, slip from distal carpals

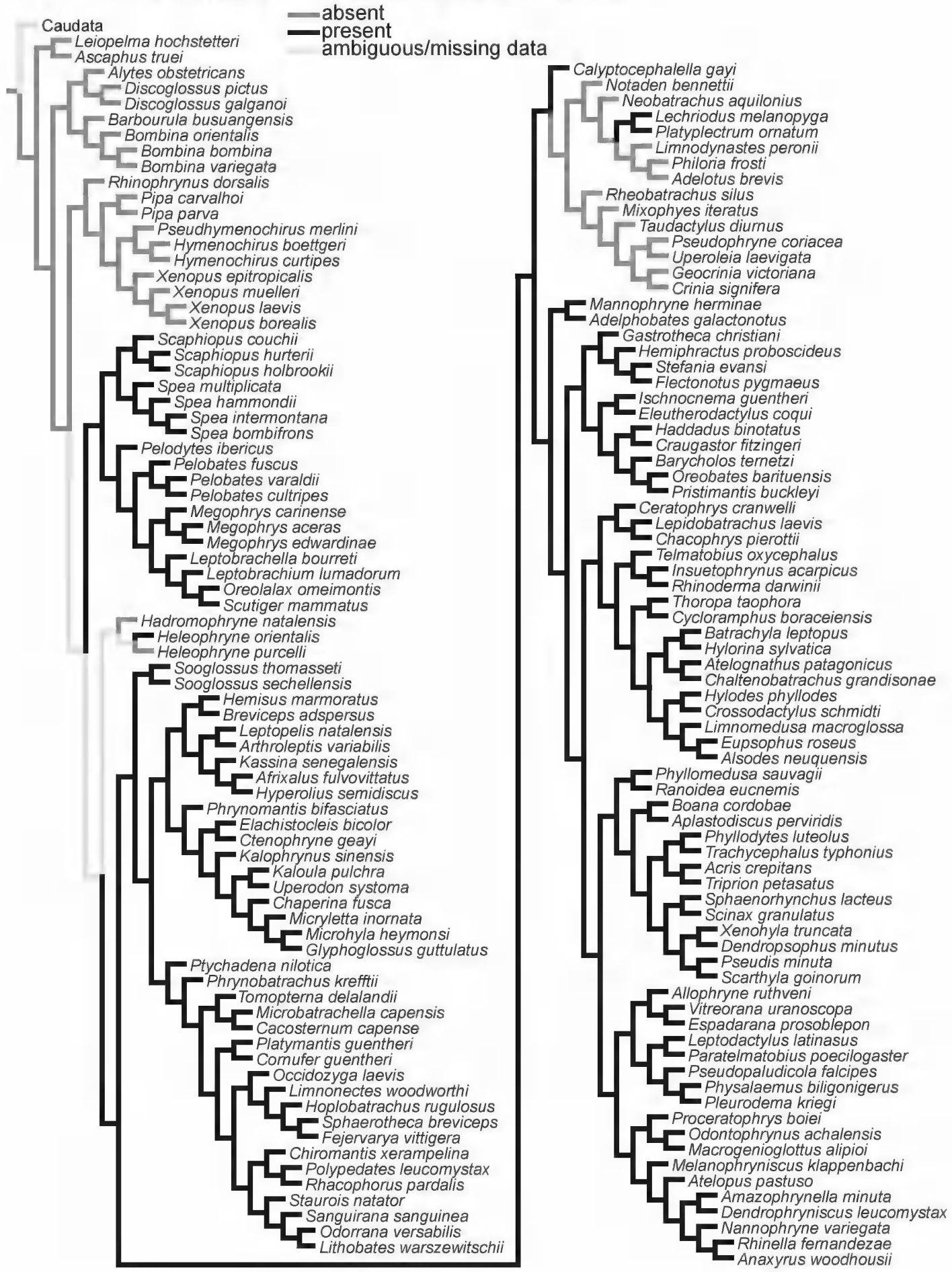


APPENDIX 5 continued

Character 7. Medial m. lumbricalis brevis digiti IV, supplementary medial slip from distal carpals

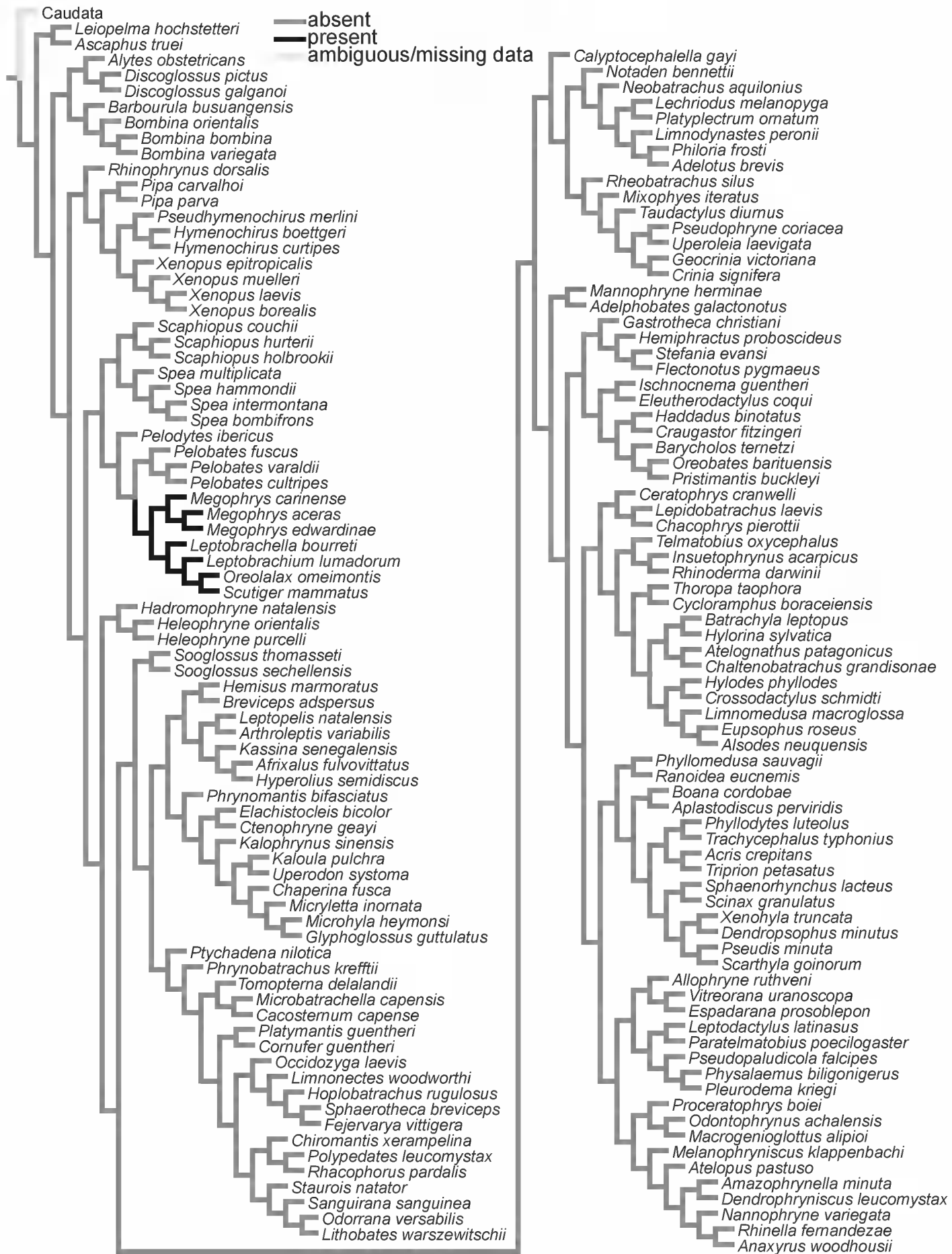


APPENDIX 5 continued

Character 8. M. flexor minimus indicis

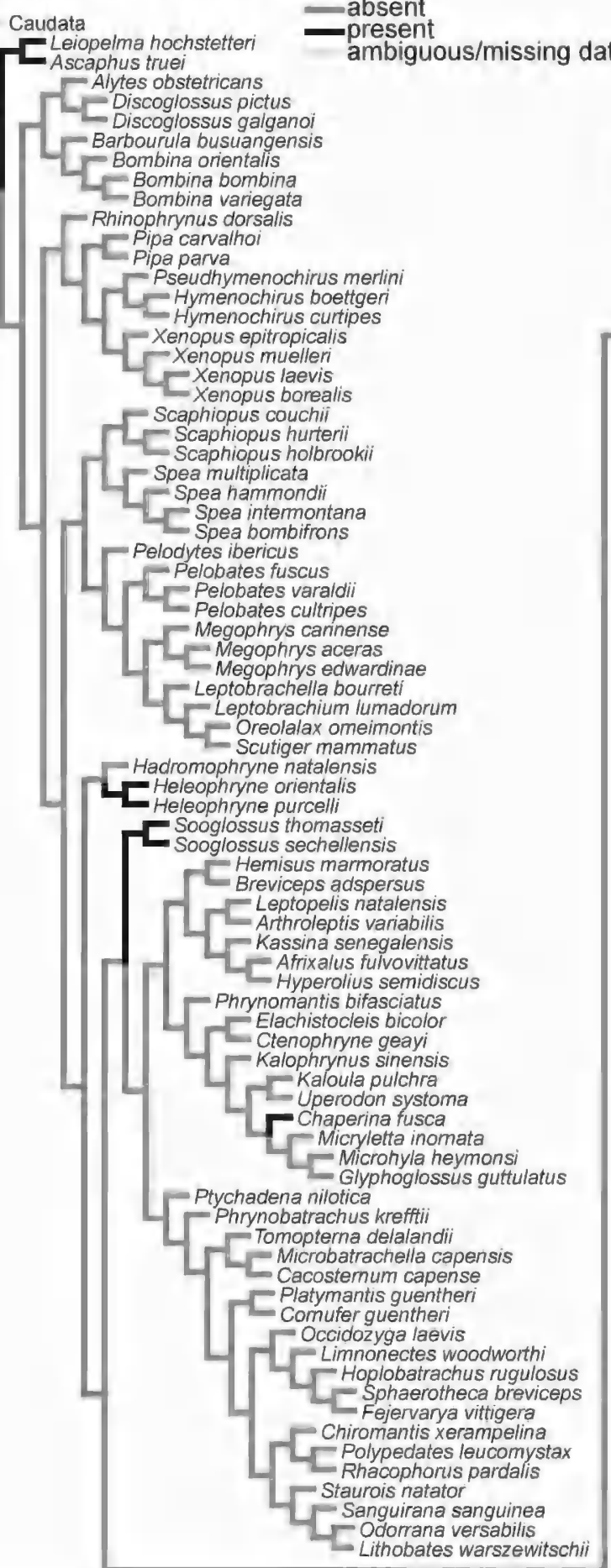

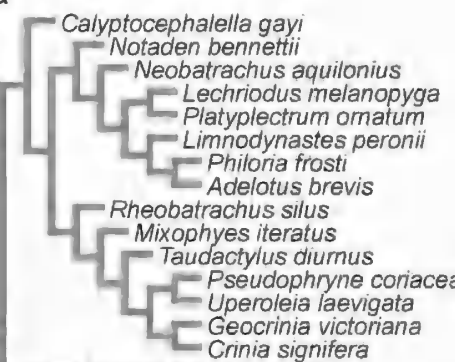

Mannophryne herminae

Adelphobates galactonotus - Gastrotheca christiani Hemiphractus proboscideus - Stefania evansi Flectonotus pygmaeus

- Ischnocnema guenther Eleutherodactylus coqui Haddadus binotatus

- Craugastor fitzingeri - Barycholos ternetzi - Oreobates barituensis - Pristimantis buckleyi - Ceratophrys cranwelli - Lepidobatrachus laevis - Chacophrys pierottii Telmatobius oxycephalus - Insuetophrynus acarpicus - Rhinoderma darwini - Thoropa taophora - Cycloramphus boraceiensis - Batrachyla leptopus

- Atelognathus patagonicus

- Chaltenobatrachus grandisonae - Hylodes phyllodes - Crossodactylus schmidti - Limnomedusa macrogiossa Eupsophus roseus

Phyllomedusa sauvagi - Ranoidea eucnemis

$$
\begin{aligned}
& \text { Boana cordobae } \\
& \text { Aplastodiscus perviridis } \\
& \text { Phyllodytes luteolus } \\
& \text { Trachycephalus typhonius } \\
& \text { Acris crepitans } \\
& \text { Tripnon petasatus } \\
& \text { Sphaenorhynchus lacteus } \\
& \text { Scinax granulatus } \\
& \text { Xenohyla truncata } \\
& \text { Dendropsophus minutus } \\
& \text { Pseudis minuta } \\
& \text { Scarthyla goinorum } \\
& \text { Allophryne ruthveni }
\end{aligned}
$$

- Vitreorana uranoscopa - Espadarana prosoblepon - Leptodactylus latinasus - Paratelmatobius poecilogaster - Pseudopaludicola falcipes - Physalaemus biligonigerus - Pleurodema krieg Proceratophrys boiei - Odontophrynus achalensis - Macrogenioglottus alipioi Melanophryniscus klappenbachi Atelopus pastuso

- Amazophrynella minuta Dendrophryniscus leucomystax Nannophryne variegata - Rhinella fernandezae Anaxyrus woodhousii 
APPENDIX 5 continued

Character 9. M. flexor minimus digiti IV (FMD IV), position of the origin with respect to the $\mathrm{m}$. intermetacarpalis II (IMC II) Caudata

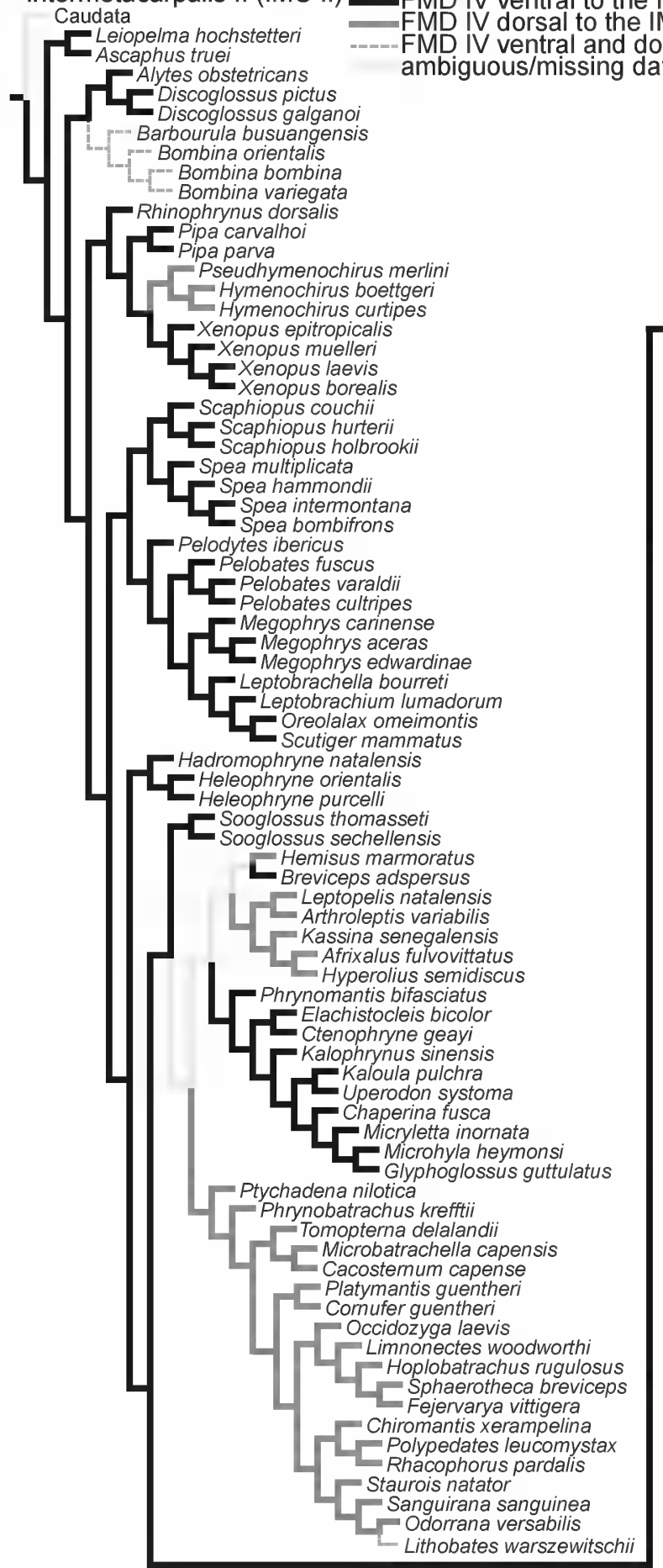

$$
\begin{aligned}
& \text { IMC II } \\
& \text { dorsal to the IMC II } \\
& \text { data - Calyptocephalell }
\end{aligned}
$$

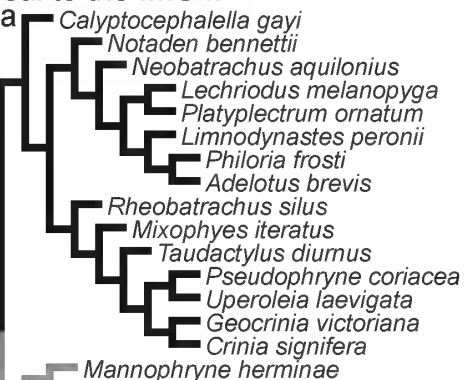

Mannophryne herminae

Adelphobates galactonotus Gastrotheca christiani Hemiphractus proboscideus - Stefania evansi Flectonotus pygmaeus

- Ischnocnema guentheri Eleutherodactylus coqui Haddadus binotatus Craugastor fitzingeri - Barycholos ternetzi --- Oreobates barituensis - Pristimantis buckleyi Ceratophrys cranwelli Chacophrys pierottii
Telmatobius oxycephalus
Insuetophrynus acarpicus
Rhinoderma danwinii
Thoropa taophora
Cycloramphus boraceiensis
Batrachyla leptopus Hylorina sylvatica

Atelognathus patagonicus

Chaltenobatrachus grandisonae Hylodes phyllodes Crossodactylus schmidti Limnomedusa macroglossa -Eupsophus roseus Alsodes neuquensis Phyllomedusa sauvagii

$$
\begin{gathered}
\text { Ranoidea eucnemis } \\
\text { Boana cordobae } \\
\text { Aplastodiscus perviridis } \\
\text { Phyllodytes luteolus } \\
\text { Trachycephalus typhonius } \\
\text { Acris crepitans } \\
\text { Triprion petasatus } \\
\text { Sphaenorhynchus lacteus } \\
\text { Scinax granulatus } \\
\text { Xenohyla truncata } \\
\text { Dendropsophus minutus } \\
\text { Pseudis minuta } \\
\text { Scarthyla goinorum }
\end{gathered}
$$
- Allophryne ruthveni - Vitreorana uranoscopa - Espadarana prosoblepon - Leptodactylus latinasus

- Paratelmatobius poecilogaster - Pseudopaludicola falcipes Pseudopaludicola falcipes
Physalaemus biligonigerus
Pleurodema kriegi Proceratophrys boiei

Odontophrynus achalensis - Macrogenioglottus alipioi - Melanophryniscus klappenbachi Atelopus pastuso

- Amazophrynella minuta

Dendrophryniscus leucomystax - Nannophryne variegata Rhinella fernandezae - Anaxyrus woodhousii 
APPENDIX 5 continued

Character 10. M. contrahentis caput longus distalis
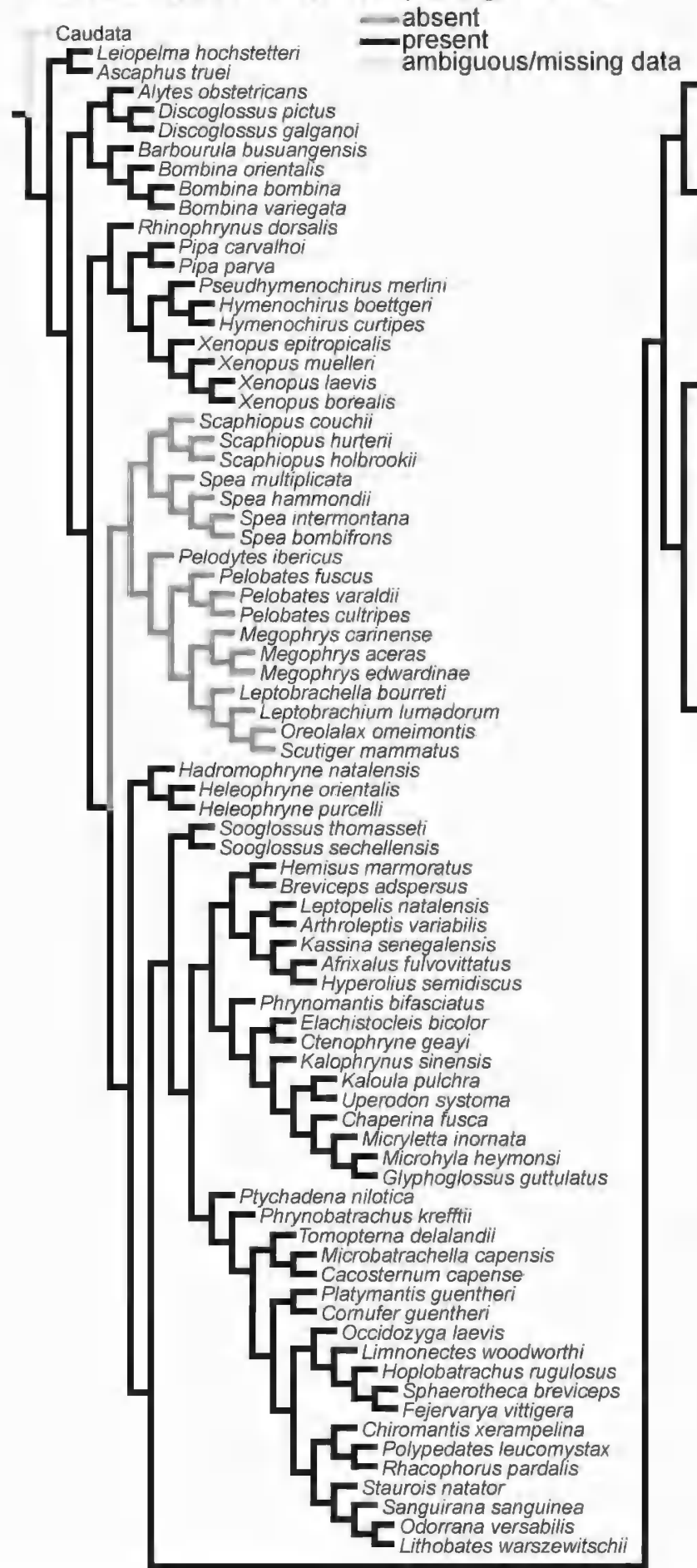

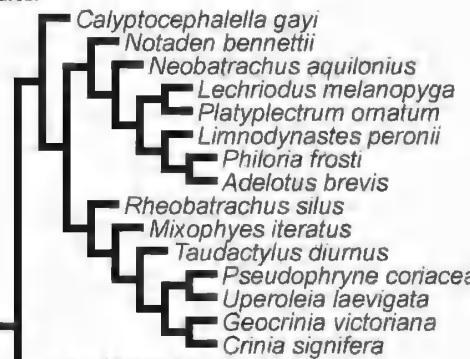

- Mannophryne herminae Adelphobates galactonotus - Gastrotheca christiani

Hemiphractus proboscideus
Stefania evansi
Flectonotus pygmaeus
Ischnocnema guentheri
Eleutherodactylus coqui
Haddadus binotatus
Craugastor fitzingeri
Barycholos ternetzi
Oreobates barituensis
Pristimantis buckleyi
Ceratophrys cranwelli
Lepidobatrachus laevis
Chacophrys pierottii
Telmatobius oxycephalus
Insuetophrynus acarpicus
Rhinoderma darwinii
Thoropa taophora
Cycloramphus boraceiensis
Batrachyla leptopus
Hylorina sylvatica
Atelognathus patagonicus
Chaltenobatrachus grandisonae
Hylodes phyllodes
Crossodactylus schmidti
Limnomedusa macrogiossa
E Eupsophus roseus
Elsodes neuquensis
- Phyllomedusa saluaquensis

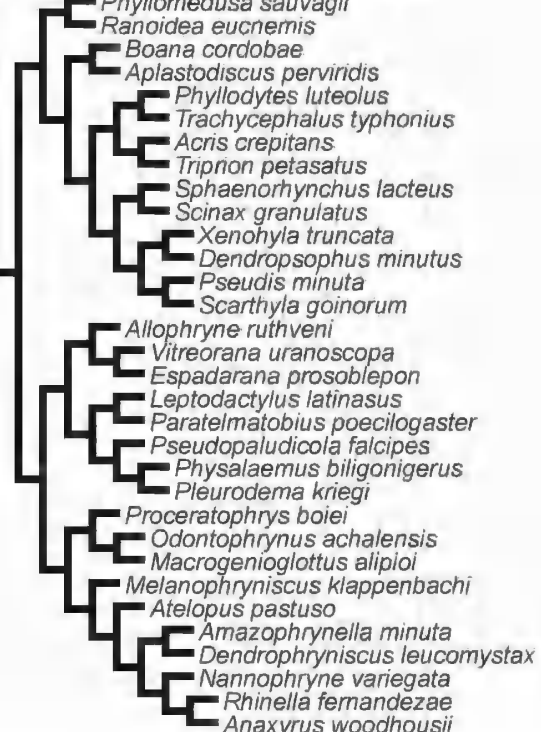


APPENDIX 5 continued

Character 11. M. extensor brevis medius digiti III, slip from the mediodistal end of radioulna

$$
\begin{array}{ll}
\text { Caudata } & \text { absent } \\
\text { Leiopelma hochstelteri } & \text { present } \\
\text { Ascaphus truei } & \text { ambiguous/missing data }
\end{array}
$$

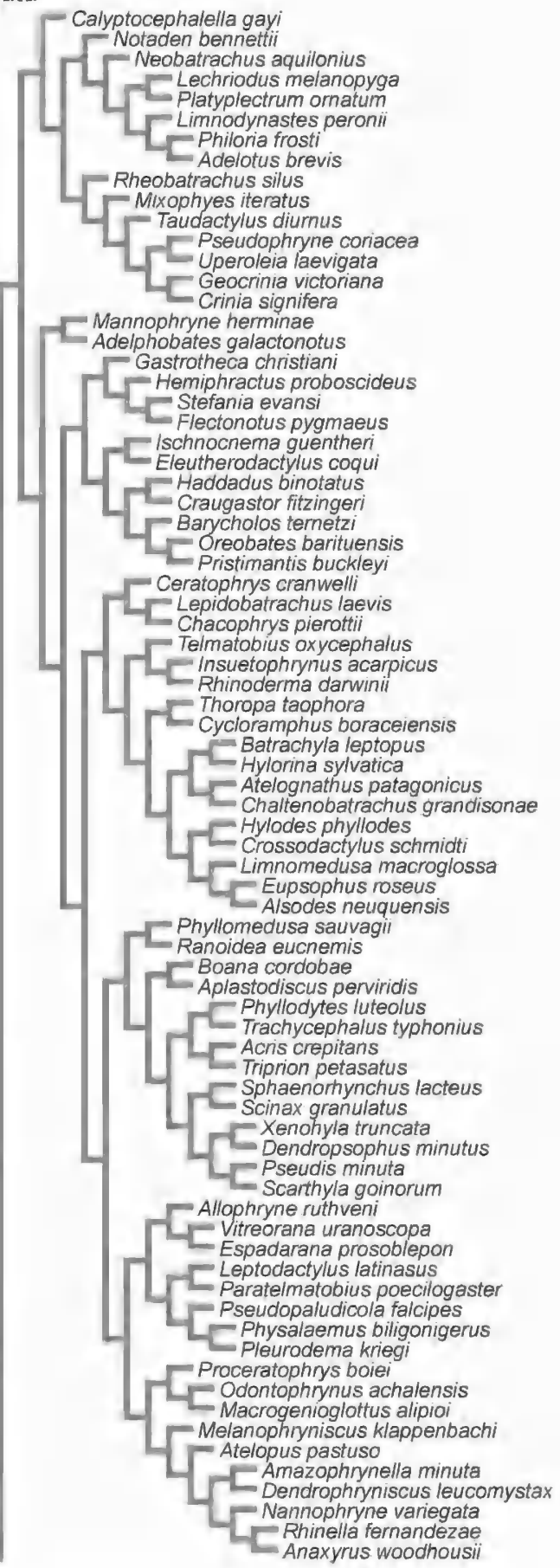


APPENDIX 5 continued

Character 12. M. extensor brevis medius digiti IV, origin from the radiale

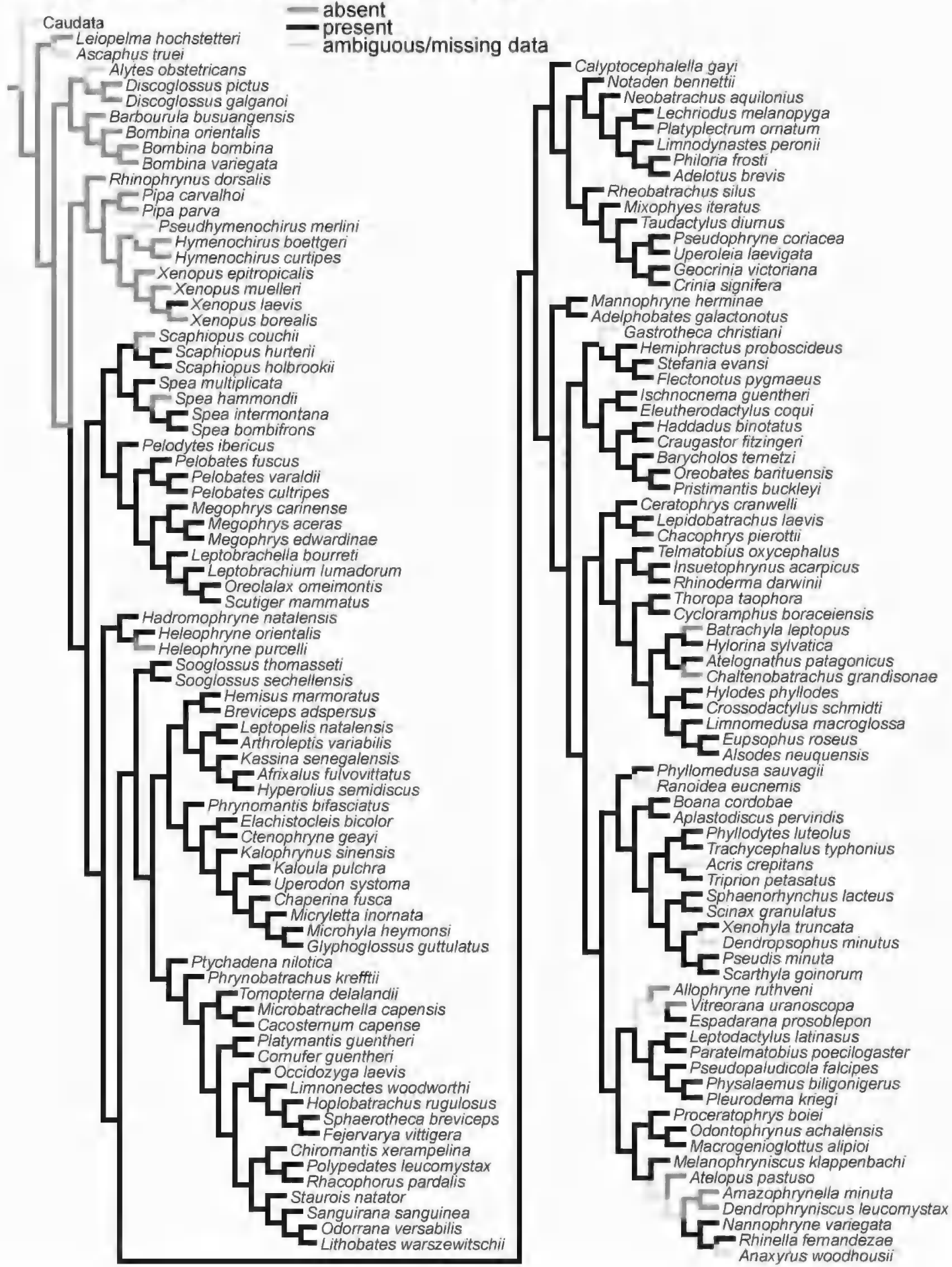


APPENDIX 5 continued

Character 13. Medial $\mathrm{m}$. dorsometacarpalis proximalis digiti IV, slip from metacarpal III

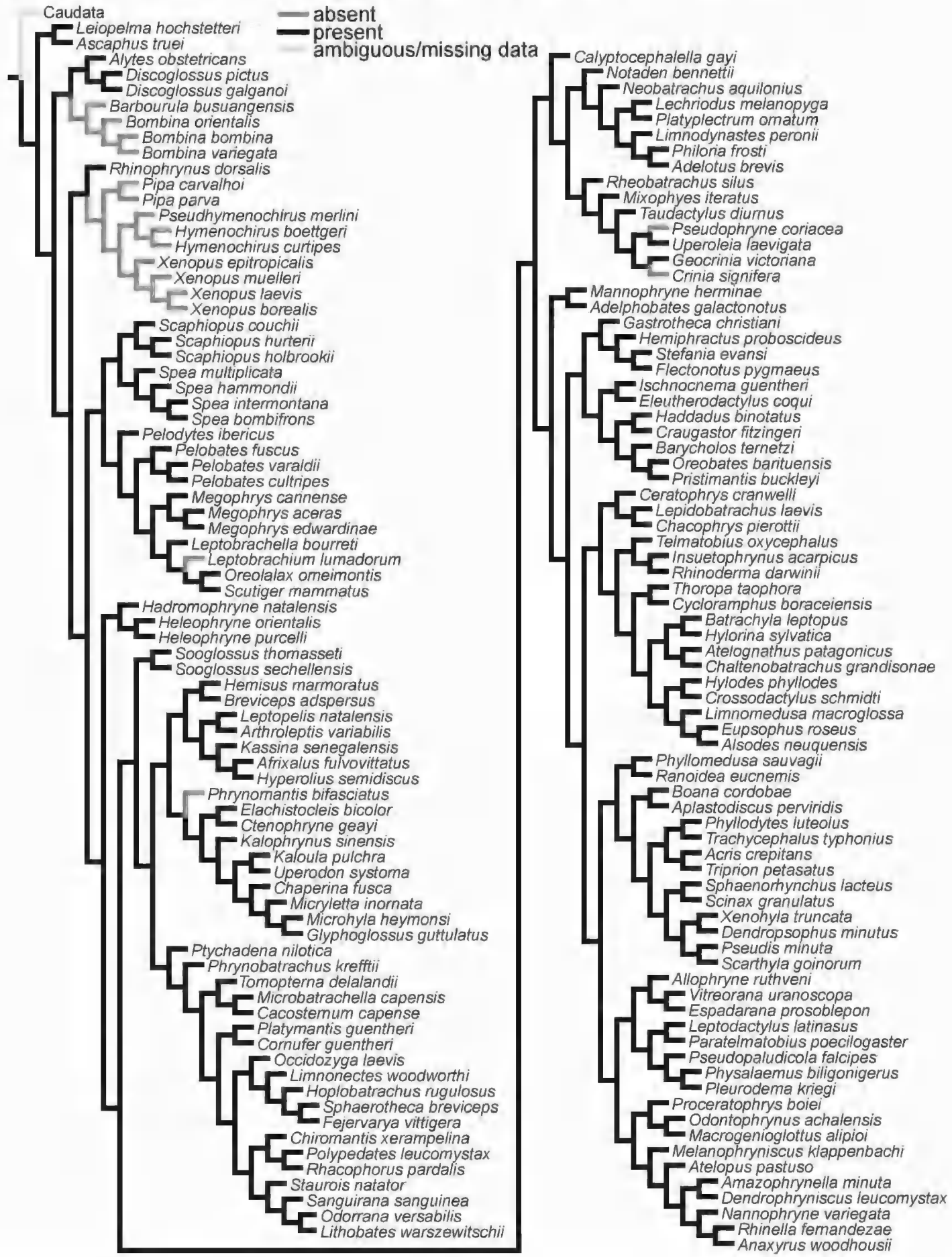


APPENDIX 5 continued

Character 14. M. lumbricalis brevis hallucis

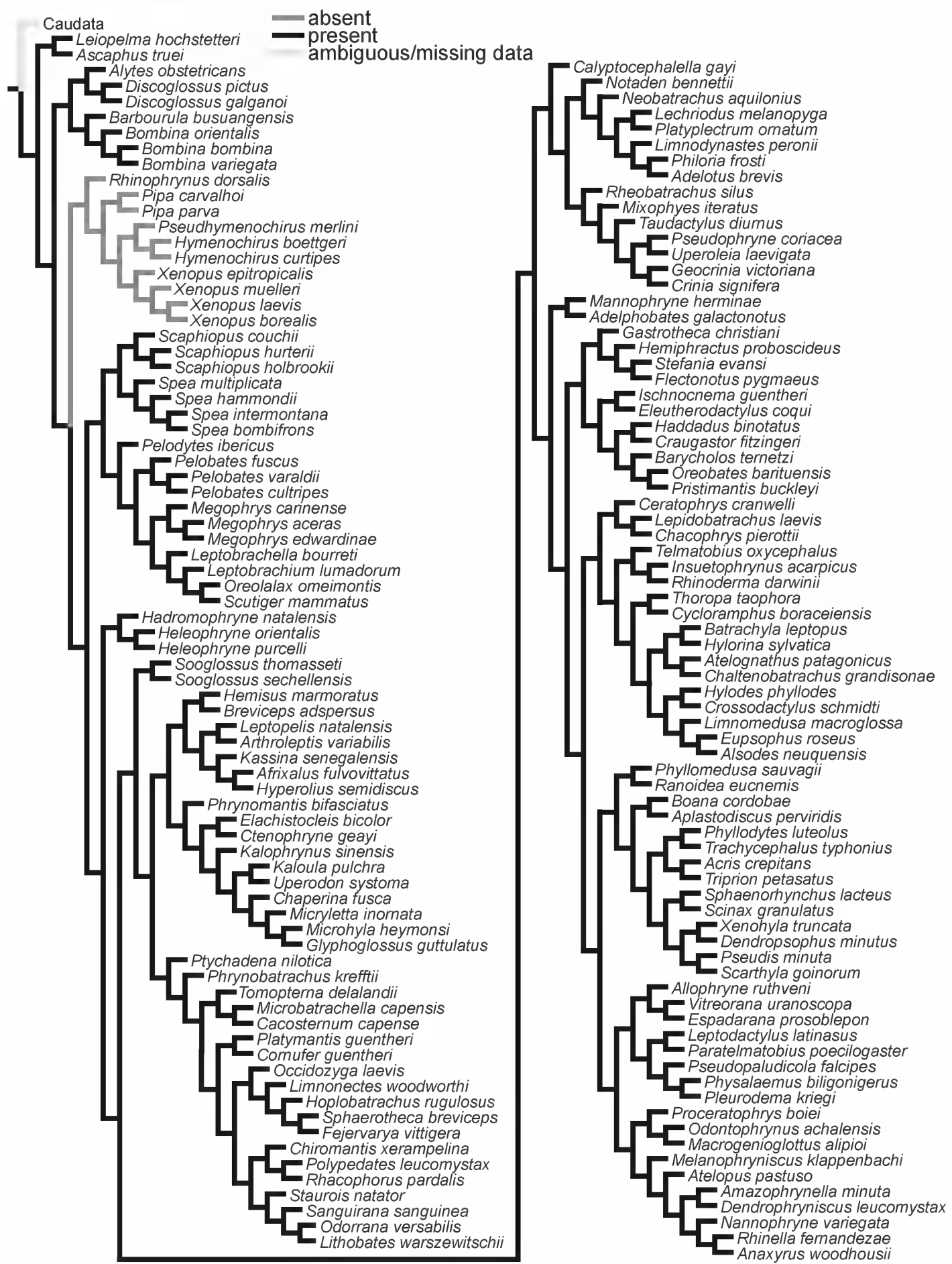


APPENDIX 5 continued

Character 15. Lateral $\mathrm{m}$. Iumbricalis brevis digiti IV, supplementary origin from the proximal end of metatarsal IV

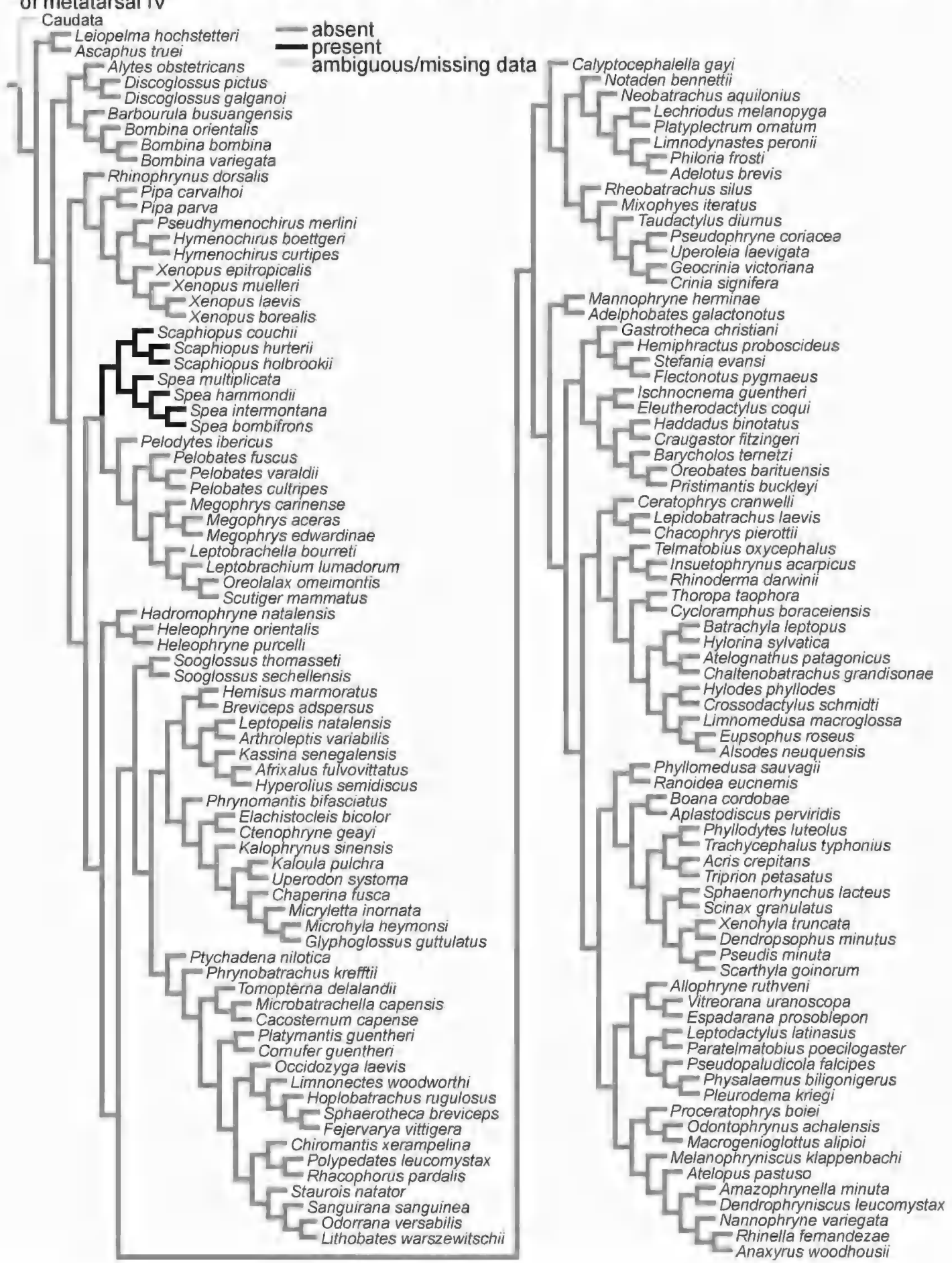


APPENDIX 5 continued

Character 16. M. contrahentis pedis digiti V

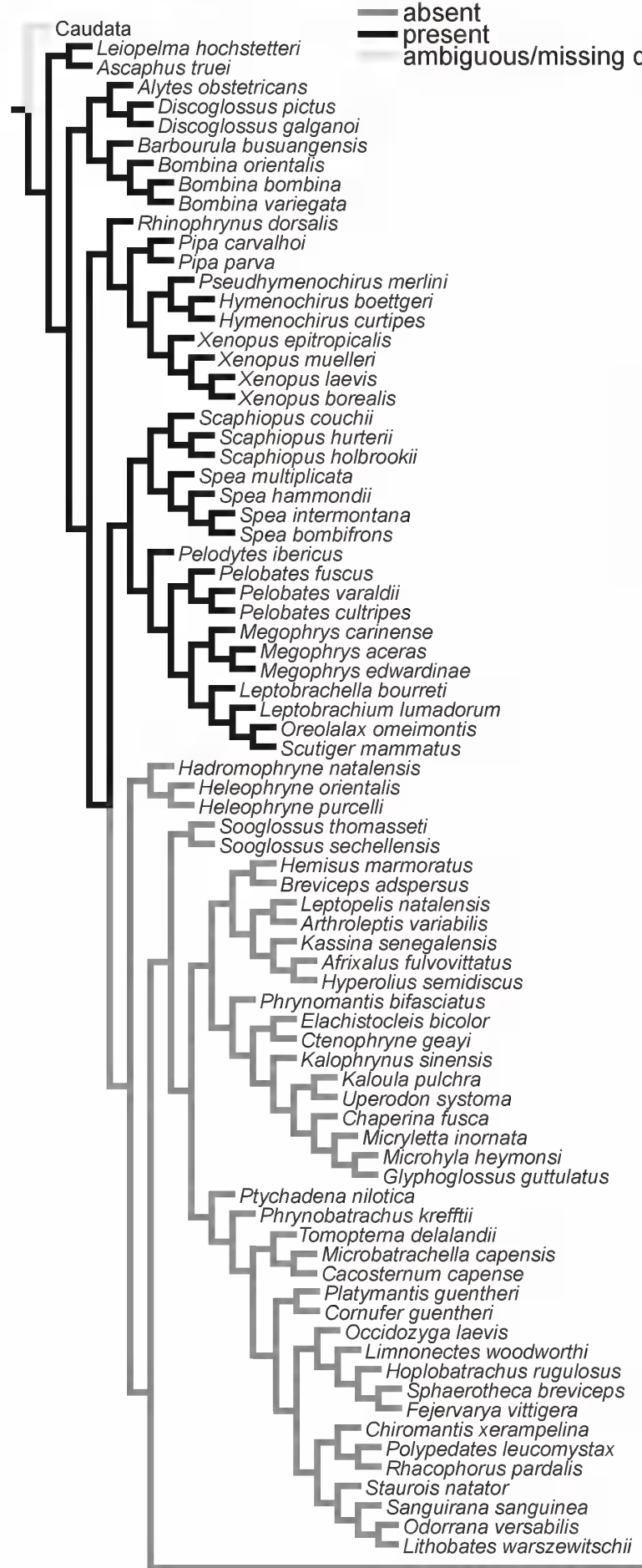

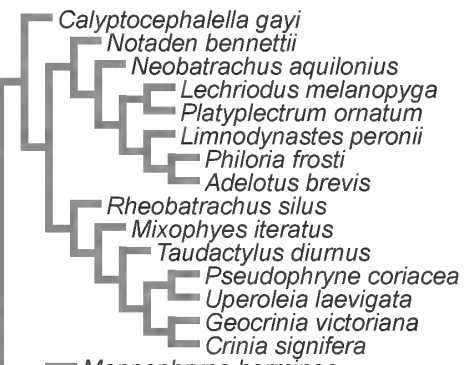

Mannophryne herminae

Adelphobates galactonotus - Gastrotheca christiani - Hemiphractus proboscideus - Stefania evansi Flectonotus pygmaeus

- Ischnocnema guenther

Eleutherodactylus coqui Haddadus binotatus

Craugastor fitzingeri - Barycholos ternetzi - Oreobates barituensis - Ceratophrys cranwelli Lepidobatrachus laevis - Chacophrys pierottii - Telmatobius oxycephalus Insuetophrynus acarpicus - Rhinoderma darwini - Thoropa taophora - Cycloramphus boraceiensis Batrachyla leptopus Hylorina sylvatica Atelognathus patagonicus - Chaltenobatrachus grandisonae Hylodes phyllodes - Crossodactylus schmidti - Limnomedusa macroglossa Eupsophus roseus Alsodes neuquensis - Phyllomedusa sauvagii Ranoidea eucnemis - Boana cordobae

Aplastodiscus perviridis Phyllodytes luteolus Trachycephalus typhonius - Acris crepitans

- Triprion petasatus

- Sphaenorhynchus lacteus Scinax granulatus Xenohyla truncata Dendropsophus minutus - Pseudis minuta Scarthyla goinorum - Allophryne ruthveni Vitreorana uranoscopa - Espadarana prosoblepon - Leptodactylus latinasus - Paratelmatobius poecilogaster - Pseudopaludicola falcipes - Physalaemus biligonigerus Pleurodema kriegi - Proceratophrys boiei Odontophrynus achalensis Macrogenioglottus alipioi - Melanophryniscus klappenbachi - Atelopus pastuso Amazophrynella minuta Dendrophryniscus leucomystax - Nannophryne variegata Rhinella fernandezae Anaxyrus woodhousii 
APPENDIX 5 continued

Character 17. M. extensor brevis superficialis digiti III

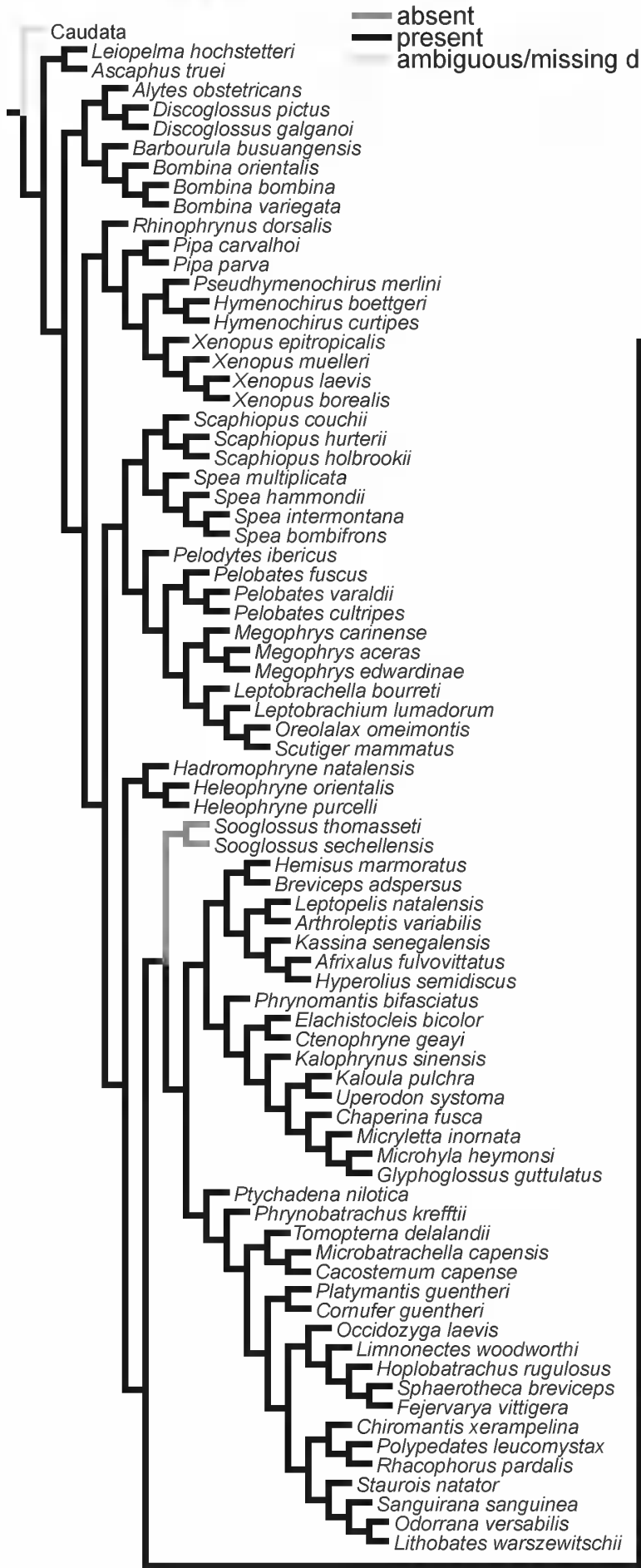

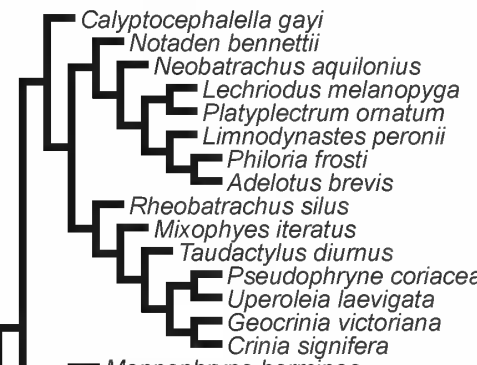

- Mannophryne herminae

드 Adelphobates galactonotus - Gastrotheca christiani

Hemiphractus proboscideus - Stefania evansi Flectonotus pygmaeus

Ischnocnema guentheri

Haddadus binotatus

Craugastor fitzingeri
Barycholos ternetzi

Oreobates barituensis

- Pristimantis buckley

Ceratophrys cranwelli

Chacophrys pierottii
Telmatobius oxycephalus

4 Insuetophrynus acarpicus

- Thoropa taophora

C Cycloramphus boraceiensis

Batrachyla leptopus

- Hylorina sylvatica

T-Atelognathus patagonicus

L Chaltenobatrachus grandisonae

- Hylodes phyllodes

- Crossodactylus schmidti

- Limnomedusa macroglossa

Eupsophus roseus

Alsodes neuquensis

- Phyllomedusa sauvagii

Lr Ranoidea eucnemis

Boana cordobae

- Aplastodiscus perviridis

Phyllodytes luteolus

- Acris crepitans

- Sphaenorhynchus lacteus

- Scinax granulatus

Xenohyla truncata

- Dendropsophus minutus

- Pseudis minuta

Scarthyla goinorum

- Allophryne ruthven

- Vitreorana uranoscopa

- Espadarana prosoblepon

- Leptodactylus latinasus

- Paratelmatobius poecilogaster

- Pseudopaludicola falcipes

Physalaemus biligonigerus

- Pleurodema kriegi

- Proceratophrys boiei

LEdontophrynus achalensis

Macrogenioglottus alipioi

- Melanophryniscus klappenbachi

- Atelopus pastuso

Amazophrynella minuta

Dendrophryniscus leucomystax

- Nannophryne variegata

Rhinella fernandezae

Anaxyrus woodhousi 
APPENDIX 5 continued

\section{Character 18. M. extensor brevis medius digiti IV

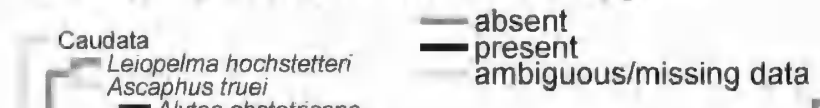

Ascaphus truei ambiguous/missing data

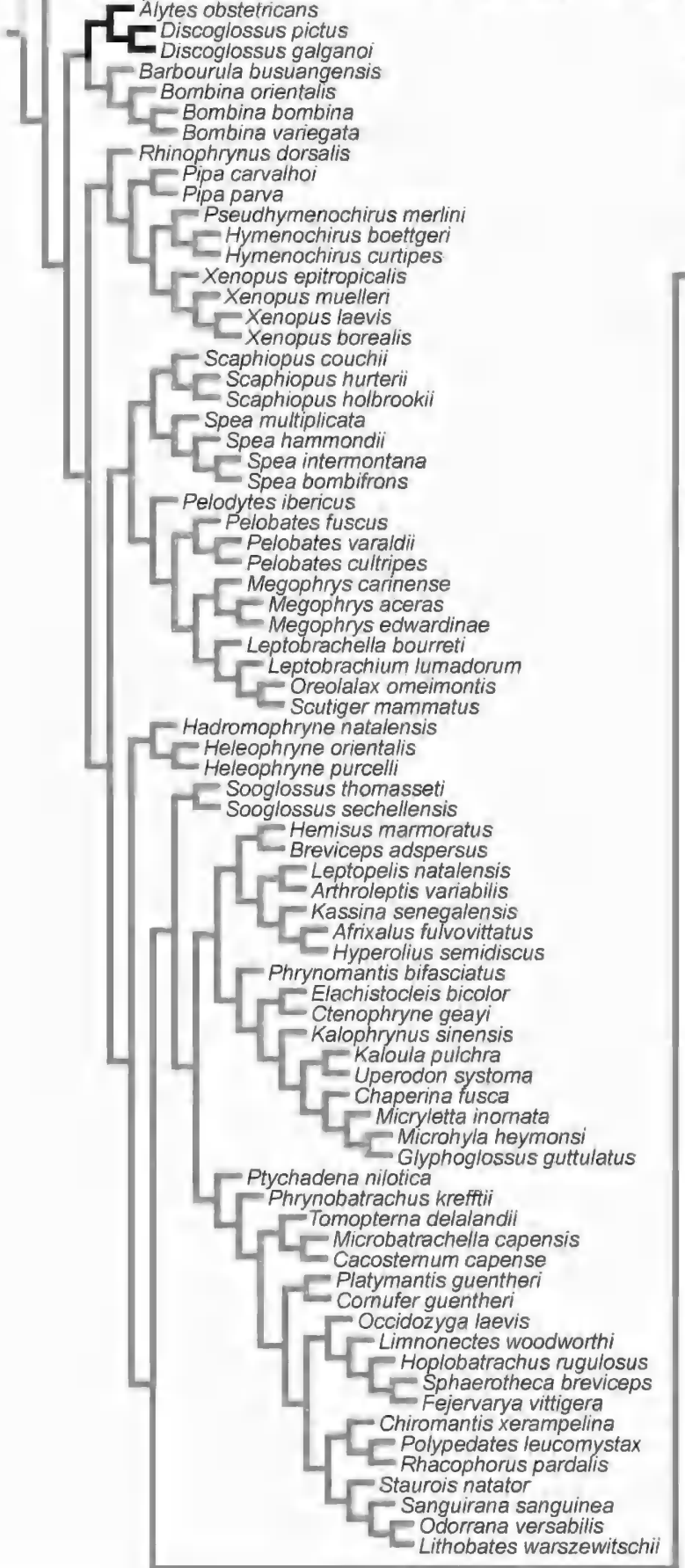

- Calyptocephalella gayi Notaden bennettii Neobatrachus aquilonius - Lechriodus melanopyga - Limnodynastes peroni CPhiloria frosti - Adelotus brevis

Rheobatrachus silus Mixophyes iteratus - Taudactylus diurnus - Pseudophryne coriacea - Uperoleia laevigata - Geocrinia victoriana Crinia signifera

Mannophryne herminae Adelphobates galactonotus - Gastrotheca christiani - Hemiphractus proboscideus - Stefania evansi Flectonotus pygmaeus - Ischnocnema quentheri Eleutherodactylus coqui - Haddadus binotatus - Barycholos ternetzi L Oreobates barituensis - Pristimantis buckley - Ceratophrys cranwelli Lepidobatrachus laevis Chacophrys pierottii
Telmatobius oxycephalus
Insuetophrynus acarpicus Chacophrys pierottii
Telmatobius oxycephalus
- Insuetophrynus acarpicus - Rhinoderma darwini Thoropa taophora
Cycloramphus boraceiensis
Batrachyla leptopus Hylorina sylvatica

Latelognathus patagonicus

- Chaltenobatrachus grandisonae Hylodes phyllodes - Crossodactylus schmidti - Limnomedusa macroglossa Eupsophus roseus - Alsodes neuquensis

- Phyllomedusa sauvagii Ranoidea eucnemis

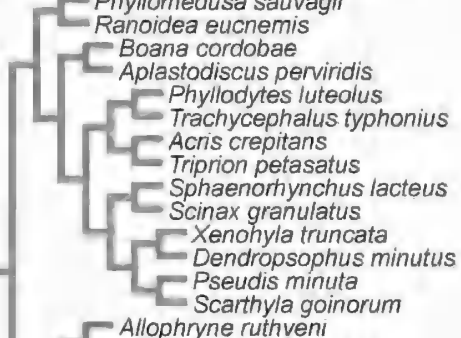
- Allophryne ruthveni - Vitreorana uranoscopa - Espadarana prosoblepon - Leptodactylus latinasus - Paratelmatobius poecilogaster - Pseudopaludicola falcipes Physalaemus biligonigerus - Pleurodema kriegi - Proceratophrys boiei - Odontophrynus achalensis - Macrogenioglottus alipioi - Melanophryniscus klappenbachi - Atelopus pastuso Amazophrynella minuta - Dendrophryniscus leucomystax - Nannophryne variegata - Rhinella femandeza Anaxyrus woodhousi 
APPENDIX 5 continued

Character 19. M. abductor brevis dorsalis digiti II

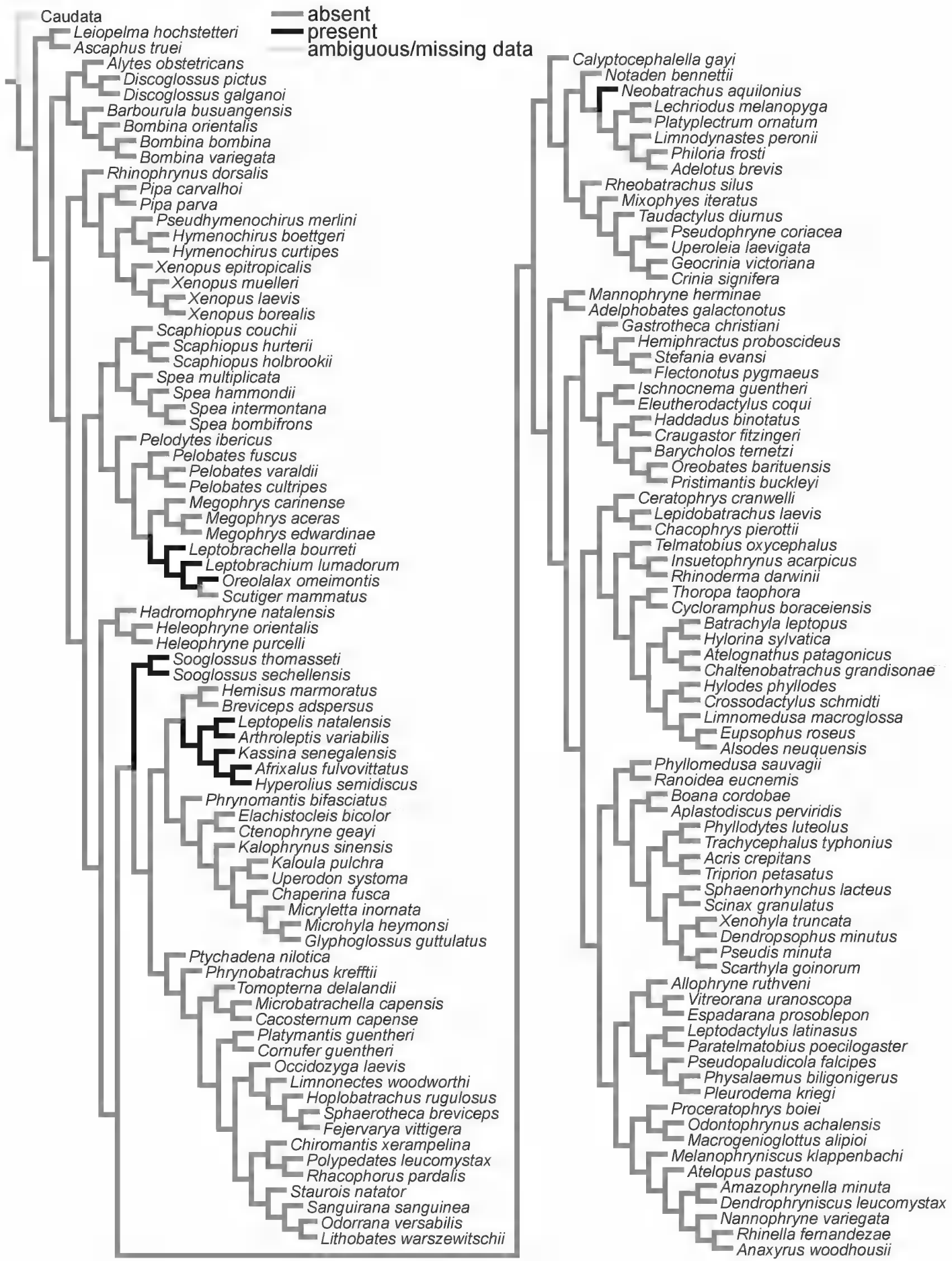


APPENDIX 5 continued

Character 20. M. abductor brevis dorsalis digiti III

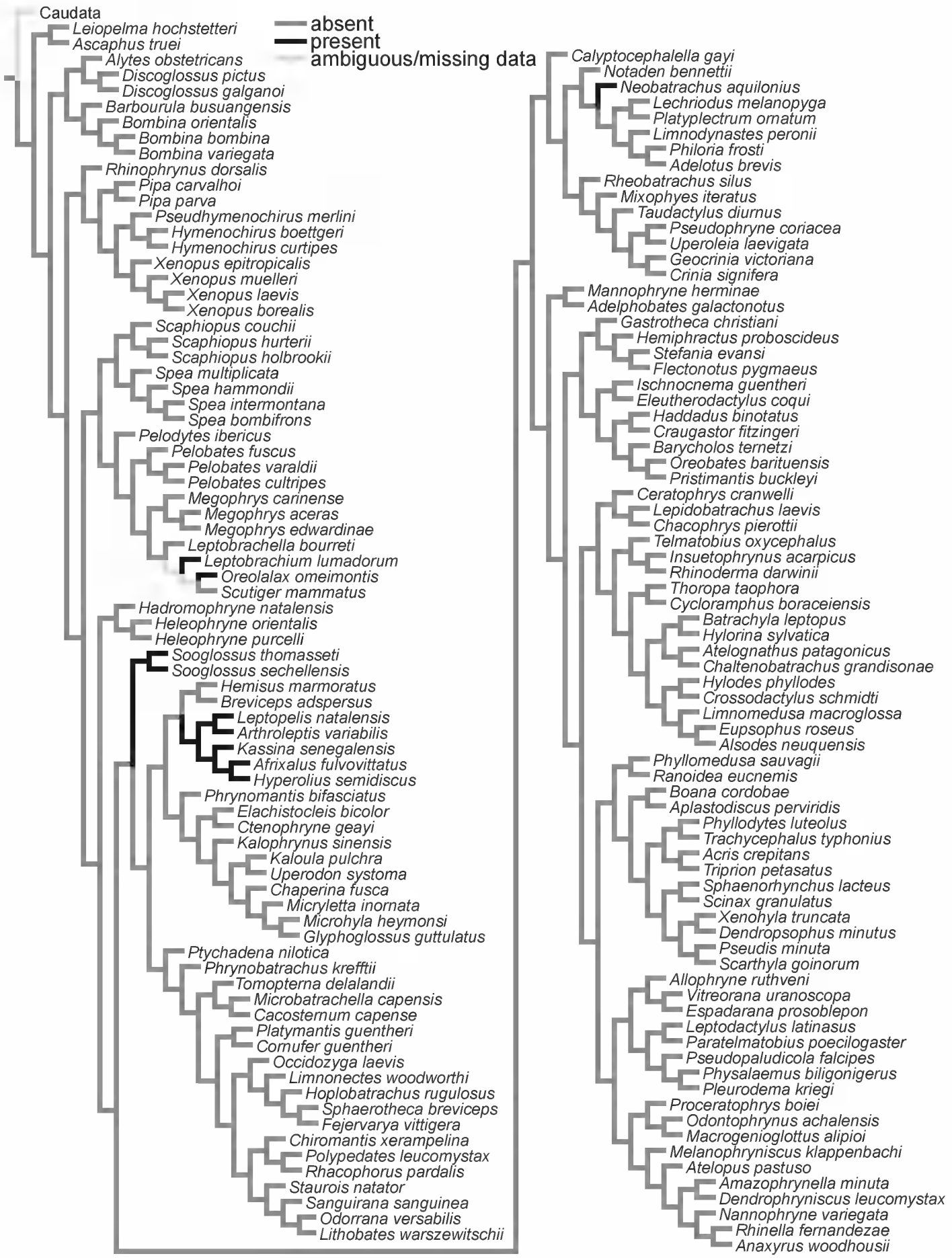




\section{APPENDIX 6}

\section{AbBreviations}

$\begin{array}{ll}\text { abd } & \text { abductor } \\ \text { DC } & \text { distal carpal } \\ \text { DT } & \text { distal tarsal } \\ \text { FI } & \text { fibulare } \\ \text { flex(s) } & \text { flexor(es }) \\ \text { M } & \text { metacarpal/metatarsal } \\ \text { PH } & \text { prehallux } \\ \text { PP } & \text { prepollex } \\ \text { RA } & \text { radiale } \\ \text { RU } & \text { radioulna } \\ \text { TI } & \text { tibiale } \\ \text { TI-FI } & \text { distal fused ends of tibiale and fibulare } \\ \text { UL } & \text { ulnare } \\ \text { Y } & \text { element } Y\end{array}$




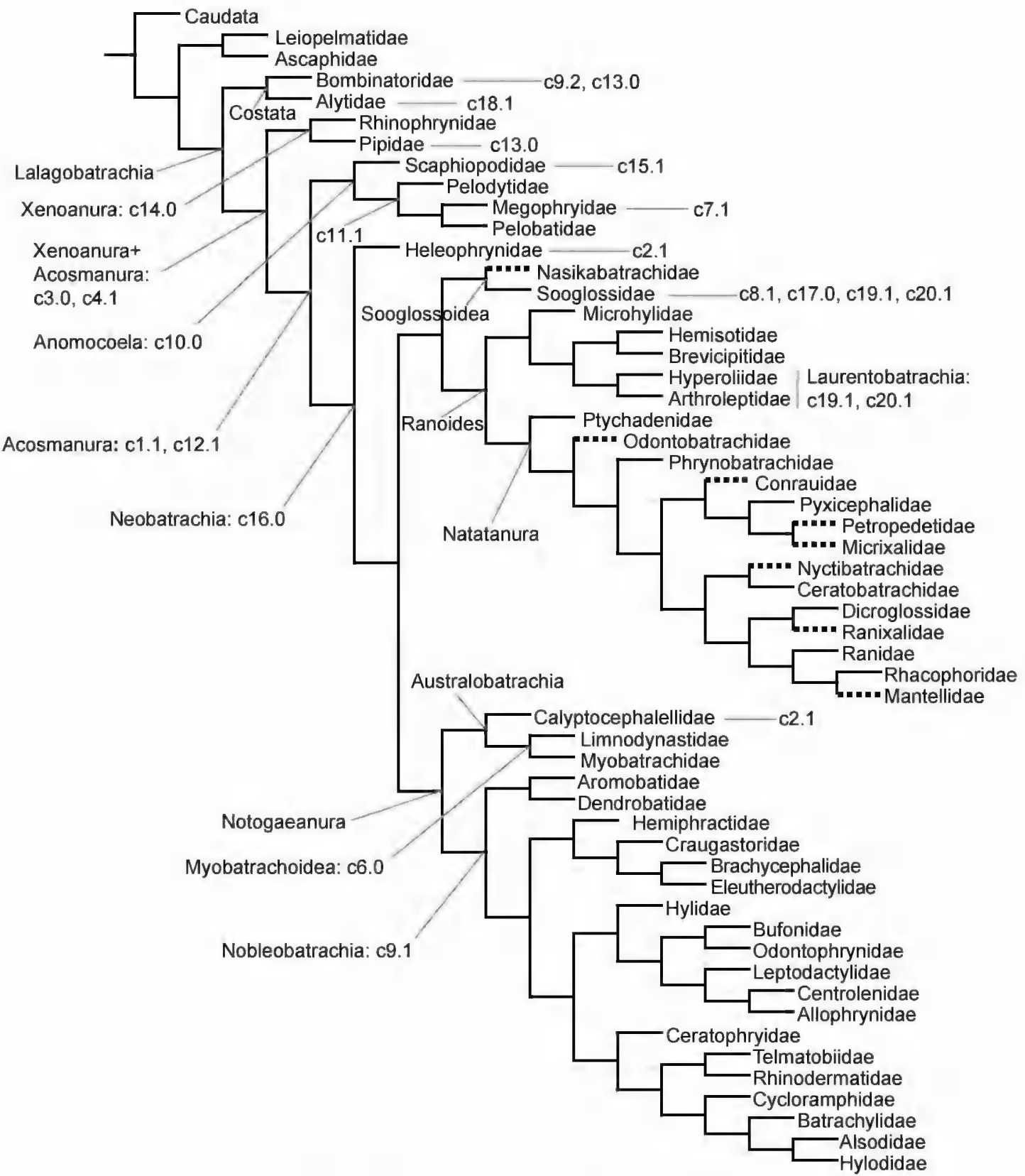

PLATE 1. Reduced tree at the family level following the topology of Jetz and Pyron (2018). The proposed synapomorphies, as obtained with the ancestral character state reconstruction (shown in appendix 5), are indicated in each node. Families represented by dotted lines in the phylogeny were not sampled in this study. Major clades with no newly suggested synapomorphies but addressed in Discussion are also indicated for guide. 

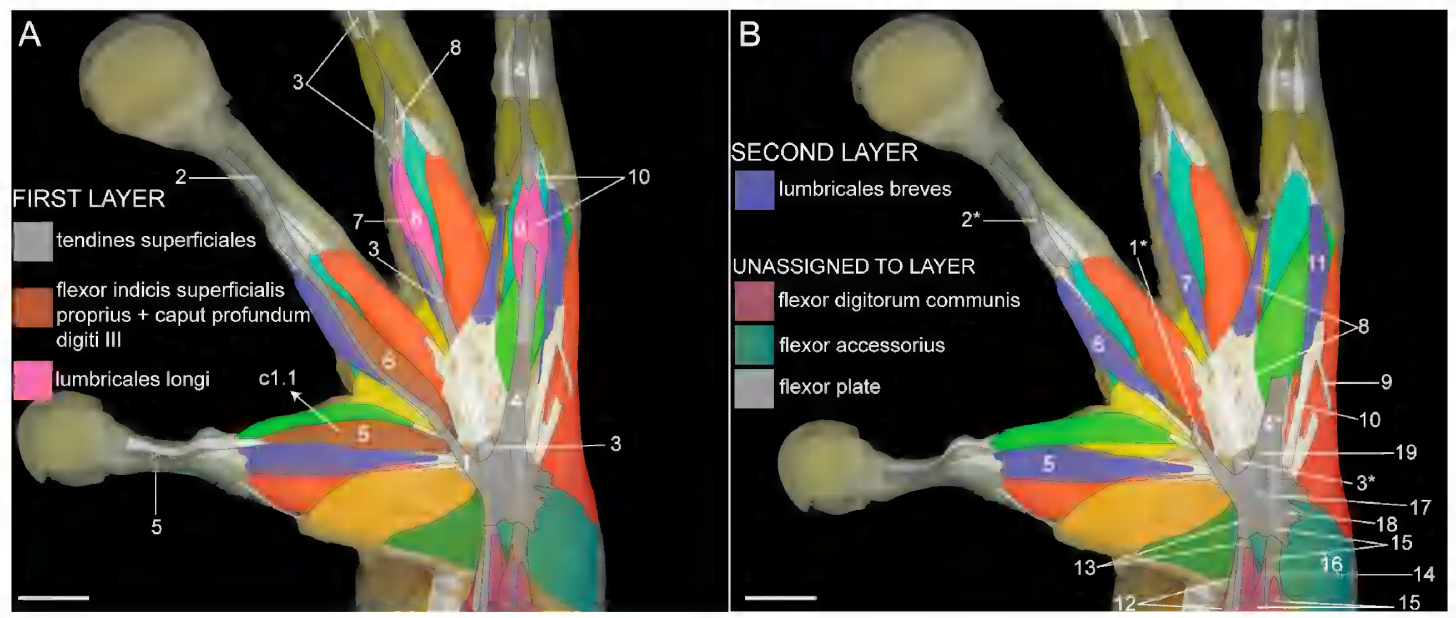

PLATE 2. Palmar surface of the left hand of Triprion petasatus (KU 296231). A. First layer: tendines superficiales, $\mathrm{m}$. flexor indicis superficialis proprius, $\mathrm{m}$. caput profundum digiti III, and mm. lumbricales longi. Elements figured: 1, tendo superficialis digiti III. 2, fused tendo superficialis digiti III with tendon of insertion of $\mathrm{m}$. caput profundum digiti III. 3, tendo superficialis digiti IV. 4, tendo superficialis digiti V. 5, m. flexor indicis superficialis proprius. 6 , m. caput profundum digiti III. 7, m. lumbricalis longus digiti IV: medial slip. 8, m. lumbricalis longus digiti IV: lateral slip. 9, m. lumbricalis longus digiti V: medial slip. 10, m. lumbricalis longus digiti V: lateral slip. B. Second layer: $\mathrm{mm}$. lumbricales breves. The forearm muscles $\mathrm{m}$. flexor digitorum communis and $\mathrm{m}$. flexor accessorius are also shown. Elements figured: $\mathbf{1}^{*}$, tendo superficialis digiti III. $\mathbf{2}^{*}$, fused tendo superficialis digiti III with tendon of insertion of $\mathrm{m}$. caput profundum digiti III. $3^{\star}$, tendo superficialis digiti IV. $4^{\star}$, tendo superficialis digiti V. $5, \mathrm{~m}$. lumbricalis brevis indicis. $6, \mathrm{~m}$. lumbricalis brevis digiti III. 7, medial m. lumbricalis brevis digiti IV. 8, medial m. lumbricalis brevis digiti V (note the flat wide and discontinuous tendon of origin). 9, lateral $\mathrm{m}$. lumbricalis brevis digiti $\mathrm{V}$ : slip from integument: tendon of origin. 10, lateral $\mathrm{m}$. lumbricalis brevis digiti $\mathrm{V}$ : slip from distal carpal 3-4-5: tendon of origin. 11, lateral m. lumbricalis brevis digiti V: fused slips from integument and distal carpal 3-4-5. 12, m. flexor digitorum communis: medial slip: portion that connects with the tendo superficialis digiti $\mathrm{V}$ (its tendon of insertion goes dorsally through the flexor plate and connects with tendo superficialis digiti $\mathrm{V}$ dorsally; not visible nor depicted with a dashed line). 13, m. flexor digitorum communis: medial slip: portion that connects with the tendo superficialis digiti IV (its tendon of insertion is partially visible by transparency through the flexor plate and depicted as a dashed line). 14, $\mathrm{m}$. flexor digitorum communis: medial slip: portion that inserts on flexor plate. 15, m. flexor digitorum communis: lateral slip (connects with tendo superficialis digiti V; its tendon of insertion is partially visible by transparency through the flexor plate and depicted as a dashed line). 16, $\mathrm{m}$. flexor accessorius. 17, m. flexor accessorius: tendon of insertion that connects with tendo superficialis digiti IV (partially visible by transparency through the flexor plate and depicted as a dashed line). 18, flexor plate. 19 , tendo superficialis digiti V: origin from flexor plate. 


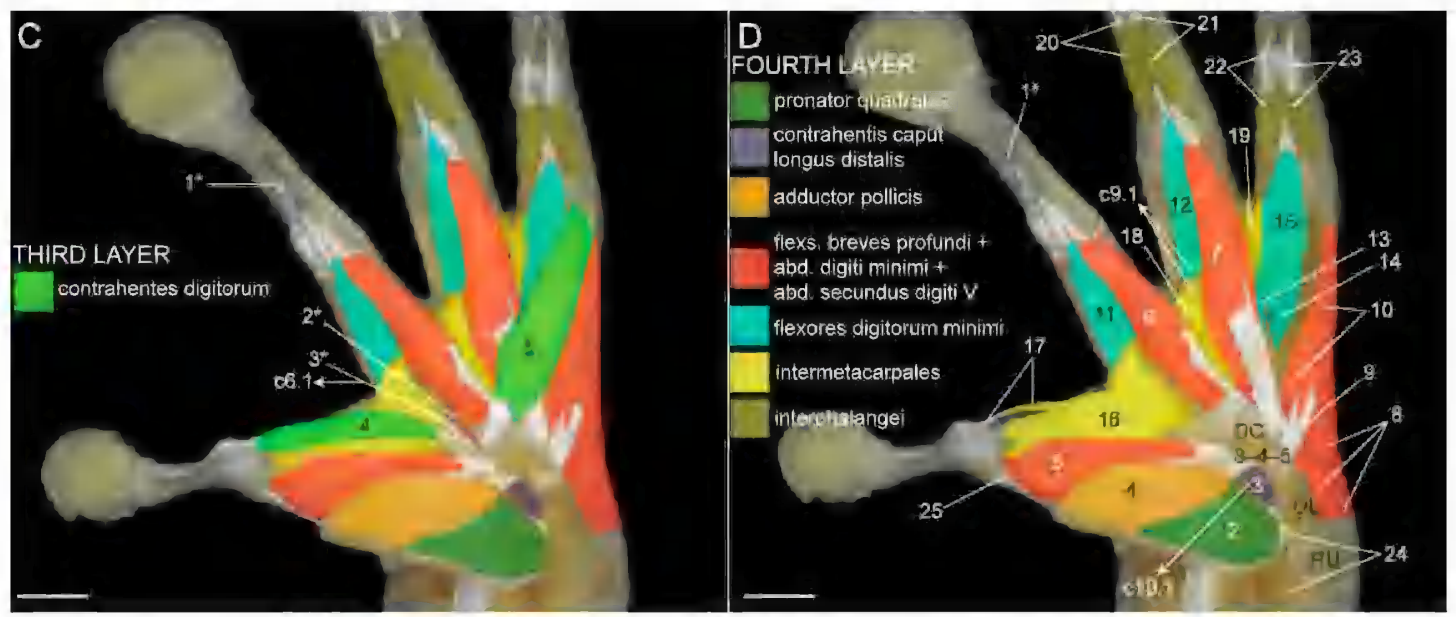

PLATE 2. (continued) C. Third layer: mm. contrahentes (only those of digits II and V are present in this species). Elements figured: $\mathbf{1}^{*}$, fused tendo superficialis digiti III with tendon of insertion of the $\mathrm{m}$. caput profundum digiti III. $2^{*}, \mathrm{~m}$. caput profundum digiti III: proximal unremoved portion. $3^{*}, \mathrm{~m}$ lumbricalis brevis digiti III: unremoved tendon of origin (from distal carpal 3-4-5). 4, m. contrahentis indicis. 5, m. contrahentis digiti V. D. Fourth layer: $\mathrm{m}$. pronator quadratus, m. contrahentis caput longus distalis, m. adductor pollicis, $\mathrm{mm}$. flexores breves profundi, $\mathrm{m}$. abductor digiti minimi, $\mathrm{m}$. abductor secundus digiti $\mathrm{V}$ (fused with the $\mathrm{m}$. abductor digiti minimi in this species), mm. flexores digitorum minimi, mm. intermetacarpales, and mm. interphalangei. Elements figured: $1^{*}$, fused tendo superficialis digiti III with tendon of insertion of the $\mathrm{m}$. caput profundum digiti III. 2, m. pronator quadratus. 3, m. contrahentis caput longus distalis. 4, m. adductor pollicis. 5, medial $\mathrm{m}$. flexor indicis brevis profundus. 6, m. flexor brevis profundus digiti III. 7, $\mathrm{m}$. flexor brevis profundus digiti IV. 8, fused $\mathrm{m}$. abductor digiti minimi and $\mathrm{m}$. abductor secundus digiti V: portion that topologically corresponds to the latter. 9 , fused $\mathrm{m}$. abductor digiti minimi and $\mathrm{m}$. abductor secundus digiti $\mathrm{V}$ : tendon of origin on distal carpal 3-4-5: portion that topologically corresponds to the former. 10, fused m. abductor digiti minimi and $\mathrm{m}$. abductor secundus digiti $\mathrm{V}$ : fleshy portion that topologically corresponds to the former. 11, m. flexor minimus digiti III. 12, m. flexor minimus digiti IV. 13, m. flexor minimus digiti V: portion/head with origin on metacarpal IV. 14, $\mathrm{m}$. flexor minimus digiti V: portion/head with origin on metacarpal V. 15, m. flexor minimus digiti V. 16, m. intermetacarpalis I: portion inserting on metacarpal II. 17, m. intermetacarpalis I: portion inserting on metacarpophalangeal joint. 18, m. intermetacarpalis II. 19, m. intermetacarpalis III. 20, m. interphalangeus digiti IV: medial slip. 21, m. interphalangeus digiti IV: lateral slip. 22, m. interphalangeus digiti $\mathrm{V}$ : medial slip. 23, m. interphalangeus digiti $\mathrm{V}$ : lateral slip. 24, $\mathrm{m}$. contrahentium caput longus proximalis: fleshy portion (delimited by a dark line) and tendon of insertion (shaded and labeled for discussion; this forearm muscle is not part of the muscles studied here). 25, ligament or strong connective tissue joining the distal end of prepollex and metacarpophalangeal joint of digit II (some medial fibers of the medial $\mathrm{m}$. flexor indicis brevis profundus inserts on it). The tendons of insertion of the $\mathrm{m}$. contrahentis indicis and $\mathrm{mm}$. flexores digitorum minimi pass through an internal channel of the metacarpophalangeal joint of their respective digit; since the connective tissue that forms these channels are thin and translucent, we shaded these tendons without discontinuities (for example, see pl. 8C, shaded indicating its apparent discontinuity as the tendons pass through the channel). Similarly, the tendons of insertion of the $\mathrm{mm}$. interphalangei pass through an internal channel of the interphalangeal joints, but we shaded these tendons without discontinuities based on the same grounds. Asterisks $\left(^{*}\right)$ indicate unremoved muscles from more superficial muscle layers other than the one being exposed. Characters figured: $\mathrm{cl} .1$, presence of $\mathrm{m}$. flexor indicis superficialis proprius (pl. 2A). c6.1, presence of the slip of the $\mathrm{m}$. lumbricalis brevis digiti III originating from the distal carpals (note the unremoved tendon of origin from distal carpal 3 4-5; pl. 2C). c9.1, $\mathrm{m}$. flexor minimus digiti IV dorsal to the $\mathrm{m}$. intermetacarpalis II (pl. 2D), c10.1, presence of m. contrahentis caput longus distalis (pl. 2D). Scale bars $=1 \mathrm{~mm}$. 


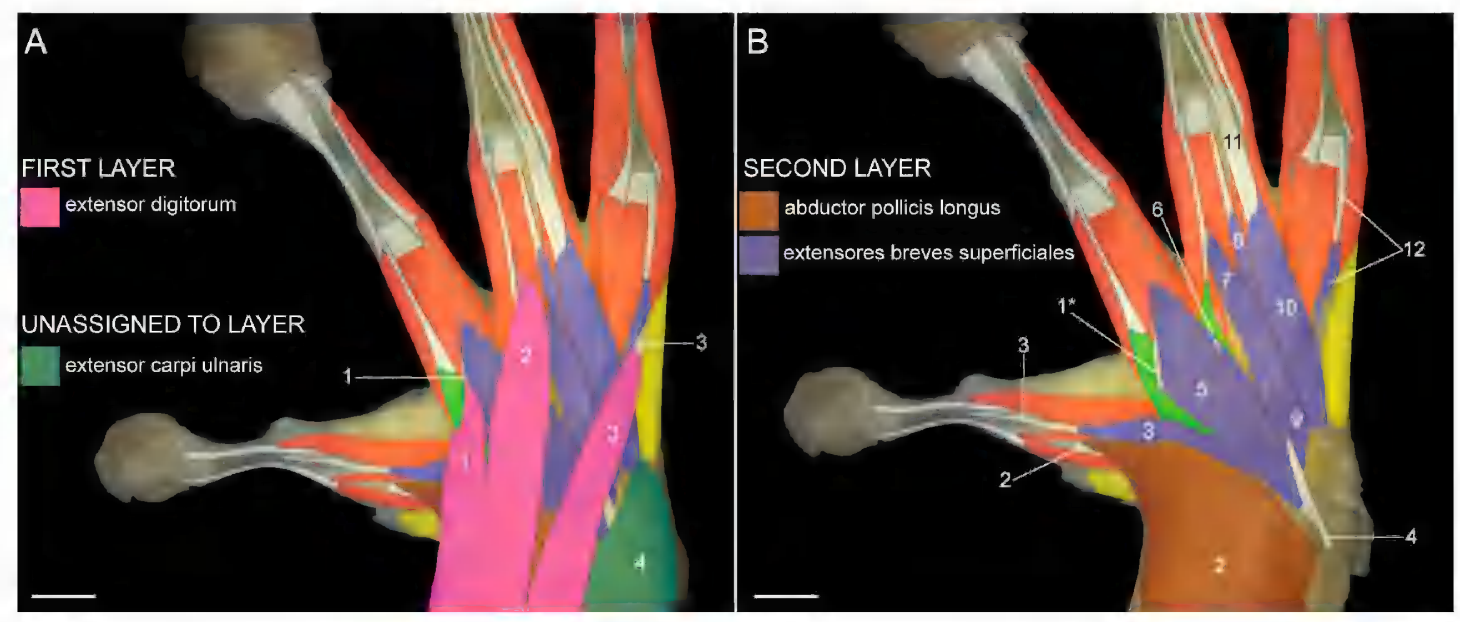

PLATE 3. Dorsal surface of the left hand (reflected for easier comparison with the remainder plates of the study) of Triprion petasatus (KU 296231). A. First layer: m. extensor digitorum; the m. extensor carpi ulnaris is also shown. Elements figured: 1-3, m. extensor digitorum: slips to digit III-V, respectively. 4, m. extensor carpi ulnaris. B. Second layer: $\mathrm{m}$. abductor pollicis longus and $\mathrm{mm}$. extensores breves superficiales. Elements figured: $\mathbf{1}^{\star}, \mathrm{m}$. extensor digitorum: slip to digit III: unremoved tendon of insertion (to metacarpal III; note the medial fibers of the $\mathrm{m}$. extensor brevis superficialis digiti III [slip from radioulna] inserting on it). 2, m. abductor pollicis longus. 3, m. extensor indicis brevis superficialis. 4, mm. extensores breves superficiales digitorum III and IV: slips from radioulna: common tendon of origin. 5, m. extensor brevis superficialis digiti III: slip from radioulna. 6, m. extensor brevis superficialis digiti III: slip from ulnare. 7, m. extensor brevis superficialis digiti IV: slip from radioulna: portion inserting on metacarpal IV. 8, m. extensor brevis superficialis digiti IV: slip from radioulna: portion inserting on metacarpophalangeal joint. 9, m. extensor brevis superficialis digiti IV: slip from ulnare. 10-11, m. extensor brevis superficialis digiti IV: slip from distal carpal 3-4-5 and tendon of insertion (which converges with the common tendon of insertion of the slips from metacarpal IV and V of the lateral $\mathrm{m}$. dorsometacarpalis proximalis digiti IV). 12, $\mathrm{m}$. extensor brevis superficialis digiti $\mathrm{V}$. 

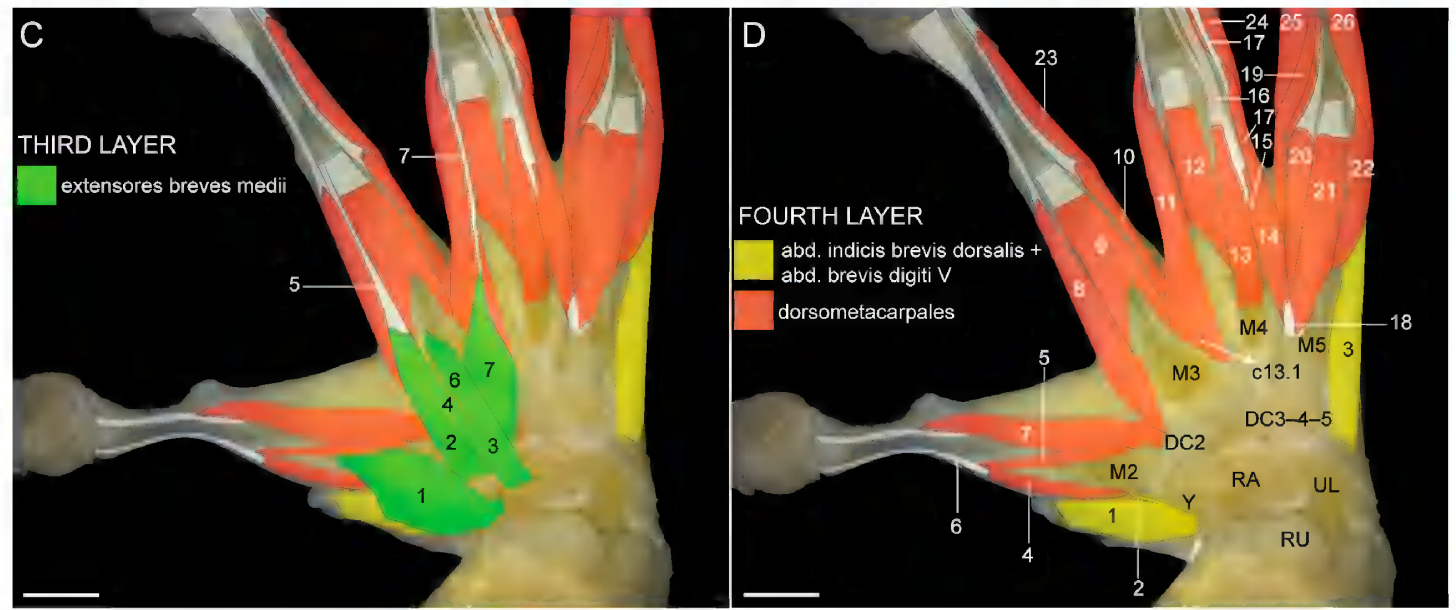

PLATE 3. (continued) C. Third layer: mm. extensores breves medii. Elements figured: 1, m. extensor indicis brevis medius. 2, m. extensor brevis medius digiti III: slip from element Y. 3, m. extensor brevis medius digiti III: slip from radiale. 4, m. extensor brevis medius digiti III: slip from radiale: portion inserting on metacarpophalangeal joint. 5, m. extensor brevis medius digiti III: slips from element $Y$ and radiale: common tendon of insertion on metacarpophalangeal joint. 6, m. extensor brevis medius digiti III: slip from radiale: portion inserting on metacarpal III. 7, m. extensor brevis medius digiti IV. D. Fourth layer: m. abductor indicis brevis dorsalis, $\mathrm{m}$. abductor brevis digiti $\mathrm{V}, \mathrm{mm}$. dorsometacarpales proximales, and $\mathrm{mm}$. dorsometacarpales distales. Elements figured: 1, m. abductor indicis brevis dorsalis: slip inserting on prepollex. 2, m. abductor indicis brevis dorsalis: slip inserting on metacarpal II. 3, m. abductor brevis digiti V. 4, medial m. dorsometacarpalis indicis proximalis: slip from medial surface of metacarpal II. 5, medial m. dorsometacarpalis indicis proximalis: slip from dorsal surface of metacarpal II. 6, medial $\mathrm{m}$. dorsometacarpalis indicis proximalis: slips from medial and dorsal surface of metacarpal II: common tendon of insertion. 7, lateral $\mathrm{m}$. dorsometacarpalis indicis proximalis. 8, medial $\mathrm{m}$. dorsometacarpalis proximalis digiti III: slips from distal carpal 2 and metacarpal III (not differentiable in the figure). 9, intermediate $\mathrm{m}$. dorsometacarpalis proximalis digiti III. 10, lateral $\mathrm{m}$. dorsometacarpalis proximalis digiti III. 11, medial $\mathrm{m}$. dorsometacarpalis proximalis digiti IV: slip from metacarpal III. 12, intermediate $\mathrm{m}$. dorsometacarpalis proximalis digiti IV. 13, lateral $\mathrm{m}$. dorsometacarpalis proximalis digiti IV: slip from metacarpal IV. 14, lateral $\mathrm{m}$. dorsometacarpalis proximalis digiti IV: slip from metacarpal V. 15, lateral $\mathrm{m}$. dorsometacarpalis proximalis digiti IV: slip from metacarpal V: portion inserting on the proximal interphalangeal joint by a common tendon with the slip from metacarpal IV of the lateral $\mathrm{m}$. dorsometacarpalis proximalis digiti IV. 16, lateral $\mathrm{m}$. dorsometacarpalis proximalis digiti IV: slips from metacarpal IV and V: common tendon of insertion on proximal interphalangeal joint. 17, lateral $\mathrm{m}$. dorsometacarpalis proximalis digiti IV: slip from metacarpal V: portion inserting on the distal interphalangeal joint/distal phalanx. 18, common tendon of origin of (i) slip from metacarpal $\mathrm{V}$ of the lateral $\mathrm{m}$. dorsometacarpalis proximalis digiti IV, (ii) part of the slip from metacarpal $\mathrm{V}$ of the medial $\mathrm{m}$. dorsometacarpalis proximalis digiti $\mathrm{V}$, and (iii) part of the intermediate $\mathrm{m}$. dorsometacarpalis proximalis digiti V. 19, medial $\mathrm{m}$. dorsometacarpalis proximalis digiti V: slip from metacarpal IV. 20, medial $\mathrm{m}$. dorsometacarpalis proximalis digiti V: slip from metacarpal V. 21, intermediate $\mathrm{m}$. dorsometacarpalis proximalis digiti V. 22, lateral $\mathrm{m}$. dorsometacarpalis proximalis digiti V. $\mathbf{2 3}$, lateral $\mathrm{m}$. dorsometacarpalis distalis digiti III. $\mathbf{2 4}$, lateral $\mathrm{m}$. dorsometacarpalis distalis digiti IV. 25, medial m. dorsometacarpalis distalis digiti V. 26, lateral m. dorsometacarpalis distalis digiti V. Asterisks $\left({ }^{*}\right)$ indicate unremoved muscles from more superficial muscle layers other than the one being exposed. Characters figured: c13.1, presence of the slip from metacarpal III of the medial $\mathrm{m}$. dorsometacarpalis proximalis digiti IV (pl. 3D). Scale bars $=1 \mathrm{~mm}$. 

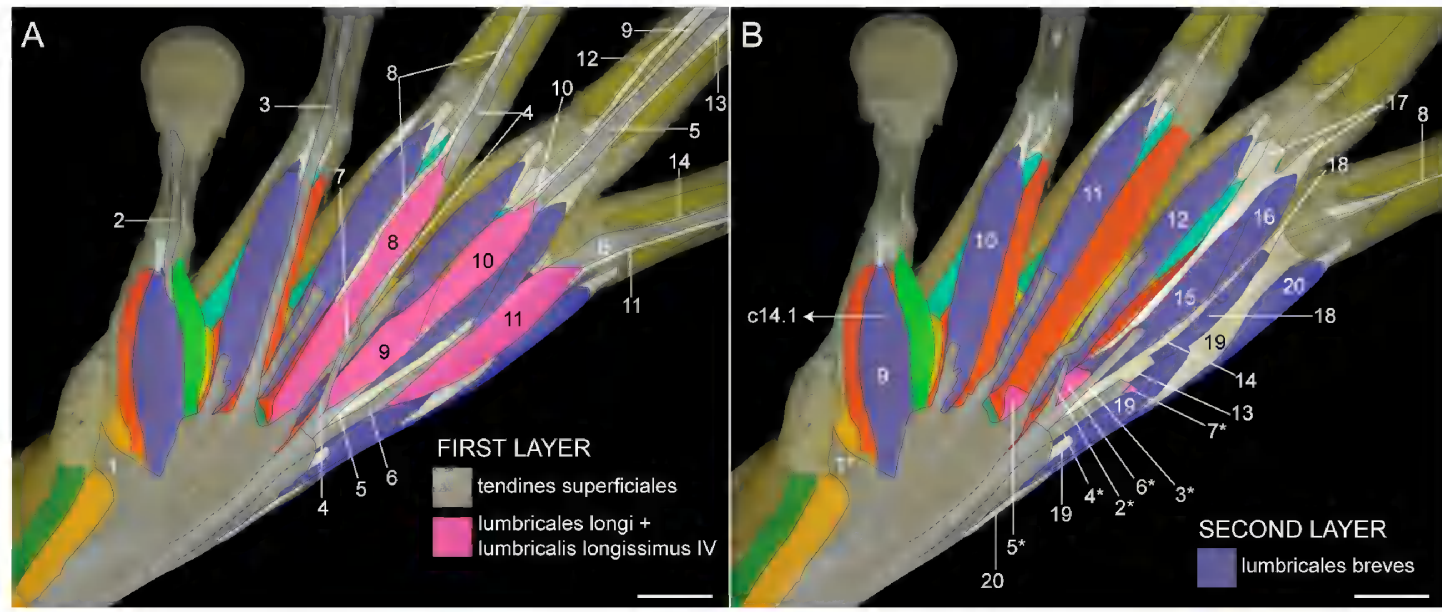

PLATE 4. Plantar surface of the left foot of Triprion petasatus (KU 296231). A. First layer: tendines superficiales, $\mathrm{mm}$. lumbricales longi, and $\mathrm{m}$. lumbricalis longissimus digiti IV. Elements figured: 1-6, tendo superficialis praehallucis, tendo superficialis hallucis (proximally hidden by the $\mathrm{m}$. lumbricalis brevis hallucis) and tendines superficiales digitorum II-V, respectively. 7, m. lumbricalis longus digiti III: supplementary tendon of origin from the tendo superficialis digiti III. 8, m. lumbricalis longus digiti III. 9, m. lumbricalis longus digiti IV. 10, $\mathrm{m}$. lumbricalis longissimus digiti IV. 11, m. lumbricalis longus digiti V. 12, ligament joining the medioplantar portion of the metatarsophalangeal joint and the medioplantar portion of the proximal interphalangeal joint of digit IV. 13, ligament joining the lateroplantar portion of the metatarsophalangeal joint and the lateroplantar portion of the proximal interphalangeal joint of digit IV. 14, ligament joining the medioplantar portion of the metatarsophalangeal joint and medioplantar portion of the proximal interphalangeal joint of digit V. B. Second layer: $\mathrm{mm}$. lumbricales breves. Elements figured: $\mathbf{1}^{\star}$, tendo superficialis praehallucis. $\mathbf{2}^{\star}-\mathbf{4}^{\star}$, tendines superficiales digitorum III-V: proximal unremoved portion, respectively. $5^{\star}-7^{\star}, \mathrm{mm}$. lumbricales longi digitorum III-V: proximal unremoved portion, respectively. 8, ligament joining the medioplantar portion of the metatarsophalangeal joint and medioplantar portion of the proximal interphalangeal joint of digit $\mathrm{V} . \mathbf{9}, \mathrm{m}$. lumbricalis brevis hallucis. 10, m. lumbricalis brevis digiti II. 11, m. lumbricalis brevis digiti III. 12, medial $\mathrm{m}$. lumbricalis brevis digiti IV. 13, lateral $\mathrm{m}$. lumbricalis brevis digiti IV and medial $\mathrm{m}$. lumbricalis brevis digiti V (medial slip): common tendon of origin. 14, lateral $\mathrm{m}$. lumbricalis brevis digiti IV: tendon of origin. 15, lateral $\mathrm{m}$. lumbricalis brevis digiti IV: main portion (from plantar cartilage). 16, lateral $\mathrm{m}$. lumbricalis brevis digiti IV: distal and superficial portion with origin from integument. 17, lateral $\mathrm{m}$. lumbricalis brevis digiti IV: tendon of insertion on metatarsophalangeal joint (the dashed line that seems to be a continuation of it corresponds to the $\mathrm{m}$. flexor minimus digiti IV, see element number 16 of the fourth layer). 18, medial $\mathrm{m}$. lumbricalis brevis digiti V: medial slip. 19, medial m. lumbricalis brevis digiti V: lateral slip. 20, lateral m. lumbricalis brevis digiti V. C. Third layer: $\mathrm{mm}$. contrahentes pedis (only the muscle of digit I is present in this species); the aponeurosis plantaris, $\mathrm{mm}$. flexores breves superficiales (only its tendon of insertion is partially visible by transparency of the aponeurosis plantaris, which is depicted as a dashed line), and $\mathrm{m}$. flexor accessorius distalis (only a distal portion visible) are also shown. Elements figured: $\mathbf{1}^{\star}$, tendo superficialis praehallucis. $2^{\star}-4^{\star}$, tendines superficiales digitorum III-V: proximal unremoved portion, respectively. $5^{\star}-6^{\star}, \mathrm{mm}$. lumbricales longi digitorum IV-V: proximal unremoved portion, respectively. $7^{*}$, lateral m. lumbricalis brevis digiti IV and medial $\mathrm{m}$. lumbricalis brevis digiti $\mathrm{V}$ (medial slip): common tendon of origin. $\mathbf{8}^{\star}$, lateral $\mathrm{m}$. lumbricalis brevis digiti IV: proximal unremoved portion. $9^{*}$, medial $\mathrm{m}$. lumbricalis brevis digiti $\mathrm{V}$ : lateral slip: proximal unremoved portion. 10, ligament joining the medioplantar portion of the metatarsophalangeal joint and the medioplantar portion of the proximal interphalangeal joint of digit V. 11, m. contrahentis pedis hallucis. 12, m. contrahentis pedis hallucis: tendon of insertion (the dashed line indicates the portion that pass through an internal channel of the metatarsophalangeal joint). 

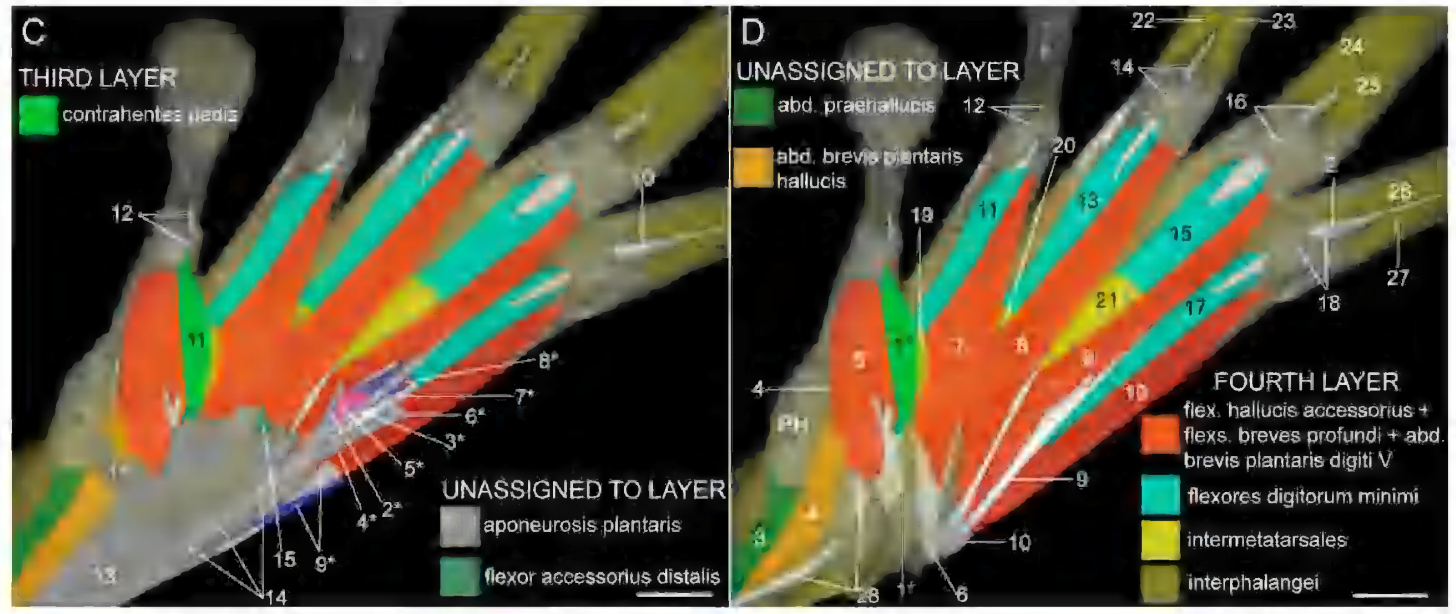

PLATE 4. (continued) 13, aponeurosis plantaris. 14, mm. flexores breves superficialis: tendon of insertion (partially visible by transparency through the aponeurosis plantaris; depicted as a dashed line), proximally to its split into the tendines superficiales digitorum III-V. 15, m. flexor accessorius distalis: distal portion inserting proximally on tendo superficialis digiti II (the remaining portion of the flexor accessorius distalis and the flexor accessorius proximalis are hidden by the aponeurosis plantaris). D. Fourth layer: $\mathrm{m}$. flexor hallucis accessorius, $\mathrm{mm}$. flexores breves profundi, $\mathrm{m}$. abductor brevis plantaris digiti $\mathrm{V}, \mathrm{mm}$. flexores digitorum minimi, $\mathrm{mm}$. intermetatarsales, and $\mathrm{mm}$. interphalangei. The $\mathrm{m}$. abductor praehallucis and $\mathrm{m}$. abductor brevis plantaris hallucis are also shown. Elements figured: $\mathbf{1}^{*}, \mathrm{~m}$. contrahentis pedis hallucis. 2, ligament joining the medioplantar portion of the metatarsophalangeal joint and the medioplantar portion of the proximal interphalangeal joint of digit V. 3, m. abductor praehallucis (its origin is from the aponeurosis plantaris, removed in the previous step, and not from the tendon of insertion of the $\mathrm{m}$. interosseus cruris, as it appears to be in the figure). $4, \mathrm{~m}$. abductor brevis plantaris hallucis (its origin is from the aponeurosis plantaris, same comments as for the $\mathrm{m}$. abductor praehallucis). $5, \mathrm{~m}$. flexor hallucis accessorius. $6, \mathrm{~mm}$. flexores breves profundi digitorum II-III: common tendon of origin. 7-9, mm. flexores breves profundi digitorum II-IV. 10, $\mathrm{m}$. abductor brevis plantaris digiti V. 11, m. flexor minimus digiti II. 12, m. flexor minimus digiti II: tendon of insertion (the dashed line indicates the portion that pass through an internal channel of the metatarsophalangeal joint). 13. m. flexor minimus digiti III. 14, $\mathrm{m}$. flexor minimus digiti III: tendon of insertion (the dashed line indicates the portion that pass through an internal channel of the metatarsophalangeal joint). 15, $\mathrm{m}$. flexor minimus digiti IV. 16, $\mathrm{m}$. flexor minimus digiti IV: tendon of insertion (the dashed line indicates the portion that pass through an internal channel of the metatarsophalangeal joint). 17, m. flexor minimus digiti V. 18, m. flexor minimus digiti $V$ : tendon of insertion (the dashed line indicates the portion that pass through an internal channel of the metatarsophalangeal joint). 19-21, mm. intermetatarsales I-III. 22-23, $\mathrm{m}$. interphalangeus digiti III: medial and lateral slips, respectively. $24-25, \mathrm{~m}$. interphalangeus proximalis digiti IV: medial and lateral slips, respectively. 26-27, $\mathrm{m}$. interphalangeus proximalis digiti $\mathrm{V}$ : medial and lateral slips, respectively. 28, $\mathrm{m}$. interosseus cruris: tendon of insertion (the discontinuity is due to the fact that it passes through a groove in the condyle of the tibiale that hides it, and reappears distally to insert on element $\mathrm{Y}$ ). Asterisks ${\left({ }^{*}\right)}^{*}$ indicate unremoved muscles from more superficial muscle layers other than the one being exposed. The morphology and proportions of the following elements are approximated, since they were not completely distinguishable in the picture for allowing an accurate shading (drawings and descriptions were employed as complement): tendo superficialis praehallucis and distal extension of the m. intermetatarsalis III. Characters figured: c14.1, presence of $\mathrm{m}$ lumbricalis brevis hallucis (pl. 4B). Scale bars $=1 \mathrm{~mm}$. 

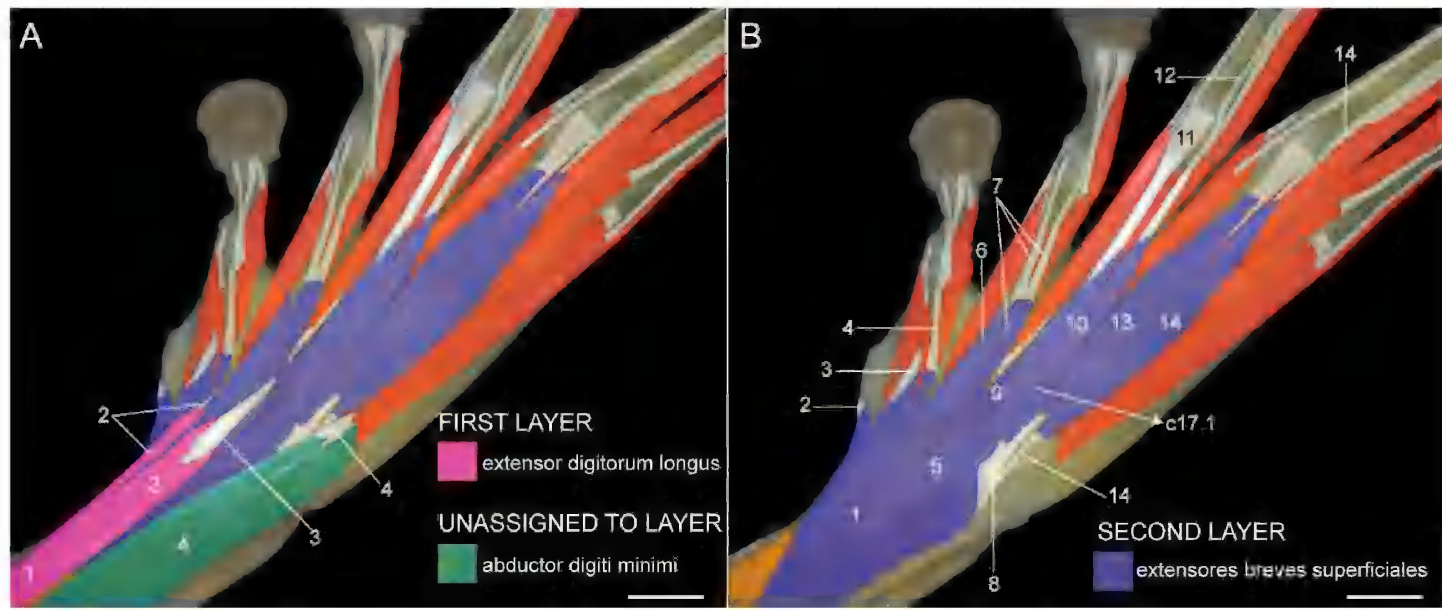

PLATE 5. Dorsal surface of the left foot (reflected for comparison with other plates) of Triprion petasatus (KU 296231). A. First layer: $\mathrm{m}$. extensor digitorum longus; the $\mathrm{m}$. abductor digiti minimi is also shown. Elements figured: 1, m. extensor digitorum longus. 2, m. extensor digitorum longus: slip to metatarsal II. 3, m. extensor digitorum longus: slip to metatarsal III. 4, m. abductor digiti minimi. B. Second layer: $\mathrm{mm}$. extensores breves superficiales. Elements figured: 1, m. extensor brevis superficialis hallucis. 2, m. extensor brevis superficialis hallucis: tendon of insertion on prehallux. 3, m. extensor brevis superficialis hallucis: tendon of insertion on metatarsal I. 4, m. extensor brevis superficialis hallucis: common tendon of insertion (on metatarsophalangeal joint) with the $\mathrm{m}$. extensor brevis medius hallucis. 5, m. extensor brevis superficialis digiti II. 6, m. extensor brevis superficialis digiti II: portion inserting on metatarsal II. 7, m. extensor brevis superficialis digiti II: portion inserting on metatarsophalangeal joint by common tendon with the $\mathrm{m}$. extensor brevis medius digiti II. 8, m. extensor brevis superficialis digiti III and medial $\mathrm{m}$. extensor brevis superficialis digiti IV: common tendon of origin. 9, m. extensor brevis superficialis digiti III; portion inserting on metatarsal III. 10, m. extensor brevis superficialis digiti III: portion inserting on metatarsophalangeal joint and proximal interphalangeal joint of digit III. 11, m. extensor brevis superficialis digiti III: tendon of insertion on metatarsophalangeal joint. 12, m. extensor brevis superficialis digiti III: tendon of insertion on proximal interphalangeal joint. 13, medial $\mathrm{m}$. extensor brevis superficialis digiti IV. 14, lateral $\mathrm{m}$. extensor brevis superficialis digiti IV. 


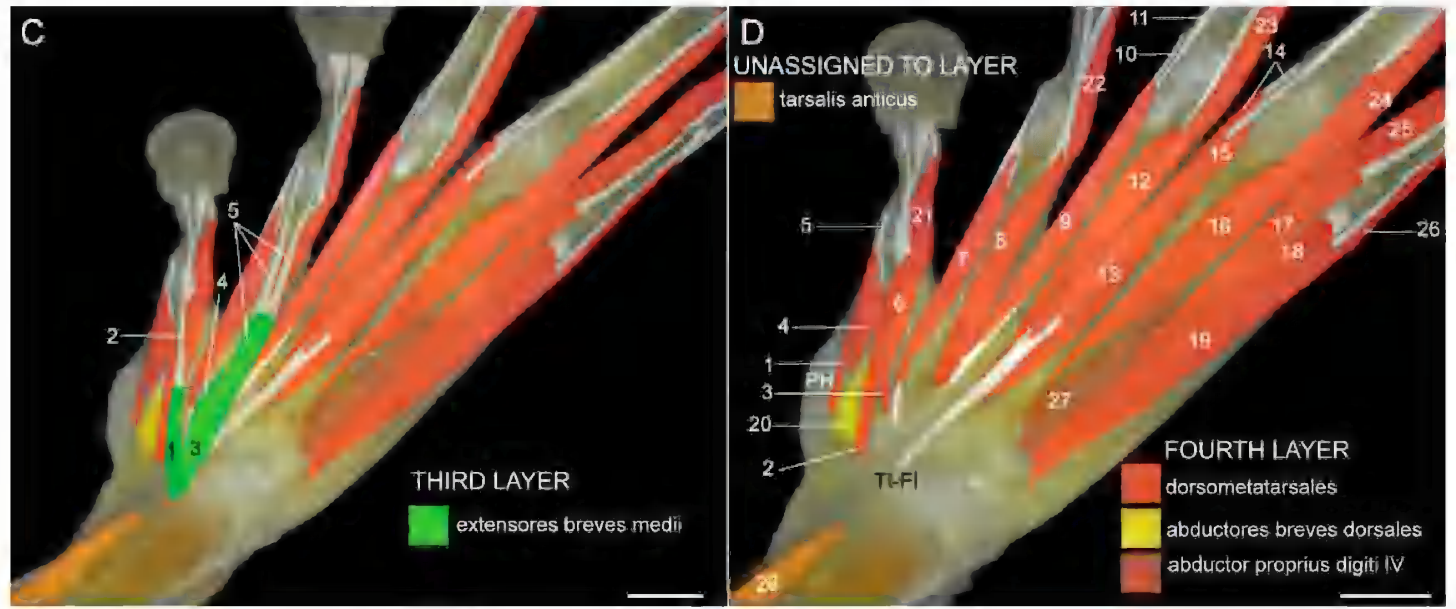

PLATE 5. (continued) C. Third layer: mm. extensores breves medii. Elements figured: $\mathbf{1}, \mathrm{m}$. extensor brevis medius hallucis. 2, m. extensor brevis medius hallucis: common tendon of insertion (on metatarsophalangeal joint) with the m. extensor brevis superficialis hallucis. 3, m. extensor brevis medius digiti II. 4, m. extensor brevis medius digiti II: portion inserting on metatarsal II. 5, m. extensor brevis medius digiti II: portion inserting on metatarsophalangeal joint by common tendon with the $\mathrm{m}$. extensor brevis superficialis digiti II. D. Fourth layer: $\mathrm{mm}$. dorsometatarsales proximales, $\mathrm{mm}$. dorsometatarsales distales, $\mathrm{mm}$. abductores breves dorsales (only the muscle of digit I is present), and $\mathrm{m}$. abductor proprius digiti IV; the $\mathrm{m}$. tarsalis anticus is also shown. Elements figured: 1, medial m. dorsometatarsalis hallucis proximalis: slip from the prehallux. 2, medial $\mathrm{m}$. dorsometatarsalis hallucis proximalis: slip from element $\mathrm{Y} .3$, medial $\mathrm{m}$. dorsometatarsalis hallucis proximalis: slip from the proximal portion of metatarsal I. 4, medial $\mathrm{m}$. dorsometatarsalis hallucis proximalis: slip from the mediodistal portion of metatarsal I. 5, medial m. dorsometatarsalis hallucis proximalis: common tendon of insertion of the four slips. 6, lateral $\mathrm{m}$. dorsometatarsalis hallucis proximalis. 7 , medial $\mathrm{m}$. dorsometatarsalis proximalis digiti II. 8, lateral $\mathrm{m}$. dorsometatarsalis proximalis digiti II. 9, medial $\mathrm{m}$. dorsometatarsalis proximalis digiti III. 10, medial $\mathrm{m}$. dorsometatarsalis proximalis digiti III: tendon of insertion on metatarsophalangeal joint. 11, medial $\mathrm{m}$. dorsometatarsalis proximalis digiti III: tendon of insertion on proximal interphalangeal joint. 12, lateral $\mathrm{m}$. dorsometatarsalis proximalis digiti III. 13, medial $\mathrm{m}$. dorsometatarsalis proximalis digiti IV. 14, medial $\mathrm{m}$. dorsometatarsalis proximalis digiti IV: portion inserting on metatarsophalangeal joint. 15, medial $\mathrm{m}$. dorsometatarsalis proximalis digiti IV: portion inserting on distal interphalangeal joint/distal phalanx. 16, lateral $\mathrm{m}$. dorsometatarsalis proximalis digiti IV. 17, medial $\mathrm{m}$. dorsometatarsalis proximalis digiti $\mathrm{V}$. 18, intermediate $\mathrm{m}$. dorsometatarsalis proximalis digiti V. 19, lateral $\mathrm{m}$. dorsometatarsalis proximalis digiti V. 20, $\mathrm{m}$. abductor brevis dorsalis hallucis. 21-24, 26, lateral $\mathrm{m}$. dorsometatarsalis hallucis distalis and lateral $\mathrm{mm}$. dorsometatarsales distales digitorum II-V, respectively. 25, medial $\mathrm{m}$. dorsometatarsalis distalis digiti V. $27, \mathrm{~m}$. abductor proprius digiti IV. $28, \mathrm{~m}$. tarsalis anticus. The morphology and proportions of the following elements are approximated, since they were not completely distinguishable in the picture for allowing an accurate shading (drawings and descriptions were employed as complement): common tendon of insertion of the $\mathrm{mm}$. extensores breves superficialis and medius digiti III; slips of the medial $\mathrm{m}$. dorsometatarsalis hallucis proximalis; tendon of insertion of the lateral $\mathrm{m}$ dorsometatarsalis hallucis proximalis, and tendon of insertion of the lateral $\mathrm{m}$. dorsometatarsalis proximalis digiti III. Characters figured: c17.1, presence of $\mathrm{m}$ extensor brevis superficialis digiti III ( $\mathrm{pl} .5 \mathrm{~B}$ ). Scale bars $=1 \mathrm{~mm}$. 


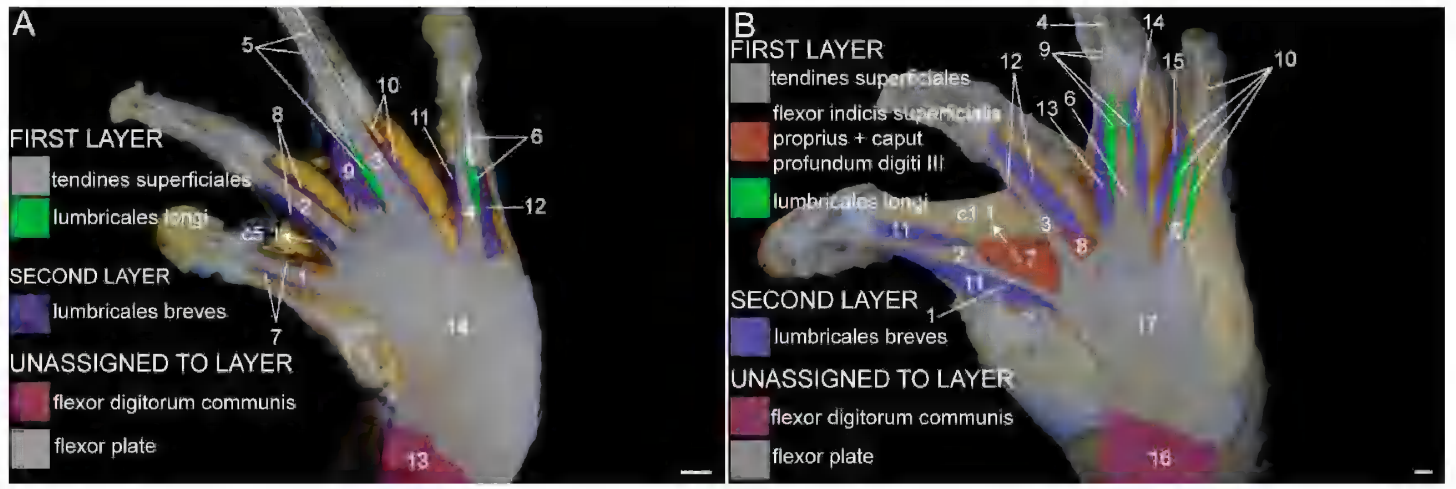

PLATE 6. Palmar surface showing the first layer of muscles. Muscles of second palmar layer (mm. lumbricales breves) and the forearm $\mathrm{m}$. flexor accessorius and $\mathrm{m}$. flexor digitorum communis are also colored for discussion. A. Right hand (reflected for comparison with other plates) of Rhinophrynus dorsalis (KU 171202). Elements figured: 1, tendo superficialis indicis. 2-4, tendines superficiales digitorum III-V. 5-6, mm. lumbricales longi digitorum IV-V. 7, m. lumbricalis brevis indicis. 8, m. lumbricalis brevis digiti III. 9, medial m. lumbricalis brevis digiti IV. 10, lateral $\mathrm{m}$. lumbricalis brevis digiti IV. 11, medial $\mathrm{m}$. lumbricalis brevis digiti V. 12, lateral $\mathrm{m}$. lumbricalis brevis digiti V. 13, m. flexor digitorum communis. 14, flexor plate (a connective tissue that covers the lateropalmar region is fused to the flexor plate and it is also gray shaded; the $\mathrm{m}$. flexor accessorius is hidden by this connective tissue). B. Left hand of Spea intermontana (KU 297558). Elements figured: 1, tendo superficialis indicis. 2, fused tendo superficialis indicis and tendon of insertion of the $\mathrm{m}$. flexor indicis superficialis proprius. 3, fused tendo superficialis digiti III and tendon of insertion of the $\mathrm{m}$. caput profundum digiti III. 4-5, tendines superficiales digitorum IV-V. 6, gap that divides the tendo superficialis digiti IV into a medial and lateral portion. $7, \mathrm{~m}$. flexor indicis superficialis proprius. 8, $\mathrm{m}$. caput profundum digiti III, 9-10, mm. lumbricales longi digitorum IV-V. 11, m. lumbricalis brevis indicis. 12, $\mathrm{m}$. lumbricalis brevis digiti III. 13, medial $\mathrm{m}$. lumbricalis brevis digiti IV. 14, lateral m. lumbricalis brevis digiti IV. 15, medial m. lumbricalis brevis digiti V. 16, m. flexor digitorum communis. 17, flexor plate (a connective tissue that covers the lateropalmar region is fused to the flexor plate and it is also gray shaded; the $\mathrm{m}$. flexor accessorius is hidden by this connective tissue). 

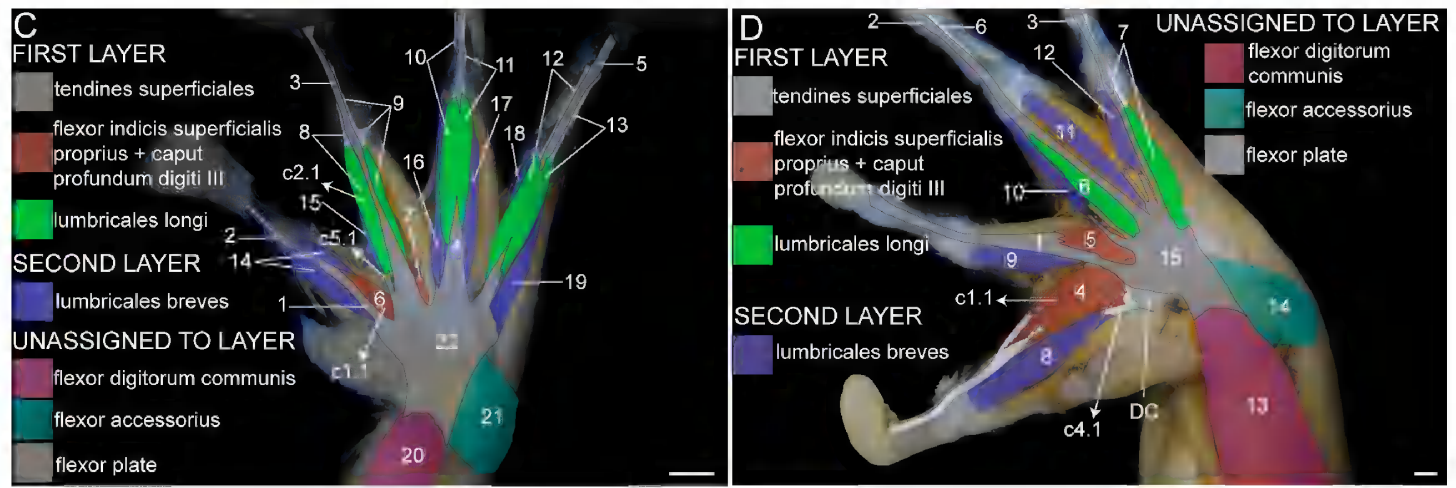

PLATE 6. (continued) C. Left hand of Heleophryne purcelli (FMNH 250016). Elements figured: 1, tendo superficialis indicis. 2, fused tendo superficialis indicis and tendon of insertion of the $\mathrm{m}$. flexor indicis superficialis proprius. 3, fused tendo superficialis digiti III and tendon of insertion of the $\mathrm{m}$. caput profundum digiti III. 4-5, tendines superficiales digitorum IV-V. 6, m. flexor indicis superficialis proprius. 7, m. caput profundum digiti III. 8, m. lumbricalis longus digiti III: medial slip. 9, m. lumbricalis longus digiti III: lateral slip (note the additional insertion on the metacarpophalangeal joint). 10, m. lumbricalis longus digiti IV: medial slip. 11, m. lumbricalis longus digiti IV: lateral slip. 12, m. lumbricalis longus digiti V: medial slip. 13, m. lumbricalis longus digiti V: lateral slip. 14, $\mathrm{m}$. lumbricalis brevis indicis. 15, m. lumbricalis brevis digiti III. 16, medial $\mathrm{m}$. lumbricalis brevis digiti IV. 17, lateral $\mathrm{m}$. lumbricalis brevis digiti IV. 18, medial m. lumbricalis brevis digiti V. 19, lateral $\mathrm{m}$. lumbricalis brevis digiti V. 20, $\mathrm{m}$. flexor digitorum communis. 21, m. flexor accessorius. 22, flexor plate. D. Left hand of Tomopterna delalandii (KU 207900). Elements figured: 1, fused tendo superficialis digiti III and tendon of insertion of the $\mathrm{m}$. caput profundum digiti III. 2-3, tendines superficiales digitorum IV-V. 4, m. flexor indicis superficialis proprius. 5, m. caput profundum digiti III. 6-7, $\mathrm{mm}$. lumbricales longi digitorum IV-V. 8, m. lumbricalis brevis indicis. 9, m. lumbricalis brevis digiti III. 10, medial $\mathrm{m}$. lumbricalis brevis digiti IV. 11, lateral $\mathrm{m}$. lumbricalis brevis digiti IV. 12, medial $\mathrm{m}$. lumbricalis brevis digiti V. 13, m. flexor digitorum communis. 14, m. flexor accessorius. 15, flexor plate. Characters figured: $\mathrm{c} 1.1$, presence of $\mathrm{m}$. flexor indicis superficialis proprius ( $\mathrm{pl} .6 \mathrm{~B}-\mathrm{D}$ ). $\mathrm{c} 2.1$, presence of $\mathrm{m}$. lumbricalis longus digiti III (pl. 6C). c4.1, presence of the origin from distal carpals of the m. lumbricalis brevis indicis (pl. 6D). c5.1, presence of the slip of the $\mathrm{m}$. lumbricalis brevis digiti III originating from flexor plate and/or tendo superficialis digiti III (pl. 6A, C). Scale bars $=1 \mathrm{~mm}$. 


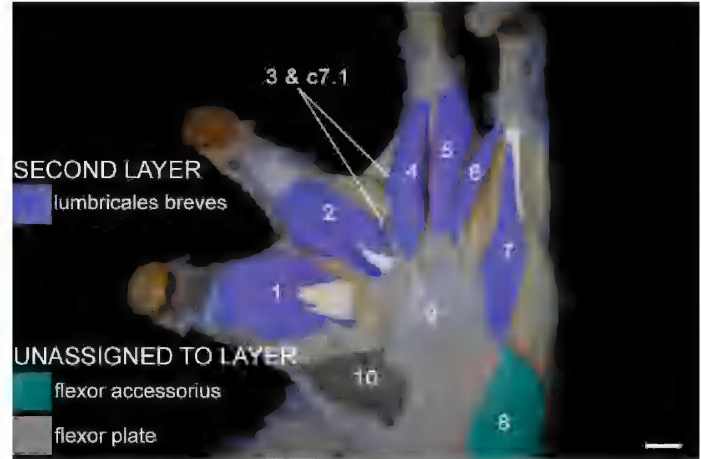

PLATE 7. Palmar surface showing the second layer of muscles ( $\mathrm{mm}$. lumbricales breves) of the left hand of Scutiger mammatus (FMNH 282284). The proximal portion of the tendon of origin of the supplementary slip of the medial $\mathrm{m}$. lumbricalis brevis digiti IV is not shaded because it is very thin and fused to the fascia of the $\mathrm{m}$. lumbricalis brevis digiti III. The flexor accessorius and the flexor plate are also shown. Elements figured: 1, $\mathrm{m}$. lumbricalis brevis indicis. 2, m. lumbricalis brevis digiti III. 3, medial $\mathrm{m}$. lumbricalis brevis digiti IV: supplementary medial slip form distal carpals. 4, medial m. lumbricalis brevis digiti IV. 5, lateral $\mathrm{m}$. lumbricalis brevis digiti IV. 6, medial $\mathrm{m}$. lumbricalis brevis digiti $\mathrm{V}$. 7, lateral $\mathrm{m}$. lumbricalis brevis digiti $\mathrm{V}$ (tentative identification and color; most palmar muscles of digit $\mathrm{V}$ are totally or partially fused, obscuring their identities). 8, m. flexor accessorius. 9, flexor plate. 10 , unidentified muscle with origin from the flexor plate and insertion on the prepollex. Characters figured: $c 7.1$, supplementary slip of the medial $\mathrm{m}$. lumbricalis brevis digiti IV from distal carpals.
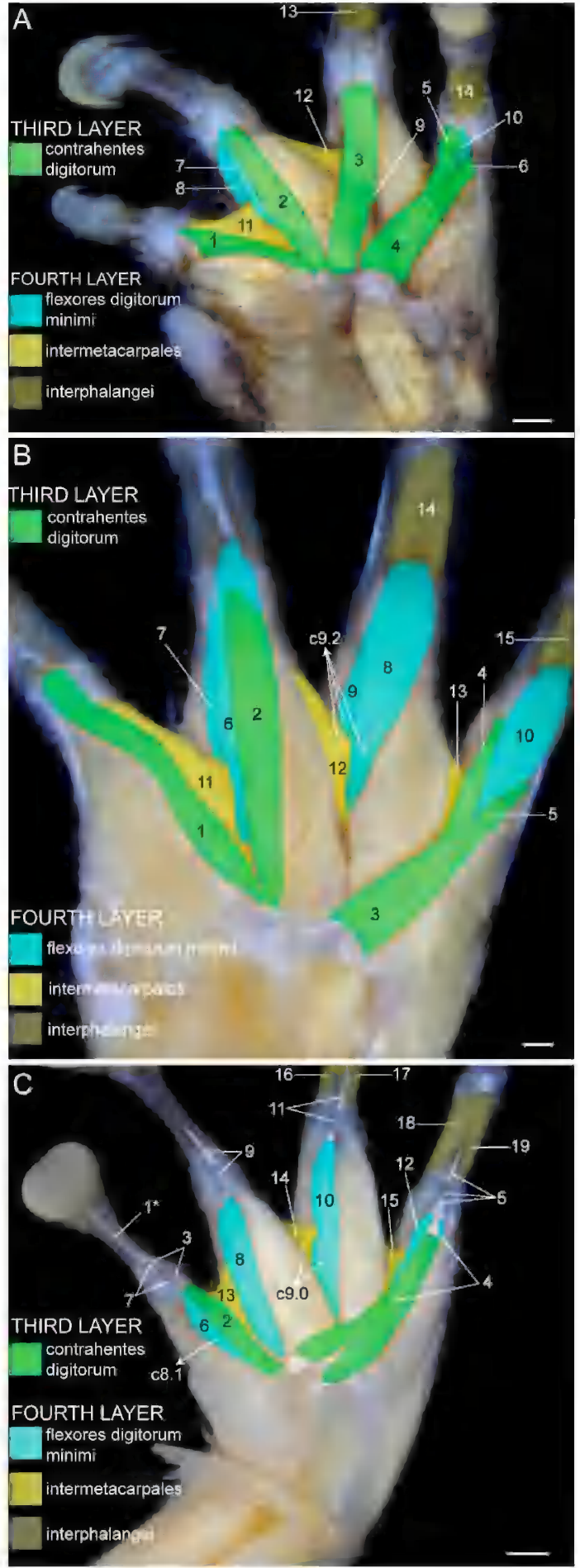
PLATE 8. Palmar surface showing the third layer of muscles: $\mathrm{mm}$. contrahentes digitorum. Some muscles of the fourth layer ( $\mathrm{mm}$. flexores digitorum minimi, intermetacarpales, and interphalangei) are also colored for discussion. A. Left hand of Bombina orientalis (KU 38654). Elements figured: 1, m. contrahentis indicis. 2-4, $\mathrm{mm}$. contrahentes digitorum III-V. 5, m. contrahentis digiti V: medial insertion on metacarpal V (medially to the $\mathrm{m}$. flexor minimus digiti V). 6, m. contrahentis digiti V: lateral insertion on metacarpal $\mathrm{V}$ (laterally to the $\mathrm{m}$. flexor minimus digiti V). $7, \mathrm{~m}$. flexor minimus digiti III: ventral origin/head with respect to the $\mathrm{m}$. intermetacarpalis I. 8, m. flexor minimus digiti III: dorsal origin/head with respect to the $\mathrm{m}$. intermetacarpalis I. 9, m. flexor minimus digiti IV: ventral origin/head with respect to the $\mathrm{m}$. intermetacarpalis II (almost completely hidden by the $\mathrm{m}$. contrahentis digiti IV; compare with $\mathrm{pl} .9 \mathrm{~B}) . \mathbf{1 0}, \mathrm{m}$. flexor minimus digiti V. 11-12, mm. intermetacarpales I-II. 13-14, $\mathrm{mm}$. interphalangei digitorum IV-V: medial and lateral slips fused into a single muscular body. B. Right hand (reflected for comparison with other plates) of Barbourula busuangensis (KU 324606). Elements figured: 1, m. contrahentis indicis. 2-3, mm. contrahentes digitorum III and $\mathrm{V} .4, \mathrm{~m}$. contrahentis digiti $\mathrm{V}$ : medial insertion on metacarpal $\mathrm{V}$ (medially to the $\mathrm{m}$. flexor minimus digiti $\mathrm{V}$ ). $5, \mathrm{~m}$. contrahentis digiti $\mathrm{V}$ : lateral insertion on metacarpal $\mathrm{V}$ (laterally to the $\mathrm{m}$. flexor minimus digiti $\mathrm{V}$ ). 6 , $\mathrm{m}$. flexor minimus digiti III: ventral origin/head with respect to the $\mathrm{m}$. intermetacarpalis I, 7, m. flexor minimus digiti III: dorsal origin/head with respect to the m. intermetacarpalis I. 8, m. flexor minimus digiti IV: ventral origin/head with respect to the $\mathrm{m}$. intermetacarpalis II. 9, m. flexor minimus digiti IV: dorsal origin/ head with respect to the m. intermetacarpalis II. 10, m. flexor minimus digiti V. 11-13, mm. intermetacarpales I-III. 14-15, mm. interphalangei digitorum IV-V: medial and lateral slips fused into a single muscular body. C. Right hand (reflected for comparison with other plates) of Heleophryne orientalis (FMNH 187311). Elements figured: $\mathbf{1}^{*}$, fused tendo superficialis indicis and tendon of insertion of the $\mathrm{m}$. flexor indicis superficialis proprius: distal unremoved portion. $2, \mathrm{~m}$. contrahentis indicis. $3, \mathrm{~m}$. contrahentis indicis: tendon of insertion (the dashed line indicates the portion that pass through an internal channel of the metacarpophalangeal joint). 4, m. contrahentis digiti V. 5, m. contrahentis digiti V and $\mathrm{m}$. flexor minimus digiti V: common tendon of insertion on basal phalanx (the dashed line indicates the portion that pass through an internal channel of the metacarpophalangeal joint), 6, m. flexor minimus indicis. 7, m. flexor minimus indicis: tendon of insertion (the dashed line indicates the portion that pass through an internal channel of the metacarpophalangeal joint). 8, m. flexor minimus digiti III. 9, m. flexor minimus digiti III: tendon of insertion (the dashed line indicates the portion that pass through an internal channel of the metacarpophalangeal joint). 10, $\mathrm{m}$. flexor minimus digiti IV. 11, $\mathrm{m}$. flexor minimus digiti IV: tendon of insertion (the dashed line indicates the portion that pass through an internal channel of the metacarpophalangeal joint). 12, $\mathrm{m}$. flexor minimus digiti V. 13-15, mm. intermetacarpales I-III. 16-17, $\mathrm{m}$. interphalangeus digiti IV: medial and lateral slips,

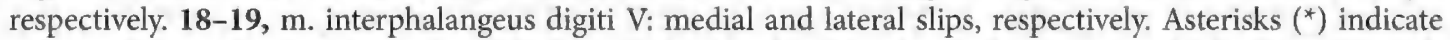
unremoved muscles from more superficial muscle layers other than the one being exposed. Characters figured: c8.1, presence of $\mathrm{m}$. flexor minimus indicis (pl. $8 \mathrm{C}$ ). $\mathrm{c} 9.0$, $\mathrm{m}$. flexor minimus digiti IV ventral to the $\mathrm{m}$. intermetacarpalis II (pl. 8C). $99.2, \mathrm{~m}$. flexor minimus digiti IV ventral and dorsal to the $\mathrm{m}$. intermetacarpalis II (pl. 8B). Scale bars $=0.5 \mathrm{~mm}$. 

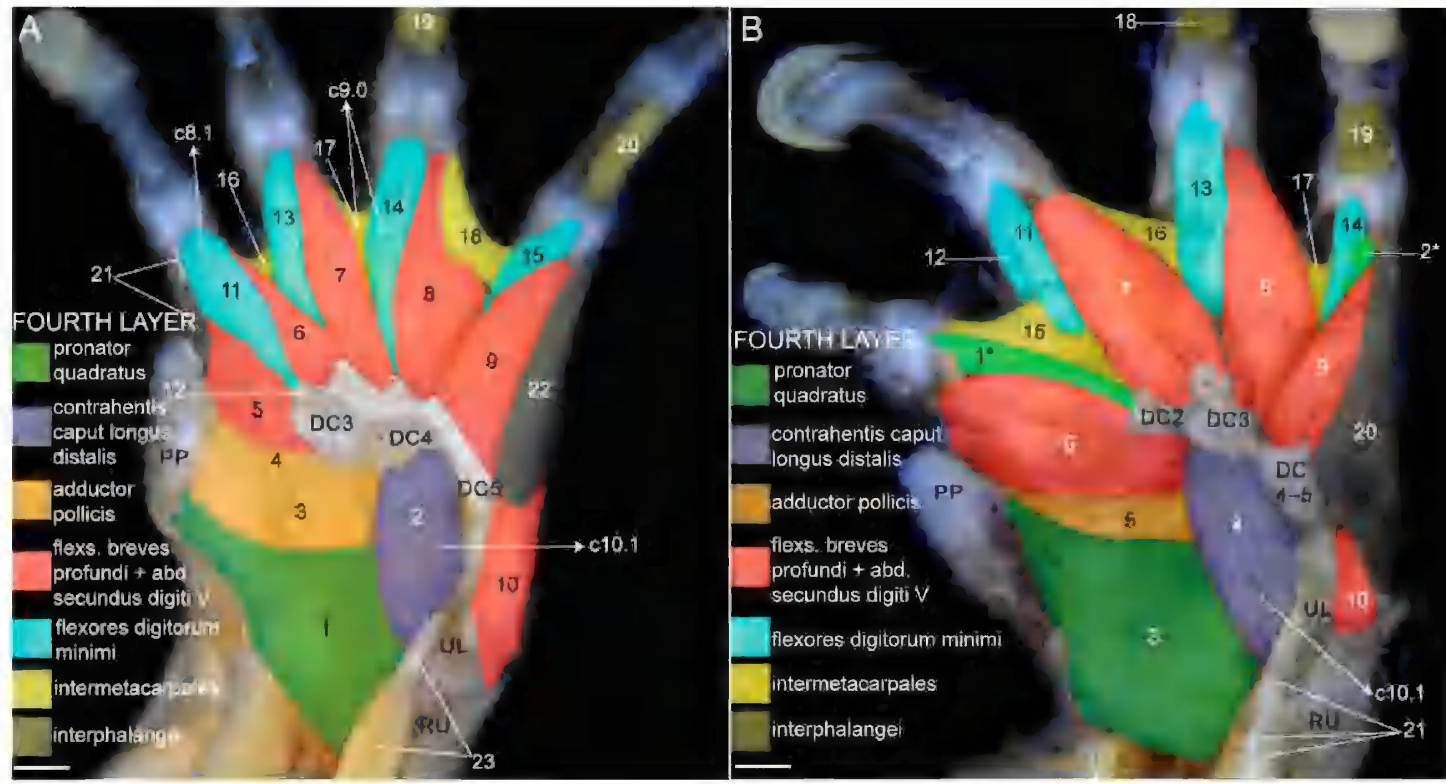

PLATE 9. Palmar surface showing the fourth layer of muscles. A. Right hand (reflected for comparison with other plates) of Ascaphus truei (KU 153228). Elements figured: 1, m. pronator quadratus. 2, m. contrahentis caput longus distalis. 3, m. adductor pollicis: portion from distal carpals 4 and 5. 4, m. adductor pollicis: portion from distal carpal 3.5, medial $\mathrm{m}$. flexor indicis brevis profundus. 6, lateral $\mathrm{m}$. flexor indicis brevis profundus. 7-9, mm. flexores breves profundi digitorum III-V. 10, m. abductor secundus digiti V. 11, m. flexor minimus indicis (probably fused with the $\mathrm{m}$. contrahentis indicis). 12, tendon of origin from distal carpal 3 that might correspond to the $\mathrm{m}$. contrahentis indicis, which is probably present and fused with the $\mathrm{m}$. flexor minimus indicis. 13-15, $\mathrm{mm}$. flexores minimi digitorum III-V. 16-18, $\mathrm{mm}$. intermetacarpales I-III. 19-20, mm. interphalangei digitorum IV-V: medial and lateral slips fused into a single muscular body. 21, unidentified muscle with origin on the prepollex, and insertion on metacarpal II and on metacarpophalangeal joint. 22, unidentified muscle that probably corresponds to the lateral $\mathrm{m}$. lumbricalis brevis digiti $\mathrm{V}$, the $\mathrm{m}$. abductor digiti minim, or a fusion of both muscles. $23, \mathrm{~m}$. contrahentium caput longus proximalis: fleshy portion (delimited by a dark line) and tendon of insertion (shaded and labeled for discussion; this forearm muscle is not part of the muscles studied in this work). B. Left hand of Bombina orientalis (KU 38654). Elements figured: $\mathbf{1}^{*}, \mathrm{~m}$. contrahentis indicis. $\mathbf{2}^{*}, \mathrm{~m}$. contrahentis digiti V: distal unremoved portion corresponding to the lateral insertion on metacarpal $\mathrm{V}$ (laterally to the $\mathrm{m}$. flexor minimus digiti $\mathrm{V}$; see pl. 8A). 3, m. pronator quadratus, $4, \mathrm{~m}$. contrahentis caput longus distalis, 5, m. adductor pollicis (origin on distal carpal 4-5, hidden by the $\mathrm{m}$. contrahentis caput longus distalis). 6, medial $\mathrm{m}$. flexor indicis brevis profundus. 7-9, $\mathrm{mm}$. flexores breves profundi digitorum III-V. 10, m. abductor secundus digiti V. 11, m. flexor minimus digiti III: ventral origin/head with respect to the $\mathrm{m}$. intermetacarpalis $1.12, \mathrm{~m}$. flexor minimus digiti III: dorsal origin/head with respect to the $\mathrm{m}$. intermetacarpalis I. 13, m. flexor minimus digiti IV: ventral origin/head with respect to the $\mathrm{m}$. intermetacarpalis II (the dorsal head is hidden by it), $\mathbf{1 4}, \mathrm{m}$, flexor minimus digiti $\mathrm{V}$. 15-17, $\mathrm{mm}$. intermetacarpales I-III. 18-19, $\mathrm{mm}$. interphalangei digitorum IV-V: medial and lateral slips fused into a single muscular body. 20, muscle from the flexor plate and insertion on the lateral surface of metacarpal $\mathrm{V}$ that may correspond to the $\mathrm{m}$. abductor digiti minimi. 21, $\mathrm{m}$. contrahentium caput longus proximalis: fleshy portion (delimited by a dark line) and tendon of insertion (shaded and labeled for discussion; this forearm muscle is not part of the muscles studied in this work). 

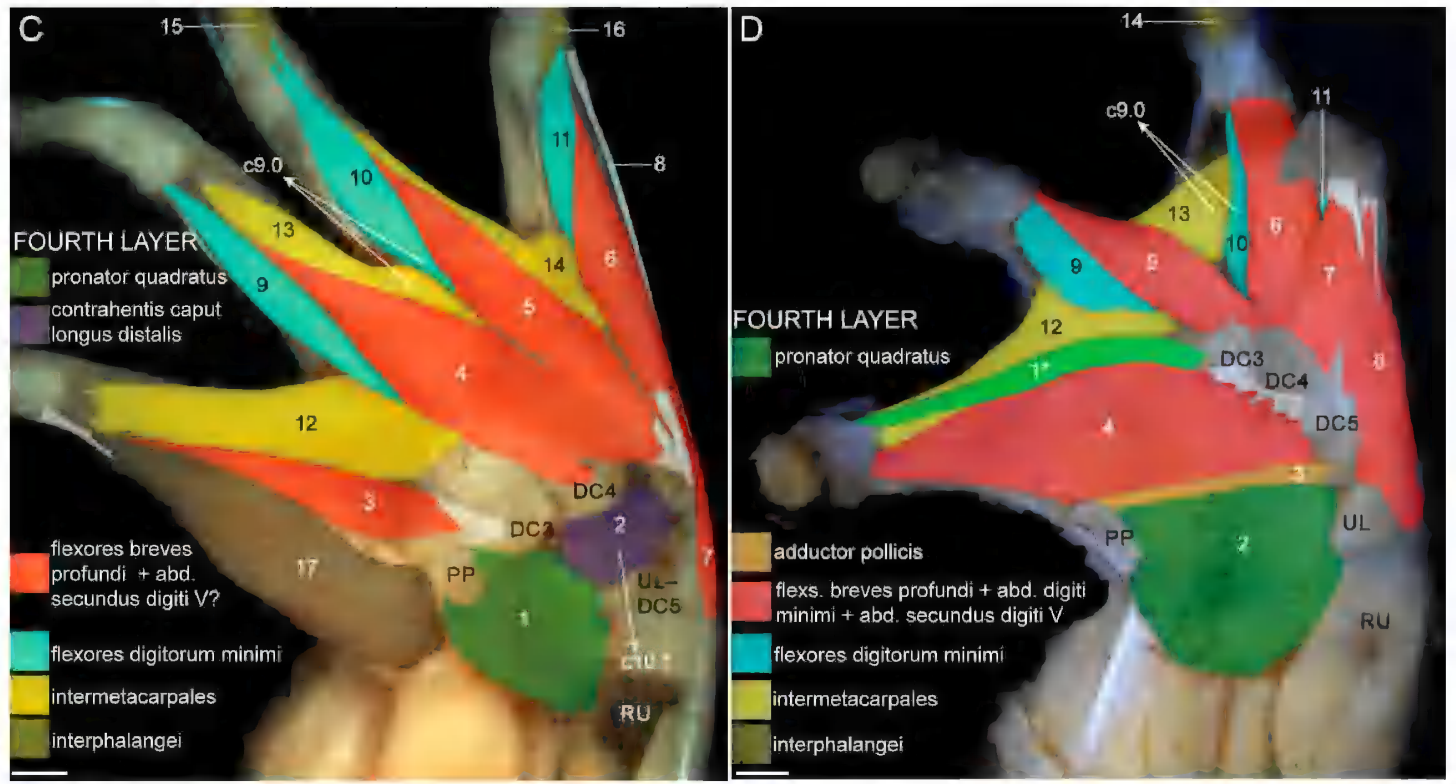

PLATE 9. (continued) C. Right hand (reflected for comparison with other plates) of Pipa carvalhoi (MZUSP 81992). Elements figured: 1, m. pronator quadratus. 2, m. contrahentis caput longus distalis. 3, medial m. flexor indicis brevis profundus. 4-6, mm. flexores breves profundi digitorum III-V. 7-8, an abductor muscle (part of the $\mathrm{m}$. abductor secundus digiti $\mathrm{V}$ ?) with long tendon of insertion on the lateral surface of the metacarpophalangeal joint (a typical $\mathrm{m}$. abductor secundus digiti $\mathrm{V}$ is also present, but it is deeper and hidden by it). 9-11, mm. flexores minimi digitorum III-V. 12-14, mm. intermetacarpales I-III. 15-16, mm. interphalangei digitorum IV-V: intermediate slips. 17, unidentified abductor muscle of digit II. D. Right hand (reflected for comparison with other plates) of Scaphiopus hurterii (KU 187903). Note the absence of the $\mathrm{m}$. contrahentis caput longus distalis. Elements figured: $\mathbf{1}^{*}, \mathrm{~m}$. contrahentis indicis. 2, m. pronator quadratus. 3, $\mathrm{m}$. adductor pollicis. $\mathbf{4}$, medial $\mathrm{m}$. flexor indicis brevis profundus. $\mathbf{5 - 7}, \mathrm{mm}$. flexores breves profundi digitorum III-V. 8, fused $\mathrm{m}$. abductor digiti minimi and $\mathrm{m}$. abductor secundus digiti V. 9-11, $\mathrm{mm}$. flexores minimi digitorum III-V. 12-13, mm. intermetacarpales I-II. 14, m. interphalangeus digiti IV: intermediate slip. Asterisks $\left.{ }^{*}\right)$ indicate unremoved muscles from the third layer. Some tendons of origin and insertion are not shaded. Characters figured: $\mathrm{c} 8.1$, presence of $\mathrm{m}$. flexor minimus indicis (pl. 9A). c9.0, m. flexor minimus digiti IV ventral to the $\mathrm{m}$. intermetacarpalis II (pl. 9A, C-D). c10.1, presence of $\mathrm{m}$. contrahentis caput longus distalis (pl. 9A-C). Scale bars $=0.5 \mathrm{~mm}$. 


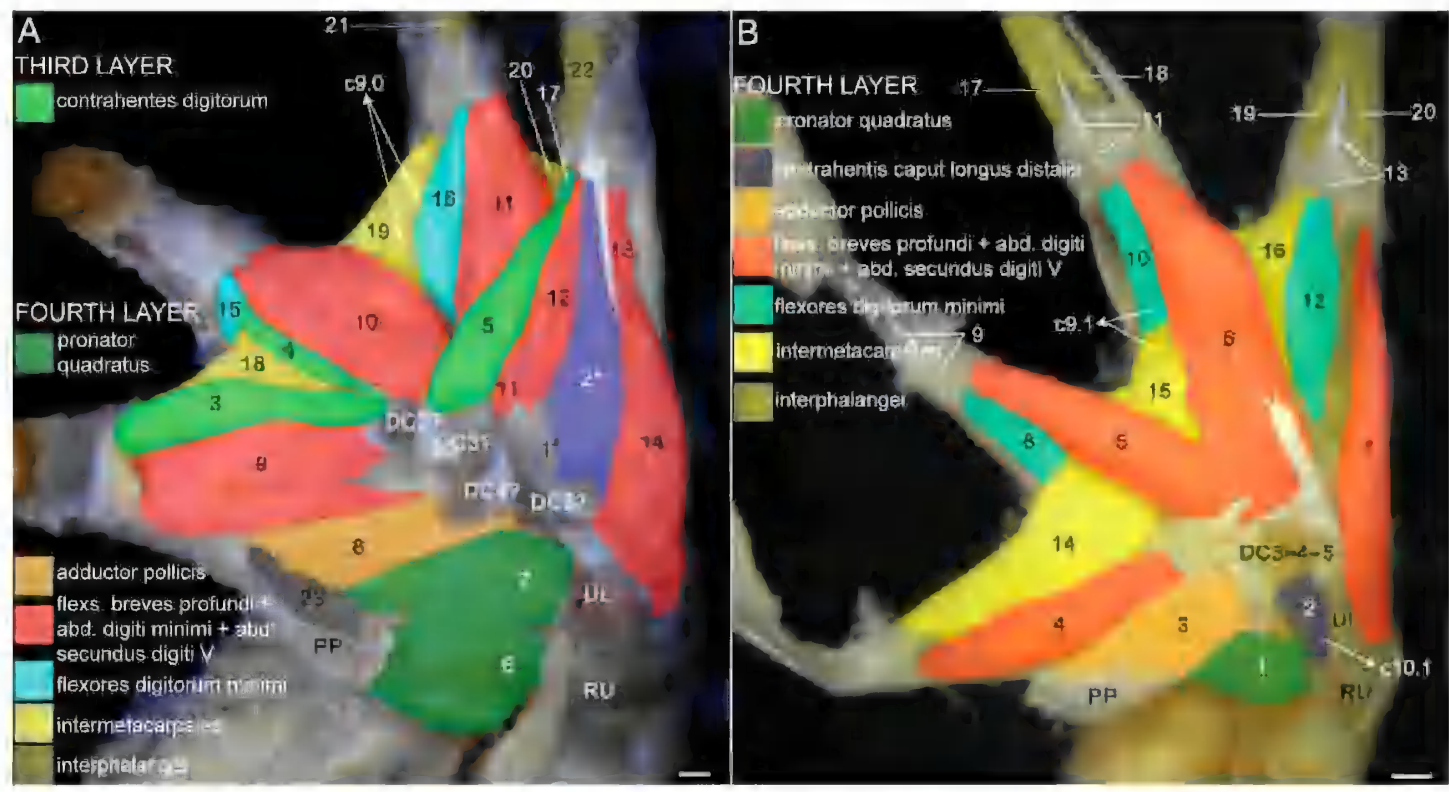

PLATE 10. A. Palmar surface showing the third layer of the left hand of Scutiger mammatus (FMNH 282284). Muscles of the fourth (deepest) layer are also colored for discussion. Some tendons of origin and insertion are not shaded. Specific identities of the distal carpals are labeled with question mark since the precise carpal morphology (i.e., fusion or independence of these distal carpals) requires corroboration. Identities and limits among the muscles of digit $V$ (with the exception of the $\mathrm{m}$, flexor minimus and $\mathrm{m}$. interphalangeus) are tentative because they are partially fused in their origins or insertions. Note the absence of the $\mathrm{m}$. contrahentis caput longus distalis. Elements figured: $\mathbf{1}^{*}$, unremoved laterodistal portion of the flexor plate from where the putative lateral $\mathrm{m}$. lumbricalis brevis digiti $\mathrm{V}$ originates. $2^{*}$, lateral $\mathrm{m}$. lumbricalis brevis digiti $\mathrm{V}$ (tentative identification and color). 3, m. contrahentis indicis. 4-5, mm. contrahentes digitorum III and V. 6-7, $\mathrm{m}$. pronator quadratus: head from the radioulna and head from the ulnare, respectively $8, \mathrm{~m}$. adductor pollicis. 9 , medial $\mathrm{m}$. flexor indicis brevis profundus. 10-12, $\mathrm{mm}$. flexores breves profundi digitorum III-V. 13, portion of the abductors of digit $\mathrm{V}$ that probably corresponds to the $\mathrm{m}$. abductor digiti minimi. 14, portion of the abductors of digit $\mathrm{V}$ that probably corresponds to a fused $\mathrm{m}$. abductor digiti minimi and $\mathrm{m}$. abductor secundus digiti V. 15-17, mm. flexores minimi digitorum III-V. 18-20, mm. intermetacarpales I-III. 21-22, mm. interphalangei digitorum IV-V: intermediate slips. 23, unremoved distal portion of an unidentified muscle with origin from the flexor plate and insertion on the prepollex (see pl. 7). B. Palmar surface showing the fourth layer of muscles of the right hand (reflected for comparison with other plates) of Eleutherodactylus coqui (KU 180490). Some tendons of origin and insertion are not shaded. The $\mathrm{m}$. flexor brevis profundus digiti $V$ was removed. Elements figured: 1, m. pronator quadratus. 2, m. contrahentis caput longus distalis. 3, $\mathrm{m}$. adductor pollicis. 4, medial $\mathrm{m}$. flexor indicis brevis profundus. $5-6, \mathrm{~mm}$. flexores breves profundi digitorum III-IV. 7, fused $\mathrm{m}$. abductor digiti minimi and $\mathrm{m}$. abductor secundus digiti V. 8, m. flexor minimus digiti III. 9, $\mathrm{m}$. flexor minimus digiti III: tendon of insertion (the dashed line indicates the portion that pass through an internal channel of the metacarpophalangeal joint), 10, $\mathrm{m}$. flexor minimus digiti IV. 11, $\mathrm{m}$. flexor minimus digiti IV: tendon of insertion (the dashed line indicates the portion that pass through an internal channel of the metacarpophalangeal joint). 12, m. flexor minimus digiti V. 13, m. flexor minimus digiti V: tendon of insertion (the dashed line indicates the portion that pass through an internal channel of the metacarpophalangeal joint). 14-16, mm. intermetacarpales I-III. 17-18, m. interphalangeus digiti IV: medial and lateral slip, respectively. 19-20, m. interphalangeus digiti V: medial and lateral slip, respectively. Asterisks (*) indicate unremoved muscles from second layer. Characters figured: $c 9.0, \mathrm{~m}$. flexor minimus digiti IV ventral to the $\mathrm{m}$. intermetacarpalis II (pl. 10A). c9.1, m. flexor minimus digiti IV dorsal to the m. intermetacarpalis II (pl. 10B). c10.1, presence of $\mathrm{m}$. contrahentis caput longus distalis (pl. 10B). Scale bars $=0.5 \mathrm{~mm}$. 


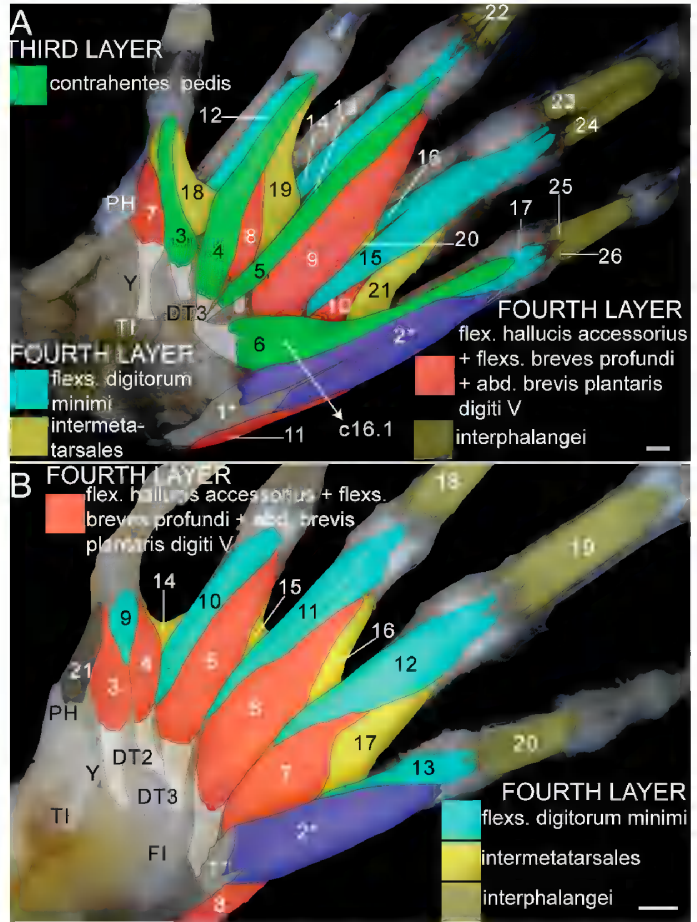

PLATE 11. A. Plantar surface showing the third layer of muscles of the left foot of Bombina orientalis (KU 38654). Muscles of the fourth layer are also colored for discussion. Note the presence of the m. contrahentis pedis digiti $\mathrm{V}$ (muscle number 6). Elements figured: $1^{*}$, unremoved distal portion of the aponeurosis plantaris that serves of origin of the lateral $\mathrm{m}$. lumbricalis brevis digiti V. $2^{*}$, lateral m. lumbricalis brevis digiti V. 3, m. contrahentis pedis hallucis. 4-6, $\mathrm{mm}$. contrahentes pedis digitorum II-III and V. 7, $\mathrm{m}$. flexor hallucis accessorius. 8-10, $\mathrm{mm}$. flexores breves profundi digitorum II-IV. 11, putative $\mathrm{m}$. abductor brevis plantaris digiti V. 12, m. flexor minimus digiti II. 13, m. flexor minimus digiti III: ventral origin/head with respect to the $\mathrm{m}$. intermetatarsalis II. 14, m. flexor minimus digiti III: dorsal origin/ head with respect to the $\mathrm{m}$. intermetatarsalis II. 15, $\mathrm{m}$. flexor minimus digiti IV: ventral origin/head with respect to the $\mathrm{m}$. intermetatarsalis III. 16, $\mathrm{m}$. flexor minimus digiti IV: dorsal origin/head with respect to the $\mathrm{m}$. intermetatarsalis III. 17, $\mathrm{m}$. flexor minimus digiti V. 18-21, mm. intermetatarsales I-IV. 22, m. interphalangeus digiti III: fused medial and lateral slips. 23-24, m. interphalangeus proximalis digiti IV: medial and lateral slips, respectively. 25-26, $\mathrm{m}$. interphalangeus proximalis digiti V: medial and lateral slips, respectively. B. Plantar surface showing the fourth layer of muscles of the left foot of Ascaphus truei (KU 153228). Note the presence of the $\mathrm{m}$. flexor hallucis brevis profundus (muscle number 4). Elements figured: $\mathbf{1}^{*}$, unremoved distal portion of the aponeurosis plantaris that serves of origin of the lateral $\mathrm{m}$. lumbricalis brevis digiti V. $2^{*}$, lateral $\mathrm{m}$. lumbricalis brevis digiti V. 3, m. flexor hallucis accessorius. 4, m. flexor hallucis brevis profundus. 5-7, mm. flexores breves profundi digitorum II-IV. 8, m. abductor brevis plantaris digiti V. 9, m. flexor minimus hallucis. 10-13, $\mathrm{mm}$. flexores minimi digitorum II-V. 14-17, mm. intermetatarsales I-IV. 18, $\mathrm{m}$. interphalangeus digiti III: fused medial and lateral slips. 19, m. interphalangeus proximalis digiti IV: fused medial and lateral slips. 20, m. interphalangeus proximalis digiti $\mathrm{V}$ : fused medial and lateral slips. 21, unidentified muscle with origin on the prehallux and insertion on metatarsal I. The distal fleshy portions and tendons of insertion of the $\mathrm{mm}$. contrahentes pedis and $\mathrm{mm}$. flexores digitorum minimi pass through an internal channel of the metatarsophalangeal joint of their respective digit; since the connective tissue that forms these channels are thin and translucent, we shaded these portions without discontinuities (see for example pl. 4C-D, shaded indicating its apparent discontinuity as the tendons pass through the channel). Asterisks $\left(^{*}\right)$ indicate unremoved muscles from the second layer. Characters figured: $c 16.1$, presence of $\mathrm{m}$. contrahentis pedis digiti V (pl. 11A). Scale bars $=0.5 \mathrm{~mm}$. 

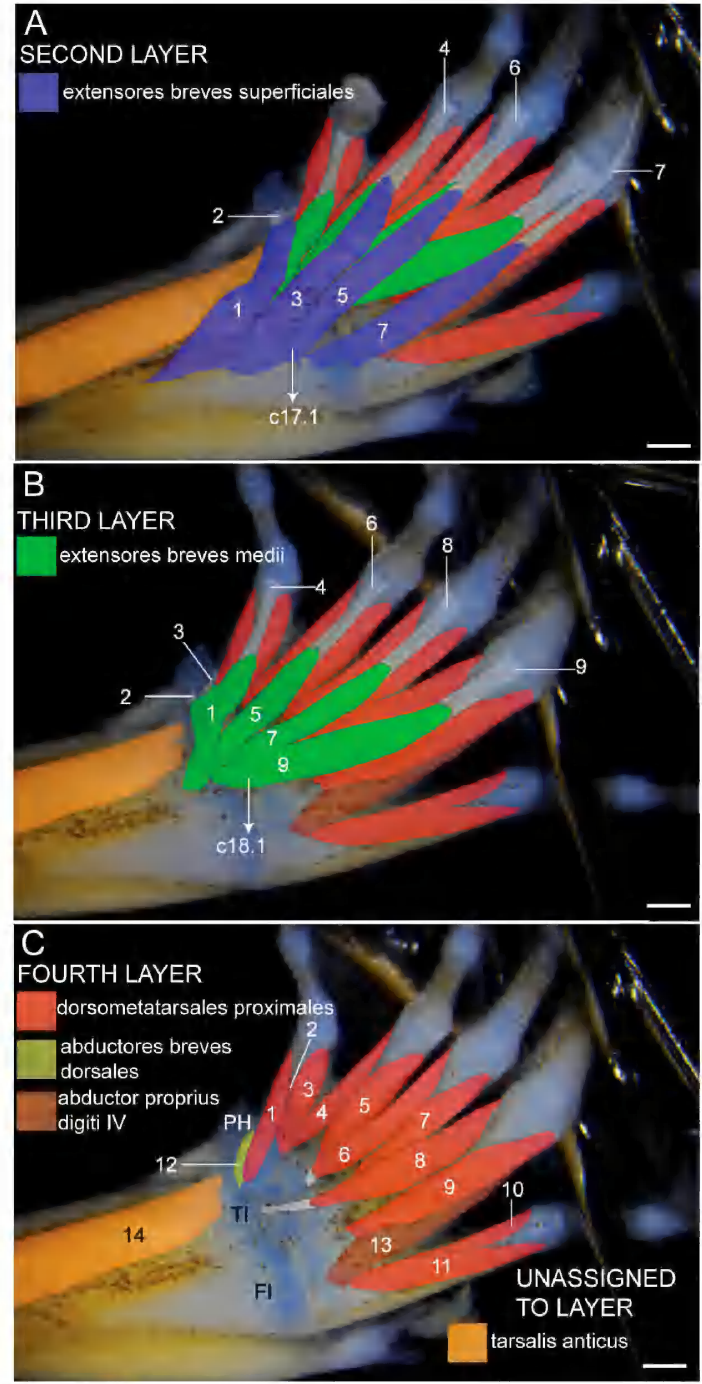

PLATE 12. Dorsal surface of the left foot (reflected for comparison with other plates) of Alytes obstetricans ( $C M$ 60824). Note the presence of the $m$. extensor brevis medius digiti IV. The shading of the tendons of insertion of the $\mathrm{mm}$. extensores breves superficiales and medii are approximate, and those of the $\mathrm{mm}$. dorsometatarsales proximales are not shaded. A. Second layer of muscles (mm. extensores breves superficiales). Elements figured: 1-2, $\mathrm{m}$. extensor brevis superficialis hallucis: fleshy portion and tendon of insertion on prehallux. $3, \mathrm{~m}$. extensor brevis superficialis digiti II. 4, m. extensor brevis superficialis digiti II: common tendon of insertion with $\mathrm{m}$. extensor brevis medius digiti II.
5, m. extensor brevis superficialis digiti III. 6, m. extensor brevis superficialis digiti III: common tendon of insertion with $\mathrm{m}$. extensor brevis medius digiti III. 7, m. extensor brevis superficialis digiti IV. B. Third layer of muscles ( $\mathrm{mm}$. extensores breves medii). Elements figured: 1, m. extensor brevis medius hallucis. 2-4, m. extensor brevis medius hallucis: tendon of insertion on prehallux, metatarsal I, and metatarsophalangeal joint, respectively. 5, m. extensor brevis medius digiti II. 6, m. extensor brevis medius digiti II: common tendon of insertion with $\mathrm{m}$. extensor brevis superficialis digiti II. 7, m. extensor brevis medius digiti III. 8, m. extensor brevis medius digiti III: common tendon of insertion with $\mathrm{m}$. extensor brevis superficialis digiti III. 9, m. extensor brevis medius digiti IV. C. Fourth layer of muscles (mm. dorsometatarsales, $\mathrm{mm}$. abductores breves dorsales, and $\mathrm{m}$. abductor proprius digiti IV); the $\mathrm{m}$. tarsalis anticus is also shown. Elements figured: 1, medial $\mathrm{m}$. dorsometatarsalis hallucis proximalis: slip from element Y. 2, medial $\mathrm{m}$. dorsometatarsalis hallucis proximalis: slip from metatarsal I. 3, lateral m. dorsometatarsalis hallucis proximalis. 4 , medial $\mathrm{m}$. dorsometatarsalis proximalis digiti II. 5, lateral $\mathrm{m}$. dorsometatarsalis proximalis digiti II. 6, medial m. dorsometatarsalis proximalis digiti III. 7, lateral $\mathrm{m}$. dorsometatarsalis proximalis digiti III. 8, medial $\mathrm{m}$. dorsometatarsalis proximalis digiti IV. 9, lateral $\mathrm{m}$. dorsometatarsalis proximalis digiti IV. 10, medial $\mathrm{m}$. dorsometatarsalis proximalis digiti V. 11, lateral $\mathrm{m}$. dorsometatarsalis proximalis digiti V. 12, m. abductor brevis dorsalis hallucis. 13, $\mathrm{m}$. abductor proprius digiti IV. 14, m. tarsalis anticus. Characters figured: $\mathrm{cl}$.1, presence of $\mathrm{m}$. extensor brevis superficialis digiti III (pl. 12A). c18.1, presence of $\mathrm{m}$. extensor brevis medius digiti IV (pl. 12B). Scale bars $=0.5 \mathrm{~mm}$. 


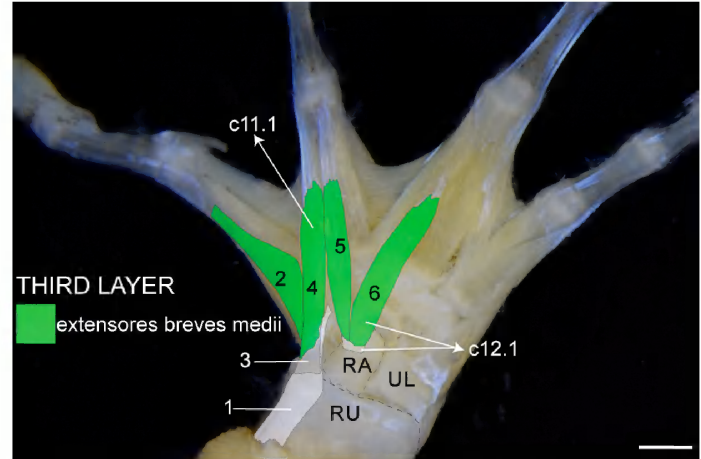

PLATE 13. Dorsal surface showing the third layer of muscles of the right hand of Megophrys aceras (KU 327892). Note the presence of the slip of the $\mathrm{m}$. extensor brevis medius digiti III from the radioulna, originating on the tendon of insertion of the $\mathrm{m}$. extensor carpi radialis. Tendons of insertion of the hand muscles not shaded. Elements figured: 1, m. extensor carpi radialis: medial portion of the tendon of insertion. 2, m. extensor indicis brevis medius. 3, $\mathrm{m}$. extensor brevis medius digiti III: slip from radioulna: tendon of origin. $4, \mathrm{~m}$. extensor brevis medius digiti III: slip from radioulna (the slip of the $\mathrm{m}$. extensor brevis medius digiti III from the element $Y$ is completely hidden by it). $\mathbf{5}, \mathrm{m}$. extensor brevis medius digiti III: slip from radiale. 6, m. extensor brevis medius digiti IV. Characters figured: c11.1, presence of the slip originating from the radioulna of the $\mathrm{m}$. extensor brevis medius digiti III. c12.1, presence of the origin from the radiale of the $\mathrm{m}$. extensor brevis medius digiti IV. Scale bar $=1 \mathrm{~mm}$.

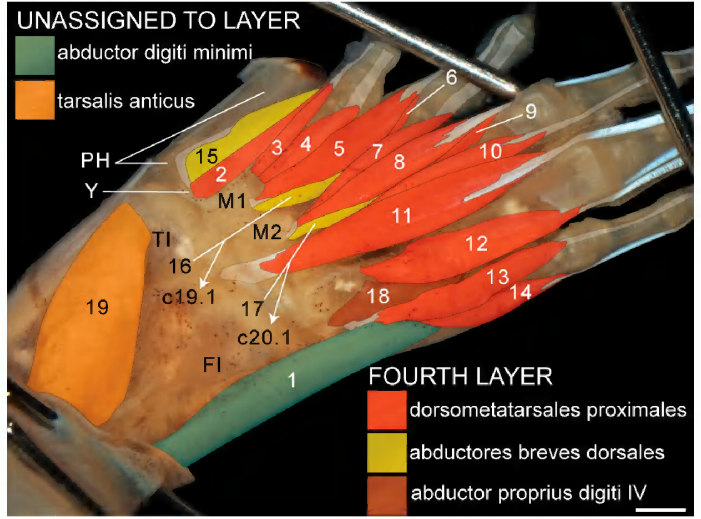

PLATE 14. Dorsal surface showing the fourth layer of muscles of the right foot of Neobatrachus aquilonius (KU 93576); the m. abductor digiti minimi and the $\mathrm{m}$. tarsalis anticus are also shown. Note the presence of $\mathrm{mm}$. abductores breves dorsales in digits II and III. The shading of the tendons of insertion of the $\mathrm{mm}$. dorsometatarsales proximales is approximate. Elements figured: 1, m. abductor digiti minimi. 2, medial $\mathrm{m}$. dorsometatarsalis hallucis proximalis: slip from element Y. 3, medial m. dorsometatarsalis hallucis proximalis: slip from metatarsal I. 4, lateral m. dorsometatarsalis hallucis proximalis. 5, medial $\mathrm{m}$. dorsometatarsalis proximalis digiti II: slip from metatarsal I. 6, medial m. dorsometatarsalis proximalis digiti II: slip from metatarsal II. 7, lateral m. dorsometatarsalis proximalis digiti II. 8, medial m. dorsometatarsalis proximalis digiti III: slip from metatarsal II. 9, medial $\mathrm{m}$. dorsometatarsalis proximalis digiti III: slip from metatarsal III. 10, lateral $\mathrm{m}$. dorsometatarsalis proximalis digiti III. 11, medial m. dorsometatarsalis proximalis digiti IV. 12, lateral $\mathrm{m}$. dorsometatarsalis proximalis digiti IV. 13, medial $\mathrm{m}$. dorsometatarsalis proximalis digiti V. 14, lateral $\mathrm{m}$. dorsometatarsalis proximalis digiti V. 15, m. abductor brevis dorsalis hallucis. 16-17, $\mathrm{mm}$. abductores breves dorsales digitorum II-III, respectively. 18, m. abductor proprius digiti IV. 19, tarsalis anticus. Characters figured: $\mathrm{c} 19.1$, presence of $\mathrm{m}$. abductor brevis dorsalis digiti II. c20.1, presence of $\mathrm{m}$. abductor brevis dorsalis digiti III. Scale bar $=1 \mathrm{~mm}$. 



\title{
Scientific Publications of the American Museum of Natural History
}

AMERICAN MUSEUM NOVITATES

BULletin of the AmERICAN MUSEUM of Natural History

Anthropological Papers of the american Museum of Natural history

\author{
Publications Committee \\ ROBERT S. VOSS, CHAIR \\ BOARD OF EDITORS \\ Jin Meng, Paleontology \\ LORENZO PRENDINI, INVERTEBRATE ZOOLOGY \\ ROBERT S. VOSS, VerTEBRATE ZOOLOGY \\ Peter M. Whiteley, Anthropology \\ MANAGING EDITOR \\ MARY KNIGHT
}

Submission procedures can be found at http://research.amnh.org/scipubs

All issues of Novitates and Bulletin are available on the web (http://digitallibrary.amnh. org/dspace). Order printed copies on the web from:

http://shop.amnh.org/a701/shop-by-category/books/scientific-publications.html

or via standard mail from:

American Museum of Natural History-Scientific Publications

Central Park West at 79th Street

New York, NY 10024

(2) This paper meets the requirements of ANSI/NISO Z39.48-1992 (permanence of paper).

ON THE COVER: ARBOREAL FROGS USUALLY HAVE MORE GROUPS OF HAND AND FOOT MUSCLES AND GREATER COMPLEXITY THAN OTHER SPECIES. AS SUCH, THE ARBOREAL HYLID TRIPRION PETASATUS SERVES AS OUR MODEL TO DEFINE THE FOUR LAYERS IN WHICH THE MUSCLES ARE ARRANGED (EACH HAND IN THE BACKGROUND), IDENTIFY MUSCLE GROUPS (COLORS), AND MUSCLES (NUMBERS) AND ATTRIBUTE PRESUMED FUNCTIONS. PHOTO OF LIVE FROG BY TARAN GRANT. BACKGROUND IMAGES BY BORIS BLOTTO. 